University of Tennessee Health Science Center

UTHSC Digital Commons

\title{
Discovery of Natural Product-based Antimycobacterial Agents Effective against Non-replicating Bacilli
}

Shajila Siricilla

University of Tennessee Health Science Center

Follow this and additional works at: https://dc.uthsc.edu/dissertations

Part of the Medicinal and Pharmaceutical Chemistry Commons, Natural Products Chemistry and Pharmacognosy Commons, and the Pharmaceutics and Drug Design Commons

\section{Recommended Citation}

Siricilla, Shajila (http://orcid.org/0000-0003-3243-2812), "Discovery of Natural Product-based Antimycobacterial Agents Effective against Non-replicating Bacilli" (2017). Theses and Dissertations (ETD). Paper 434. http://dx.doi.org/10.21007/etd.cghs.2017.0563. 


\title{
Discovery of Natural Product-based Antimycobacterial Agents Effective against Non-replicating Bacilli
}

\author{
Abstract \\ New antimycobacterial molecules that kill non-replicating Mycobacterium tuberculosis (Mtb) were \\ identified by screening libraries of synthetic natural products. De novo screening of a 400 -membered \\ library of aurachin RE analogs resulted in discovery of UT-317 ((R)-20). UT-317 is a selective vitamin K2 \\ biosynthesis (MenA) inhibitor that killed replicating and non-replicating Mtb at $2.31 \mu \mathrm{g} / \mathrm{mL}$ (MIC) and 0.85 \\ $\mu \mathrm{g} / \mathrm{mL}$, respectively. A 50-membered library of capuramycin analogs was evaluated in their enzymatic \\ inhibitory activities against translocase I (MraY/MurX) and prenyl-phosphate-GlcNAc-1-phosphate \\ transferase (WecA). UT-01320 (45) is identified as a selective WecA inhibitor that kills both replicating and \\ non-replicating Mtb at $1.50 \mu \mathrm{g} / \mathrm{mL}$ (MIC) and $2.58 \mu \mathrm{g} / \mathrm{mL}$, respectively. UT-01320 killed the intracellular \\ Mtb much faster than the first-line TB drugs such as isoniazid and rifampicin. A strong antimycobacterial \\ agent, UT-800 (64) was identified by screening of a 50-membered library of pleuromutilin derivatives. \\ UT-800 is a protein biosynthesis (50s ribosome) inhibitor which has activity focused against Mtb. UT-800 \\ killed replicating and non-replicating Mtb at $0.83 \mu \mathrm{g} / \mathrm{mL}$ (MIC) and $1.20 \mu \mathrm{g} / \mathrm{mL}$, respectively. In the course \\ of these works, fluorescent probes, Park's nucleotide-Nع-C6-FITC (32, for MraY/MurX) and UDP- \\ glucosamine-C6-FITC (46, for WecA) were developed. These fluorescent probes enable us to screen the \\ polyprenyl-phosphate $\mathrm{N}$-acetylhexosamine 1-phosphate transferase enzyme superfamily (e.g. MraY/ \\ MurX, WecA, AlgH, and DPAGT1) in high-throughput manner.

\section{Document Type \\ Dissertation} \\ Degree Name \\ Doctor of Philosophy (PhD) \\ Program \\ Pharmaceutical Sciences

\section{Research Advisor} \\ Michio Kurosu, Ph.D.

\section{Keywords} \\ MenA inhibitors, Mycobacterium tuberculosis, mycolyl arabinogalactan, Phosphotransferase inhibitors, \\ Pleuromutilin analogs, WecA and MurX inhibitors

\section{Subject Categories} \\ Medicinal and Pharmaceutical Chemistry | Medicine and Health Sciences | Natural Products Chemistry \\ and Pharmacognosy | Pharmaceutics and Drug Design | Pharmacy and Pharmaceutical Sciences
}


Discovery of Natural Product-based Antimycobacterial Agents Effective against Non-replicating Bacilli

\author{
A Dissertation \\ Presented for \\ The Graduate Studies Council \\ The University of Tennessee \\ Health Science Center \\ In Partial Fulfillment \\ Of the Requirements for the Degree \\ Doctor of Philosophy \\ From The University of Tennessee
}

By

Shajila Siricilla

May 2017 
Chapter 2 (c) 2012 by American Chemical Society.

Portions of Chapter 3 (C) 2014 by Nature.

Portions of Chapter 3 (C) 2015 by ScienceDirect.

Portions of Chapter 3 (C) 2016 by ScienceDirect.

All other material (c) 2017 by Shajila Siricilla.

All rights reserved. 


\section{DEDICATION}

To my husband Manoj Siricilla for his constant support, saneness, and for not letting me lose focus on the big picture of life.

To my parents Ummuselma Thacha Paramban and Abid Poolakkal for their endless love and for teaching me to dream.

To my daughter Zoe Siricilla who kept me rejuvenated with all her liveliness. 


\section{ACKNOWLEDGEMENTS}

My journey that led me on the path of a Doctorate would not have been possible without the overwhelming support and love from everyone that have crossed my path in the last six years. I express my heartfelt gratitude to all these people who helped me realize my dream.

First and foremost, I would like to express my deepest gratitude to my primary advisor, Dr. Michio Kurosu. He has been incredibly patient with me over the years and provided me numerous opportunities to inculcate essential habits and attributes that helped me transform into a real scientist. I sincerely thank him for inspiring me to strive for perfection. He certainly has been the driving force in my academic life for the last six years. I immensely appreciate the countless number of hours he spent teaching me research and guiding me through various projects. It is my pleasure to be a part of his team. Most importantly I thank him for not giving up on me and encouraging me to go the extra mile.

I am extremely fortunate to have a very supportive dissertation committee. I thank Dr. Duane Miller, Dr. Richard Lee, Dr. Bernd Meibohm and Dr. Scott Franzblau for taking time from their busy schedules to advise me on my projects. Dr. Miller has always been there for me. His friendly smile always brought back hope when everything seemed the most difficult. I greatly appreciate him agreeing to continue to serve in my committee even post retirement. I thank Dr. Lee for providing me critics and comments on my work and presentations. I thank Dr. Meibohm for the support through out the process and the opportunity to discuss with his team on several pharmacokinetic principles and procedures. It was a pleasure to be in his pharmacokinetics classes and I am fortunate to be his student. I am especially greatful to Dr. Franzblau and his team for generating important data through LORA assays which has been significant for my dissertation. I am extremely greatful for the associations of these great scientists because they made my scientific career.

No words can express the value of a good lab mate and no words I write can convey my deep held appreciation for the post-doctoral researcher of our lab, Dr. Katsuhiko Mitachi. He is a spectacular chemist with a great work ethic. He provided me with the MurX, WecA substrates, inhibitor molecules and several other compounds that helped me progress my research on various projects. His unconditional support has helped me complete many projects on time. I thank Dr. Mitachi for his undying spirit and extending his support every time when I needed it. I would like to thank Dr. Joy Debnath, for helping me kick start the graduate college in the first semester. He trained and mentored me despite his busy schedule. Dr. Debnath was instrumental in synthesizing all MenA inhibitors. He assisted me with several assays for the menaquinone project that kick started my research life at University of Tennessee. I thank Dr. Bilal Aleiwi for being a wonderful lab mate and keeping a friendly face amidst the inherent chaos of scientific research. I want to thank Yong for synthesizing many molecules for my screening projects. 
I thank Dr. William Clemons and his group for all the fruitful collaborations with the MraY project. Their effort in obtaining purified MraY enzyme has helped me obtain significant data for my project. I thank Dr. Ying Kong and his team for being a great help in obtaining intra macrophage data for our novel compounds. I thank Dr. Yinduo Ji for his help with sequencing studies. I am grateful to Dr. Frank Park and Jeff Presley for doing in vivo toxicity studies and allowing me to use the fluorescent microscope. I would like to thank Ewa Swiezewska for sending us prenyl phosphates. None of my work would have been completed without the help of many scientists who were willing to help me without expecting anything in return. I thank Dr. Michael McNeil for taking time to answer my questions about WecA enzyme assays in the middle of his retirement life. I appreciate Anna Grzegorzewicz for taking time to send me the E. coli strain that overexpressed WecA. That bacterial strain was a turning point in completing one of the major projects for my dissertation.

The team at RBL has been terrific and I cannot thank them enough for their support over the years. I am especially greatful to Dr. Jan Peters, Jennifer Stabenow and Robert Benson for their timely response to our requests every single time. The RBL team played a very significant role in keeping me sane throughout the whole process. I would also like to thank Dr. John Cox from the Department of Molecular Biology for helping me get access to the ultracentrifuge when we did not have one and Dr. White for letting me use his rotors every time I needed it.

I would like to thank all the faculty of College of Pharmacy of UTHSC for their support, especially Dr. Isaac Donkor for giving me the opportunity to conduct Medicinal Chemistry recitations for the last five years. I am grateful for the trust he had on me with the class. Those large group sessions have helped me realize my teaching abilities. I would also like to thank Dr. Hassan Almoazen for his continuous support. I would like to acknowledge the administrative staff of College of Pharmacy and Graduate Health Sciences for their support. David Clark, Brenda Thornton, Corliss Finlay, Carol Taylor, Cynthia Crowe, Mary Newell, Sherri Jackson, Benita Williams, Felicia Martin, Connie Childs and express a special thanks to Shelley Cannioto for all the help, including the help following up with the administrative processes to ensure that I am assigned a post maternity room. I would like to thank the Department of Pharmaceutical Sciences and the University of Tennessee for providing me the opportunity and funding throughout my PhD program.

There are many friends and colleagues who deserve special thanks without whom I wouldn't have made this far in my academic life. I thank Dr. Pradeep Lukka and Santosh Wagh for all their time invested in helping me with microsomal stability and protein binding assays. I thank Pradeep for taking time to read through my dissertation and provide me with helpful suggestions. Diwa Koirala for lending an ear every time I felt the need to let off some steam and for teaching me electrophoresis. I thank Dr. Ashit Trivedi for being my go to guy for every question I had about science and life at UTHSC and for spreading a cheerful attitude. I also thank Dr. Dileep Janagam, Dr. Chetan Rathi, Dr. Jordan Toutounchian, Dr. Nivesh Mittal, Chandana Damera, Sabina Ranjit, Aarif 
Rahman, Josiah Ryman, Pallabita Chowdhury and Dr. Hemlata Patil for their humorous and wonderful company. I want to thank Shakiba Eslamimehr and Maddie Lemieux for being wonderful lab mates. I appreciate the many hours Maddie spent, proof reading my dissertation. I thank John Shelton for being a wonderful friend and keeping my spirits high everytime, and for being a link to the outside world. I also thank all other friends who helped me keep my sanity and my life in Memphis very memorable.

There is a special feeling of gratitude for my amazing husband, Manoj. Without him, nothing would have been possible. His constant motivation and support kept me going. I would like to express my deepest gratitude to my parents, Abid and Ummuselma. Their affection and encouragement has helped me succeed in many areas of life. A special thanks to my daughter, Zoe for keeping up a shigh spirit. 


\begin{abstract}
New antimycobacterial molecules that kill non-replicating Mycobacterium tuberculosis $(\mathrm{Mtb})$ were identified by screening libraries of synthetic natural products. De novo screening of a 400-membered library of aurachin RE analogs resulted in discovery of UT-317 ((R)-20). UT-317 is a selective vitamin $\mathrm{K}_{2}$ biosynthesis (MenA) inhibitor that killed replicating and non-replicating $M t b$ at $2.31 \mu \mathrm{g} / \mathrm{mL}$ (MIC) and $0.85 \mu \mathrm{g} / \mathrm{mL}$, respectively. A 50-membered library of capuramycin analogs was evaluated in their enzymatic inhibitory activities against translocase I (MraY/MurX) and prenyl-phosphateGlcNAc-1-phosphate transferase (WecA). UT-01320 (45) is identified as a selective WecA inhibitor that kills both replicating and non-replicating $M t b$ at $1.50 \mu \mathrm{g} / \mathrm{mL}$ (MIC) and $2.58 \mu \mathrm{g} / \mathrm{mL}$, respectively. UT-01320 killed the intracellular Mtb much faster than the first-line TB drugs such as isoniazid and rifampicin. A strong antimycobacterial agent, UT-800 (64) was identified by screening of a 50-membered library of pleuromutilin derivatives. UT-800 is a protein biosynthesis (50s ribosome) inhibitor which has activity focused against $M t b$. UT-800 killed replicating and non-replicating $M t b$ at $0.83 \mu \mathrm{g} / \mathrm{mL}$ (MIC) and $1.20 \mu \mathrm{g} / \mathrm{mL}$, respectively. In the course of these works, fluorescent probes, Park's nucleotide- $N^{\varepsilon}-\mathrm{C}_{6}$-FITC (32, for MraY/MurX) and UDP-glucosamine-C 6 -FITC (46, for WecA) were developed. These fluorescent probes enable us to screen the polyprenyl-phosphate $N$-acetylhexosamine 1-phosphate transferase enzyme superfamily (e.g. MraY/MurX, WecA, AlgH, and DPAGT1) in high-throughput manner.
\end{abstract}




\section{TABLE OF CONTENTS}

CHAPTER 1. INTRODUCTION .................................................................................

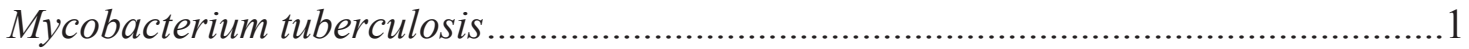

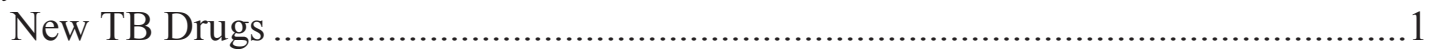

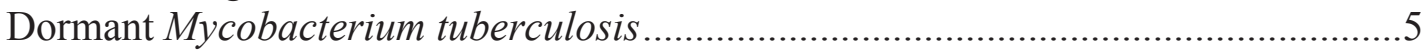

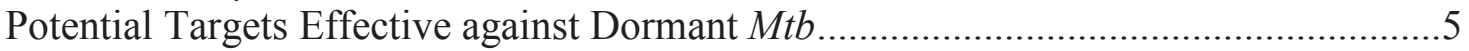

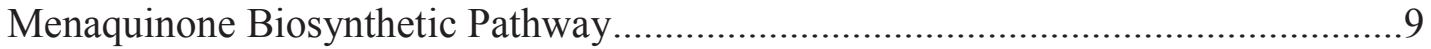

Cell Wall Biosynthesis..................................................................................... 11

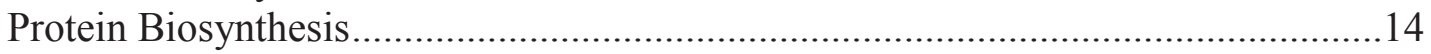

Dissertation Hypothesis and Specific Aims......................................................... 14

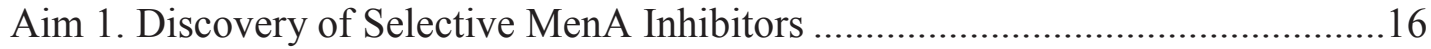

Aim 2. Discovery of WecA-type Phosphotransferase Inhibitors.............................16

Aim 3. Discovery of New 50S Ribosome Inhibitors: Antimycobacterial

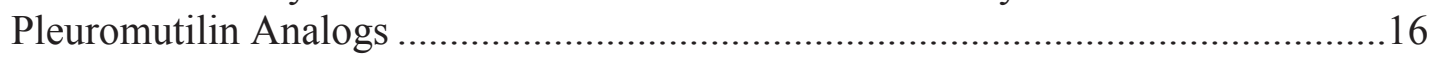

CHAPTER 2. MENAQUINONE BIOSYNTHETIC PATHWAY .............................18

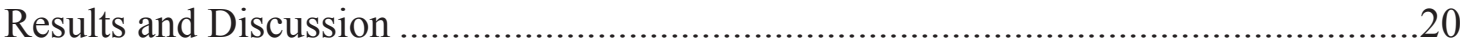

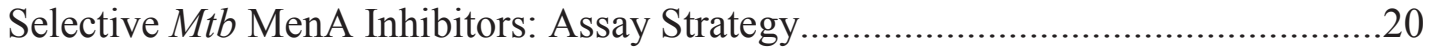

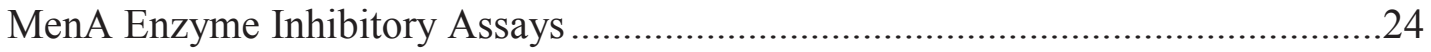

Bacterial Growth Inhibitory Assays.......................................................................24

E. coli Growth Inhibitory Assays under Anaerobic Conditions .............................25

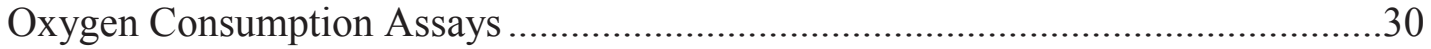

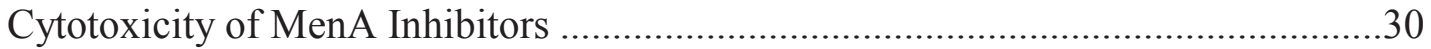

Antibacterial Activities of $(R)-19$ and $(R)-20$ against Drug-resistant $M t b$ and

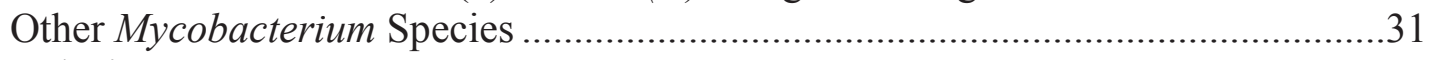

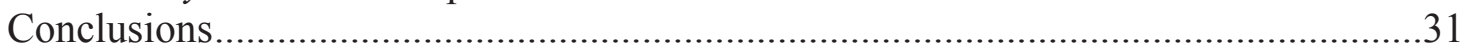

CHAPTER 3. CELL WALL BIOSYNTHESIS INHIBITORS .................................34

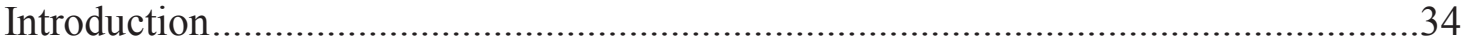

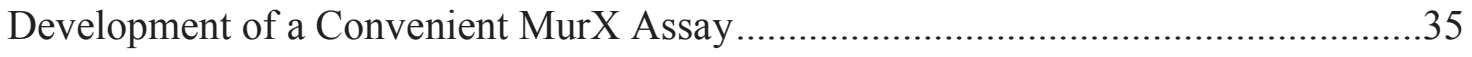

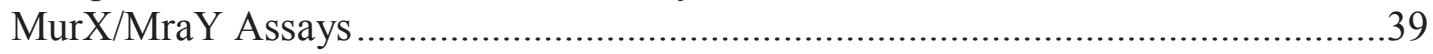

Validation of MurX Assays with Antimycobacterial Uridyl Peptides .....................42

Development of a Convenient WecA Transferase Assay ...........................................44

Specificity of WecA against Nucleotide Substrate and Optimization of Reaction

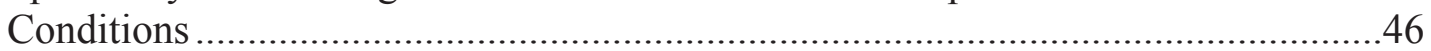

Development of UV/Vis Spectroscopy-based Assay for WecA...............................50

Specificity of WecA against the Structures of Prenyl Phosphate .............................50

Screening a Library of Capuramycin-based Analogs via the New UV-VIS- based

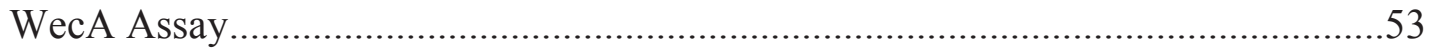

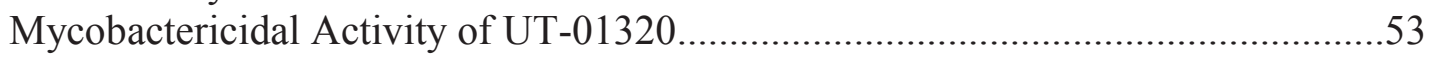

Effectiveness of UT-01320 against Intracellular Mtb .......................................55

Rapid Antimycobactericidal Activity of UT-01320 ........................................5

Synergistic Effect of UT-01320 with MurX Inhibitors .........................................58

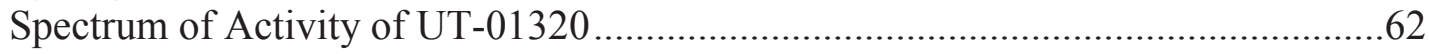


Conclusions

\section{CHAPTER 4. NOVEL PLEUROMUTILIN ANALOGS AS PROTEIN BIOSYNTHESIS INHIBITORS}

Introduction .65

Results and Discussion .68

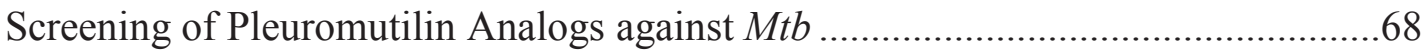

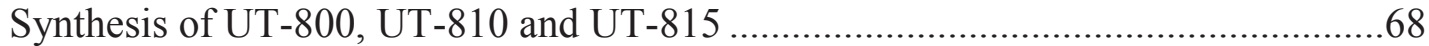

Effectiveness of UT-800, 810 and 815 against Replicating and Non-replicating

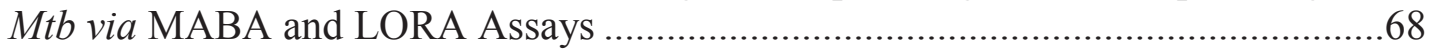

Effectiveness of UT- 800, 810 and 815 against Intracellular Mtb ...........................72

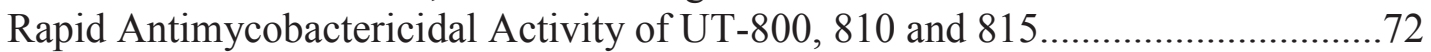

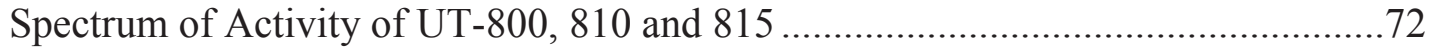

In vitro Metabolic Stability of UT-800, UT-810, and UT-815 ..............................75

Prediction of Intestinal Permeability of UT-800 via Caco-2 Permeability Assay..... 75

Genetic Analysis of UT-490 to Study the Resistance Mechanism ...........................75

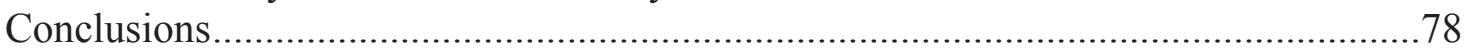

CHAPTER 5. CONCLUSIONS AND FUTURE DIRECTIONS ............................79

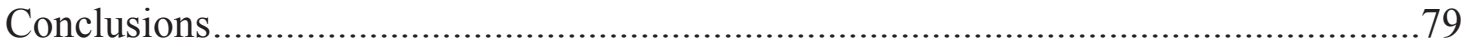

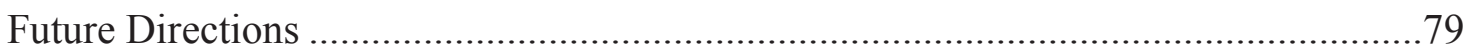

CHAPTER 6. MATERIALS AND METHODS.....................................................82

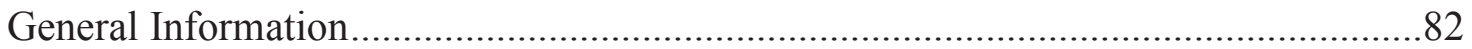

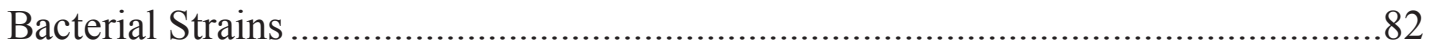

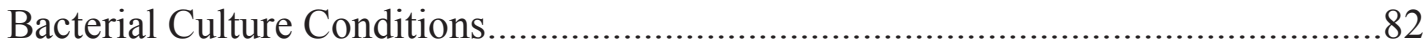

Screening and Assay Development Procedures......................................................83

Minimum Inhibitory Concentration Assays .............................................................83

Microplate Alamar Blue Assay (MABA) ..................................................... 83

Luminescence-based Low-oxygen-recovery Assay (LORA) ............................ 83

MIC on Nutrient Starved Mtb (Loebel Model Assay)...................................... 83

E. coli Growth Inhibitory Assays under Anaerobic Conditions ..............................84

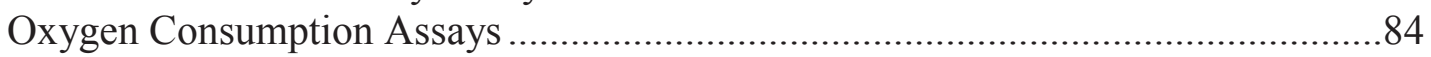

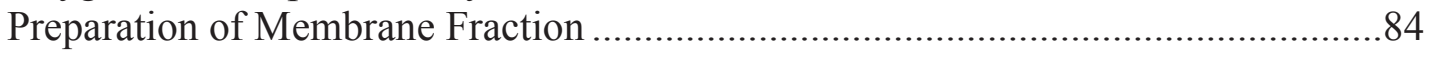

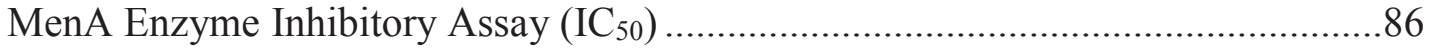

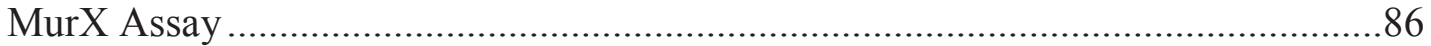

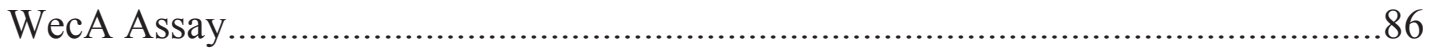

Kill-curve Graph: Determination of Colony Forming Units per Milliliter ................88

Determination of Cytotoxicity $\left(\mathrm{IC}_{50}\right)$ in Vero and $\mathrm{HepG}_{2}$ Cells ..............................8

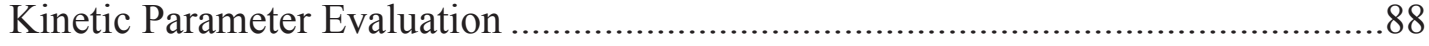

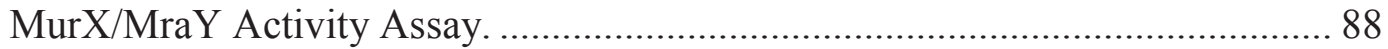

WecA Activity Assay. .............................................................................. 90

Preparation of Polyprenyl Phosphates for MurX and WecA Assays........................90

Synergistic Effect of UT-01320 (45) with SQ 641 (37) or Capuramycin (36).........90

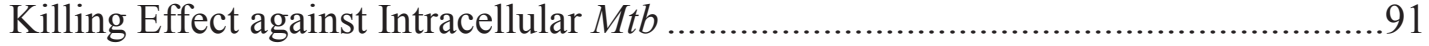

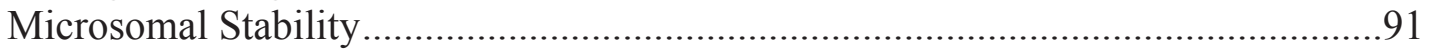


Gene Analyses of Resistant Strain of A. baumannii against UT-490 (68)

LIST OF REFERENCES ...................................................................................96

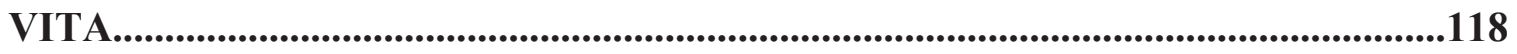




\section{LIST OF TABLES}

Table 1-1. FDA-approved TB drugs and their molecular targets.................................

Table 1-2. New TB drug leads in different stages of clinical trial. ...............................4

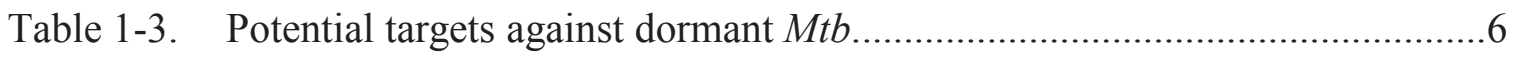

Table 2-1. Antibacterial activities against $M t b / S$. aureus, and MenA enzyme inhibitory activities of all selected molecules. .........................................26

Table 2-2. Activity of compounds against E. coli under anaerobic conditions.............28

Table 2-3. MICs of selected compounds against Mycobacterium spp.........................32

Table 3-1. MurX-catalyzed syntheses of lipid I analogs from $\mathrm{N}$-glycolyl- and $\mathrm{N}$ -

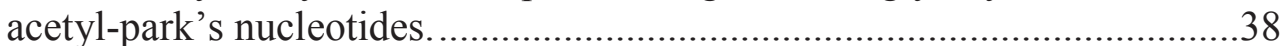

Table 3-2. Assay of positive- and negative-controls, and a library of uridyl peptides against MurX.

Table 3-3. Assays against a library of capuramycin analogs against the WecA enzyme (P-60 from E. coli B21WecA).

Table 3-4. MICs of selected compounds against Mycobacterium spp.

Table 3-5. Fractional inhibitory concentration of a combination of two inhibitor molecules.

Table 3-6. Spectrum of activity of UT-01320.

Table 4-1. Anti-Mtb activity and cytotoxicity of pleuromutilin analogs.

Table 4-2. Spectrum of activity of UT-800, UT-810 and UT-815. .74

Table 6-1. $\quad$ EFIC index for the wells at growth - no growth interface 


\section{LIST OF FIGURES}

Figure 1-1. Cell wall of Mycobacterium tuberculosis in comparison with Grampositive and Gram-negative bacteria.............................................................2

Figure 1-2. Electron transport system and menaquinone biosynthetic pathway.............10

Figure 1-3. Menaquinone biosynthesis inhibitors. ....................................................12

Figure 1-4. Biosynthesis of peptidoglycan and mycolyl-arabinogalactan in $M t b \ldots \ldots \ldots . . .13$

Figure 1-5. Mode of action of protein biosynthesis inhibitors against Mtb....................15

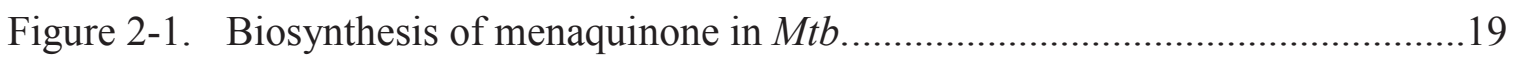

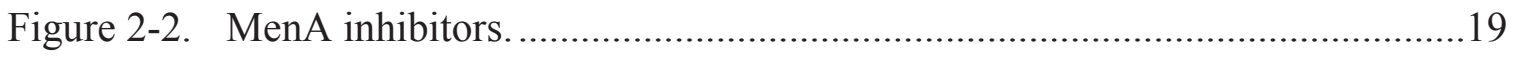

Figure 2-3. Screening strategy for selective MenA inhibitor drug leads against $M t b . . . .21$

Figure 2-4. Topology of $\mathrm{N}$ atom in Mtb selective compounds.....................................22

Figure 2-5. Optically active compounds and their derivatives.....................................23

Figure 2-6. E. coli growth inhibitory assays under anaerobic conditions. .......................29

Figure 2-7. Optically active compounds with selective anti $M t b$ activity.......................32

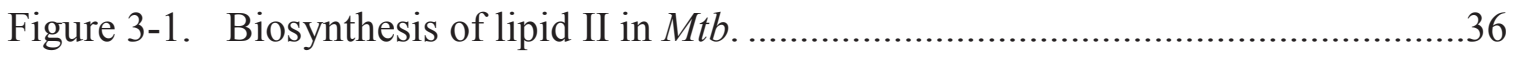

Figure 3-2. MurX-catalyzed syntheses of lipid I analogs from $N$-glycolyl- and $N$ acetyl-park's nucleotides...............................................................................

Figure 3-3. MurX-catalyzed biosynthesis of neryl lipid I analog....................................40

Figure 3-4. HPLC chromatogram of park's nucleotide- $N^{\varepsilon}$-fluorescent analogs (29 and 32) and neryl lipid I- $N^{\varepsilon}$-fluorescent analogs (33 and 34)......................41

Figure 3-5. Library of uridyl peptides utilized for screening against MurX enzyme......43

Figure 3-6. WecA-catalyzed biosynthesis of $\mathrm{C}_{50}$-P-P-Glucosamine- $\mathrm{C}_{6}$-fluorescent analogs 48 and 49 .

Figure 3-7. WecA-catalyzed biosynthesis of $\mathrm{C}_{50}$-P-P-Glucosamine-C 6 -FITC.................49

Figure 3-8. Validation of WecA assays via uv-vis. ......................................................51

Figure 3-9. WecA inhibitors identified in this assay..................................................52 
Figure 3-10. Comparison of specificity of MurX and WecA against prenyl

phosphates.

Figure 3-11. Dose-response curves for the WecA inhibitory activities of UT-01320 and tunicamycin.

Figure 3-12. Structures of capuramycin (36), SQ 641 (37), UT-01320 (45), and UT01309 (42) and summary of SAR obtained from a 100-membered library. .56

Figure 3-13. Bactericidal activity of UT-01320 against non-replicating $M t b$ evaluated in Wayne and nutrient deficient models.

Figure 3-14. Effect of UT-01320 and representative TB drugs against intracellular $M t b$ CDC1551-tdTomato (a transformant Mtb CDC1551 containing tdTomato) in macrophages (J774A.1 cells).

Figure 3-15. In vitro time-kill assessment of capuramycin, 45 and the first-line TB drugs (RIF and INH). .60

Figure 4-1. Representative structures of pleuromutilin analogs. .66

Figure 4-2. Binding sites of pleuromutilin derivatives and linezolid on 50s ribosome...67

Figure 4-3. General structures of pleuromutilin derivatives and Anti-Mtb molecules....69

Figure 4-4. Scheme 1: syntheses of pleuromutilin analogs UT-800, UT-810 and UT815. .70

Figure 4-5. Effect of pleuromutilin analogs and representative TB drugs against intracellular Mtb CDC1551-tdTomato (a transformant Mtb CDC1551 containing tdTomato) in macrophages ( $\mathrm{J} 774 \mathrm{~A} .1$ cells).

Figure 4-6. In vitro time-kill assessment of valnemulin (55), 64, 65, 66 and the firstline TB drugs (RIF and INH).

Figure 4-7. In vitro metabolic stability of pleuromutilin analogs $(64,65$, and 66$) \ldots \ldots . . .76$

Figure 4-8. In vitro Caco-2 permeability assay to predict intestinal permeability of UT-800 (64)...... .77

Figure 4-9. The amino acid alignment of the 50S ribosomal protein L3 (RplC) from a UT-490 (68)-Resistant $A$. baumannii strain (68R). .78

Figure 5-1. Drug leads identified against dormant $M t b$ and novel fluorescent probes for the assay of phosphotransferases. . .80

Figure 6-1. E. coli growth inhibitory assays under anaerobic conditions. .85 


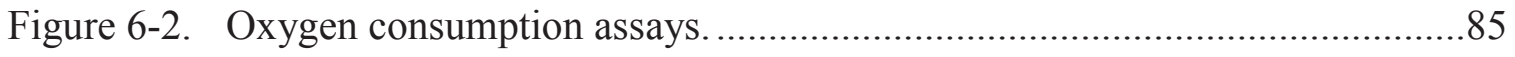

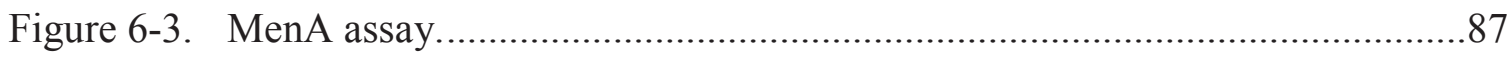

Figure 6-4. Cytotoxicities $\left(\mathrm{IC}_{50}\right)$ of selected compounds with activity against $\mathrm{Mtb}$ $\mathrm{H}_{37} \mathrm{Rv}$ in Vero monkey kidney cells and $\mathrm{HepG}_{2}$ cells..............................89

Figure 6-5. Checkerboard combinations of UT-01320 with SQ641 or Capuramycin. ...92

Figure 6-6. Identification of resistant mechanism of $A$. baumannii to 68 ......................95 


\section{LIST OF ABBREVIATIONS}

\begin{tabular}{|c|c|}
\hline AA-A & Allylaminomethanone-A \\
\hline $\mathrm{ADC}$ & Albumin, Dextrose, Catalase \\
\hline ATCC & American Type Culture Collection \\
\hline ATP & Adenosine Tri Phosphate \\
\hline BDQ & Bedaquiline \\
\hline $\mathrm{CFU}$ & Colony Forming Units \\
\hline CFZ & Clofazimine \\
\hline CoQ & Coenzyme Q (Ubiquinone) \\
\hline CPS & Capsular Polysaccharides \\
\hline DAPI & 4',6-DiAmidino-2-PhenylIndole \\
\hline DHNA & Di Hydro Naphthoic Acid \\
\hline DMF & Di Methyl Formamide \\
\hline DMMK & De Methyl Menaquinone \\
\hline DMSO & Di Methyl Sulfoxide \\
\hline EDTA & Ethylene Diamine Tetra Acetic Acid \\
\hline EMEM & Eagle`s Minimum Essential Medium \\
\hline ETS & Electron Transport System \\
\hline FBS & Fetal Bovine Serum \\
\hline FDA & Food and Drug Administration \\
\hline FIC & Fractional Inhibitory Concentration \\
\hline FITC & Fluorescein Iso Thio Cyanate \\
\hline GlcNAc & N- Acetyl Glucosamine \\
\hline
\end{tabular}




$\begin{array}{ll}\text { GPT } & \text { UDP-GlcNAc:dolichyl-P transferase } \\ \text { HCMI } & \text { Hydrazine Carboximidamide } \\ \text { HIV } & \text { Human Immunodeficiency Virus } \\ \text { HPLC } & \text { High Power Liquid Chromatography } \\ \text { HTS } & \text { High- Throughput Screening } \\ \text { INH } & \text { Isoniazid } \\ \text { LC-MS } & \text { Liquid Chromatography-Mass Spectrometry } \\ \text { LIN } & \text { Linezolid } \\ \text { LORA } & \text { Low Oxygen Recovery Assay } \\ \text { LTBI } & \text { Latent Tuberculosis Infection } \\ \text { MABA } & \text { Microplate Alamar Blue Assay } \\ \text { MDR-TB } & \text { Multi Drug Resistant Tuberculosis } \\ \text { MIC } & \text { Minimum Inhibitory Concentration } \\ \text { MK } & \text { Nuclear Magnetic Resonance Spectroscopy } \\ \text { MKH } 2 & \text { Menaquinone } \\ \text { MOPS } & \text { Menaquinol } \\ \text { MOX } & \text { 4-Morpholine Propane Sulfonic Acid } \\ \text { MRSA } & \text { Moxifloxacin } \\ \text { Mtb } & \text { Methicillin Resistant Staphylococcus aureus } \\ \text { MTT } & \text { NurNGing }\end{array}$




$\begin{array}{ll}\text { OADC } & \text { Oleic Acid, Albumin, Dextrose, Catalase } \\ \text { OBR } & \text { Optimum Background Regimen } \\ \text { OD } & \text { Optical Density } \\ \text { PEG } & \text { Poly Ethylene Glycol } \\ \text { P2HPT } & \text { Polyprenyl-Phosphate N-Acetyl Hexosamine 1-Phosphate } \\ \text { PMBN } & \text { Transferase Superfamily } \\ \text { PTC } & \text { Polymyxin B Nonapeptide } \\ \text { PYZ } & \text { Peptidyl Transferase Center } \\ \text { RIF } & \text { Pyrazinamide } \\ \text { RNAP } & \text { Rifampicin } \\ \text { SAR } & \text { RNA Polymerase } \\ \text { SCV } & \text { Structural Activity Relationship } \\ \text { TB } & \text { Small Colony Variant } \\ \text { TDR-TB } & \text { Tuberculosis } \\ \text { THF } & \text { Totally Drug Resistant Tuberculosis } \\ \text { TLC } & \text { Tetrahydro Furan } \\ \text { UDP } & \text { Thin Layer Chromatography } \\ \text { UMP } & \text { Uridine Di Phosphate } \\ \text { UV-VIS } & \text { Vitrine Mono Phosphate } \\ \text { VK } & \text { World Health Organization } \\ \text { XHO } & \text { Erug }\end{array}$




\section{CHAPTER 1. INTRODUCTION}

\section{Mycobacterium tuberculosis}

Mycobacterium tuberculosis (Mtb) is an acid-fast bacterium that is responsible for tuberculosis (TB). The bacteria infect the lungs (pulmonary TB), but can infect any part of the body such as the kidney, spine, and brain. The reports of verified cases of TB are increasing worldwide, and TB remains one of the top ten causes of death. The World Health Organization (WHO) reported 1.4 million TB-related deaths and 10.4 million new TB incidents in the year 2015 alone (1). The upsurge in the global occurrence of multidrug-resistant TB (MDR-TB) and extreme drug resistant TB (XDR-TB) complicates the TB chemotherapy (2-4). The emergence of totally drug-resistant TB (TDR-TB) has also been reported in Iran, India and South Africa (5-7).

The current treatment regimen for drug-sensitive TB is a combination of four first-line drugs (rifampicin, ethambutol, isoniazid and pyrazinamide) for six months. The MDR-TB treatment is continued up to 20-28 months with first- and second-line drugs. It takes even longer to treat XDR-TB (8). Therefore, it is crucial to discover new drugs that can greatly shorten and/or simplify treatment regimen for drug-resistant strains of Mtb. The development of new TB drugs remains a difficult task. Mtb exists in different physiological states in hosts, and its tolerance to general antibacterial agents make TB drug discovery very challenging (9). Mtb has a thick cell wall which is composed of peptidoglycans, polysaccharides, glycolipids, and mycolic acid (Figure 1-1) (10,11). Mtb cell wall forms an impermeable barrier to many organic molecules including biopolymers (11). Such robust cell wall construction is essential for $M t b$ to promote intracellular survival within macrophages $(12,13)$. The high rates of asymptomatic $M t b$ are due to the fact that $M t b$ can exist as the latent TB (LTB) for many years. In LTB infections, $M t b$ is

present within the granulomas in alveoli by entering a dormant state $(1,14)$. Therefore, the discovery of small molecules that kill the dormant state of $M t b$ at low concentrations is an important subject in TB drug development.

\section{New TB Drugs}

In the last four decades, only two new drugs have been approved for the treatment of Mtb infections. U.S. FDA approved bedaquiline in 2012, and delamanid was given the conditional approval by the European agency in 2013 for MDR-Mtb infections $(15,16)$. Table 1-1 summarizes the FDA-approved anti-tuberculosis drugs and their mode of actions. Novel regimens including new and repurposed drugs are being evaluated in different phases of clinical trials for the treatment of drug-susceptible and/or drugresistant TB $(17,18)$. In the pipeline of TB drugs, only two drug leads belong to new drug classes which exhibit a novel mode of action (Table 1-2). Q-203 (Qurient), a cytochrome bc1 complex inhibitor, entered phase I clinical trial. PA-824 (Pathogenesis Corp/Chiron) is a nitroimadazole that kills $M t b$ through intracellular nitric oxide release (19-21). 


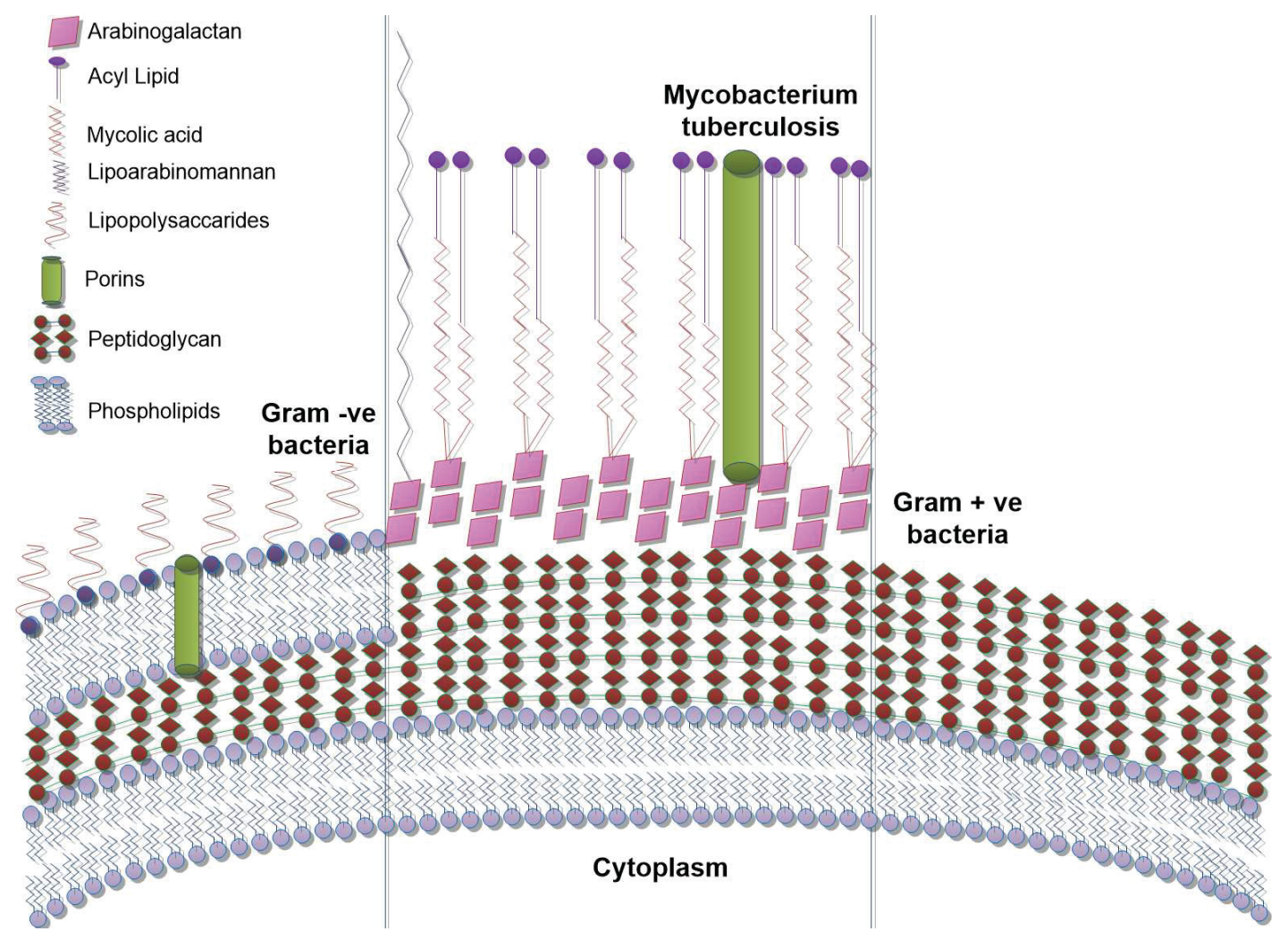

Figure 1-1. Cell wall of Mycobacterium tuberculosis in comparison with Grampositive and Gram-negative bacteria. 
Table 1-1. FDA-approved TB drugs and their molecular targets.

\begin{tabular}{|c|c|c|c|}
\hline Use & Chemical Class & Name & Molecular Target \\
\hline \multirow[t]{4}{*}{ First Line } & Rifamycin & Rifampicin & Protein Biosynthesis \\
\hline & Nicotinic acid & Isoniazid & Mycolic acid biosynthesis \\
\hline & Pyrazine & Pyrazinamide & Increases acidic $\mathrm{pH}$ \\
\hline & Ethylenediamine & Ethambutol & $\begin{array}{l}\text { Arabinogalactan } \\
\text { biosynthesis }\end{array}$ \\
\hline \multirow[t]{9}{*}{ Second Line } & Fluoroquinolones & Ofloxacin & DNA Gyrase \\
\hline & & Ciprofloxacin & DNA Gyrase \\
\hline & Aminoglycosides & Amikacin & Protein Biosynthesis \\
\hline & & Kanamycin & Protein Biosynthesis \\
\hline & & Capreomycin & Protein Biosynthesis \\
\hline & Isoxazolidinone & Cycloserine & Peptidoglycan \\
\hline & Nicotinamide & Ethionamide & Mycolic acid biosynthesis \\
\hline & Aminosalicylic acid & $\begin{array}{l}\text { Para aminosalicylic } \\
\text { acid }\end{array}$ & Folic acid biosynthesis \\
\hline & Oxazolidinone & Linezolid & Protein Biosynthesis \\
\hline \multirow{2}{*}{$\begin{array}{l}\text { Other } \\
\text { Drugs }\end{array}$} & Diarylquinolone & Bedaquiline & ATP Synthase \\
\hline & $\begin{array}{l}\text { Nitroimidazole } \\
\text { Riminophenazine }\end{array}$ & $\begin{array}{l}\text { Delamanid } \\
\text { Clofazimine }\end{array}$ & Mycolic acid biosynthesis \\
\hline
\end{tabular}


Table 1-2. New TB drug leads in different stages of clinical trial.

\begin{tabular}{|c|c|c|c|}
\hline Name & Chemical Class & Molecular Target & Phase \\
\hline PA-824 & Nitroimidazole & Intracellular NO release & Phase III \\
\hline Q-203 & Imidazopyridine & Cytochrome bc1 (ETS) & Phase I \\
\hline SQ-109 & Ethylenediamine & Mycolic biosynthesis & Phase II $^{\mathrm{a}}$ \\
\hline Meropenem & Carbapenem & Cell wall biosynthesis & Phase II $^{\mathrm{a}}$ \\
\hline Faropenem & Carbapenem & Cell wall biosynthesis & Phase II $^{\mathrm{a}}$ \\
\hline High dose rifampicin & Rifamycin & RNA polymerase & Phase III \\
\hline Linezolid & Oxazolidinone & 50S ribosome & Phase III \\
\hline Sutezolid & Oxazolidinone & 50S ribosome & Phase IIa $^{\mathrm{a}}$ \\
\hline AZD5847 & Oxazolidinone & 50S ribosome & Phase $\mathrm{IIa}^{\mathrm{a}}$ \\
\hline Moxifloxacin & Fluoroquinolone & DNA replication & Phase III \\
\hline Gatifloxacin & Fluoroquinolone & DNA replication & Phase II $^{\mathrm{a}}$ \\
\hline Levofloxacin & Fluoroquinolone & DNA replication & Phase III \\
\hline Metronidazole & & DNA replication & Phase II $^{\mathrm{a}}$ \\
\hline PA824-MOX-PYZ & - & - & Phase II $^{\mathrm{a}}$ \\
\hline PA824-BDQ-LIN & - & - & Phase III \\
\hline Delamanid-OBR & - & - & Phase III \\
\hline
\end{tabular}

NOTE: PYZ-Pyrazinamide, MOX-Moxifloxacin, BDQ-Bedaquiline, LIN-Linezolid, OBR-Optimized background regimen, ${ }^{\text {a }}$ Completed. 
Although a phase III trial of PA-824 was initiated in combination with moxifloxacin and pyrazinamide, an unexpected suspension of the clinical trial was reported due to toxicity (22,23). In May 2016, the TB Alliance noted that the trial may restart after making necessary addition of safety procedures (22). A different combination of PA-824, bedaquiline and linezolid is currently being evaluated in clinical studies $(22,24)$. SQ-109 (25) reached a phase II trial but the clinical studies were discontinued due to lack of discernable advantage in efficacy over the control group (18,22,25-27). A phase II trial is completed for meropenem/faropenem plus amoxicillin and clavulanic acid. This trial evaluated the rapid bactericidal activity, safety and tolerability in patients with pulmonary tuberculosis (24). A clinical trial will assess the maximum tolerated dose of rifampicin and the rapid killing effect of higher doses of rifampicin against tubercle bacillus in the lungs (24). Novel oxazolidinones and fluoroquinolones have progressed to clinical studies (24). Metronidazole is aimed to develop into a second-line therapy for MDR-TB, and it was evaluated in a phase II study (24). The need for new TB drugs is obvious, and treatment regimens need to be simplified by introduction of new drugs that are effective against MDR-Mtb and their dormant state.

\section{Dormant Mycobacterium tuberculosis}

$M t b$ is a facultative aerobic bacterium which can also survive under anaerobic conditions. $M t b$ is thought to exist in a non-replicating (dormant) form particularly inside the caseous nodules of host lung granulomas where it has very little access to oxygen and nutrients (28-31). Mtb remains in the dormant form in these caseous pulmonary nodules for decades. In patients with compromised immune systems due to HIV/AIDS, the nonreplicating bacilli are reactivated and progress the disease $(32,33)$. The dormant form of $M t b$ shows resistance to many clinically used TB drugs. It is believed that the eradication of $M t b$ dormant form requires long-term chemotherapy for tuberculosis (34). If a drug effectively kills the dormant form at low concentrations, such a drug would have a significant effect on the duration of TB therapy.

\section{Potential Targets Effective against Dormant $M t b$}

Dr. Kurosu published a comprehensive review of the potential targets effective against dormant $M t b$ (35). Based on the reported genetic experiments and effects of selective inhibitor molecules on the molecular targets, the genes associated with DNA gyrases, reductases (that produce toxic materials via activation of prodrugs), protein kinases, DosS/DosR systems, glyoxylate shunt, methyltransferases, electron transport systems, ATP synthase, menaquinone biosynthesis, protein biosynthesis, and cell wall biosynthesis remain actively expressed in dormant $M t b$ cells.

Table 1-3 summarizes investigational drug leads and FDA-approved drugs that affect the potential molecular targets for dormant $M t b$. Fluoroquinolones, DNA gyrase inhibitors, were reported to kill dormant Mtb in vitro. Moxifloxacin and gatifloxacin 
Table 1-3. Potential targets against dormant $M t b$.

\begin{tabular}{|c|c|c|c|c|}
\hline Potential Targets & Drug Leads & MIC replicating $M t b$ & MIC $_{\text {non-replicating } M t b}$ & References \\
\hline \multirow{7}{*}{$\begin{array}{l}\text { Protein } \\
\text { biosynthesis }\end{array}$} & Pleuromutilins $^{\mathrm{a}}$ & $0.78-1.56 \mu \mathrm{g} / \mathrm{mL}^{\mathrm{a}}$ & $1.04-1.98 \mu \mathrm{g} / \mathrm{mL}^{\mathrm{a}, \mathrm{b}}$ & \\
\hline & Rifampicin & $0.05-0.2 \mu \mathrm{g} / \mathrm{mL}$ & $1.5 \mu \mathrm{g} / \mathrm{mL}^{\mathrm{b}}$ & $(47)$ \\
\hline & Sutezolid & $0.2-0.4 \mu \mathrm{g} / \mathrm{mL}$ & $1 \mu \mathrm{g} / \mathrm{mL}^{\mathrm{b}}$ & (48) \\
\hline & DA-7157 & $0.125-0.5 \mu \mathrm{g} / \mathrm{mL}$ & ND & (49) \\
\hline & Spectinamides & $0.8-1.6 \mu \mathrm{g} / \mathrm{mL}$ & $0.06 \%$ viable cfu $/ \mathrm{mL}$ at $10 \mu \mathrm{g} / \mathrm{mL}^{\mathrm{b}}$ & $(50)$ \\
\hline & Linezolid & $<0.125-4 \mu \mathrm{g} / \mathrm{mL}$ & $65.2 \mu \mathrm{M}^{\mathrm{b}}$ & $\begin{array}{c}(47,48,51,5 \\
2)\end{array}$ \\
\hline & AZD 5847 & $0.125-4 \mu \mathrm{g} / \mathrm{mL}$ & $1 \mu \mathrm{g} / \mathrm{mL}^{\mathrm{b}}$ & $(48,53)$ \\
\hline \multirow[t]{6}{*}{ DNA gyrase } & Moxifloxacin & $0.125 \mu \mathrm{g} / \mathrm{mL}$ & $15.9 \mu \mathrm{g} / \mathrm{mL}^{\mathrm{b}}$ & $(47,52,54)$ \\
\hline & Gatifloxacin & $0.06 \mu \mathrm{g} / \mathrm{mL}$ & $2 \mu \mathrm{g} / \mathrm{mL}^{\mathrm{b}}$ & $(55)$ \\
\hline & Mannich Base derivative & $8-16 \mu \mathrm{g} / \mathrm{mL}^{\mathrm{c}}$ & ND & $(56)$ \\
\hline & Pyrrolamide derivatives & $0.03-0.25 \mu \mathrm{g} / \mathrm{mL}$ & ND & (57) \\
\hline & AB-1 & $0.06 \mu \mathrm{g} / \mathrm{mL}$ & $1 \mu \mathrm{g} / \mathrm{mL}^{\mathrm{b}}$ & (58) \\
\hline & Thiazolopyridone ureas & $0.1 \mu \mathrm{M}$ & ND & (59) \\
\hline Methyltransferase & - & - & - & \\
\hline $\begin{array}{l}\text { DosS/DosR } \\
\text { systems }\end{array}$ & Phenylcoumarines & - & $\begin{array}{l}>99 \% \text { reduction in viability at } \\
131 \mu \mathrm{g} / \mathrm{mL}\end{array}$ & $(60)$ \\
\hline \multirow[t]{6}{*}{ Protein kinase } & Benzo[g]quinoxaline & ND & ND & $(61)$ \\
\hline & AX20017 & $\begin{array}{l}6-15 \mu \mathrm{g} / \mathrm{mL} \\
99 \% \text { inhibition } 10 \mu \mathrm{M}^{\mathrm{d}}\end{array}$ & ND & $(61-64)$ \\
\hline & VI-17494 & $20-50 \mu \mathrm{M}^{\mathrm{d}}$ & ND & $(61,63)$ \\
\hline & VI-17499 & $20-50 \mu \mathrm{M}^{\mathrm{d}}$ & ND & $(61,63)$ \\
\hline & VI-437 & $20-50 \mu \mathrm{M}^{\mathrm{d}}$ & ND & $(61,63)$ \\
\hline & MRT67127 & $8-16 \mu \mathrm{M}$ & ND & $(65)$ \\
\hline
\end{tabular}


Table 1-3. (Continued).

\begin{tabular}{|c|c|c|c|c|}
\hline Potential Targets & Drug Leads & MIC replicating $M t b$ & $\mathrm{MIC}_{\text {non-replicating } M t b}$ & References \\
\hline \multirow{5}{*}{$\begin{array}{l}\text { Glyoxylate } \\
\text { pathway }\end{array}$} & Mannich Base derivative & ND & ND & (66) \\
\hline & 3-nitropropionamides & $0.16-3.3 \mu \mathrm{M}$ & $0.04-1.72 \mu \mathrm{M}^{\mathrm{e}}$ & $(67,68)$ \\
\hline & Phenyl-diketo acids & $<1-16 \mu \mathrm{M}$ & ND & (69) \\
\hline & salicylanilide & $0.25-8 \mu \mathrm{M}$ & ND & (70) \\
\hline & IMBI-3 & $0.25-0.5 \mu \mathrm{g} / \mathrm{mL}$ & ND & (71) \\
\hline \multirow{3}{*}{$\begin{array}{l}\text { Activation via } \\
\text { reductases }\end{array}$} & Metronidazole & $>1000 \mu \mathrm{M}$ & $8.56 \mu \mathrm{M}^{\mathrm{b}}$ & $(72,73)$ \\
\hline & PA-824 & $0.015-0.25 \mu \mathrm{g} / \mathrm{mL}$ & $2-10 \mu \mathrm{g} / \mathrm{mL}^{\mathrm{b}}$ & $(19,21,74)$ \\
\hline & Delamanid & 0.012 & $0.7-4.4 \mu \mathrm{g} / \mathrm{mL}^{\mathrm{b}}$ & $(75,76)$ \\
\hline \multirow{5}{*}{$\begin{array}{l}\text { Electron transport } \\
\text { systems }\end{array}$} & Chlorpromazine & $0.23-3.6 \mu \mathrm{g} / \mathrm{mL}^{\mathrm{d}}$ & - & $(77,78)$ \\
\hline & Trifluoperazine & $10 \mu \mathrm{g} / \mathrm{mL}$ & $5 \mu \mathrm{g} / \mathrm{mL}^{\mathrm{f}}$ & (79) \\
\hline & Thioridazine & $2-4 \mu \mathrm{g} / \mathrm{mL}^{\mathrm{d}}$ & $7.41-18.53 \mu \mathrm{g} / \mathrm{mL}^{\mathrm{b}}$ & $(80-82)$ \\
\hline & & & $18.53 \mu \mathrm{g} / \mathrm{mL}^{\mathrm{e}}$ & \\
\hline & Clofazimine & $0.15-2.5 \mu \mathrm{g} / \mathrm{mL}$ & $0.8-1.5 \mu \mathrm{g} / \mathrm{mL}^{\mathrm{b}}$ & (47) \\
\hline \multirow{4}{*}{$\begin{array}{l}\text { Menaquinone } \\
\text { biosynthesis }\end{array}$} & $\mathrm{UT}-317^{\mathrm{a}}$ & $2.31 \mu \mathrm{g} / \mathrm{mL}^{\mathrm{a}}$ & $0.85 \mu \mathrm{g} / \mathrm{mL}^{\mathrm{a}, \mathrm{b}}$ & (83) \\
\hline & $\begin{array}{l}\text { 7-methoxy-2-naphthol } \\
\text { derivatives }\end{array}$ & $3-5 \mu \mathrm{g} / \mathrm{mL}$ & ND & (84) \\
\hline & $\begin{array}{l}\text { Methyl and phenyl- } \\
\text { butanoates }\end{array}$ & $0.64 \mu \mathrm{g} / \mathrm{mL}$ & $1.5 \mu \mathrm{g} / \mathrm{mL}^{\mathrm{b}}$ & $(85,86)$ \\
\hline & Sulfonyl adenosine analogs & ND & ND & $(87,88)$ \\
\hline $\mathrm{F}_{1} \mathrm{~F}_{0}$ ATP Synthase & Bedaquiline & $0.002-0.06 \mu \mathrm{g} / \mathrm{mL}$ & $1-2.78 \mu \mathrm{g} / \mathrm{mL}^{\mathrm{b}}>11.11 \mu \mathrm{g} / \mathrm{mL}^{\mathrm{e}}$ & $(82,89)$ \\
\hline \multirow{2}{*}{$\begin{array}{l}\text { Cell wall } \\
\text { biosynthesis }\end{array}$} & CPZEN 45 & $0.2-1.56 \mu \mathrm{g} / \mathrm{mL}$ & ND & $(90)$ \\
\hline & UT-01320 a & $1.56 \mu \mathrm{g} / \mathrm{mL}^{\mathrm{a}}$ & $2.58 \mu \mathrm{g} / \mathrm{mL}^{\mathrm{a}, \mathrm{b}}$ & $(91)$ \\
\hline
\end{tabular}


Table 1-3. (Continued).

\begin{tabular}{lllll}
\hline Potential Targets & Drug Leads & MIC replicating Mtb & MIC non-replicating $\boldsymbol{M t b}$ & References \\
\hline $\begin{array}{l}\text { Cell wall } \\
\text { biosynthesis }\end{array}$ & Meropenem+Clavulanate & $0.32 \mu \mathrm{g} / \mathrm{mL}^{\mathrm{g}}$ & $0.19 \sim 12.5 \mu \mathrm{g} / \mathrm{mL}^{\mathrm{b}, \mathrm{h}}$ & $(92)$ \\
& $\begin{array}{l}\text { C-2 ester/oxadiazole } \\
\text { substituted cephalosporins }\end{array}$ & $>100 \mu \mathrm{g} / \mathrm{mL}$ & $0.88 \mu \mathrm{g} / \mathrm{mL}^{\mathrm{i}}$ \\
& & & $(93)$ \\
\hline
\end{tabular}

NOTE: a Data generated in this study, ${ }^{\mathrm{b}} \mathrm{MIC}$ obtained on $\mathrm{Mtb}$ cultured in hypoxic conditions, ${ }^{\mathrm{c}}$ MIC determined against $M$. smegmatis, ${ }^{\mathrm{d}} \mathrm{MIC}$ obtained on intracellular Mtb, ${ }^{\mathrm{e}} \mathrm{MIC}$ obtained on nutrient deficient $\mathrm{Mtb},{ }^{\mathrm{f}} \mathrm{MIC}$ obtained on nitric oxide induced $\mathrm{Mtb}$, and bacteriostatic effect was observed at $5 \mu \mathrm{g} / \mathrm{mL}$ concentration, ${ }^{\mathrm{g}} \mathrm{MIC}$ of meropenem with $5 \mu \mathrm{g} / \mathrm{mL}$ clavulanate, ${ }^{\mathrm{h}} \mathrm{MIC}$ of meropenem with $2.5 \mu \mathrm{g} / \mathrm{mL}$ clavulanate, ${ }^{\mathrm{i}} \mathrm{MIC}$ obtained on multi stress model of non-replicating $M t b$, ND - Not determined. 
progressed to clinical development for use in combination with the other TB drugs (rifampicin, isoniazid and pyrazinamide)(24,36). PA-824 is thought to exhibit activity against dormant $M t b$ by activation via reductases $(19,20,35)$. Ser/Thr protein kinases, PknA, B and G play an essential role in signal transduction in dormant $M t b(35,37)$. DosS/DosR, two component regulatory systems in $M t b$ are known to be important for its virulence and is responsible for the regulation of the dormancy state $(35,38,39)$. The glyoxylate shunt is another pathway that was reported to be upregulated during dormancy (40).

Intracellular $M t b$ requires an alternative carbon metabolism because of the scarcity of carbohydrate and amino acid nutrients inside infected cells. Mtb in macrophages uptakes simple carbon compounds as carbon sources through the glyoxylate shunt mechanism (41). The key enzymes, malate synthase and isocitrate lyase have been studied in this pathway. Protein biosynthesis pathways have great potential to be effective targets for dormant $M t b$. Menaquinone biosynthetic pathway is evaluated as a critical component for the survival of $M t b$ due to the fact that it directly links to the ATP production in both replicating and non-replicating $M t b$. Although the importance of the cell wall for intracellular survival of $M t b$ has been discussed, the cell wall biosynthesis has not yet been established as a target for anti-dormant $M t b$.

\section{Menaquinone Biosynthetic Pathway}

Lipoquinones are involved in the electron transport system (ETS) as electron carriers for the synthesis of ATP. Mtb and Gram-positive bacteria utilize menaquinone (MK) in their ETS (42). Gram-negative bacteria use ubiquinone (CoQ) in their ETS in aerobic conditions and menaquinone in anaerobic conditions $(43,44)$ (Figure 1-2A and B). Humans utilize ubiquinone and lack a menaquinone biosynthetic pathway. Dhiman et al. have demonstrated that menaquinone synthesis is critical for mycobacterial growth, and its biosynthetic pathway presents a viable drug target (45). Although dormant Mtb is known to have less active metabolism and energy reserves, the ATP synthesis should be active in the dormant mycobacteria (46). Murphy et al. demonstrated that eight gene clusters involved in $M t b$ electron transport are upregulated in dormant Mtb (94). The ETS couples with $\mathrm{F}_{1} \mathrm{~F}_{0}$ ATPase to produce ATP through oxidative phosphorylation; thus, the menaquinone biosynthetic pathway could be a selective target against $M t b$. The biosynthetic pathway of menaquinone utilizes seven Men enzymes (MenA-MenG) (Figure 1-2C). Menaquinone biosynthesis begins with conversion of chorismate to isochorismate by MenF. MenD catalyzes the addition of $\alpha$-ketoglutarate to isochorismate to form 2-succinyl-6-hydroxy-2,4-cyclohexadiene-1-carboxylate. Subsequent steps catalyzed by MenC, MenE and MenB form 1,4-dihydroxy-2-naphthoate (DHNA). MenA (1,4-dihydroxy -2-naphthoate prenyl transferase) catalyzes the decarboxylative prenylation of DHNA to form demethylmenaquinone (DMMK). MenG catalyzes methylation of DMMK with S- adenosyl L- methionine (SAM) to complete the synthesis of MK. MenA is a membrane-associated protein that is predicted to have five 


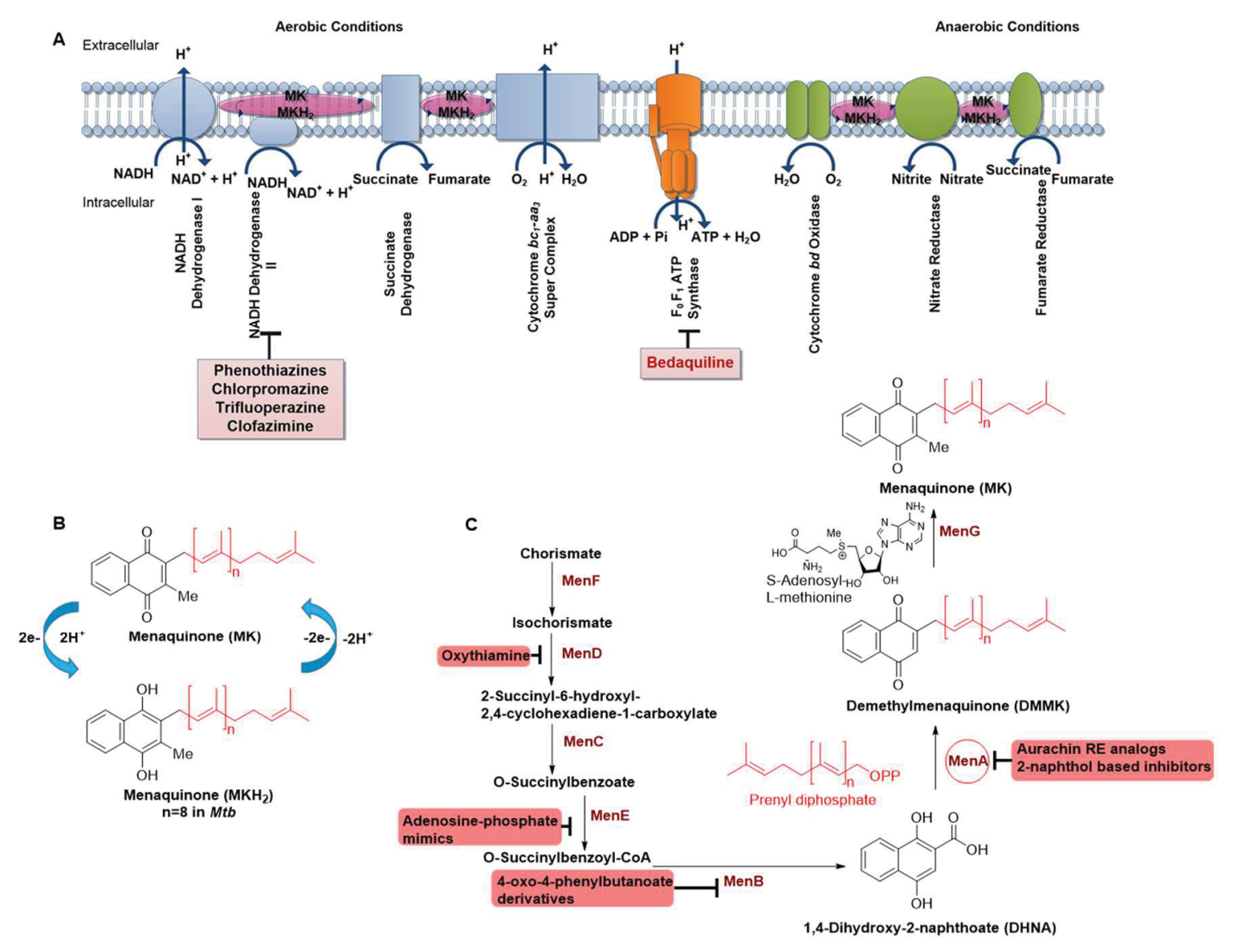

Figure 1-2. Electron transport system and menaquinone biosynthetic pathway. A) Electron transport system couples with $\mathrm{F}_{1} \mathrm{~F}_{0}$ ATPase to produce ATP in Mtb. Phenothiazines inhibit NADH dehydrogenase II and bedaquiline inhibit $\mathrm{F}_{1} \mathrm{~F}_{0}$ ATPase. Menaquinone is the only lipoquinone used as an electron carrier in Mtb ETS. B) MK transfers two electrons in ETS for the ATP synthesis and reduces to menaquinol $\left(\mathrm{MKH}_{2}\right)$. C) Menaquinone (MK) biosynthetic pathway: MenA is inhibited by our aurachin RE analogs. 
transmembrane segment by SOSUI (95). Our group has studied the function of the MenA enzyme towards the development of new drug leads against MDR-Mtb and dormant $M t b$ (45,96-98).

To date, several groups have identified menaquinone biosynthesis inhibitors and validated MenB, MenE, and MenA as drug targets for development of new antibacterial agents (Figure 1-3). Lu et al. reported a series of adenosine-phosphate mimics which showed MenE enzyme inhibitory activity $(87,88)$. Matarlo et al. demonstrated that the 4oxo-4-phenylbutanoate derivatives are effective inhibitors of MenB and exhibit antibacterial activity against MRSA and $M t b(86)$. Xu et al. identified that an oxythiamine derivative is an anti-Staphylococcal MenD inhibitor (99).

MenA is the sixth enzyme of the MK pathway. Our group has pioneered design and synthesis of a series of selective MenA inhibitors $(83,97)$. The identified MenA inhibitors showed significant growth inhibitory activities against drug-resistant Grampositive bacteria including $M t b$. Significantly, the selective MenA inhibitors killed nonreplicating $M t b$ at equal or lower to/than the MIC concentrations for replicating $M t b$ $(93,96)$. Choi et al. reported 2-naphthol-based MenA inhibitors with bactericidal activity against $M t b$ and methicillin-resistant S. aureus (MRSA) (84)

\section{Cell Wall Biosynthesis}

The thick cell wall of $M t b$ is known to play an important role in its intracellular survival $(100,101)$. Peptidoglycan (PG) biosynthesis has been extensively studied, but only a few enzymes associated with PG biosynthesis (e.g. transpeptidase of penicillin binding proteins (PBPs) and MurA) were of interest to the drug industry for antibacterial drug discovery. Comparisons with gene expression studies of $M t b$ at the exponential phase and the non-replicating state indicated that the genes associated with extracellular materials (arabinosyl transferases, lipoarabinomannan biosynthesis, mycolic acid biosynthesis, and other enzymes associated with reconstructions of cell walls) are upregulated in dormant $M t b$ (102). Therefore, inhibition of the committed steps of the mycolyl-arabino galactan biosynthesis will affect the intracellular growth and dormancy of $M t b$ cells. We have studied polyprenyl-phosphate N-acetylhexosamine-1-phosphate transferase enzyme superfamily (P2HPT) (103). In Mtb, WecA catalyzes the first membrane-associated step that anchors the phospho-GlcNAc moiety of UDP-GlcNAc to decaprenyl phosphate $\left(\mathrm{C}_{50}-\mathrm{P}\right)$, leading to the formation of mycolyl-arabino galactan (104) (Figure 1-4). We expect that inhibition of WecA could impair the structure of the mycolyl-arabino galactan in $M t b$, significantly increasing intracellular killing effect in macrophages or epithelial cells.

WecA homologs include MraY/MurX, TagO, WbcO, WbpL, RpgG, WbaP and eukaryotic UDP-GlcNAc:dolichyl-phosphate transferase (GPT) (105-107). MraY/MurX catalyzes the first membrane-anchored step in PG biosynthesis (108). MurX is specific to UDP-N-acetyl muramyl-pentapeptide (Park's nucleotide), forming decaprenyl- 


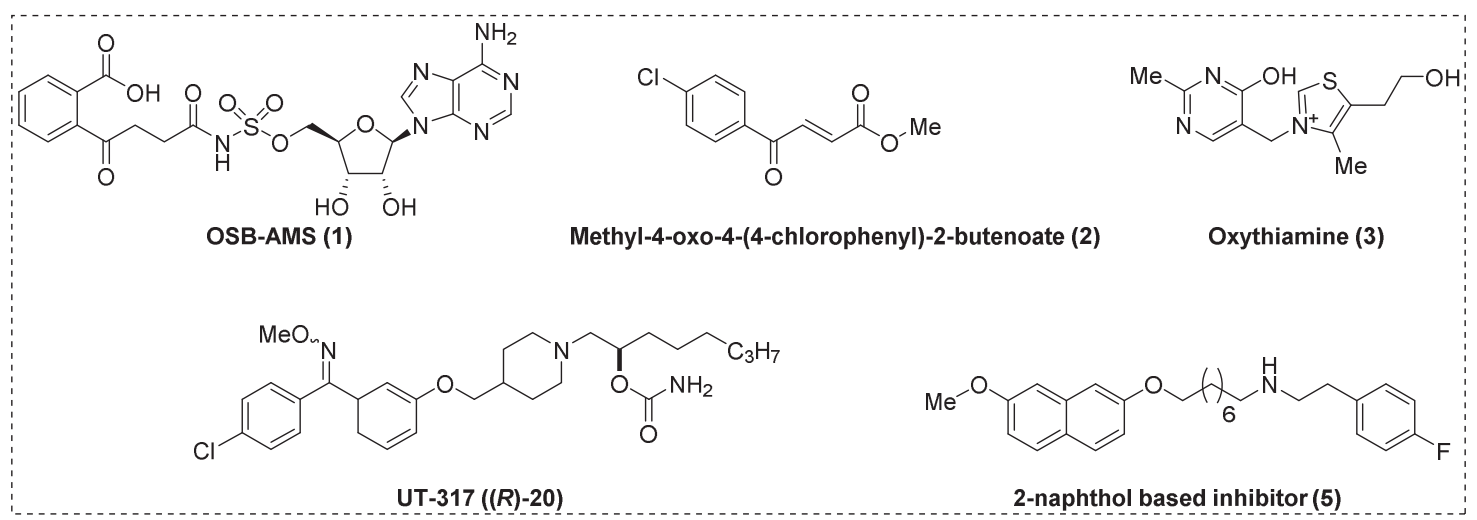

Figure 1-3. Menaquinone biosynthesis inhibitors.

1 - The MenE inhibitor reported by Lu et al, 2 - The MenB inhibitor reported by Matarlo et al, 3 - The MenD inhibitor reported by Xu et al, $(\boldsymbol{R})-\mathbf{2 0}$ - A menA inhibitor, This study (Chapter 2), 5 - The MenA inhibitor reported by Choi et al. 


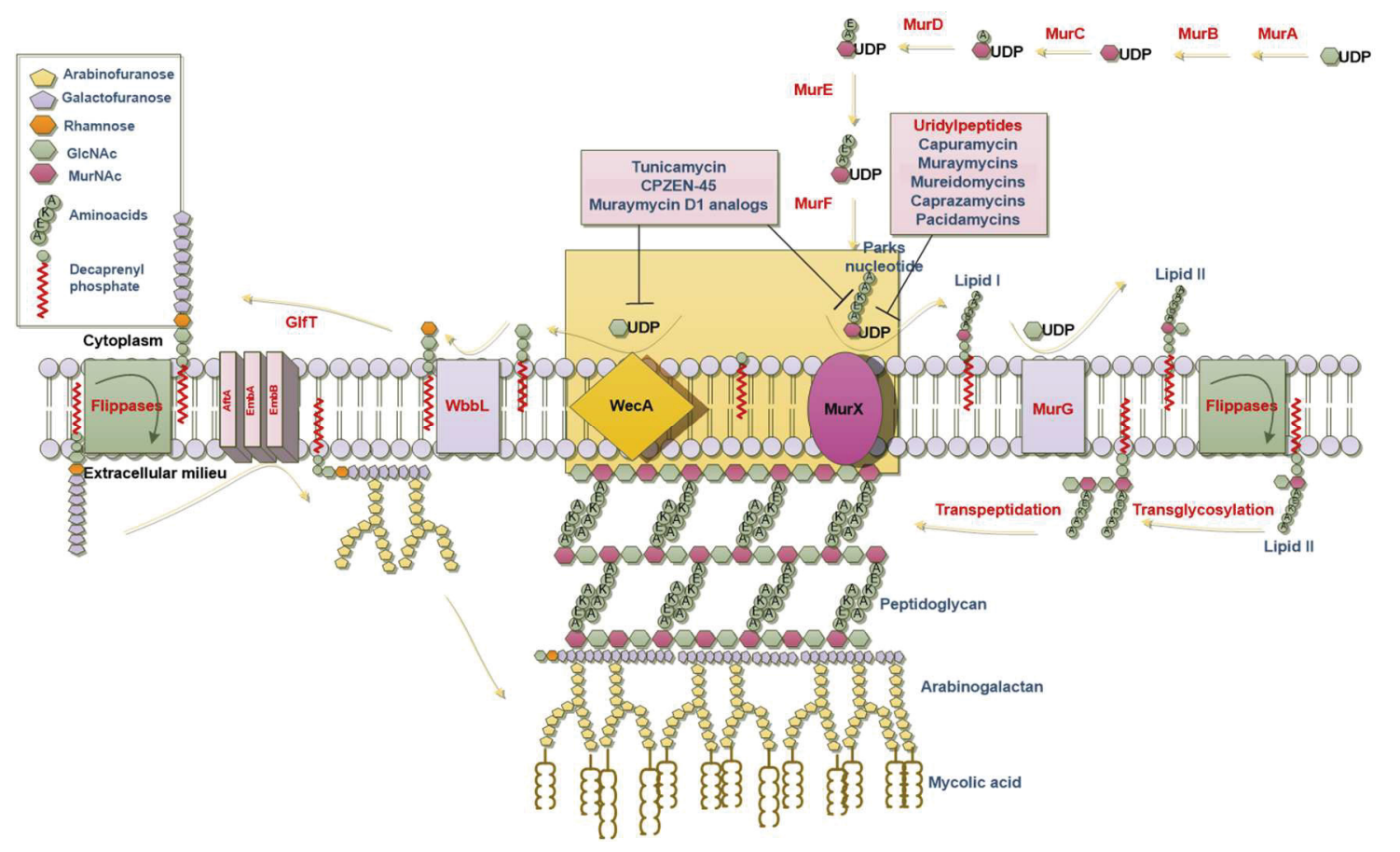

Figure 1-4. Biosynthesis of peptidoglycan and mycolyl-arabinogalactan in Mtb. MurX and WecA utilize decaprenyl phosphate. MurX catalyzes transformation from Parks nucleotide to lipid I, while WecA transfers from UDP-GlcNAc to $\mathrm{C}_{50}$-P-P-GlcNAc. Tunicamycin, CPZEN-45 and muraymycin D1 are inhibitors of both WecA and MurX. We identified a selective WecA inhibitor, UT-01320 (45). 
diphosphoryl-N-acetylmuramyl-pentapeptide (lipid I) (Figure 1-4). We demonstrated that MurX inhibitors are effective in killing only replicating $M t b$ under aerobic conditions. On the contrary, WecA inhibitors are effective in killing non-replicating $M t b$ under oxygen-depleted conditions. To date, only tunicamycin, CPZEN 45, and muraymycin D1 have been demonstrated to have enzyme inhibitory activity against both MurX and WecA $(90,109,110)$. We identified, for the first time, that a capuramycin derivative, UT-01320 kills non-replicating Mtb by targeting WecA selectively.

\section{Protein Biosynthesis}

Bacterial protein biosynthesis is a clinically validated drug target. However, there is very little information related to the translation steps and dormant form of $M t b$. Rifamycin, DNA-dependent RNA polymerase inhibitor is active against non-replicating $M t b$ at higher concentrations. Aminoglycoside antibiotics bind to the $30 \mathrm{~S}$ bacterial ribosomal subunit that effectively kill non-replicating $M t b$ in vitro. The oxazolidinone class of protein biosynthesis inhibitors (e.g. linezolid and sutezolid) are also effective in killing non-replicating $M t b(48,50,111,112)$. We confirmed effectiveness of antimycobacterial protein biosynthesis inhibitors for non-replicating via the Wayne dormancy model and low oxygen recovery (LORA) assays. Although several protein biosynthesis inhibitors are listed in the second-line TB drugs (e.g. amikacin and capreomycin), the narrow safety margin of FDA-approved protein biosynthesis inhibitor drugs discourage physicians from applying them to treat MDR- and XDR-Mtb infections (113-116).

Pleuromutilin and its derivatives have been studied for development of new antiStaphylococcal infections. To date, very few pleuromutilin derivatives have been developed for MDR-Mtb infections, and their efficacy against non-replicating $M t b$ is not known. Figure 1-5 illustrates the mode of actions of protein biosynthesis inhibitors and the binding site of pleuromutilin in 50s ribosomes. Pleuromutilin interferes with both the A-site and P-site of the 50s ribosomal subunit $(117,118)$. It was evident that the pleuromutilin binding site overlaps only partially with the linezolid (an A-site binder) binding site in the 50s ribosome $(118,119)$. In a cell-based screening of pleuromutilin derivatives against a drug sensitive $M t b(\mathrm{H} 37 \mathrm{Rv})$, several new molecules exhibited bactericidal activity with an MIC of below $1.0 \mu \mathrm{g} / \mathrm{mL}$. The identified derivatives displayed significant effect in killing non-replicating Mtb in vitro.

\section{Dissertation Hypothesis and Specific Aims}

A fundamental problem in the treatment of tuberculosis (TB) is the requirement of long-term treatment with multiple drugs. Because the treatment for latent TB infection is a reason for long duration of therapy, the discovery of a drug that effectively kills nonreplicating (dormant) $M t b$ will have the potential to significantly reduce TB treatment time. During the Ph.D. period (2011-2016), I have studied three drug targets that are 

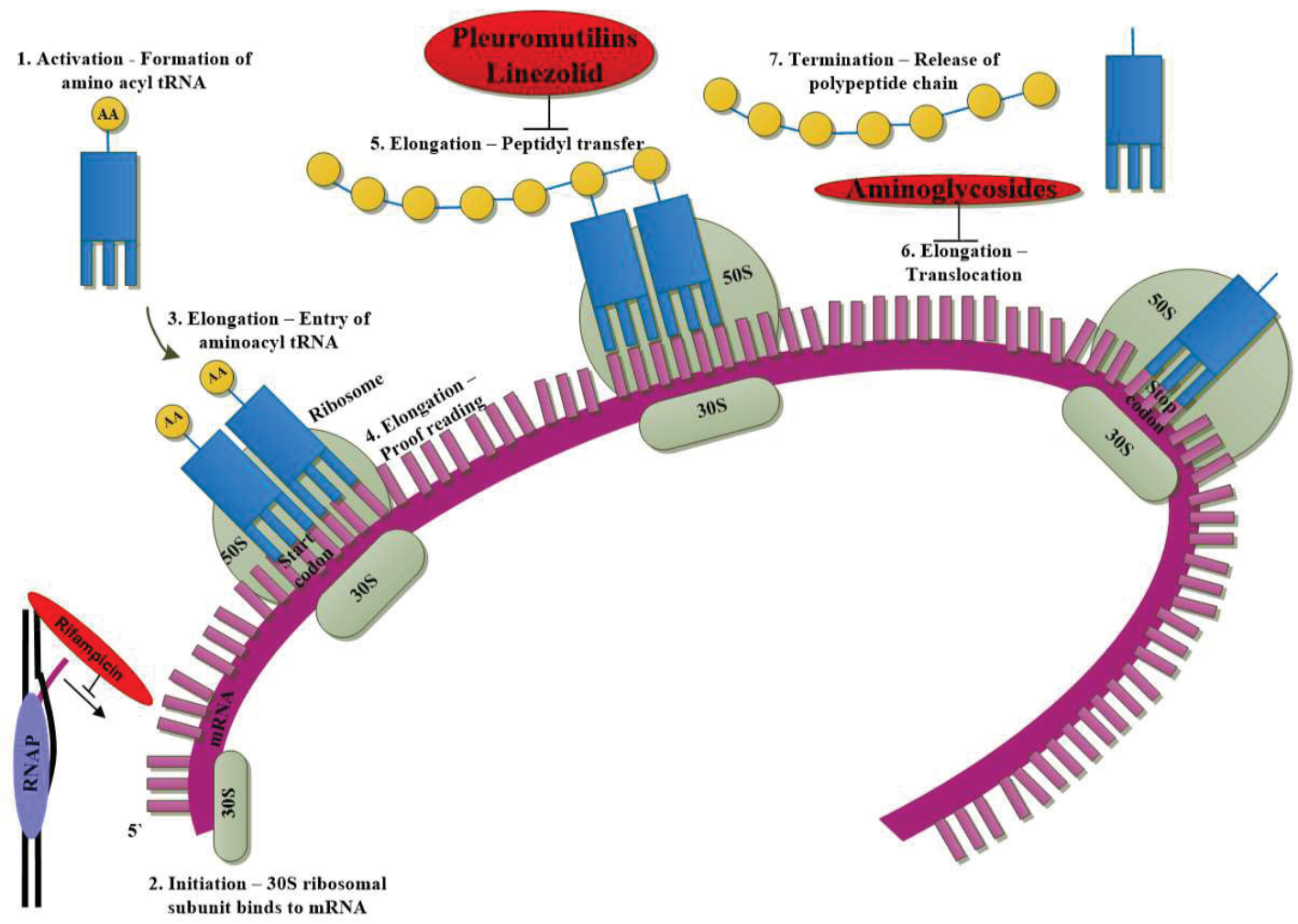

B:

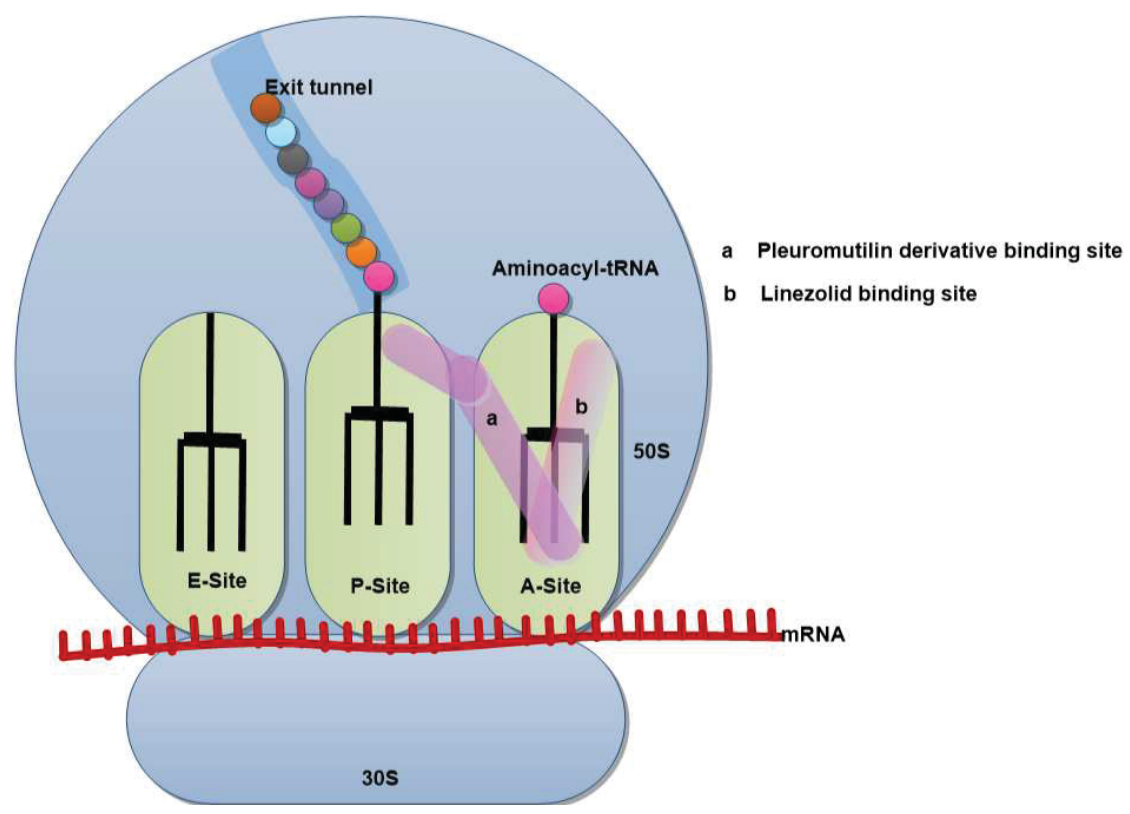

Figure 1-5. Mode of action of protein biosynthesis inhibitors against Mtb. A) Pleuromutilins and linezolid inhibit peptidyl transfer in elongation step. Rifampicin inhibits RNA polymerases (RNAP) and aminoglycosides inhibit ribosomal translocation step. B) Pleuromutilins and linezolid bind to different sites at 50S ribosomal subunit. 
essential for maintaining the viability of dormant form of $M t b$ : menaquinone (vitamin $\mathrm{K}_{2}$ ), mycolylarabinogalactan and protein biosynthetic pathways. The hypothesis was that, validation of selective inhibitors of these molecular targets in our in vitro assays will provide valuable insight into the development of new TB drugs that are effective in killing dormant $M t b$.

\section{Aim 1. Discovery of Selective MenA Inhibitors}

$M t b$ uses menaquinone in both aerobic and anaerobic conditions as an electron carrier for ATP synthesis, while humans solely use ubiquinone. We identified that a quinolone natural product, aurachin RE exhibited MenA enzyme (the sixth enzyme in the menaquinone biosynthetic pathway) inhibitory activity and a broad-spectrum of activity against Gram-positive bacteria including Mtb $(83,120)$. A 400-membered library of aurachin RE analogs available in our group were screened by a de novo assay strategy that can identify selective MenA inhibitors with a narrow spectrum of activity focused against $M t b$.

\section{Aim 2. Discovery of WecA-type Phosphotransferase Inhibitors}

Undecaprenyl-phosphate $\alpha$ - $N$-acetylglucosaminyl 1-phosphate transferase (WecA) enzyme is an essential enzyme for the biosynthesis of mycolyl-arabinogalactan conjugate and growth of Mtb. Maintenance of integrity of the cell wall is important for $M t b$ to survive in macrophages, and thus inhibition of WecA-type phosphotransferase is believed to be effective in killing non-replicating $M t b$.

Capuramycin is a natural product that inhibits peptidoglycan biosynthesis by selectively targeting MurX-type phosphotransferase. We discovered that a chemical modification of capuramycin alters its molecular target and one of the analogs UT-01320 (45) kills non-replicating $M t b$ at low concentrations. In order to identify selective WecA inhibitors, we have developed new WecA- and MurX-type assay methods using the fluorescent probes 32 and 46. Robustness of our fluorescent-based assays is demonstrated by screening a 50 membered-library of capuramycin analogs. Through in vitro profiling of the selective WecA inhibitor $\mathbf{4 5}$ is performed.

\section{Aim 3. Discovery of New 50S Ribosome Inhibitors: Antimycobacterial Pleuromutilin Analogs}

Pleuromutilin analogs exhibit strong 50S ribosome inhibition and have been developed for the infections caused by drug resistant strains of Gram-positive bacteria. In our program of extended spectrum of activity of pleuromutilin, our group has generated a library of new analogs and screens via the MABA and LORA assays. We identify novel pleuromutilin analogs with a narrow spectrum of activity towards $M t b$ that effectively kills replicating and non-replicating $M t b$ at very low concentrations. In vitro and in vivo 
toxicity studies are performed to advance an antimycobacterial pleuromutilin for in vivo efficacy studies using an infected animal model. 


\section{CHAPTER 2. MENAQUINONE BIOSYNTHETIC PATHWAY*}

Function of ubiquinone (coenzyme $\mathrm{Q}_{10}$ ) as a component of the mitochondrial respiratory chain in human is well established ("the chemiosmotic theory", Mitchell, 1978) (121-123). In prokaryotes, especially in Gram-positive bacteria, menaquinone transfers two electrons in a process of either aerobic or anaerobic respiration. On the other hand, a majority of Gram-negative organisms utilize ubiquinone under aerobic conditions and menaquinone under anaerobic conditions in their electron transport systems $(44,124,125)$. Therefore, inhibitors of menaquinone biosynthesis or specific inhibitors of enzymes associated with electron transport systems have great potential for the development of novel and selective drugs against MDR Gram-positive bacteria (96). The electron transport system couples with ATP synthase to produce ATP through oxidative phosphorylation. Bacterial ATP synthase, $\mathrm{F}_{1} \mathrm{~F}_{0}$-ATPase, is a viable target for treatment of MDR Mtb infections. A diarylquinolone, bedaquiline, is an inhibitor of $M t b$ ATP synthase that exhibited a remarkable activity against Mtb (126). However, only few studies have investigated the electron transport system for development of new antibacterial drugs (96). Weinstein and co-workers reported the inhibitors of type II $\mathrm{NADH}$ : menaquinone oxidoreductase that effectively killed Mtb in vitro and they concluded that type II NADH dehydrogenase could be a unique and interesting antimicrobial target (77). We have reported that the inhibition of MenA (1,4-dihydroxy2-naphthoate prenyltransferase), which catalyzes a formal decarboxylative prenylation of 1,4-dihydroxy-2-naphthoate (DHNA) to form demethylmenaquinone (DMMK) in menaquinone biosynthesis (Figure 2-1), showed significant growth inhibitory activities against drug resistant Gram-positive bacteria including Mtb (97,98). In menaquinone biosynthesis, MenD (2-succinyl-5-enoylpyruvyl-6-hydroxy-3-cyclohexane-1-carboxylic acid synthase), MenE (an acyl-CoA synthase), and MenB (1,4-dihydroxynaphthoyl-CoA synthase) have recently been studied for the development of novel drug lead for Grampositive pathogens including $M t b(88,127-129)$.

In an attempt at finding a new pharmacophore for MenA inhibitors, we identified a new quinolone natural product, aurachin RE, 6 (Figure 2-2A) which exhibited MenA enzyme inhibitory activity as well as a wide antimicrobial spectrum against Grampositive bacteria (120). As there is an overall structural resemblance between aurachin $\mathrm{RE}$ and our $1^{\text {st }}$ generation MenA inhibitors ( 7 and $\mathbf{8}$ ) (Figure 2-2B and $\mathbf{C}$ ) or menaquinone, aurachin RE's antibacterial activity could be attributed to a synergistic effect of respiratory chain and menaquinone biosynthesis inhibitory activities. The identification of aurachin RE's MenA enzyme inhibitory activity encouraged us to redesign and synthesize chiral MenA inhibitor molecules, in which primary or secondary alcohol was introduced in the side chain of the $1^{\text {st }}$ generation MenA inhibitor molecules

* Adapted with permission. Debnath, J., Siricilla, S., Wan, B., Crick, D. C., Lenaerts, A. J., Franzblau, S. G., and Kurosu, M. (2012). Discovery of selective menaquinone biosynthesis inhibitors against Mycobacterium tuberculosis. J. Med. Chem. 55 3739-3755. 
<smiles>O=C(O)c1cc(O)c2ccccc2c1O</smiles>

1,4-Dihydroxy-2-naphthoate (DHNA)
MenA

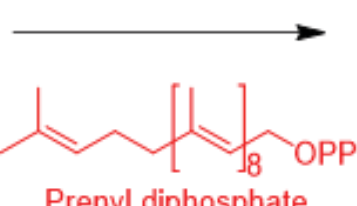

Prenyl diphosphate

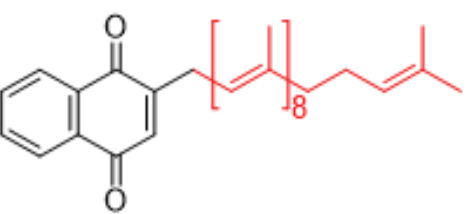

Demethylmenaquinone (DMMK)

Figure 2-1. Biosynthesis of menaquinone in $M t b$.

MenA catalyzes the prenylation of DHNA to form DMMK, this is one step prior to the formation of menaquinone.

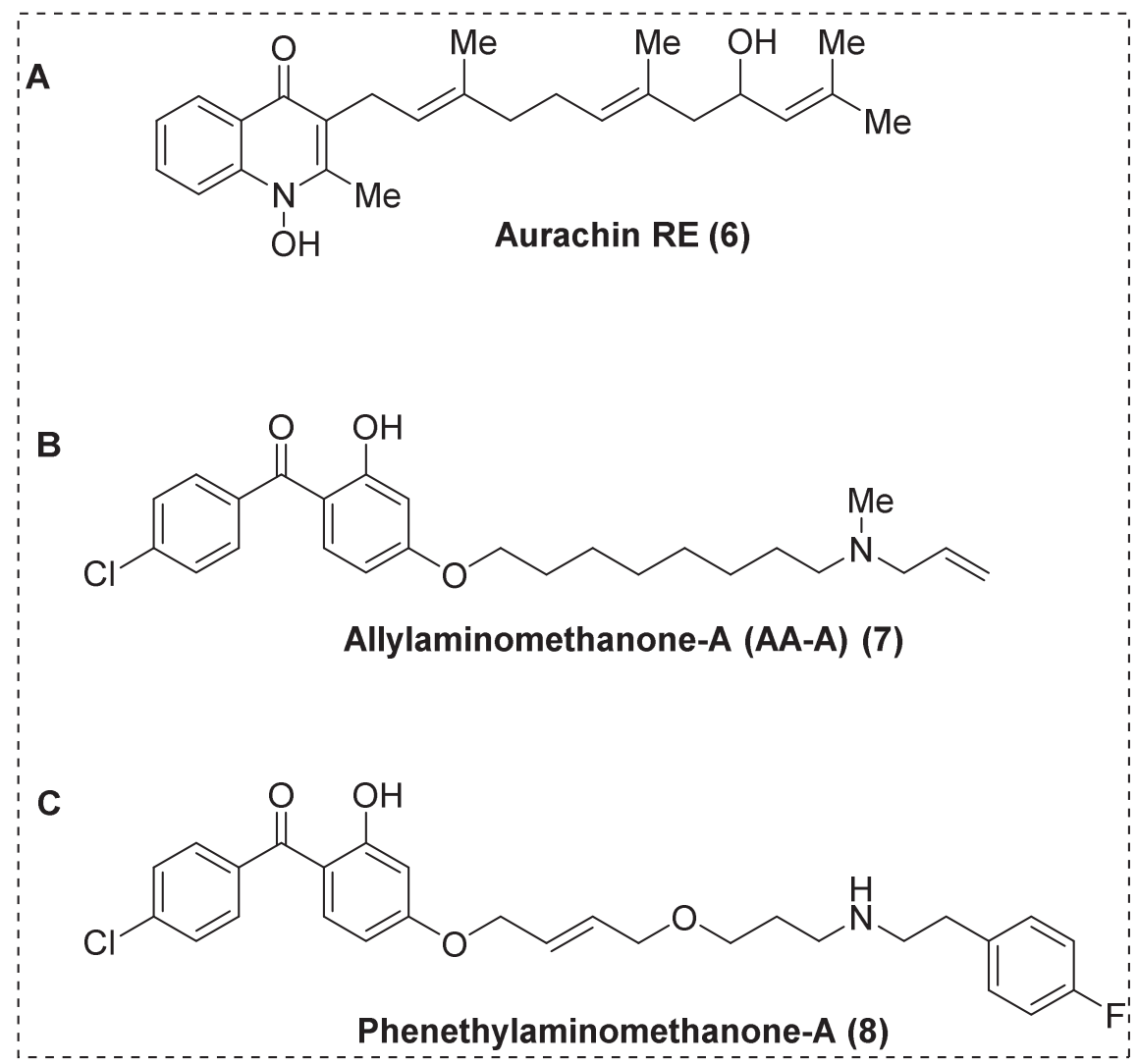

Figure 2-2. MenA inhibitors.

A) Aurachin RE is a natural product with MenA inhibitory activity. B and C) Our first generation MenA inhibitors (7 and 8). 
$(97,98)$. Our lab has generated over 400 molecules with $>95 \%$ purity either in solution or on polymer-support, and these molecules were evaluated in vitro $\left(\mathrm{IC}_{50}\right)$ against menA enzyme and bacterial growth inhibitory assays (38). Figure 2-3 illustrates our assay scheme to identify selective MenA inhibitors against Mtb. Out of these molecules, 26 compounds were identified to exhibit the in vitro biological activities which met the assay criteria summarized in Figure 2-3. Based on obtained SAR from a 400-membered library, it became evident that the topology of the $N$ atom in the inhibitor molecules plays an important role in selectivity of the MenA enzymatic and bactericidal activities ( $M t b$ vs. S. aureus). As summarized in Figure 2-4, selective mycobactericidal molecules (913) possess the secondary or tertiary amine in the near center of the molecules (highlighted moieties in 9-13 in Figure 2-4), whereas the topology of the $N$ atom of the molecules possessing antibacterial activities against both Mtb and $S$. aureus (14-17) locates the right half of the molecules (highlighted moieties in 14-17 in Figure 2-4). We have identified selective antimycobacterial MenA inhibitors in their racemic forms. In order to obtain insight into the effect of chirality of new MenA inhibitors (9-13 in Figure 2-4), we commenced syntheses of the optically active forms of the identified inhibitors (Figure 2-5).

\section{Results and Discussion}

\section{Selective Mtb MenA Inhibitors: Assay Strategy}

Antimicrobial spectrum focused against $M t b$ (selective antimycobacterial agent) is preferable for TB chemotherapy (130). We realized that the peptide sequences of the $M t b$ $m e n A$ and $S$. aureus menA gene products are only 32\% identity and 50\% similarity in the BLAST experiment (131). Indeed, we have identified several molecules that exhibit selective MenA enzyme and bacterial growth inhibitory activities against $M t b$; more than a 10-fold higher inhibitory activity against $M t b$ than $S$. aureus. In order to develop MenA inhibitors which are selective against $M t b$, molecules generated in this program were first evaluated in MenA enzyme inhibitory assays. Only molecules exhibited Mtb MenA activity over $S$. aureus MenA ( $\mathrm{IC}_{50}<20 \mu \mathrm{M},>60 \mu \mathrm{M}$ against $M t b \mathrm{MenA}$ and $S$. aureus MenA, respectively) were evaluated in bacterial growth inhibitory assays (MICs) using $M t b, S$. aureus, and E. coli (Figure 2-3). The molecules exhibited good activity only against Mtb (MICs for Mtb, S. aureus, and E. coli are $<12.5,>60$, and $>125 \mu \mathrm{g} / \mathrm{mL}$, respectively) were evaluated in $E$. coli growth inhibitory assays under anaerobic conditions followed by menaquinone supplementation assays ( $E$. coli utilize only menaquinone in their electron transport system under anaerobic conditions) (43). This assay confirmed that the molecules killed bacteria by targeting MenA enzyme or electron transport systems (vide infra). Oxygen consumption assay using $M t b$ identifies electron transport system inhibitors; in this assay, selective MenA inhibitors should not show activity at concentrations below the MIC against Mtb $(132,133)$. Selective antimycobacterial MenA inhibitors confirmed via E. coli growth inhibitory assays (under anaerobic conditions) and oxygen consumption assays were evaluated for their activity 


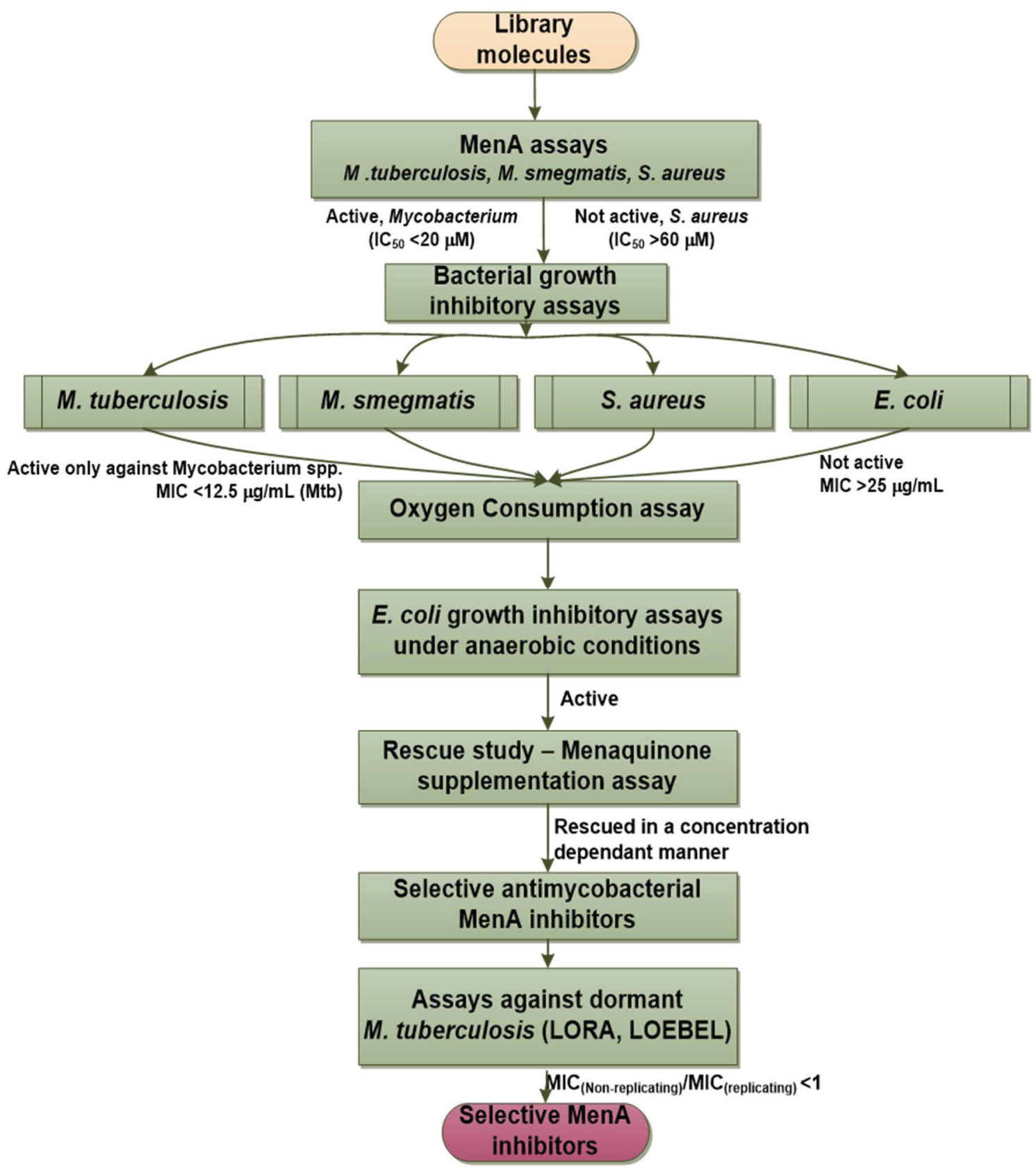

Figure 2-3. Screening strategy for selective MenA inhibitor drug leads against Mtb. 


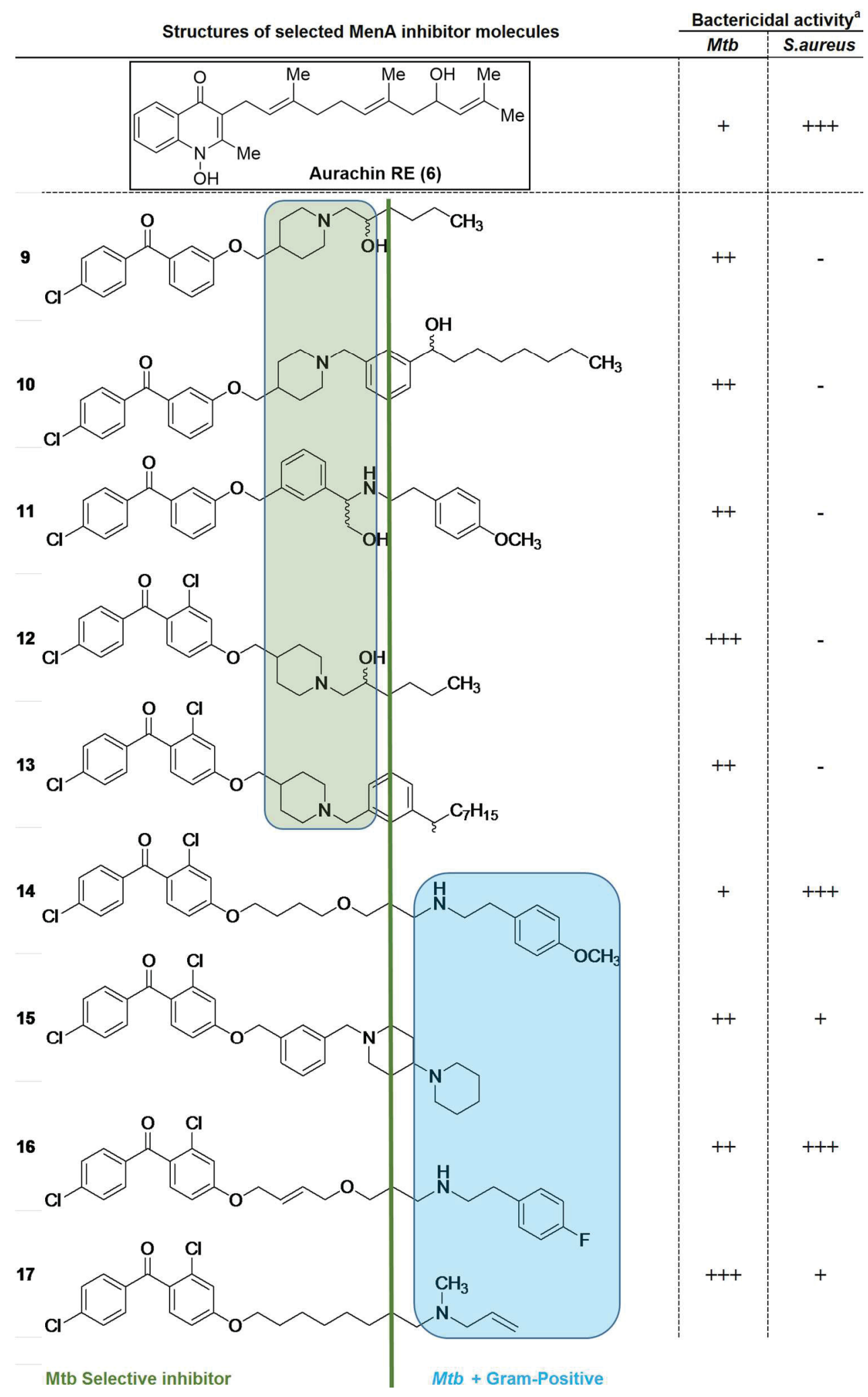

Figure 2-4. Topology of $\mathrm{N}$ atom in $\mathrm{Mtb}$ selective compounds.

$+++(0.5-8.0 \mu \mathrm{g} / \mathrm{mL}),++(8.0-15.5 \mu \mathrm{g} / \mathrm{mL}),+(15.5-30.0 \mu \mathrm{g} / \mathrm{mL}),-(>50 \mu \mathrm{g} / \mathrm{mL})$, ${ }^{a}$ Minimum inhibitory concentration in $\mu \mathrm{g} / \mathrm{mL}$. 


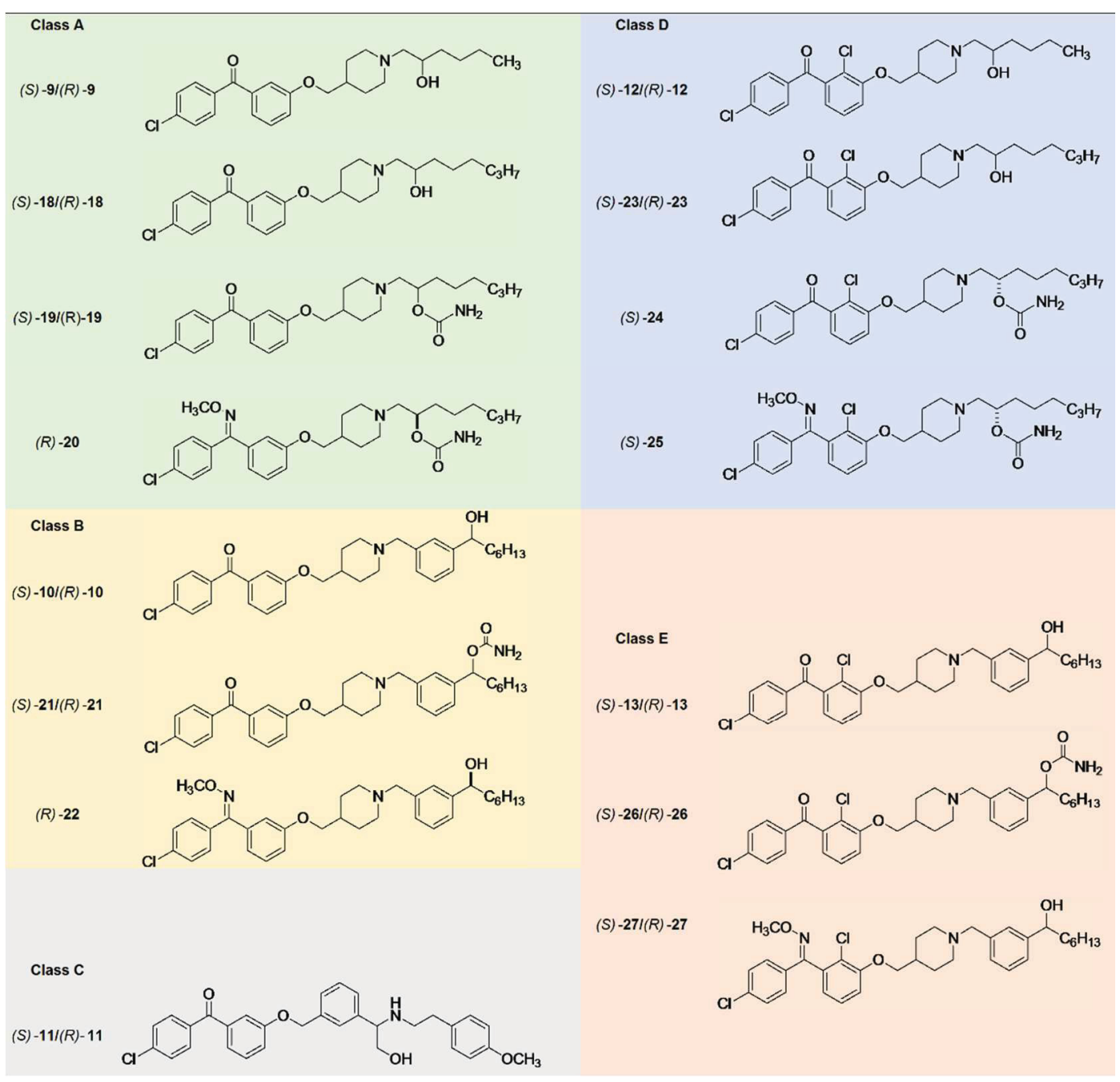

Figure 2-5. Optically active compounds and their derivatives. 
against non-replicating Mtb via the low oxygen recovery assays (LORA) and nutrient deficient model assay (Loebel). LORA assay is an In vitro modification of Wayne's model and was developed by Dr. Scott Franzblau and colleagues of Illinois TB Research Institute, USA (47). LORA assay evaluates potency against non-replicating $M t b$ cells under low oxygen conditions and Loebel model assay measures the potency against nonreplicating $M t b$ cells under nutrient depleted conditions.

\section{MenA Enzyme Inhibitory Assays}

MenA enzymatic assays of generated molecules were originally performed with $\left[{ }^{3} \mathrm{H}\right]$ farnesyl diphosphate and MenA containing membrane fraction (45). This assay requires a significant effort to separate demethylmenaquinone (DMMK) from the reaction mixtures and includes the significant cost for $\left[{ }^{3} \mathrm{H}\right]$ farnesyl diphosphate. In order to simplify the procedure and to reduce the cost of MenA enzyme inhibitory assays, we have developed a MenA assay using HPLC. In new MenA assay developed by our group, the MenA product, demethylmenaquinone can readily be quantitated via UV absorbance (DMMK; $\lambda 2325 \mathrm{~nm}$ ).

We performed a preliminary screening of the activity of compounds synthesized (Figure 2-5) at the single concentrations of $100 \mu \mathrm{M}$. In these MenA enzyme inhibitory assays, the optically active molecules were assayed against Mtb and $S$. aureus MenA. $\mathrm{IC}_{50}$ values of the molecules which exhibited activity against Mtb MenA and inactivity against $S$. aureus MenA were calculated. Of 86 optically active molecules synthesized, 26 molecules exhibited $M t b$ MenA inhibitory activity and were inactive against $S$. aureus MenA at $100 \mu \mathrm{M}$ concentrations. Dose-response plots (DMMK formation vs. concentrations of inhibitor) were obtained for 26 molecules to determine $\mathrm{IC}_{50}$ values. Significant effect of chirality in the MenA enzyme inhibitory activity was observed in the molecules in classes A and D. On the contrary, an obvious effect of chirality in MenA enzyme inhibitory activity of the molecules in classes B, C, and E was not observed; the racemic forms and each enantiomer did not show noticeable difference in MenA enzyme inhibitory activity. It is noteworthy that the carbamate analog $(R)-\mathbf{1 9}$ group showed 6-fold better MenA enzyme inhibitory activity compared to the alcohol form $(R)$-18. Similar improvement of enzymatic inhibition by modification of the alcohol with the carbamate group is observed in the molecules in class D.

\section{Bacterial Growth Inhibitory Assays}

All molecules that showed activity against MenA in a preliminary assay at 100 $\mu \mathrm{M}$ concentrations were evaluated for their mycobacterial growth inhibitory activity via the microplate alamar blue (MABA) and low oxygen recovery (LORA) assays $(47,74,134)$. Briefly, the MABA assay is a colorimetric assay that uses the color change of rezasurin to evaluate $M t b$ growth inhibitory activity under aerobic conditions. On the other hand, the LORA assay evaluates potency against non-replicating $M t b$ cells under low oxygen conditions using a luminescent reporter strain. Significantly, in all cases the 
MIC values obtained from the LORA assays are lower than those from the MABA assays. To the best of our knowledge, it is the first observations that the molecules killed non-replicating $M t b$ at concentration below the MIC against $M t b$ grown under aerobic conditions; in all cases the values of $\mathrm{MIC}_{\mathrm{LORA}} / \mathrm{MIC}_{\mathrm{MABA}}$ were $<1$ (Table 2-1). In the class A molecules $(R)-9$ was two-fold more potent than rac-9 and $(S)-9$ in the MABA assays. Similar to an observed trend in MenA enzyme inhibitory assays, the carbamate $(R)$-19 could improve over 5 -fold increase in mycobactericidal activity compared to rac9. $(R)-19$ exhibited a significant activity in the LORA assay; the MIC value of $(R)-19$ is 1.7-fold more effective in killing non-replicating bacteria than rifampicin (MIC LORA 1.47 $\mu \mathrm{g} / \mathrm{mL})$. It is worth mentioning that $(R)-19$ was the most active in killing non-replicating $M t b$ in vitro among antimycobacterial drugs (approved by FDA) and preclinical drugs tested in our laboratory. Regardless of the stereochemistry of the chiral centers, the molecules in class B, C, and E did not show noticeable difference in antimycobacterial activity in the both MABA and LORA assays. The molecules in class D are regioisomers at the benzophenone moiety of the class A molecules. Similar to the molecules in class A, pronounced effect of the stereochemistry of secondary alcohol on antimycobacterial activity was observed in the molecules in class D. Contrary to the chirality effect observed in the molecules in class A, the molecules possessing $S$-configuration (in class D) exhibited better antimycobacterial activity than the corresponding $R$-configuration molecules. The effect of carbamate group on enzyme and bacterial growth inhibitory activities was not observed in the molecules in class D; the MABA MIC value of the carbamate $(S)$-23 was equal to the corresponding alcohol $(S)$-24. Nonetheless, the MABA MIC for the rac-alcohol 12 could be improved two-fold by the formation of carbamate; the MABA MICs of $r a c-12$ and $r a c-23$ were 6.25 and $3.25 \mu \mathrm{g} / \mathrm{mL}$, respectively. Antimycobacterial activity was improved by increasing the hydrophobicity of the side chain (C8 vs. C6) in the molecules of class A, whereas noticeable bactericidal effect by increasing hydrophobicity of the side chain was not observed in the molecules of class D. All molecules summarized in Figure 2-5 did not exhibit bactericidal activity against $S$. aureus at $60 \mu \mathrm{g} / \mathrm{mL}$ concentrations; lack of anti-staphylococcal activity of these molecules was well-correlated with their $S$. aureus MenA enzyme inhibitory activity $\left(\mathrm{IC}_{50}>60 \mu \mathrm{M}\right.$ against $S$. aureus $\mathrm{MenA}$ ).

\section{E. coli Growth Inhibitory Assays under Anaerobic Conditions}

In aerobic conditions, $E$. coli uses ubiquinone in its ETS. However, menaquinone is the lipoquinone used in anaerobic growth conditions. E. coli grown in anaerobic conditions may be used as a convenient surrogate for $M t b$ for initial screening of menaquinone inhibitors. We evaluated several known inhibitors of different targets for their effects on $E$. coli under anaerobic conditions, including electron transport system and menaquinone biosynthesis (Table 2-2). Only menA and electron transport inhibitors killed E. coli under anaerobic conditions. Figure 2-6A summarizes the effectiveness of inhibitors of different targets against $E$. coli under anaerobic conditions. Evaluation of gram negative bacterial inhibitors is a challenge due to efflux pumps. Bacterial efflux pumps are known to be active in anaerobic conditions, therefore an efflux pump inhibitor, 
Table 2-1. Antibacterial activities against $M t b / S$. aureus, and MenA enzyme inhibitory activities of all selected molecules.

\begin{tabular}{|c|c|c|c|c|c|c|}
\hline \multirow{2}{*}{ Compound } & \multicolumn{3}{|c|}{$\operatorname{MIC}(\mu \mathrm{g} / \mathrm{mL})$} & \multicolumn{3}{|c|}{ MenA Inhibition $(\mu \mathrm{M})$} \\
\hline & S.aureus ${ }^{\mathrm{a}}$ & Mtb (MABA) & Mtb (LORA) & S.aureus ${ }^{b}$ & $M t b^{b}$ & $\mathrm{IC}_{50}$ \\
\hline \multicolumn{7}{|c|}{ ( } \\
\hline$r a c-9$ & $>60$ & 12.5 & 5.20 & - & + & 15.0 \\
\hline$(S)-9$ & $>60$ & 12.5 & 5.59 & - & + & 7.5 \\
\hline (R)-9 & $>60$ & 6.25 & 4.91 & - & + & 17.0 \\
\hline (S)-18 & $>60$ & 12.5 & 4.91 & - & + & 16.0 \\
\hline (R) -18 & $>60$ & 6.25 & 5.59 & - & + & 9.0 \\
\hline$(S)-19$ & $>60$ & 12.5 & 2.94 & - & + & 9.0 \\
\hline (R)-19 & $>60$ & 2.31 & 0.85 & - & + & 1.5 \\
\hline (S)-20 & $>60$ & 12.5 & 12.5 & - & + & 15.0 \\
\hline$(R)-20$ & $>60$ & 2.31 & 0.85 & - & + & 1.2 \\
\hline \multicolumn{7}{|l|}{ Class B } \\
\hline$r a c-10$ & $>60$ & 12.5 & 1.88 & - & + & 15.0 \\
\hline$(S)-\mathbf{1 0}$ & $>60$ & 12.5 & 1.88 & - & + & 25.0 \\
\hline$(R)-10$ & $>60$ & 12.5 & 1.46 & - & + & 20.0 \\
\hline (S)-21 & $>60$ & 12.5 & 4.93 & - & + & 20.0 \\
\hline (R)-21 & $>60$ & 12.5 & 4.93 & - & + & 15.0 \\
\hline (R) -22 & $>60$ & 6.25 & 1.43 & - & + & 15.0 \\
\hline \multicolumn{7}{|l|}{ Class C } \\
\hline$r a c-\mathbf{1 1}$ & $>60$ & 12.5 & 3.00 & - & + & 15.0 \\
\hline$(S)-\mathbf{1 1}$ & $>60$ & 12.5 & 3.00 & - & + & 17.0 \\
\hline (R)-11 & $>60$ & 12.5 & 3.00 & - & + & 14.0 \\
\hline \multicolumn{7}{|l|}{ Class D } \\
\hline$r a c-12$ & $>60$ & 6.25 & 2.82 & - & + & 15.0 \\
\hline$r a c-23$ & $>60$ & 3.25 & 2.83 & - & + & 7.5 \\
\hline$(S)-12$ & $>60$ & 1.50 & 1.43 & - & + & 4.5 \\
\hline (R)-12 & $>60$ & 6.25 & 2.85 & - & + & 7.5 \\
\hline (S) -23 & $>60$ & 1.50 & 1.43 & - & + & 3.5 \\
\hline (R) -23 & $>60$ & 6.25 & 5.20 & - & + & 9.5 \\
\hline (S)-24 & $>60$ & 1.50 & 1.45 & - & + & 1.5 \\
\hline (S)-25 & $>60$ & 1.50 & 1.40 & - & + & 1.5 \\
\hline \multicolumn{7}{|l|}{ Class E } \\
\hline$r a c-13$ & $>60$ & 12.5 & 1.88 & - & + & 20.0 \\
\hline (S)-13 & $>60$ & 12.5 & 1.88 & - & + & 15.0 \\
\hline (R)-13 & $>60$ & 12.5 & 1.46 & - & + & 15.0 \\
\hline (S)-26 & $>60$ & 12.5 & 2.93 & - & + & 20.0 \\
\hline (R)-26 & $>60$ & 12.5 & 4.93 & - & + & 20.0 \\
\hline (S)-27 & $>60$ & 12.5 & 5.54 & - & + & 20.0 \\
\hline (R) -27 & $>60$ & 12.5 & 5.54 & - & + & 15.0 \\
\hline RIF & & 0.2 & 1.47 & & & \\
\hline INH & & 0.1 & $>128$ & & & \\
\hline
\end{tabular}


Table 2-1. (Continued).

NOTE: a MIC determined by broth dilution method, ${ }^{\mathrm{b}}$ At $100 \mu \mathrm{M}$ concentration of the compounds, + (MenA inhibition), - (No MenA inhibition), MABA -Microplate alamar blue assay, LORA -Low oxygen recovery assay, RIF- Rifampicin, INH - Isoniazid. 
Table 2-2. Activity of compounds against $E$. coli under anaerobic conditions.

\begin{tabular}{|c|c|c|c|}
\hline Target & Compounds & $\begin{array}{c}\text { Inhibition under } \\
\text { Anaerobic } \\
\text { Conditions }\end{array}$ & $\begin{array}{c}\text { Inhibition under } \\
\text { Aerobic } \\
\text { Conditons } \\
(\mu \mathrm{g} / \mathrm{mL})\end{array}$ \\
\hline \multirow[t]{4}{*}{ MenA } & AA-A, 7 & ++ & $>200$ \\
\hline & UT - $317((R)-20)$ & ++ & $>200$ \\
\hline & $\mathrm{UT}-281 \mathrm{R}$ & ++ & $>200$ \\
\hline & UT - 292R & ++ & $>200$ \\
\hline \multirow{8}{*}{ ETS } & Chlorpromazine & ++ & $60^{\mathrm{a}}$ \\
\hline & Pentachlorophenol & ++ & $20^{\mathrm{a}}$ \\
\hline & Methylene blue & ++ & $>200$ \\
\hline & Hydrazinecarboximidamide & ++ & $>200$ \\
\hline & Atovaquone & - & $>200$ \\
\hline & Trifluoperazine & - & 100 \\
\hline & Clofazimine & - & $>200$ \\
\hline & Resazurin & - & $>200$ \\
\hline \multirow[t]{11}{*}{ Protein synthesis } & Doxycycline & + & $1.5^{\mathrm{a}}$ \\
\hline & Pleuromutiline & + & 25 \\
\hline & Capreomycin & + & $>25$ \\
\hline & Chloramphenicol & + & $4^{\mathrm{a}}$ \\
\hline & Spectinomycin & + & 25 \\
\hline & Tobramycin & + & 4 \\
\hline & Amikacin & + & 4 \\
\hline & Erythromycin & - & $30^{\mathrm{a}}$ \\
\hline & Clindamycin & - & $12.5-50^{\mathrm{a}}$ \\
\hline & Linezolid & - & $>200$ \\
\hline & Kanamycin & - & 19 \\
\hline RNA polymerase & Rifampicin & + & 16 \\
\hline DNA Gyrase & Ciprofloxacin & + & 5 \\
\hline \multirow{2}{*}{$\begin{array}{l}\text { Folic acid } \\
\text { biosynthesis }\end{array}$} & Trimethoprim & + & $>200$ \\
\hline & Sulfamethoxazole & + & $>200$ \\
\hline Efflux pumps & Verapamil & + & $>200$ \\
\hline Cell permeability & Gramicidine & - & $>200$ \\
\hline \multirow[t]{3}{*}{ PG biosynthesis } & Penicillin G & - & $64^{\mathrm{a}}$ \\
\hline & Cephalosporin C & - & $64^{\mathrm{a}}$ \\
\hline & Vancomycin & - & $>200$ \\
\hline $\begin{array}{l}\text { Vitamin } \mathrm{K} \\
\text { analog }\end{array}$ & Warfarin & - & $>200$ \\
\hline $\begin{array}{l}\text { Membrane } \\
\text { perturbing }\end{array}$ & Polymyxin B nonapeptide & - & $64^{\mathrm{a}}$ \\
\hline
\end{tabular}

NOTE: +++ Bactericidal $(<25 \mu \mathrm{g} / \mathrm{mL})$, + Bacteriostatic, - No inhibition at $200 \mu \mathrm{g} / \mathrm{mL}$, ${ }^{a}$ Reported MIC values. 
A. E.coli growth inhibition under anaerobic conditions by the target
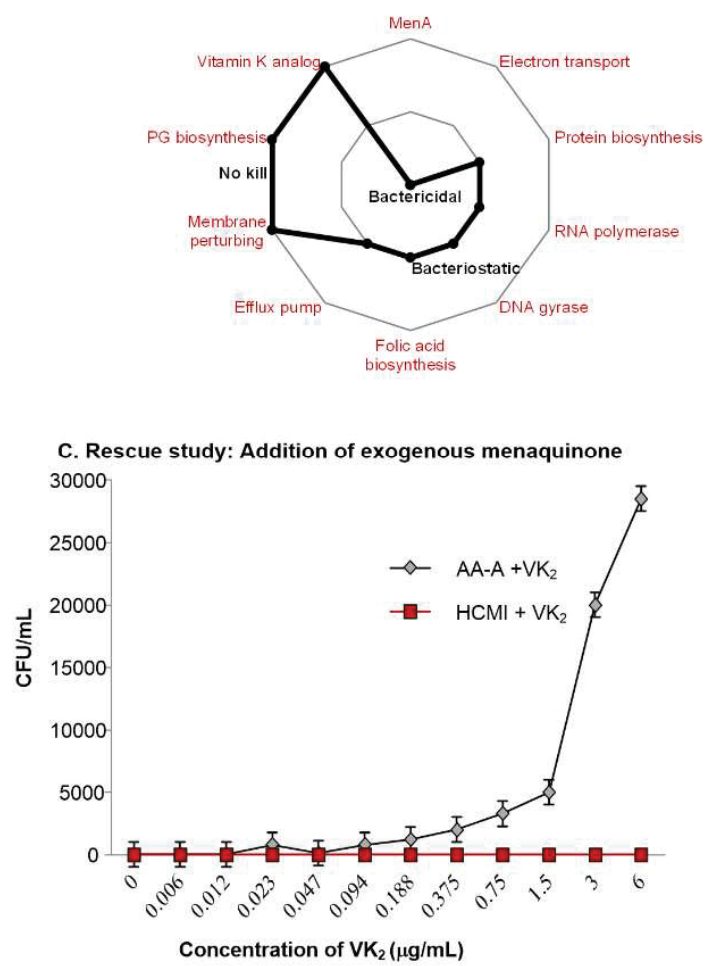
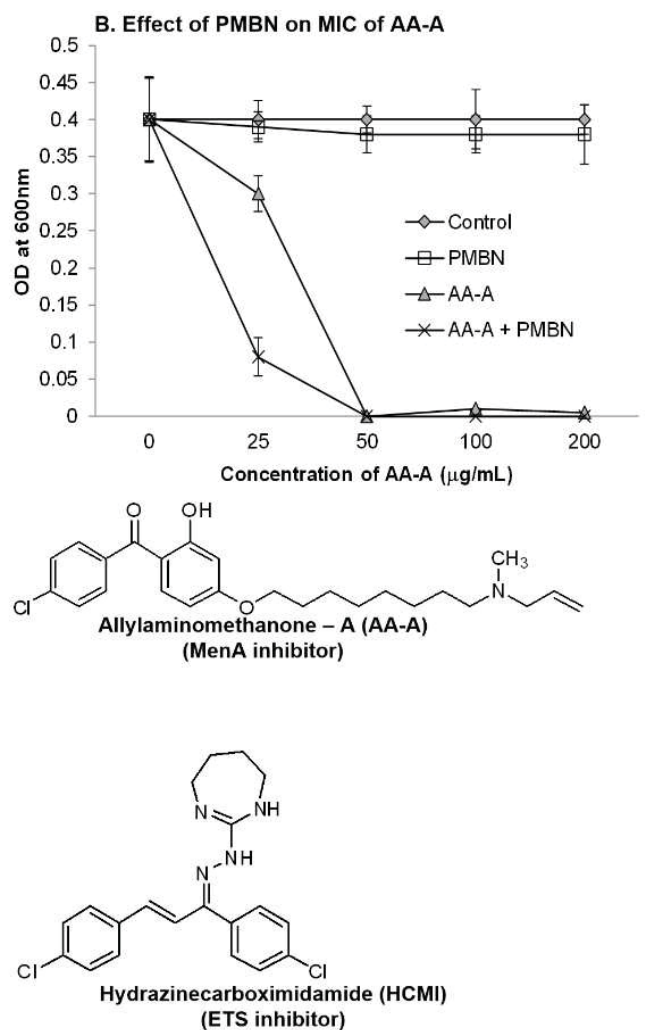

Figure 2-6. $\quad E$. coli growth inhibitory assays under anaerobic conditions.

A) Summary of bactericidal and bacteriostatic effect of compounds against $E$. coli under anaerobic conditions by target. B) Effect of polymyxin B nonapeptide (PMBN) on MIC of allylaminomethanone-A (AA-A, 7) in inhibition of $E$. coli under anaerobic conditions. Amount of PMBN added was $25 \mu \mathrm{g} / \mathrm{mL}$. C) Rescue study - Exogeneous menaquinone was added in varying concentrations to evaluate if the $E$. coli cells under anaerobic conditions can be rescued from the effect of AA-A and HCMI. 
verapamil was supplied in the assay along with inhibitor molecules to reduce the efflux of inhibitor molecules. Additionally, we supplemented membrane perturbing agent, polymyxin B nonapeptide (PMBN) to improve permeation of compounds into cells. In anaerobic conditions, verapamil did not show a major effect on the MICs of inhibitor compounds while supplementation of PMBN $(25 \mu \mathrm{g} / \mathrm{mL})$ was able to reduce the MIC of AA-A, 7 from $50 \mu \mathrm{g} / \mathrm{mL}$ to $25 \mu \mathrm{g} / \mathrm{mL}$ (Figure 2-6B). AA-A and other MenA inhibitors were ineffective against $E$. coli under aerobic conditions but displayed different effectiveness profile by being bactericidal under anaerobic conditions.

Our group has previously demonstrated that $M t b$ or $S$. aureus treated with the MenA inhibitors could not be rescued completely even at higher concentrations of exogenous menaquinone $\left(\mathrm{MK} / \mathrm{VK}_{2}\right)$. In contrast, growth inhibition of $E$. coli by the MenA inhibitor could be rescued by supplementation of $\mathrm{VK}_{2}(6 \mu \mathrm{g} / \mathrm{mL})$ under anaerobic conditions. E. coli growth inhibition rescued by addition of $\mathrm{VK}_{2}$ may be attributed to the degree of permeability of $\mathrm{VK}_{2}$ through cell envelope. Although lack of activity of MenA inhibitors against Gram-negative bacteria grown under aerobic conditions have been demonstrated, all MenA inhibitors identified in this program showed growth inhibition of E. coli at $5 \sim 10 \mu \mathrm{g} / \mathrm{mL}$ concentrations under anaerobic conditions, and the inhibitory effect of MenA inhibitor was rescued by supplementation of $\mathrm{VK}_{2}$ (Figure 2-6C). Therefore, these convenient cell-based assays using E. coli under anaerobic conditions can be utilized to confirm that the inhibitor molecules kill Gram-positive bacteria including $M t b$ by targeting MenA biosynthesis or bacterial electron transport systems.

\section{Oxygen Consumption Assays}

The basic concept underlying bacterial oxygen consumption assay is that changes in the rate of oxygen uptake result in a change in the oxygen concentrations. The oxygen consumption by bacterial concentrations greater than $10^{8} \mathrm{cfu} / \mathrm{mL}$ bacteria is proportional to the concentration of bacteria. Effect of the inhibitor molecule on electron transport by the quantitation of decolorization of methylene blue, which is a well-known redox dye, has been unambiguously demonstrated. Oxygen-consumption assays of $(R)-\mathbf{1 9}$ and $(S)-\mathbf{2 4}$ showed decolorization of methylene blue at concentrations $(12.5 \mu \mathrm{g} / \mathrm{mL})$ above the MIC of each molecule (MICs 2.31 and $1.50 \mu \mathrm{g} / \mathrm{mL}$, respectively) against $M t b$. Thus, we have concluded that $(R)-\mathbf{1 9}$ and $(R)-\mathbf{2 4}$ are very weak (or are not) electron transport system inhibitors, and thus, antimycobacterial activity of these molecules are attributed by targeting menaquinone biosynthesis.

\section{Cytotoxicity of MenA Inhibitors}

In order to obtain insight into potential toxicity of identified inhibitor molecules, all antimycobacterial MenA inhibitors were evaluated in in vitro cytotoxicity assays using Vero monkey kidney cells and $\mathrm{HepG}_{2}$ human hepatoblastoma cells . Most MenA inhibitors possessing the benzophenone group showed $\mathrm{IC}_{50}$ of $1-6.5 \mu \mathrm{g} / \mathrm{mL}$ against two mammalian cell lines; selectivity indexes ( $\mathrm{IC}_{50}$ in mammalian cells/MIC against $\mathrm{Mtb}$ ) of 
a series of benzophenone MenA inhibitors identified in this program were less than 2.49 On the other hand, O-methyl oxime derivatives in class A showed approximately 10-fold less cytotoxic than the corresponding benzophenone derivatives in in vitro cytotoxicity assays (Figure 2-7); $(R)-20$ showed an encouraging in vitro activity/toxicity ratio (a SI value $>10)$. It is believed that electrophilicity of the benzopheneone moiety of $(R)-\mathbf{1 9}$ can be diminished by O-methyl oxime formation of the benzopheneone. Thus, the benzophenone O-methyl oxime may not be a good electron acceptor that interferes with redox systems of mammalian cells.

\section{Antibacterial Activities of $(R)-19$ and $(R)-20$ against Drug-resistant $M t b$ and Other Mycobacterium Species}

Two MenA inhibitors molecules $(R)-\mathbf{1 9}$ and $(R)-20$ showed MIC values of 2.31 $\mu \mathrm{g} / \mathrm{mL}$ against $M t b\left(\mathrm{H}_{37} \mathrm{Rv}\right.$, a common laboratory strain). We resynthesized $(R)-19$ and $(R)-20$ and determined MICs against several other Mycobacterium species and drugresistant $M t b$. As summarized in Table 2-3, $(R)-\mathbf{1 9}$ and $(R)-\mathbf{2 0}$ showed mycobactericidal activity against rifampicin- and isoniazid-resistant strains at slightly lower concentrations (entries 2 and 3). (R)-19 and (R)-20 also killed other Mycobacterium species such as $M$. bovis, $M$. intracellulare, and $M$. smegmatis at relatively low concentrations $(3.0 \sim 6.5$ $\mu \mathrm{g} / \mathrm{mL})$. Thus, we concluded that new MenA inhibitors identified in this program kills Mycobacterium species selectively and are especially effective in killing $M t b$ at low concentrations. MenA inhibitors $(R)-\mathbf{1 9}$ and $(R)-\mathbf{2 0}$ effectively inhibited growth of drugresistant Mycobacterium species, indicating that MenA is a valid drug target to develop new drugs for MDR-Mycobacterium tuberculosis.

\section{Conclusions}

We have identified novel MenA (1,4-dihydroxy-2-naphthoate prenyltransferase) biosynthesis inhibitors by assay guided inhibitor design. We screened aurachin RE analogs through a detailed assay strategy. The identified MenA inhibitors demonstrated either very weak (or no) inhibitory activities of bacterial electron transport systems. A series of MenA inhibitors identified in this program exhibited antimicrobial spectrum focused against $M t b$. Selective activity against $M t b$ is ideal in TB drug discovery due to the fact that TB chemotherapy requires long regimen, and broad-spectrum anti-TB agents may cause resistance to other bacteria during TB chemotherapy. The carbamates $(R)-19$ and $(R)$-20 in class A showed significant growth inhibitory activities against nonreplicating $M t b$ (MIC $\mathrm{LORA}_{\text {, }} 0.85 \mu \mathrm{g} / \mathrm{mL}$ ) with the $\mathrm{MIC}_{\mathrm{LORA}} / \mathrm{MIC}_{\mathrm{MABA}}$ value of 0.37 $\left(\mathrm{MIC}_{\mathrm{LORA}} / \mathrm{MIC}_{\mathrm{MABA}}=7.35\right.$ for rifampicin). The effectiveness against non-replicating $M t b$ was also confirmed via assays using a modified Wayne model and nutrient deficient Loebel model assays $(72,135,136)(137)$. Among antimycobacterial agents tested in our laboratories, the inhibitor $(R)-\mathbf{1 9}$ and $(R)-\mathbf{2 0}$ were the most active in killing nonreplicating $M t b$ in vitro. A convenient assay with an $E$. coli model under hypoxic conditions was established and was used to screen compounds against MenA. This model may be used as a convenient surrogate for Mtb hypoxia. 


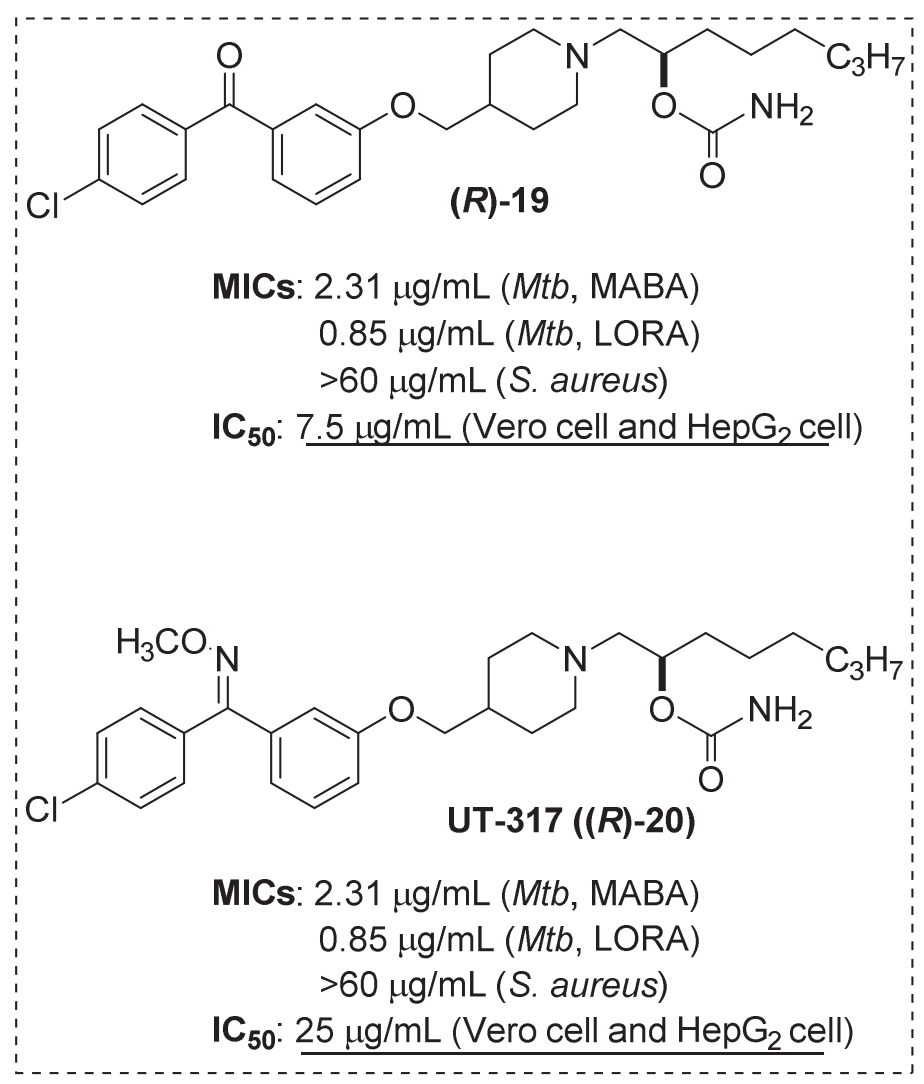

Figure 2-7. Optically active compounds with selective anti Mtb activity.

Table 2-3. MICs of selected compounds against Mycobacterium spp.

\begin{tabular}{|c|c|c|c|c|}
\hline \multirow{2}{*}{ Compounds } & \multicolumn{4}{|c|}{$\mathrm{MIC}^{\mathrm{a}}$} \\
\hline & $(R)-19$ & $(R)-\mathbf{2 0}$ & RIF & INH \\
\hline$M t b \mathrm{H}_{37} \mathrm{Rv}$ & 2.31 & 2.31 & 0.07 & 0.24 \\
\hline$M t b \mathrm{H}_{37} \mathrm{Rv} \mathrm{INHr}^{\mathrm{b}}$ & 2.91 & 1.52 & 0.03 & $>8$ \\
\hline$M t b \mathrm{H}_{37} \mathrm{Rv}_{\mathrm{RIFr}}{ }^{\mathrm{c}}$ & 2.91 & 1.51 & $>4$ & 0.05 \\
\hline M.bovis BCG & 3.00 & 2.95 & - & - \\
\hline M. intracellulare ATCC 15984 & 6.50 & 6.50 & - & - \\
\hline M. smegmatis ATCC 607 & 6.50 & 6.50 & - & - \\
\hline
\end{tabular}

NOTE: Mtb - Mycobacterium tuberculosis, MIC - Minimum inhibitory concentration, RIF - Rifampicin, INH - Isoniazid, ${ }^{a}$ MIC determined by broth dilution method (Microplate alamar blue method-MABA), ${ }^{\mathrm{b}} \mathrm{INH}$ resistant $M t b,{ }^{\mathrm{c}} \mathrm{RIF}$ resistant $M t b$. 
MenA inhibitors identified in this program strongly suggested that menaquinone biosynthesis is important in maintaining mycobacterial viability under conditions of restricted oxygen and nutrient stress (138). MenA inhibitors seem to be able to block the electron flow, consequently inhibiting the bacterial growth. It is conceptually very interesting that MenA inhibitors can be developed as indirect ATP synthesis inhibitors. The assay data for the identified MenA inhibitors indicate that menaquinone biosynthesis is a unique and new antimycobacterial target. In addition, in tuberculosis, the key to shortening the long regimen of TB treatment lies in targeting the non-replicating persistence subpopulation (vide supra). Therefore, the discovery of molecules that kill non-replicating $M t b$ at lower concentrations than MIC against $M t b$ under aerobic conditions is of great significance. Moreover, over the last several years, we are unable to isolate resistant bacteria to the MenA inhibitors in the mutation frequency studies.

Therefore, we concluded that unlike the other known bacterial molecular targets, menA shows extremely low mutation frequency.

In conclusion, robustness of our in vitro assay approaches to identify novel and selective MenA inhibitors against $M t b$ (summarized in Figure 2-3) has been demonstrated by the identification of a lead molecule $(R)-20$ (MIC LORA $_{\text {MIC }}$ MABA value of 0.37 , SI $>10)$. 


\section{CHAPTER 3. CELL WALL BIOSYNTHESIS INHIBITORS*}

\section{Introduction}

The cell wall of Mtb offers many unique targets for drug development. Mtb cell wall is implicated in the virulence and macrophage survival of Mtb $(101,139)$. Although, inhibitors of cell wall biosynthesis are included in TB treatment regimen, the significance of late stage cell wall biosynthesis as a drug development target against dormant $M t b$ is not well evaluated. There are reports of effectiveness of peptidoglycan biosynthesis inhibitors against non-replicating $M t b$. Meropenem (a carbapenem) was effective in killing non-replicating $M t b$ in combination with clavulanate (a $\beta$-lactamase inhibitor) (92). A novel series of cephalosporins and feropenem were also reported to be effective against non-replicating $M t b(93,140)$. Although, a mechanism of action of their bactericidal effect against dormant $M t b$ cells is not known, these are few examples that cell wall biosynthesis inhibitors kill dormant form of $M t b$. The mycolylarabinogalactan layers of the mycobacterial cell wall is significant for the dormant Mtb $(101,102)$. The first step in the mycolyl arabinogalactan biosynthesis in Mtb is catalyzed by prenylphosphate-GlcNAc-1-phosphate transferase (WecA). Inhibition of WecA could block the entire biosynthesis of essential cell wall components of Mtb in both replicating and nonreplicating states, and wecA enzyme could be a viable target for development of novel drugs. WecA and a peptidoglycan biosynthetic enzyme; MurX/MraY are members of P2HPT superfamily and are essential for Mtb growth (105). However, MurX/MraY inhibitors are effective in killing only replicating $M t b$ under aerobic conditions $(109,141$ 143). Although, WecA inhibitors have the potential to be effective TB drugs that kill nonreplicating $M t b$ under oxygen depleted conditions, only a few molecules are known to interfere with WecA and their effectiveness against non-replicating $M t b$ has been poorly characterized $(90,144)$. In order to establish the significance of WecA inhibition against the dormant $M t b$, it is crucial to evaluate selective WecA inhibitor molecules against dormant $M t b$.

* Adapted with permission.

- Siricilla, S., Mitachi, K., Skorupinska-Tudek, K., Swiezewska, E and Kurosu, M. (2014). Biosynthesis of a water-soluble lipid I analog and a convenient assay for translocase I. Anal. Biochem. 461, 36-45.

- Mitachi, K., Siricilla, S., Yang, D., Kong, Y., Skorupinska-Tudek, K., Swiezewska, E., Franzblau, S. G., and Kurosu, M. (2016). Fluorescence-based assay for polyprenyl phosphate-GlcNAc-1-phosphate transferase (WecA) and identification of novel antimycobacterial WecA inhibitors. Anal Biochem. 512, 78-90.

- Siricilla, S., Mitachi, K., Wan, B., Franzblau, S. G., and Kurosu, M. (2015). Discovery of a capuramycin analog that kills nonreplicating Mycobacterium tuberculosis and its synergistic effects with translocase I inhibitors. The J. Antibio. 68, 271-278 . 
Tunicamycin is a non-selective phosphotransferase inhibitor with both MurX and WecA inhibitory activity. Recently; Ishizaki et al. reported that caprazamycin (a MurX/MraY inhibitor) analog, CPZEN-45 inhibits mycobacterial WecA (90). Our group has reported novel muraymycin analogs (Muraymycin D1-amide and muraymycin D1diamide) with both MraY and WecA inhibition (144). Much studies are required to understand WecA in dormant Mtb. MurX inhibitor such as capuramycin has a faster killing profile towards replicating $M t b$, therefore, our strategy comprised of screening capuramycin analogs against MurX and WecA. A detailed screening for inhibitors of these enzymes requires a reliable and convenient assay. Therefore, we decided to develop convenient high throughput amenable assays to identify MurX and WecA inhibitor molecules routinely. The fluorescent probes, Park's nucleotide- $N^{\varepsilon}-\mathrm{C}_{6}$-FITC (32, for MraY/MurX) and UDP-glucosamine-C 6 -FITC (46, for WecA) are developed. These fluorescent probes enable us to screen the polyprenyl-phosphate $N$-acetylhexosamine 1phosphate transferase enzyme superfamily (e.g. MraY/MurX, WecA, AlgH, and DPAGT1) in high-throughput manner. A selective WecA inhibitor, UT-01320 (45) which kills non-replicating $M t b$ and intracellular $M t b$ was identified.

\section{Development of a Convenient MurX Assay}

Translocase I (MraY/MurX) catalyzes the transformation of UDP-MurNGlycpentapeptide and UDP-MurNAc-pentapeptide (Park's nucleotide) to the corresponding lipid I using decaprenyl $\left(\mathrm{C}_{50}\right)$ phosphate in Mycobacterium spp (Figure 3-1) $(145,146)$. This process is believed to be a reversible process in which E. coli MraY catalyzes an exchange reaction between UMP and lipid I to form Park's nucleotide in vitro (147). Lipid I isolated from $M t b$ contains the $\mathrm{C}_{50}$-prenyl unit that shows very poor watersolubility, and thus, this chemical characteristic of lipid I renders MurX enzyme assays impractical for screening.

Isolation and quantitation of Park's nucleotide and lipid I from in vitro MraY/MurX assay reaction mixtures are time-consuming processes (145). In addition, preparation of Mtb Park's nucleotide via semi-purified Mur enzymes is not amenable to multigram scale-up and the acquisition cost of enough decaprenyl phosphate for mediumto high-throughput screenings is very high. To date, several screening methods for MraY/MurX inhibitors have been reported that includes; 1) monitoring the transfer of phosphoryl-MurNAc-pentapeptide using fluorescent or radiolabeled Park's nucleotide and/or undecaprenyl phosphate (147), 2) measuring the exchange reaction between $\left[{ }^{3} \mathrm{H}\right]$ UMP to Park's nucleotide that requires separation of $\left[{ }^{3} \mathrm{H}\right]$ uridine after the treatment of alkaline phosphatase $(148,149), 3)$ an indirect assay using a coupled MraY-MurG that requires biotinylated Park's nucleotide and $\left.\left[{ }^{14} \mathrm{C}\right] \mathrm{UDP}-\mathrm{GlcNAc}(150), 4\right)$ an assay using HP20ss hydrophobic beads for isolating the generated radiolabeled lipid I (151), 5) a microplate-based assay using a radiolabeled-Park's nucleotide (152), and 6) a scintillation proximity assay using wheat germ agglutinin-coated beads to capture the lipid I from a radiolabeled-Park's nucleotide (153). Although a several assay methods were reported to be amenable to a HTS assay for MraY, in our hands, extraction of water- 


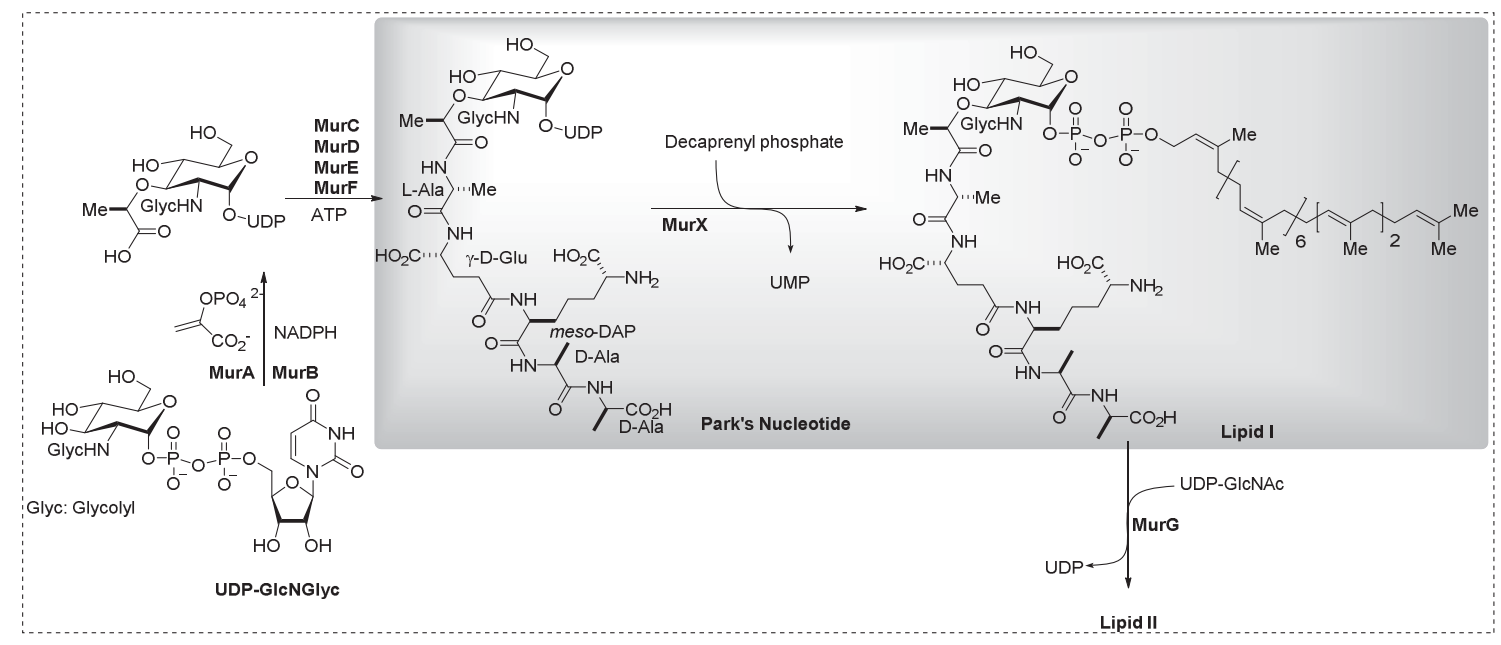

Figure 3-1. Biosynthesis of lipid II in Mtb.

Translocase I (MurX) catalyzes the transformation from Park's nucleotide to lipid I. 
insoluble lipid I derivative from assay media is essential $(147,153,154)$. In our attempt at developing reliable in vitro MraY/MurX assay, we concluded that the reported assays need further optimization to be robust statistical methods that can identify MraY/MurX inhibitors routinely with $\mathrm{IC}_{50}$ values. We established an efficient synthetic method for the generation of sufficient amount of fluorescent Park's nucleotide probes for HTS and tested the Park's nucleotide probes in MurX-catalyzed lipid I analog synthesis with decaprenyl and truncated prenyl phosphates $(155,156)$. Surprisingly, under the optimized conditions the water-soluble lipid I-neryl $\left(\mathrm{C}_{10}\right)$ analog could be biosynthesized efficiently with the Park's nucleotide probes and neryl phosphate (35).

Most of the reported MraY inhibitors were competitive to Park's nucleotide but not to prenyl-phosphate. Because capuramycin is a well-characterized MraY/MurX inhibitor that showed activities focused against Mycobacterium spp. (157), we selected positive and negative controls from capuramycin derivatives to develop a convenient MurX assay method, and the new MurX assay developed here was planned to demonstrate by screen antimycobacterial uridyl-peptide library molecules.

N-Glycolylated muramic (MurNGlyc) acid predominates in Mtb and MurNGlyc is the major muramyl building blocks in mycobacterial cell walls $(158,159)$. Thus, it is of importance to characterize specificity of MurX against N-glycolyl Park's nucleotide and $\mathrm{N}$-acetyl Park's nucleotide for discovery of novel antimycobacterial MurX inhibitors. In order to understand the tolerance of MurX in the structure of Park's nucleotide, we have synthesized a series of Park's nucleotide analogs according to our established chemoenzymatic or total chemical syntheses $(145,155,156)$. We and other groups have demonstrated that the dansyl- or FITC-conjugated N-acetyl Park's nucleotides can be applied to MraY enzyme assays with the MraY obtained from Gram-negative and some Gram-positive organisms (159). In our preliminary studies, the membrane fraction containing MraY or MurX enzyme (P-60) obtained from E. coli, S. aureus, $M$. smegmatis, or Mtb could transfer the fluorescent-Park's nucleotide conjugates to the corresponding undecaprenyl lipid I analogs with undecaprenyl phosphate in 5-25\% yields after $1 \mathrm{~h}$ incubation (160). These results were in accordance with the data previously reported by several other groups $(147,148)$. It was experimentally proven that MurX was also tolerated in the structure of the $\mathrm{C}_{6}$-position $\left(\mathrm{N}^{\varepsilon}\right)$ of lysine moiety of Park's nucleotides.

In order to further study tolerance of MurX against the structure of the N-acyl moiety of muramic acid in Park's nucleotide, we examined time-course experiments of lipid I syntheses with N-glycolyl Park's nucleotide- $\mathrm{N}^{\varepsilon}$-dansylthiourea and $\mathrm{N}$-acyl Park's nucleotide- $\mathrm{N}^{\varepsilon}$-dansylthiourea (Figure 3-2). MurX-catalyzed syntheses of lipid I analogs with 28 and 29 are summarized in Table 3-1. All reactions were performed in duplicate under the same reaction conditions except for the concentrations of decaprenyl phosphate and reaction time. The lipid I analogs (30 and 31 of Figure 3-2) were chemically synthesized as reference molecules for HPLC studies $(155,156)$. Direct comparison of the product yields for $\mathbf{3 0}$ and $\mathbf{3 1}$ revealed that Park's nucleotide-NE-dansylthiourea (28 and 29) were converted to the corresponding lipid I analogs 30 and 31, respectively, by MurX 


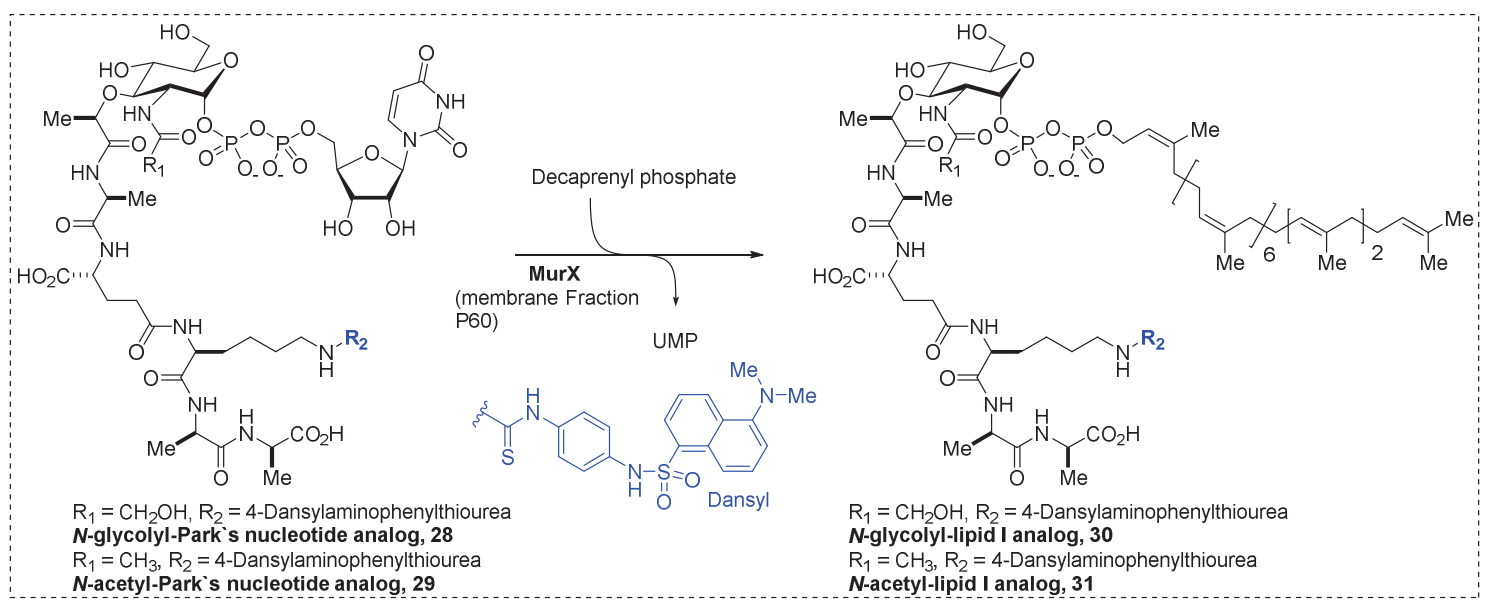

Figure 3-2. MurX-catalyzed syntheses of lipid I analogs from N-glycolyl- and Nacetyl-park's nucleotides.

Table 3-1. MurX-catalyzed syntheses of lipid I analogs from $N$-glycolyl- and $N$ acetyl-park's nucleotides.

\begin{tabular}{ccccc}
\hline Park's Nucleotide & $\begin{array}{c}\text { Decaprenyl Phosphate } \\
\text { (equivalents against 28 or 29) }\end{array}$ & $\begin{array}{c}\text { Lipid I } \\
\text { Reaction } \\
\text { time (h) }\end{array}$ & Yield (\%) \\
\hline $\mathbf{2 8}$ & 2 & $\mathbf{3 0}$ & 1 & $15-20$ \\
$\mathbf{2 8}$ & 2 & $\mathbf{3 0}$ & 3 & $15-20$ \\
$\mathbf{2 8}$ & 10 & $\mathbf{3 0}$ & 1 & $20-25$ \\
$\mathbf{2 9}$ & 2 & $\mathbf{3 1}$ & 1 & $15-20$ \\
$\mathbf{2 9}$ & 2 & $\mathbf{3 1}$ & 3 & $15-25$ \\
$\mathbf{2 9}$ & 10 & $\mathbf{3 1}$ & 1 & $20-25$ \\
\hline
\end{tabular}

NOTE: Reaction conditions - Park's nucleotide ( $2 \mathrm{mM} ; 3.75 \mu \mathrm{L}), \mathrm{MgCl}_{2}(0.5 \mathrm{M} ; 10 \mu \mathrm{L})$; $\mathrm{KCl}(2 \mathrm{M}, 10 \mu \mathrm{L})$, Triton X100 $(0.1 \% ; 11.25 \mu \mathrm{l})$, Tris-buffer $(\mathrm{pH}=8 ; 50 \mathrm{mM}, 5 \mu \mathrm{L})$, decaprenyl phosphate $(10 \mathrm{mM}, 2$ or 10 equivalents against 28 or 29), P-60 (15 $\mu \mathrm{L}), 26$ ${ }^{\circ} \mathrm{C}, 1$ or $3 \mathrm{~h},{ }^{\mathrm{a}} \mathrm{n}$-butanol extract was analyzed by HPLC (column: Kinetex 5u C8 100A, $150 \times 4.60 \mathrm{~mm}$, solvent $-\mathrm{CH}_{3} \mathrm{CN}: 0.05 \mathrm{M}$ aq. $\mathrm{NH}_{4} \mathrm{HCO}_{3}$, flow rate $-0.5 \mathrm{~mL} / \mathrm{min}$. 
without noticeable difference in reaction rate and product yield (Table 3-1). Increasing concentration of decaprenyl phosphate (from 2 to 10 equivalents against 28 or 29) did not dramatically increase the product yield (Table 3-1). Kinetic studies revealed that Nglycolyl Park's nucleotide- $\mathrm{N}^{\varepsilon}$-dansylthiourea and $\mathrm{N}$-acyl Park's nucleotide- $\mathrm{N}^{\varepsilon}$ dansylthiourea have a similar binding affinity towards MurX with the $\mathrm{K}_{m}$ values of 18.05 and $17.95 \mu \mathrm{M}$, respectively (147). Although Mtb predominantly uses N-glycolyl Park's nucleotide for the biosynthesis of peptidoglycan through N-glycolyl lipid I, MurX recognizes N-acetyl Park's nucleotide equally (158). These structural tolerances of the Park's nucleotide binding domain of MurX implies the possibility of further simplification of Park's nucleotide for development of a convenient MurX/MraY assay.

Mycobacterium spp. uses decaprenyl phosphate $\left(\mathrm{C}_{50}-\mathrm{P}\right)$ for the biosynthesis of lipid I (161). Due to the fact that $\mathrm{C}_{50}$-lipid I does not dissolve in water media, thus, isolation and quantitation of the generated lipid I in the assay reaction mixtures are timeconsuming processes (vide supra). In our hands, it is extremely difficult to develop medium- and high-throughput screening (HTS) for MurX/MraY assay using decaprenyl phosphate. We accomplished a practical chemical synthesis of neryl-lipid I and its fluorescent probes, and characterized their physicochemical properties. Neryl-lipid I-N ${ }^{\varepsilon}-$ dansylthiourea (33 of Figure 3-3A) showed excellent water-solubility $(>50 \mu \mathrm{g} / \mathrm{mL}$ ) and 33 can readily be analyzed via reverse-phase HPLC without gradient elution method (retention time: $<9$ min.) (Figure 3-4). MurX-catalyzed lipid I analog synthesis with Park's nucleotide- $\mathrm{N}^{\varepsilon}$-dansylthiourea and neryl phosphate (5 equivalent against 29) in the presence of $0.5 \%$ of triton X100 furnished neryl-lipid I-N ${ }^{\varepsilon}$-dansylthiourea in 3-5\% yield after $1 \mathrm{~h}$ (157). Under the same reaction conditions, the yield of $\mathbf{3 3}$ was proportionated to the concentrations of neryl phosphate; the same reaction with 60 equivalents of neryl phosphate furnished 33 in greater than $50 \%$ yield in $1 \mathrm{~h}$ (Figure 3-3B). As summarized in Figure 3-3C, effect of a phase transfer catalyst, triton X100 was observed in the transformation to 33 (162); $0.5 \%$ of triton X100 was determined to be the ultimate phase transfer catalyst of concentration for biosynthesis of lipid I-N $\mathrm{N}^{\varepsilon}$-dansylthiourea analog (33 with 29) and neryl phosphate. In short reaction times, application of higher concentrations of MurX enzyme (P-60) dramatically increased the reaction rate in biosynthesis of the lipid I analog (Fig 3-3D). However, Park's nucleotide- ${ }^{\varepsilon_{-}}$ dansylthiourea could be transformed to the neryl lipid I analog in greater than $90 \%$ yield even at lower concentrations of P-60 after $12 \mathrm{~h}$ (Figure 3-3E). Among the other reaction parameters examined for MurX-catalyzed neryl-lipid I synthesis, the reaction temperatures did not noticeably affect the reaction rate within a temperature range between 22 and $37^{\circ} \mathrm{C}$. Thus, MurX assays can be performed conveniently at the ambient air temperatures. It is worth mentioning that all data obtained with P-60 membrane fraction from $M t b$ could be reproduced with that from $M$. smegmatis.

\section{MurX/MraY Assays}

The MraY-catalyzed transformation of lipid I from Park's nucleotide is believed to be a reversible process $(147,163)$. On the contrary, under the optimized conditions 


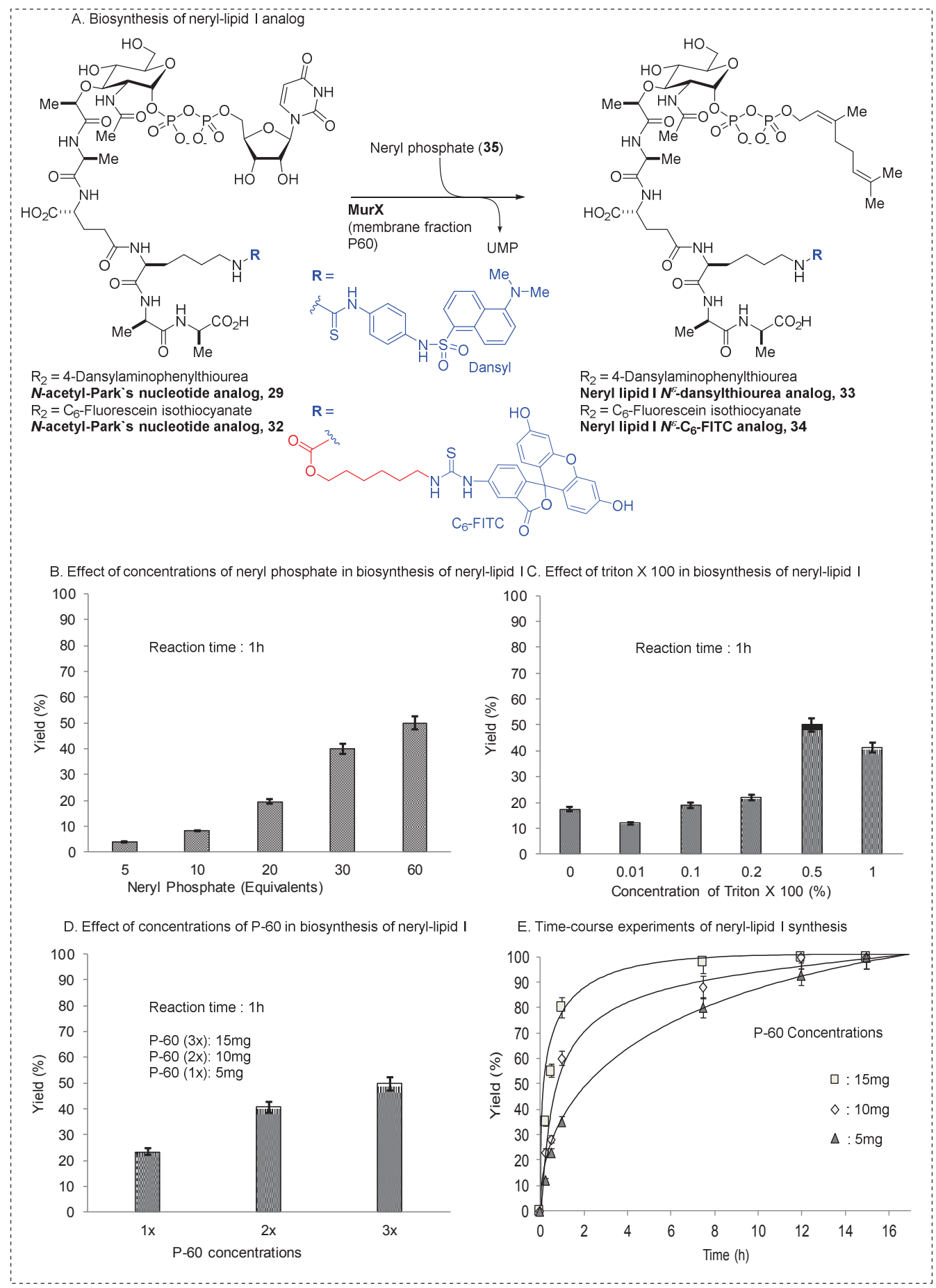

Figure 3-3. MurX-catalyzed biosynthesis of neryl lipid I analog.

A) Biosynthesis of neryl lipid I analog 33 from park's nucleotide analog 29 and neryl phosphate (35). B) Effect of concentrations of neryl phosphate in biosynthesis of 33. C) Effect of a phase-transfer catalyst, triton x100 in biosynthesis of 33. D) Effect of concentrations of MurX-containing membrane fraction (P-60). E) Time-course experiments of neryl-lipid I synthesis. 

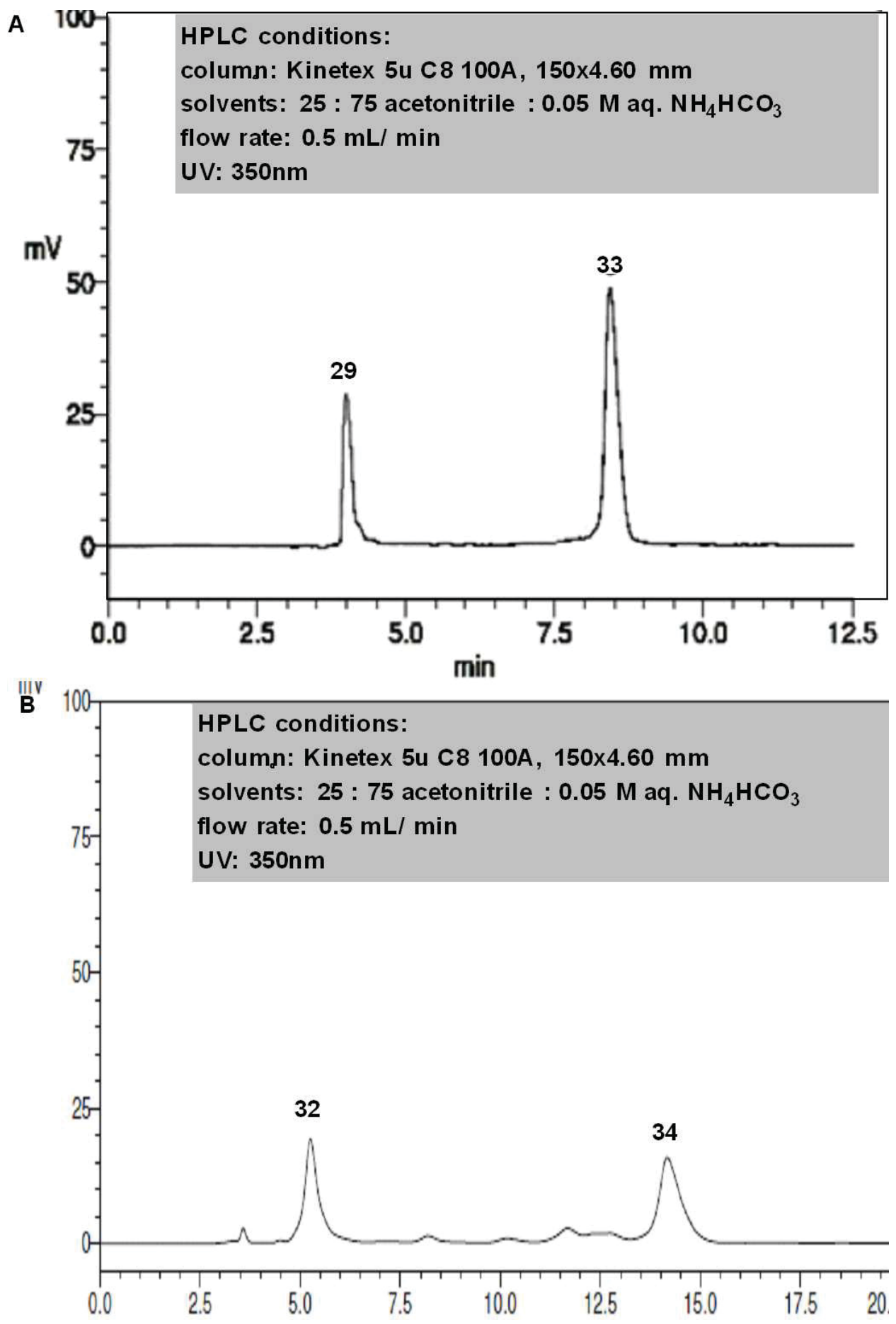

Figure 3-4. HPLC chromatogram of park's nucleotide- ${ }^{\varepsilon}$-fluorescent analogs (29 and 32 ) and neryl lipid $I-N^{\varepsilon}$-fluorescent analogs (33 and 34). 
MurX-catalyzed neryl-lipid I synthesis from Park's nucleotide- $\mathrm{N}^{\varepsilon}$-dansylthiourea is not an equilibrium reaction; $\mathbf{2 9}$ could be completely consumed to form $\mathbf{3 3}$ within $15 \mathrm{~h}$ (Figure 3-3E). The neryl-lipid I analog 33 can readily be dissolved in water or the assay media. Without extraction of the MurX/MraY enzymatic product from the reaction mixtures, the assay media can be assayed directly via reverse-phase HPLC for quantitation. Separation of Park's nucleotide analog 29 and neryl-lipid I analog 33 could be performed via a $\mathrm{C}_{8}$ or $\mathrm{C}_{18}$-reverse phase column with a fixed solvent system $\left(\mathrm{CH}_{3} \mathrm{CN}: 0.05 \mathrm{M}\right.$ aq. $\mathrm{NH}_{4} \mathrm{HCO}_{3}$ $=25: 75)$ at flow rate of $0.5 \mathrm{~mL} / \mathrm{min}$. Under these assay conditions, the retention times of Park's nucleotide 29 and neryl-lipid I 33 were $4.0 \mathrm{~min}$. and $7.9 \mathrm{~min}$, respectively (Figure 3-4). On the other hand, separation of Park's nucleotide and decaprenyl-lipid I required a gradient method for HPLC analyses (147); the retention time of decaprenyl-lipid I (31) was 60 min under our optimized HPLC conditions $\left(\mathrm{C}_{18}\right.$-reverse phase column, solvent systems: ( $\mathrm{MeOH}: 0.05 \mathrm{M}$ aq. $\mathrm{NH}_{4} \mathrm{HCO}_{3}=85: 15$ to $100 \% \mathrm{MeOH}$, flow rate: 2.0 $\mathrm{mL} / \mathrm{min}$ ). The $\mathrm{K}_{m}$ value for Park's nucleotide-dansylthiourea (29) was $18.29 \mu \mathrm{M}$ at the concentrations of $450 \mu \mathrm{M}$ of neryl phosphate; this was very similar to the $\mathrm{K}_{m}$ values obtained with decaprenyl or undeacprenyl phosphate $\left(\mathrm{K}_{m}: 18.05 \mu \mathrm{M}\right)(147)$. The $\mathrm{V}_{\max }$ for neryl-lipid I synthesis by MurX was determined to be $2.69 \times 10^{-3} \mu \mathrm{M} / \mathrm{sec}$ through the Michaelis-Menten plot. As stated above, significant difference in MurX/MraY-catalyzed neryl-lipid I synthesis is that the transformation from Park's nucleotide 29 to neryl-lipid I 33 is not a reverse process and neryl-lipid I 33 is biosynthesized in greater than $80 \%$ yield in $8 \mathrm{~h}$ (Figure 3-3E). Although the synthesis of neryl-lipid I 33 could be achieved with Park's nucleotide- $\mathrm{N}^{\varepsilon}$-dansylthiourea (29) in over $50 \%$ yield within $1 \mathrm{~h}$ via the purified MraY enzymes, unlike P-60 membrane fraction, the reactions did not attain over $65 \%$ yield even after $15 \mathrm{~h}$ due probably to instability of the purified MraY under the assay reaction conditions. Thus, MurX/MraY assays have been conveniently performed using P-60 membrane fractions obtained from Mtb or M. smegmatis.

The fluorescence characteristics of the $\mathrm{N}^{\varepsilon}$-dansylthiourea moiety in the enzymatic substrate and product can be applied to fluorescence-based analytical techniques in order to quantitate the inhibition of lipid I biosynthesis. The, signal-to-noise ratio of HPLC analyses using UV $(350 \mathrm{~nm})$ is excellent enough for quantitation of $10 \mu \mathrm{L}$ of the assay mixtures. We have also demonstrated that the FITC-conjugated N-acetyl Park's nucleotides (Park's nucleotide- ${ }^{\varepsilon}-\mathrm{C}_{6}$-FITC (32)) can be applied to MraY enzyme assays. Due to the wider applicability of fluorescein (FITC) for bioanalysis, $\mathbf{3 2}$ was utilized for the validation of MurX assay with antimycobacterial uridyl peptides. The range of linearity was established by injections (via an auto sampler) of six concentrations of Park's nucleotide-N ${ }^{\varepsilon}-\mathrm{C}_{6}$-FITC (32) and neryl-lipid I-N ${ }^{\varepsilon}-\mathrm{C}_{6}$-FITC (34) $(\mathrm{r} \geq 0.9)$, and limit of detection was determined to be much lower than $1 \mu \mathrm{M}$ concentrations.

\section{Validation of MurX Assays with Antimycobacterial Uridyl Peptides}

In order to determine usefulness of the MurX assay developed here, we examined several known MraY inhibitor molecules and negative controls, and demonstrated effectiveness of this MurX assay by screening our uridyl peptide library molecules (Figure 3-5). Known MraY inhibitors, capuramycin (36) and SQ641 (37) exhibited 


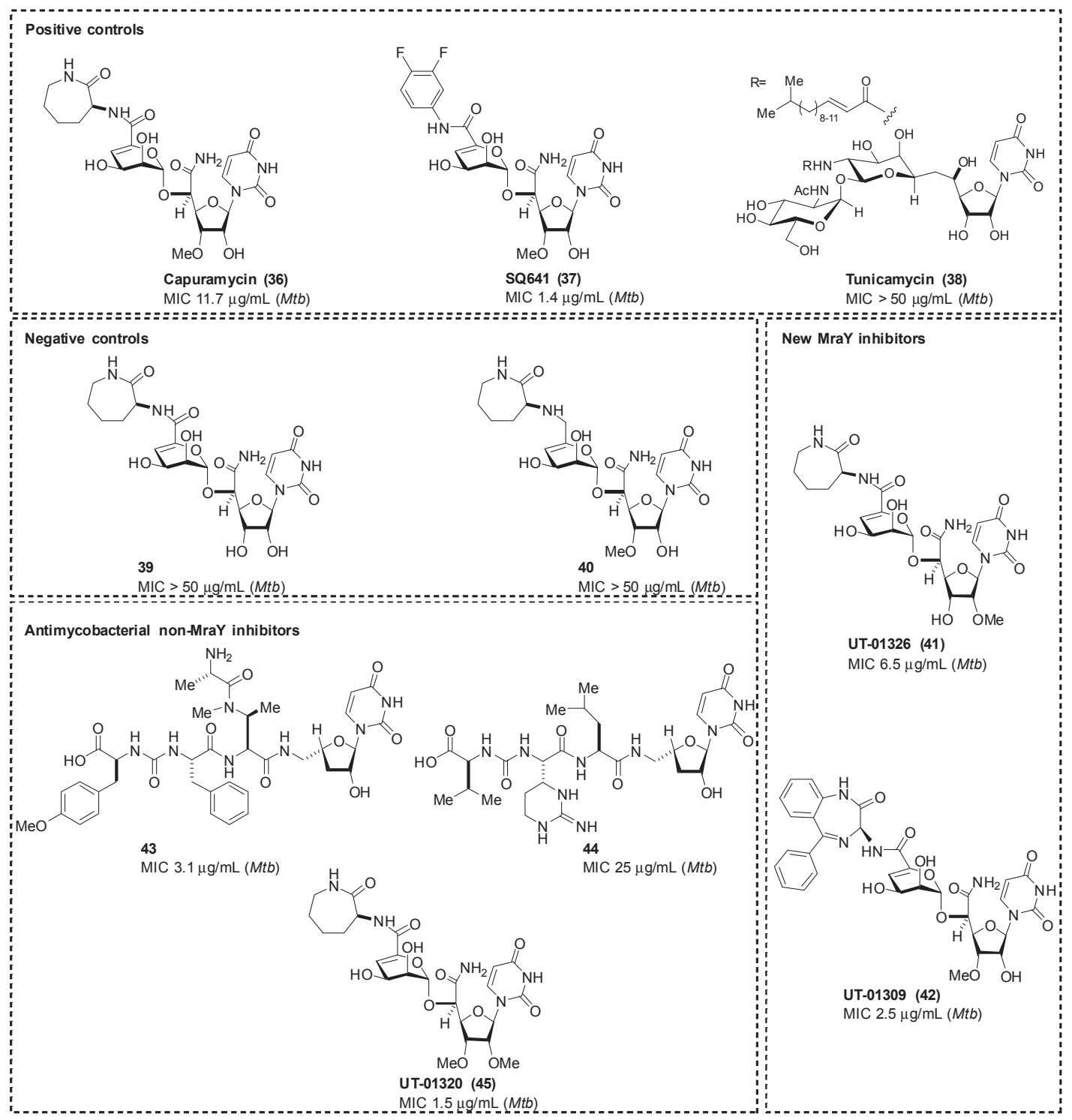

Figure 3-5. Library of uridyl peptides utilized for screening against MurX enzyme. 
strong enzyme inhibitory activity against MurX (entries 1 and 2 in Table 3-2); the $\mathrm{IC}_{50}$ values of capuramycin (36) and SQ641 (37) were 0.152 and $0.109 \mu \mathrm{M}$, respectively. Interestingly, these antimycobacterial MraY inhibitors 36 and $\mathbf{3 7}$ showed a 10-fold decrease in enzyme inhibitory activity against $E$. coli MraY. MurX enzyme inhibitory activity of tunicamycin (38) was determined to be the $\mathrm{IC}_{50}$ value of $2.73 \mu \mathrm{M}$ in which the observed activity of tunicamycin was closely related to the data reported in the literatures $(2.40-2.95 \mu \mathrm{M})(147)$. In our screening of series of MraY inhibitors in enzyme and bacterial growth inhibitory assays, the MurX inhibitors that showed the $\mathrm{IC}_{50}$ value of above $10 \mu \mathrm{M}$ did not exhibit significant bactericidal activity against $M t b$ (164-166). Thus, we set up an $\mathrm{IC}_{50}$ threshold of $10 \mu \mathrm{M}$ to distinguish exploitable antimycobacterial MurX inhibitors from other antimycobacterial molecules. The analogs of Capuramycin (36); 39 and 40 have been used as negative controls in our program. These molecules did not show MurX inhibitory activity even at $100 \mu \mathrm{M}$ concentrations (entries 4 and 5 in Table 3-2). Although vancomycin showed the good activity in MraY-MurG coupled assays, it could be confirmed that vancomycin did not inhibit MurX even at $100 \mu \mathrm{M}$ concentrations. We evaluated over 50 synthetic uridine-glycosyl peptides and uridyl peptides that showed the MIC values of $<25 \mu \mathrm{g} / \mathrm{mL}$ against Mtb in the MurX assay screening at three to five different concentrations. Among the identified new MurX inhibitors two molecules UT-01326 (41) and UT-01309 (42) are worthwhile highlighting. The 2'-methyl isomer 41 and amino-benzodiazepinone analog 42 showed increased MurX inhibitory activity compared to capuramycin (entry 7 and 8 in Table 3-2). On the other hand, the pacidamycin analog 43 and muraymycin analog 44 did not exhibit MurX inhibitory activity even at $100 \mu \mathrm{M}$ concentrations $(167,168)$. Thus, we concluded that dihydrouridyl analogs $\mathbf{4 3}$ and $\mathbf{4 4}$ exhibited antimycobacterial activity by targeting the other essential enzyme(s) for growth of Mtb. UT-01320 (45) (entry 11 in Table 3-2) showed no MurX inhibition and exhibited antimycobacterial activity.

Thus, the HPLC-based assay of MurX/MraY investigated here can be performed with standard analytical devises and will be adapted to medium- to high-throughput formats with close to ideal $Z^{\prime}$-factors (0.5-1.0) (169). The $Z^{\prime}$-factor was estimated from the data summarized in Table 3-2; an estimated $Z^{\prime}$-factor was 0.84, and thus, the new MurX/MraY assay method described here is considered to be an excellent assay. We are currently generating a relatively large number of library molecules containing known MurX/MraY inhibitors to examine robustness of the described assay method.

\section{Development of a Convenient WecA Transferase Assay}

WecA-catalyzed reactions have been performed via UDP- $\left[{ }^{14} \mathrm{C}\right] \mathrm{GlcNAc}, \mathrm{C}_{50}-\mathrm{P}$ (or $\left.\mathrm{C}_{55}-\mathrm{P}\right)$, and either purified WecA or crude membranes containing WecA. the reported assays require separation of the product by chromatography $(90,170-172)$. These assays are inadequate to systematically characterize library molecules in a high-throughput manner (104,151). We identified new UDP-GlcNAc fluorescent probes, UDPGlucosamine- $\mathrm{C}_{6}$-FITC and UDP-Glucosamine- $\mathrm{C}_{6}$-Dansyl, both of which can be recognized by the MurG transglycosylase, an essential peptidoglycan biosynthetic 
Table 3-2. Assay of positive- and negative-controls, and a library of uridyl peptides against MurX.

\begin{tabular}{|c|c|c|c|c|c|c|c|}
\hline \multirow{2}{*}{ Entry } & \multirow{2}{*}{ Compound } & \multicolumn{5}{|c|}{ MurX Inhibition (\%) ${ }^{\mathbf{a}}$} & \multirow{2}{*}{$\mathrm{IC}_{50}(\mu \mathrm{M})^{\mathrm{b}}$} \\
\hline & & $0.01 \mu \mathrm{M}$ & $0.1 \mu \mathrm{M}$ & $1 \mu \mathrm{M}$ & $10 \mu \mathrm{M}$ & $100 \mu \mathrm{M}$ & \\
\hline 1 & Capuramycin (36) & - & 36.6 & 88.4 & 100 & 100 & $0.152 \pm 0.0125(0.150-0.0180)^{\mathrm{c}}$ \\
\hline 2 & SQ 641 (37) & - & 40 & 95 & 100 & - & $0.109 \pm 0.00845(0.0150-0.100)^{\mathrm{c}}$ \\
\hline 3 & Tunicamycin (38) & - & 9 & 42 & 72 & 72 & $2.73 \pm 0.138(2.40-2.95)^{\mathrm{c}}$ \\
\hline 4 & 39 & - & 0 & 0 & 0 & 0 & ND \\
\hline 5 & 40 & - & 0 & 0 & 0 & 0 & ND \\
\hline 6 & Vancomycin & - & 0 & 0 & 0 & 0 & ND \\
\hline 7 & UT-01326 (41) & 3.8 & 81.2 & 100 & 100 & - & $0.105 \pm 0.00330$ \\
\hline 8 & UT-01309 (42) & - & 40 & 95 & 100 & - & $0.0950 \pm 0.00395$ \\
\hline 9 & 43 & - & 0 & 0 & 0 & 0 & ND \\
\hline 10 & 44 & - & 0 & 0 & 0 & 0 & ND \\
\hline 11 & UT-01320 (45) & 0 & 0 & 0 & 0 & 0 & ND \\
\hline 12 & DMSO & 0 & 0 & 0 & 0 & 0 & ND \\
\hline
\end{tabular}

NOTE: ${ }^{a}$ Reaction conditions - Park's nucleotide-N ${ }^{\varepsilon}-\mathrm{C}_{6}$-FITC (32) $(75 \mu \mathrm{M} ; 3.75 \mu \mathrm{L}), \mathrm{MgCl}_{2}(0.5 \mathrm{M} ; 10 \mu \mathrm{L}) ; \mathrm{KCl}(2 \mathrm{M}, 10 \mu \mathrm{L})$, Triton $\mathrm{X} 100(0.1 \% ; 11.25 \mu \mathrm{l})$, Tris-buffer $(\mathrm{pH}=8 ; 50 \mathrm{mM}, 2.5 \mu \mathrm{L})$, neryl phosphate $35(10 \mathrm{mM}, 45 \mu \mathrm{L})$, inhibitor molecule $(0-100 \mu \mathrm{M}$ in DMSO $(2.5 \mu \mathrm{L})), \mathrm{P}-60(15 \mu \mathrm{L}), 26^{\circ} \mathrm{C}, 3 \mathrm{~h}$, The $\mathrm{K}_{m} 18.29 \mu \mathrm{M}$ (Park's nucleotide 32$),{ }^{\mathrm{b}}$ The IC 50 values were obtained 3 times and the standard error of the mean was calculated, ${ }^{\mathrm{c}} \mathrm{The}_{\mathrm{IC}} \mathrm{C}_{50}$ values in parentheses were reported in the literatures see, references $(143,146,147,152,153)$ and/or were obtained via the assay conditions summarized in Table 3-1, The $\mathrm{K}_{m} 18.05 \mu \mathrm{M}$ (Park's nucleotide 32). 
enzyme $(173,174)$. Interestingly, under optimized conditions the water-insoluble decaprenyl-Glucosamine- $\mathrm{C}_{6}$-FITC and decaprenyl-Glucosamine- $\mathrm{C}_{6}$-Dansyl analogs could be biosynthesized with the WecA-containing membrane fractions obtained from Mtb, M. smegmatis, and E. coli.

Although extensive studies on assay developments of MurX/MraY-type phosphotransferases have been reported (141,150,152-154), there are a limited number of studies reported on practical WecA assays $(90,151,172,175)$. The reported WecA assay protocols are not amenable to HTS and they require radiolabeled UDP-GlcNAc and chromatographic separation of the product for quantitation. To date, WecA-catalyzed reactions have been carried out under a variety of biochemical conditions in which the effects of $\mathrm{pH}$, concentrations of divalent cation and detergent, and effects of additives (ATP, EDTA, etc) were evaluated. Moreover, specificity of WecA against the structure of polyprenyl phosphate was reported (105,176-181). After extensive optimization efforts, we could achieve the high-yielding WecA-catalyzed reactions with UDPGlucosamine- $\mathrm{C}_{6}$-FITC or UDP-Glucosamine- $\mathrm{C}_{6}$-Dansyl and exogenous decaprenyl phosphate $\left(\mathrm{C}_{50}-\mathrm{P}\right)$ that significantly increases reliability of WecA assays.

\section{Specificity of WecA against Nucleotide Substrate and Optimization of Reaction Conditions}

While developing high-throughput screens for GlcNAc transferase such as MurG, we identified that UDP-Glucosamine- $\mathrm{C}_{6}$-FITC is recognized by Mtb MurG to form the decaprenyl-P-P-GlcNAc-MurNAc-(pentapeptide) (lipid II) analog. We sought to explore whether these modified UDP-GlcNAc derivatives could be applied for WecA assays. To facilitate the screening for WecA inhibitors, specificity of WecA against UDPGlucosamine-C $\mathrm{C}_{6}$-FITC (46) and UDP-Glucosamine-C $\mathrm{C}_{6}$-Dansyl (47) was examined using the membrane fraction (P-60) prepared from $M$. smegmatis and exogenous decaprenylphosphate $\left(\mathrm{C}_{50}-\mathrm{P}\right)$. Because WecA and MurX/MraY use different UDP-2deoxy-2-N-acetyl-D-hexosamine donor substrates, we first applied the optimized reaction conditions established for MurX/MraY (50 mM MgCl $2,0.5 \%$ Triton-X, $50 \mathrm{mM}$ Tris- $\mathrm{HCl}$ buffer at $\mathrm{pH}$ 8.0) to the WecA-catalyzed transformation from 46 and 47 to $\mathrm{C}_{50}-\mathrm{P}-\mathrm{P}-$ Glucosamine- $\mathrm{C}_{6}$-FITC 48 (or $\mathrm{C}_{50}$-P-P-Glucosamine- $\mathrm{C}_{6}$-Dansyl (49)) (141). The results show that $\mathrm{P}-60$-catalyzed phosphotransfer reactions of 46 and 47 with $\mathrm{C}_{50}-\mathrm{P}$ resulted in the formation of 48 and 49 with 7.5-10\% yields in $2 \mathrm{~h}$. The generated WecA products 48

and 49 could be separated by partition with n-butanol (n-BuOH). In this process, majority of 46 and 47 remained in the water phase. The consumption of 46 and 47 (water phase) and generation of $\mathbf{4 8}$ and 49 (n-BuOH phase) could be monitored via reverse-phase HPLC (solvent: $\mathrm{CH}_{3} \mathrm{OH}: 0.05 \mathrm{M}$ aq. $\mathrm{NH}_{4} \mathrm{HCO}_{3}=40: 60$ to 80: 20, UV: 485 or $350 \mathrm{~nm}$, flow rate: $0.5 \mathrm{~mL} / \mathrm{min}$ ). Under these conditions, the retention times of UDPGlucosamine-C 6 -FITC (46) and $\mathrm{C}_{50}-\mathrm{P}-\mathrm{P}-$ Glucosamine-C 6 -FITC (48) were $11.6 \mathrm{~min}$ and 21.2 min, respectively (Figure 3-6). The HPLC retention times of $\mathbf{4 8}$ and $\mathbf{4 9}$ were identical to those of the chemically synthesized molecules. Because of wider applicability of fluorescein (FITC) for bioanalysis, we decided to utilize UDP-Glucosamine- ${ }_{6}$-FITC towards the development of a WecA assay. 


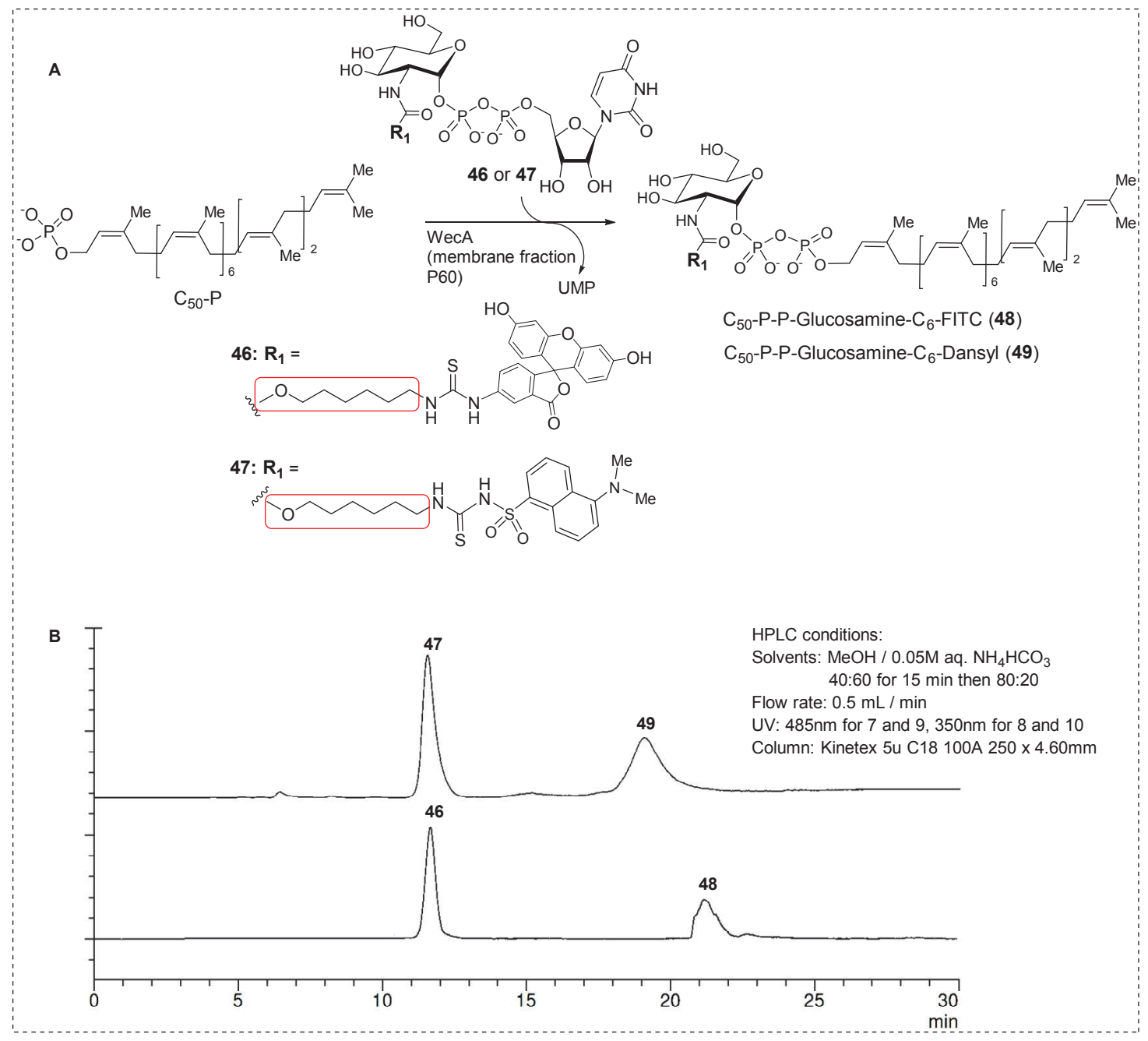

Figure 3-6. WecA-catalyzed biosynthesis of C50-P-P-Glucosamine-C6-fluorescent analogs 48 and 49.

A) UDP-GlcNAc donor analogs 46 and 47 can be recognized by WecA. B) Fluorescence detection-based HPLC at the endpoint of the reaction. 
Although the synthesis of $\mathrm{C}_{50}-\mathrm{P}-\mathrm{P}-\mathrm{Glucosamine}-\mathrm{C}_{6}-\mathrm{FITC}$ (48) could be achieved with $7.5-10 \%$ yield in $2 \mathrm{~h}$ using the UDP-GlcNAc fluorescent probe, $\mathrm{C}_{50}-\mathrm{P}$, and $\mathrm{P}-60$, optimization of reaction condition is important to improve assay efficiency and to reduce false-positive and false-negative errors in high-throughput screening (HTS) assays. The effects of adding ATP, EDTA, detergent, and co-factors have been previously reported for WecA-catalyzed reactions by using radiolabeled UDP-GlcNAc and undecaprenyl phosphate $\left(\mathrm{C}_{55}-\mathrm{P}\right)$. In general, conversion of over $20 \%$ or more of reactions provides reliable analyses in fluorescence-based assays for screening; enzyme inhibitory assays of conversion of $10 \%$ or below produce high standard errors by false-positive and falsenegative assay data.

WecA-catalyzed synthesis of $\mathrm{C}_{50}-\mathrm{P}-\mathrm{P}-\mathrm{Glucosamine}-\mathrm{C}_{6}$-FITC (48) with UDPGlucosamine- $\mathrm{C}_{6}$-FITC (46) and decaprenyl phosphate (3 equivalents against 46) in the presence of $0.5 \%$ of triton X (optimized concentrations for MurX/MraY) furnished 48 in 7.5-10\% yields after $2 \mathrm{~h}$. As summarized in Figure 3-7A, the effect of surfactant, CHAPS was observed in the transformation from 46 to 48 ; it was determined that $1 \%$ of CHAPS is the ultimate concentration of the surfactant $(103,181)$. Under the conditions with $1 \%$ of CHAPS, the optimum $\mathrm{MgCl}_{2}$ concentration was $40 \mathrm{mM}$ (Figure 3-7B); the same reaction furnished 9 in $46 \%$ yield at $37^{\circ} \mathrm{C}(\mathrm{pH} 8.0)$ after $2 \mathrm{~h}(103,172)$. In the timecourse experiments, application of higher concentrations of WecA enzyme (P-60) increased the product yield of $\mathbf{4 8}$. However, saturation of the product formation was observed at the concentration of P-60 at $15 \mu \mathrm{L}(1 \mathrm{mg} \mathrm{P}-60 / \mu \mathrm{L})$ (Figure 3-7C). Under the optimized conditions, the synthesis of $\mathbf{4 8}$ was increased in a time-dependent manner; WecA-catalyzed transformation of $\mathbf{4 6}$ with decaprenyl phosphate (3 equivalents) furnished 48 in 57.5\% yield after $6 \mathrm{~h}$ (Figure 3-7D). Among the other reaction parameters examined for $M$. smegmatis WecA-catalyzed synthesis of $\mathbf{4 8}$, the reaction temperatures $\left(30\right.$ vs. $\left.37^{\circ} \mathrm{C}\right)$ and $\mathrm{pH}(8.0$ vs. 8.3) did not noticeably affect the reaction rate. Although the other groups reported effects of ATP or EDTA in WecA-catalyzed GlcNAc-P-P-C 55 formation $(90,151,172)$, we concluded that these additives show no effect in the transformation from $\mathbf{4 6}$ to $\mathbf{4 8}$ with the P-60 obtained from M. smegmatis ( Figure 3-7E). It is worth mentioning that the active P-60 membrane consumed UDPGlucosamine- $\mathrm{C}_{6}$-FITC in the absence of exogenous $\mathrm{C}_{50}-\mathrm{P}$ but formed 46 by using endogenous polyprenyl phosphate(s). As illustrated in Figure 3-7F, the time-course study demonstrated that the consumption of $\mathbf{4 6}$ was well correlated to the generation of $\mathbf{4 8}$; in this reaction, 48 was formed in 5-10\% yield after $2-12 \mathrm{~h}$. The optimized conditions established with the P-60 membrane fraction from M. smegmatis were effective in the same reaction with the other sources of P-60 prepared from $M t b$ and E. coli. At the concentration of P-60 [15 $\mu \mathrm{L}(1 \mathrm{mg}$ P-60/ $\mu \mathrm{L})]$ optimized in Figure 3-7C, the P-60 prepared from a WecA overexpressed $E$. coli strain (E. coli $\mathrm{B} 21 \mathrm{wec} A)$ dramatically improved the transformation from $\mathbf{4 6}$ to $\mathbf{4 8}$ compared to that with the P-60 from a wild type E. coli. (Figure 3-7D). Quantitations of the remaining 46 and generated 48 in each reaction mixture were performed via reverse-HPLC. Signal-to-noise ratio of HPLC analyses using UV $(485 \mathrm{~nm})$ is excellent for quantitation of $25 \mu \mathrm{L}$ of the assay mixtures. The range of linearity was established by injections (via an auto sampler) of six concentrations of chemically synthesized 46 and $48(r \geq 0.9)$, and limit of detection was 

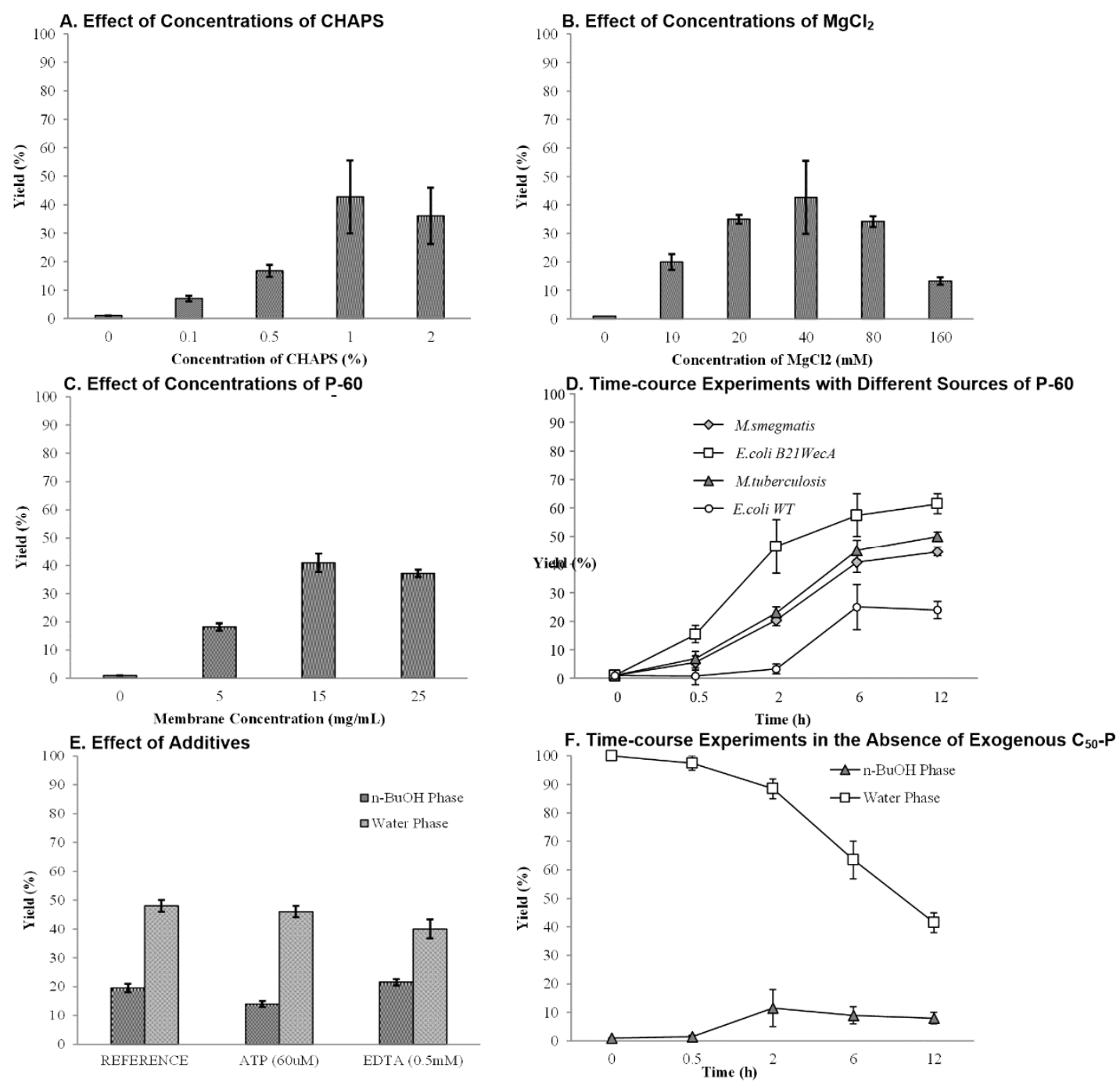

Figure 3-7. WecA-catalyzed biosynthesis of C50-P-P-Glucosamine-C6-FITC.

A) Effect of the phase transfer catalyst CHAPS. B) Effect of concentrations of $\mathrm{MgCl}_{2}$. C) Effect of concentrations of P-60 obtained from M. smegmatis. D) Time-course experiments of biosynthesis of $\mathbf{4 8}$ with different sources of membrane fractions. E) Effect of additives (ATP and EDTA). F) Time-course experiments of biosynthesis of $\mathbf{4 8}$ in the absence of exogenous $\mathrm{C}_{50}-\mathrm{P}$. 
determined to be much lower than $0.1 \mu \mathrm{M}$ concentrations. The $\mathrm{K}_{m}$ value for UDPGlucosamine- $\mathrm{C}_{6}$-FITC was $194.7 \mu \mathrm{M}$ at concentrations of $140 \mu \mathrm{M}$ of exogenous decaprenyl phosphate; this was similar to the $\mathrm{K}_{m}$ values obtained with undecaprenyl phosphate $\left(\mathrm{K}_{m}: 195.5 \mu \mathrm{M}\right)$. The $\mathrm{V}_{\max }$ for $\mathrm{C}_{50}$-P-P-Glucosamine- $\mathrm{C}_{6}$-FITC synthesis by the crude membrane containing $M$. smegmatis WecA (P-60) was determined to be $0.404 \mu \mathrm{M} / \mathrm{min}$ through the Michaelis-Menten plot. Significant difference in WecAcatalyzed phosphoryltransfer reactions is that the transformation between UDPGlucosamine- $\mathrm{C}_{6}$-FITC and $\mathrm{C}_{50}-\mathrm{P}-\mathrm{P}-\mathrm{Glucosamine}-\mathrm{C}_{6}$-FITC is not a reverse process, whereas the recent studies have concluded that Thermotoga maritima WecA-catalyzed reaction shows equilibrium between UDP-GlcNAc and $\mathrm{C}_{55}$-P-P-GlcNAc (182). Similarly, MraY/MurX-catalyzed phosphoryltransfer of polyprenyl phosphate to Park's nucleotide is known to be an equilibrium reaction (141).

\section{Development of UV/Vis Spectroscopy-based Assay for WecA}

$\mathrm{C}_{50}$-P-P-Glucosamine- $\mathrm{C}_{6}$-FITC is readily extracted with n-BuOH and UDPGlucosamine- $\mathrm{C}_{6}$-FITC remains in water media. Taking advantage of the hydrophobic character of $\mathbf{4 8}$, the fluorescence in $\mathrm{n}-\mathrm{BuOH}$ extract of WecA reaction was monitored via ultraviolet-visible (UV-vis) spectrometry (excitation of $485 \mathrm{~nm}$, emission of $528 \mathrm{~nm}$ ); the UV-vis-based assay was performed at a sufficiently high concentration of $\mathbf{4 6}$ $(22.5 \mu \mathrm{M})$ for $\mathrm{UV}-$ vis spectrometry and enough concentrations of $\mathrm{C}_{50}-\mathrm{P}$ that fulfill the $\mathrm{K}_{m}$ value. Progress of the WecA-catalyzed reaction of 46 was determined periodically and monitored for $12 \mathrm{~h}$. As shown in Figure 3-8B, an increase in fluorescence signal was observed in a time-dependent manner that was well-correlated to the yield curve obtained via the HPLC method (Figure 3-8A). A new assay method developed here was validated by demonstrating the inhibition of WecA activity by a known MurX/WecA inhibitor, tunicamycin; generation of $\mathbf{4 8}$ was inhibited by tunicamycin in a dose-dependent manner (Figure 3-8C). The $\mathrm{IC}_{50}$ value of tunicamycin against $M$. smegmatis WecA and E. coli WecA was determined to be $0.59 \mu \mathrm{g} / \mathrm{mL}$ and $0.12 \mu \mathrm{g} / \mathrm{mL}$ by constructing dose-response curves via UV-vis spectrometry (Figure 3-8B). Figure 3-9 shows WecA inhibitors identified in this assay. The data summarized in Table 3-3 were obtained from at least three independent experiments and the standard deviation was less than $10 \%$. It was determined that DMSO did not inhibit the WecA assays at 5\% (v/v) concentrations. However, inhibition of the reactions was started at 10\% (v/v) of DMSO; approximately $50 \%$ of the enzyme activity was reduced at this concentration (Figure 3-8D).

\section{Specificity of WecA against the Structures of Prenyl Phosphate}

We identified that neryl phosphate (cis- $\left.\mathrm{C}_{10}-\mathrm{P}\right)$ and $(2 \mathrm{Z}, 6 \mathrm{E})$-farnesyl phosphate (cis- $\mathrm{C}_{15}-\mathrm{P}$ ) could be recognized by the MurX/MraY-type phosphotransferases to form the corresponding lipid I analogs, whereas, trans $-\mathrm{C}_{10}-\mathrm{P}$ and $-\mathrm{C}_{15}-\mathrm{P}$, and cis- and trans-phytols $\left(\mathrm{C}_{20}-\mathrm{P}\right)$ were not recognized by MurX/MraY enzymes $(141,183)$. To investigate the specificity of WecA against truncated prenyl phosphates, we evaluated WecA-catalyzed 

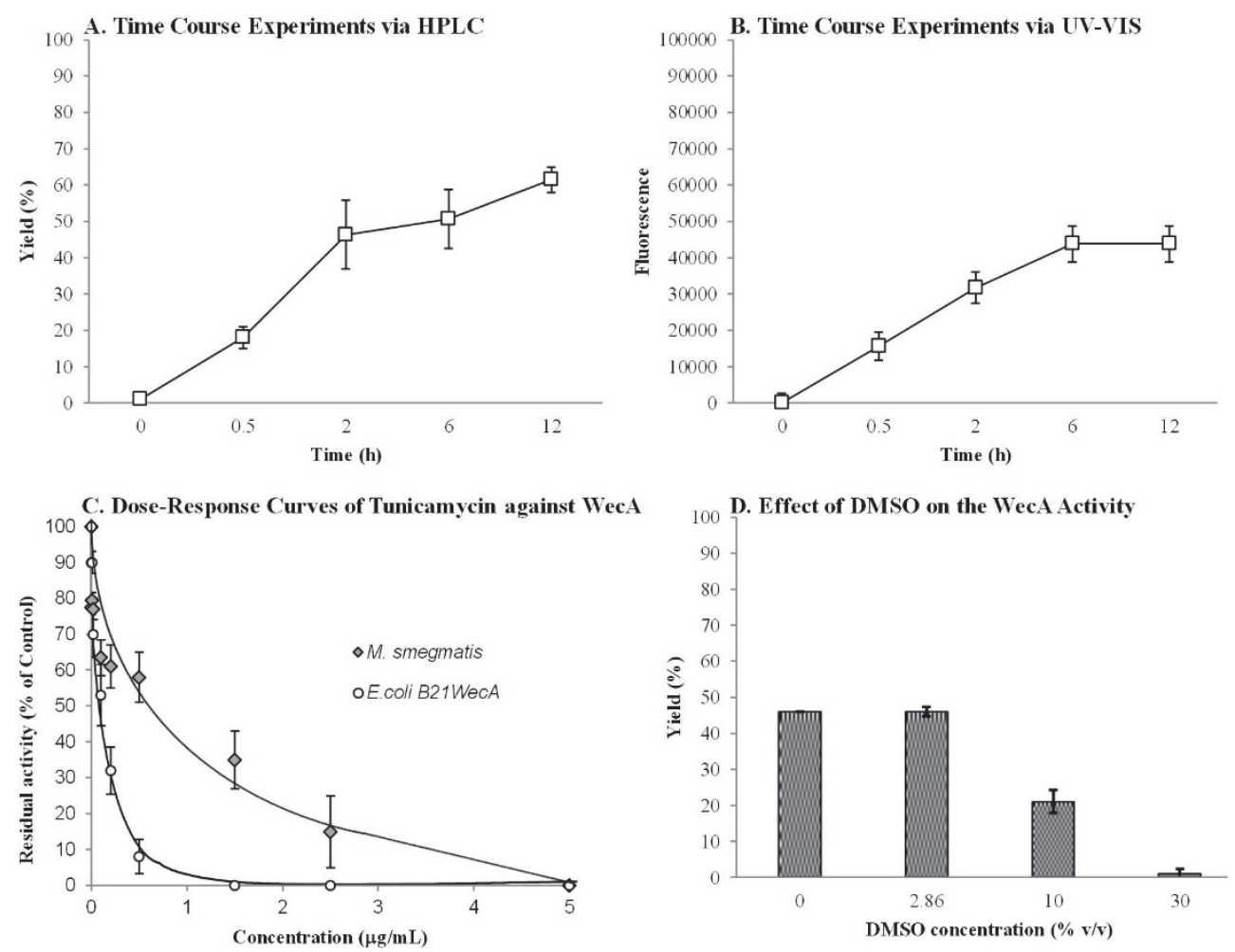

Figure 3-8. Validation of WecA assays via uv-vis.

A) Time-course experiments of biosynthesis of $\mathbf{4 8}$ monitored via HPLC. B) Time-course experiments of biosynthesis of $\mathbf{4 8}$ monitored via UV-vis. C) Dose-response curves of tunicamycin against E. coli and M. smegmatis WecA. D) Effect of DMSO on the WecA activity. 


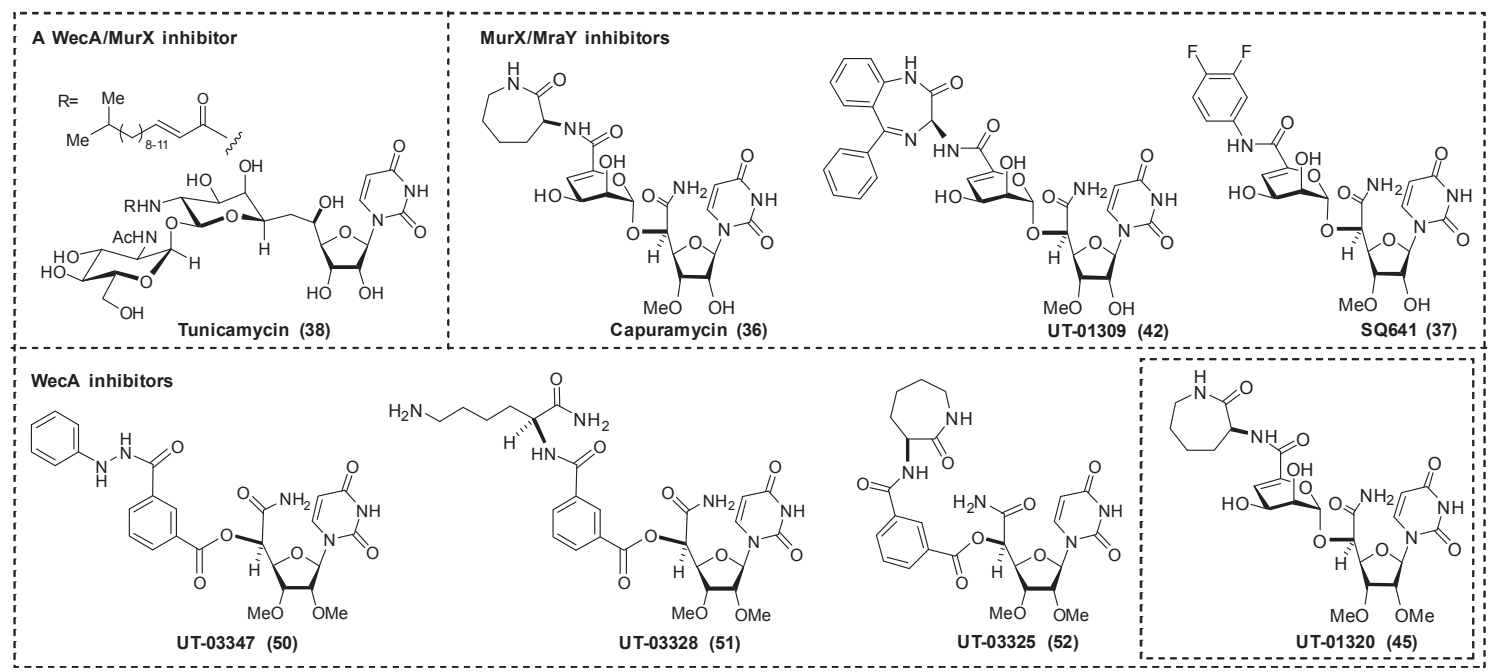

Figure 3-9. WecA inhibitors identified in this assay.

Table 3-3. Assays against a library of capuramycin analogs against the WecA enzyme (P-60 from $E$. coli B21 WecA).

\begin{tabular}{|c|c|c|c|c|}
\hline \multirow{2}{*}{ Compound } & \multicolumn{3}{|c|}{ WecA Inhibition (\%) } & \multirow{2}{*}{$\mathrm{IC}_{50}(\mu \mathrm{g} / \mathrm{mL})^{\mathrm{t}}$} \\
\hline & $0.02 \mu \mathrm{g} / \mathrm{mL}$ & $2 \mu \mathrm{g} / \mathrm{mL}$ & $20 \mu \mathrm{g} / \mathrm{mL}$ & \\
\hline Tunicamycin (38) & $36.0(38.5)^{\mathrm{c}}$ & $97.0(100)$ & $100(100)$ & $0.120 \pm 7.80$ \\
\hline Capuramycin (36) & - & - & - & - \\
\hline UT-01309 (42) & - & - & - & - \\
\hline SQ 641 (37) & - & - & - & - \\
\hline UT-03347 (50) & $11.0(15.5)$ & $23.0(29.0)$ & $80.5(84.5)$ & ND \\
\hline UT-03328 (51) & $0(0)$ & $23.0(28.0)$ & $60.5(75.5)$ & ND \\
\hline UT-03325 (52) & $30.0(35.0)$ & $35.0(45.0)$ & $55.0(60.5)$ & ND \\
\hline UT-01320 (45) & $42.0(45.0)$ & $100(100)$ & $100(100)$ & $0.0354 \pm 9.14$ \\
\hline DMSO & $0(0)$ & $0(0)$ & $0(0)$ & - \\
\hline
\end{tabular}

NOTE: ${ }^{a}$ Reaction conditions - UDP-Glucosamine-C6-FITC (46) (1, $\left.0.56 \mu \mathrm{L}\right), \mathrm{MgCl}_{2}(0.5$ $\mathrm{M} ; 4 \mu \mathrm{L})$, CHAPS $(5 \% ; 10 \mu \mathrm{l}), \beta$-mercaptoethanol $(50 \mathrm{mM}, 5 \mu \mathrm{L})$ Tris-buffer $(\mathrm{pH}=8.0$, $50 \mathrm{mM}, 9.74 \mu \mathrm{L}), \mathrm{C}_{50}-\mathrm{P}(4 \mathrm{mM}, 1.7 \mu \mathrm{L}), \mathrm{P}-60$ (E. coli B21WecA) $(18 \mu \mathrm{L}), 37^{\circ} \mathrm{C}, 2 \mathrm{~h}$, The $\mathrm{K}_{m}: 194.7 \mu \mathrm{M}$ (for 46 ), ${ }^{\mathrm{b}}$ Each experiment was performed three times, and the average $\mathrm{IC}_{50}$ values were summarized, ${ }^{\mathrm{c}} \mathrm{Wec} \mathrm{A}$ inhibitory activity in parentheses were obtained via HPLC, ND - Not determined. 
transformations of UDP-Glucosamine- $\mathrm{C}_{6}$-FITC with a series of synthetic prenyl phosphates. Due to the fact that endogenous decaprenyl phosphate $\left(\mathrm{C}_{50}-\mathrm{P}\right)$ that exists in the M. smegmatis P-60 membrane could be utilized in the WecA-catalyzed transformation of 48, generations of prenyl-P-P-Glucosamine- $\mathrm{C}_{6}$-FITC analogs with the truncated lipid-P were determined by comparison of the retention times of the chemically synthesized analogs via HPLC. The generated products were also quantitated by HPLC. Unlike MurX/MraY enzymes, $\mathrm{C}_{10}-\mathrm{P}, \mathrm{C}_{15}-\mathrm{P}$, and $\mathrm{C}_{20}-\mathrm{P}$ were not incorporated by WecA; the corresponding prenyl-P-P-Glucosamine- $\mathrm{C}_{6}$-FITC derivatives were not formed (Figure 3-10). In these experiments, $\mathrm{C}_{50}$-P-P-Glucosamine- $\mathrm{C}_{6}-\mathrm{FITC}$ was generated in 5$10 \%$ yield. Undecaprenyl phosphate $\left(\mathrm{C}_{55}-\mathrm{P}\right)$ was equally effective to $\mathrm{C}_{50}-\mathrm{P}$ in WecA catalyzed transformation from $\mathbf{4 6}$ to 48 using P-60 prepared from M. smegmatis.

\section{Screening a Library of Capuramycin-based Analogs via the New UV-VIS- based WecA Assay}

To identify WecA inhibitors, we applied the UV-vis-based WecA assay to screen an optimized library of 50 capuramycin analogs in duplicate with 384-well plates. In these screening, the P-60 membrane fraction isolated form E. coli B21WecA was applied. All compounds were screened at three different concentrations $(0.02,2.0$, and $20 \mu \mathrm{g} / \mathrm{mL})$. Each plate contained four control wells: the first one with the denatured P-60 (heated at $100{ }^{\circ} \mathrm{C}$ for $\left.5 \mathrm{~min}\right)$, the second one without P-60, the third one addition of $30 \%(\mathrm{v} / \mathrm{v})$ of DMSO and the forth one with tunicamycin at $20 \mu \mathrm{g} / \mathrm{mL}$. Under the assay conditions, four molecules were identified as WecA inhibitors (50, 51, 52 and 45). The identified WecA inhibitors were confirmed by the HPLC-based assays at $0.02,2.0$, and $20 \mu \mathrm{g} / \mathrm{mL}$ concentrations. A dose-response curve of UT-01320 (45) against WecA obtained via UV-vis spectrometry determined the $\mathrm{IC}_{50}$ value of UT-01320 (45) to be $35.4 \mathrm{ng} / \mathrm{mL}$ (Figure 3-11). The observed inhibition of the WecA enzyme by 45 is more potent than that of tunicamycin $\left(\mathrm{IC}_{50} 0.12 \mu \mathrm{g} / \mathrm{mL}\right.$ ). It is important to note that the inhibitory activities of WecA inhibitors identified using P-60 from E. coli B21wecA was well-correlated to those against the WecA containing membranes from M. smegmatis ATCC607 (Figure 3-8C).

\section{Mycobactericidal Activity of UT-01320}

A 2'-methylated capuramycin analog, UT-01320 (45) did not exhibit MurX enzyme inhibitory activity even at $400 \mu \mathrm{g} / \mathrm{mL}$ concentrations; however, it was noticed that UT-01320 inhibited WecA with the $\mathrm{IC}_{50}$ values of $35.4 \mathrm{ng} / \mathrm{mL}$ (E.coli B21WecA) and $50.7 \mathrm{ng} / \mathrm{mL}$ (M. smegmatis wecA), respectively $(103,141)$. While screening of new capuramycin analogs against replicating and nonreplicating (dormant) $M t b$, it was found that 45 killed both replicating and nonreplicating $M t b$ in MABA, LORA and LOEBEL assays, respectively (91). To the best of our knowledge, it is the first observation that a capuramycin analog exhibited bactericidal activity against nonreplicating $M t b$ at low concentrations. 

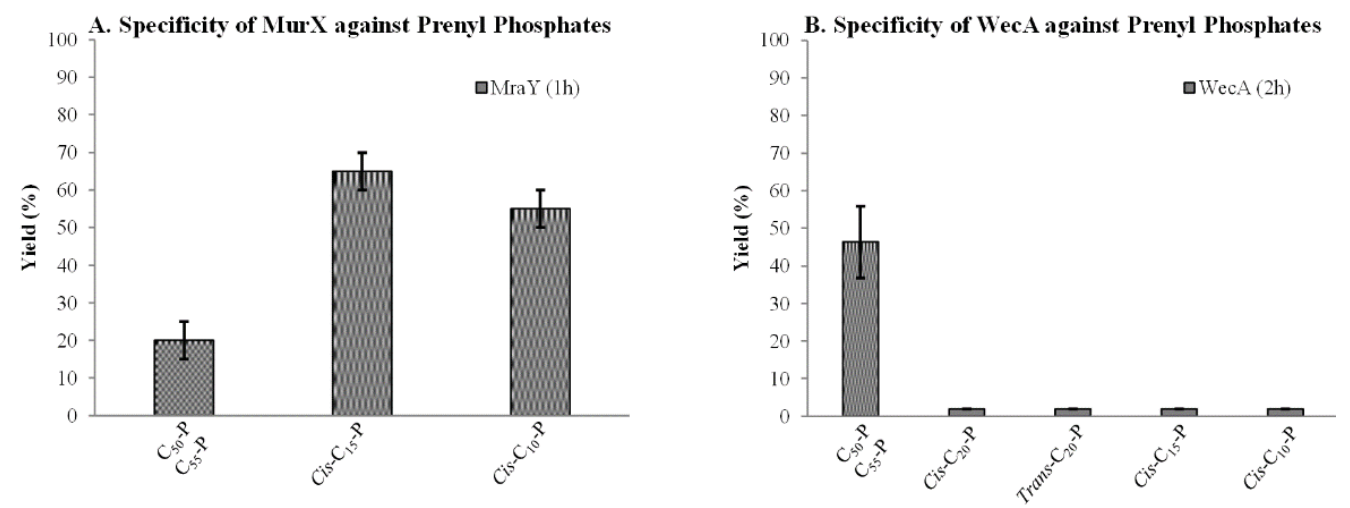

Figure 3-10. Comparison of specificity of MurX and WecA against prenyl phosphates.

A) Specificity of MurX against $\mathrm{C}_{55}-\mathrm{P}, \mathrm{C}_{50}-\mathrm{P}, c i s-\mathrm{C}_{15}-\mathrm{P}$ and $c i s-\mathrm{C}_{10}-\mathrm{P}$. B) Specificity of M. smegmatis WecA against $\mathrm{C}_{55}-\mathrm{P}, \mathrm{C}_{50}-\mathrm{P}$, cis- and trans- $\mathrm{C}_{20}-\mathrm{P}$, cis- $\mathrm{C}_{15}-\mathrm{P}$ and $c i s-\mathrm{C}_{10}-\mathrm{P}$.

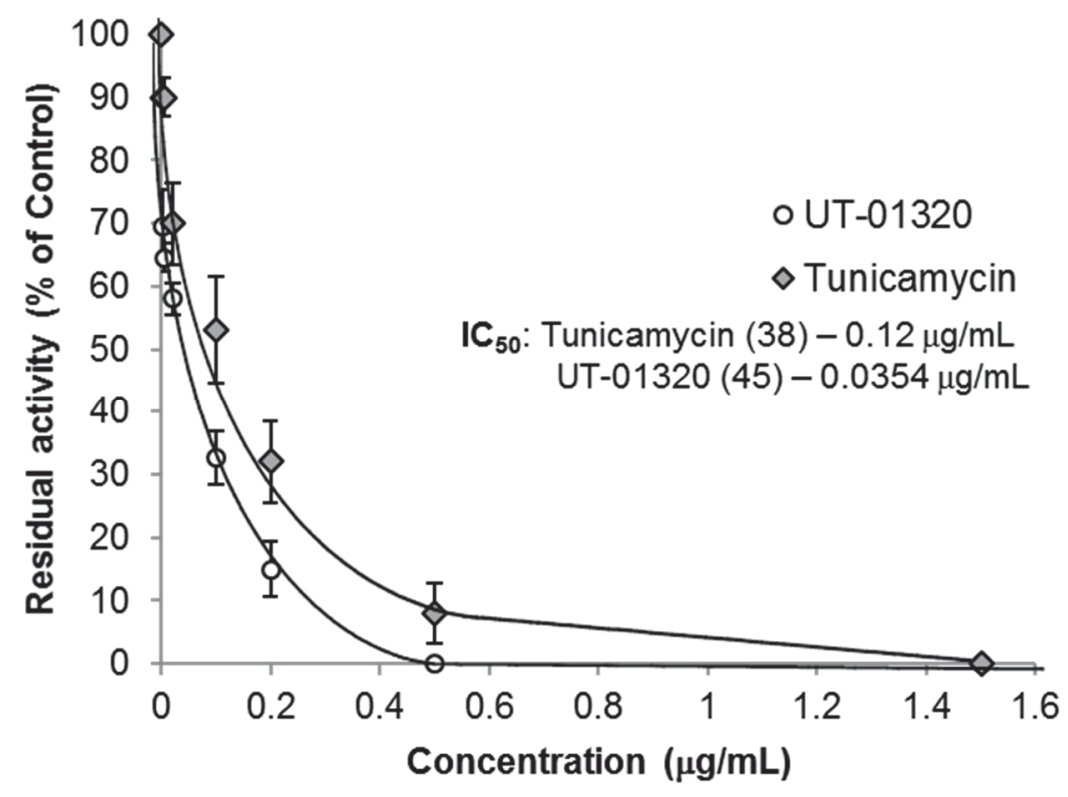

Figure 3-11. Dose-response curves for the WecA inhibitory activities of UT-01320 and tunicamycin.

WecA containing membrane fraction (P-60) was prepared from E. coli $\mathrm{B} 21 \mathrm{WecA}$ and applied to the inhibition studies. 
The novel MurX and WecA assays were useful in studying the structure-activity relationship of Capuramycin towards improving efficacy of its antimycobacterial activity in vitro and in vivo (143). Daiichi-Sankyo and Sequella reported several capuramycin analogs in which in vitro MraY enzyme and antimycobacterial activity could be improved via the modification of the carboxylic group of the capuramycin biosynthetic intermediate, A-500359 $(142,143,184,185)$. Our group has synthesized new capuramycin analogs via our total synthetic scheme, in which all analogs are structurally different from the reported molecules and they are difficult to access from A-500359 (164). To date, we have synthesized Capuramycin (36), SQ 641 (37), and over 100 new capuramycin analogs in which their structures were diversified with optically active amines (R3) and the four uridyl templates, 3'O-methyl (natural form), 2'O-methyl, 2',3'O-dimethyl and 3'O-demethylated (unnatural forms) uridine-mannopyranuronate derivatives (Figure 3-12). The syntheses of capuramycin analogs with these templates were accomplished in our lab via the synthetic scheme reported previously $(164,165)$. As the results of in vitro evaluation of these molecules in MurX enzyme inhibition and $M t b\left(\mathrm{H}_{37} \mathrm{Rv}\right)$ growth inhibitory activities against replicating and nonreplicating (dormant) Mtb via the MABA and LORA assays, it was identified that UT-01309 (42) possessing 3-amino-1,4benzodiazepine-2-one exhibited increased MurX enzyme and $M t b$ growth inhibitory activity (MIC: $2.5 \mu \mathrm{g} \mathrm{ml}^{-1}$ vs $12.0 \mu \mathrm{g} \mathrm{ml}^{-1}$ for capuramycin, IC $500.095 \mu \mathrm{M}$ vs $0.127 \mu \mathrm{M}$ for capuramycin) $(47,136,141,186)$. The analogs synthesized from the 3 'O-methyluridinemannopyranuronate (natural form) did not exhibit bactericidal activity against nonreplicating $M t b$ even at $400 \mu \mathrm{g} \mathrm{ml}^{-1}$ concentrations. The isocapuramycin (2'O-methyl) analogs $\left(\mathrm{R}_{1}=\mathrm{Me}\right.$ and $\mathrm{R}_{2}=\mathrm{H}$ in Figure 3-12) showed equal MurX inhibitory and antimycobacterial activities to the corresponding analogs derived from the natural form $\left(\mathrm{R}_{1}=\mathrm{H}\right.$ and $\mathrm{R}_{2}=\mathrm{Me}$ in Figure 3-12). The antimycobacterial MurX inhibitors, isocapuramycin analogs (2'O-methyluridine-mannopyranuronate derivatives) were not capable of killing nonreplicating $M t b$ in the LORA assay. The 3'O-demethyl capuramycin analogs $\left(\mathrm{R}_{1}, \mathrm{R}_{2}=\mathrm{H}\right.$ in Figure 3-12) did not exhibit activities in MurX enzyme inhibitory and antimycobacterial assays. Remarkably, 2'O-methylated capuramycin, UT-01320 (45) killed both replicating and nonreplicating $M t b$ at low concentrations with the

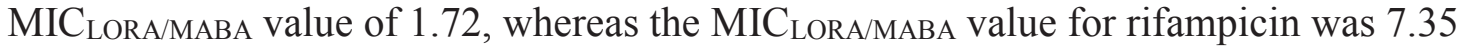
(Table 3-4). The MIC LORA/MABA value close to 1 or below 1 is considered to be ideal TB drug lead molecules in our programs. 45 was also tested against nutrient deficient model of $M t b$ (LOEBEL) (187). In both models of non-replicating Mtb, no CFU was observed after 15 days' incubation of the $M t b$ cultures treated with UT-01320 at $100 \mu \mathrm{g} \mathrm{ml}^{-1}$, $10 \mu \mathrm{g} \mathrm{ml}^{-1}$ and $2.58 \mu \mathrm{g} \mathrm{ml}^{-1}$, respectively (Figure 3-13). Cytotoxicity ( $\mathrm{IC}_{50}$ ) of 45 was evaluated in vitro using Vero monkey kidney cells and $\mathrm{HepG}_{2}$ human hepatoblastoma cells. As observed for other capuramycin analogs, UT-01320 did not show cytotoxicity even at $400 \mu \mathrm{g} \mathrm{ml}^{-1}$ concentrations (Table 3-4); the selectivity index ( $\mathrm{IC}_{50}$ in mammalian cells/MIC against $M t b$ ) of $\mathbf{4 5}$ was determined to be $>266$.

\section{Effectiveness of UT-01320 against Intracellular Mtb}

Activity of UT-01320 45 against intracellular $M t b$ was evaluated. Murine macrophage cell J774A.1 infected by a transformant Mtb CDC1551 containing tdTomato 


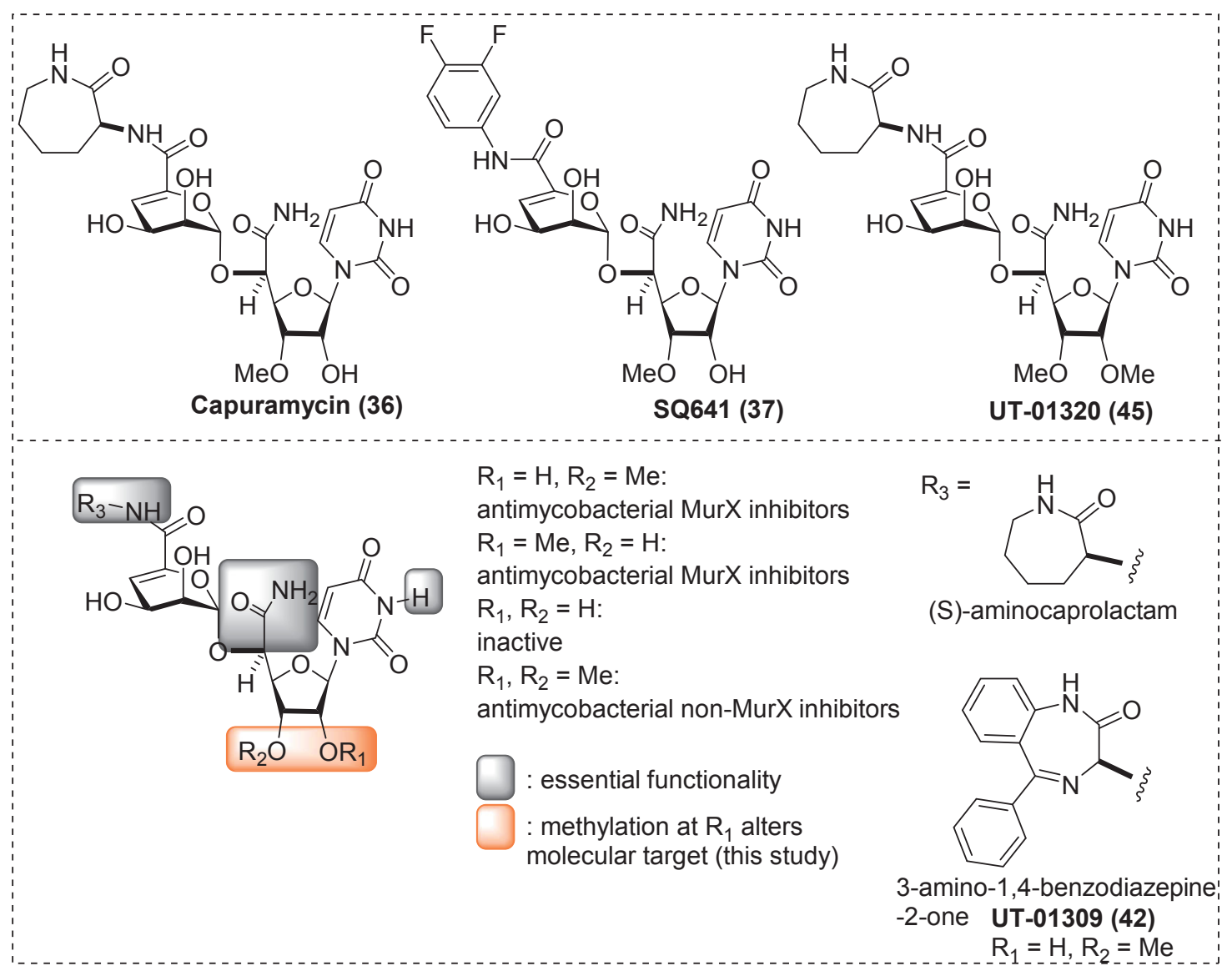

Figure 3-12. Structures of capuramycin (36), SQ 641 (37), UT-01320 (45), and UT01309 (42) and summary of SAR obtained from a 100-membered library. 
Table 3-4. MICs of selected compounds against Mycobacterium spp.

\begin{tabular}{lcccc}
\hline Compounds & $\begin{array}{c}\text { IC } 5 \text { MurX } \\
\boldsymbol{\mu M}\end{array}$ & $\begin{array}{c}\text { MIC (MABA) } \\
\boldsymbol{\mu g} / \mathbf{m L}\end{array}$ & $\begin{array}{c}\text { MIC (LORA) } \\
\boldsymbol{\mu g} / \mathbf{m L}\end{array}$ & $\begin{array}{c}\text { IC } \mathbf{5 0}(\text { Vero Cells) } \\
\boldsymbol{\mu g} / \mathbf{m L}^{\mathbf{a}}\end{array}$ \\
\hline Capuramycin & 0.127 & 12.5 & $>100$ & $>400$ \\
$(\mathbf{3 6})$ & & & & \\
SQ 641 (37) & 0.098 & 1.56 & $>100$ & $>400$ \\
UT-01320 (45) & $>100$ & 1.50 & 2.58 & $>400$ \\
UT-01309 (42) & 0.095 & 2.50 & $>100$ & $>400$ \\
RIF & - & 0.20 & 1.47 & $>400$ \\
INH & - & $0.04-0.10$ & $>100$ & $>400$ \\
EMB & - & 0.78 & $>100$ & $>400$ \\
\hline
\end{tabular}

NOTE: MABA - Microplate alamar blue assay, LORA - Low oxygen recovery assay, ${ }^{a}$ Cytotoxicity against Vero monkey kidney cells was $>400 \mu \mathrm{g} / \mathrm{mL}$.
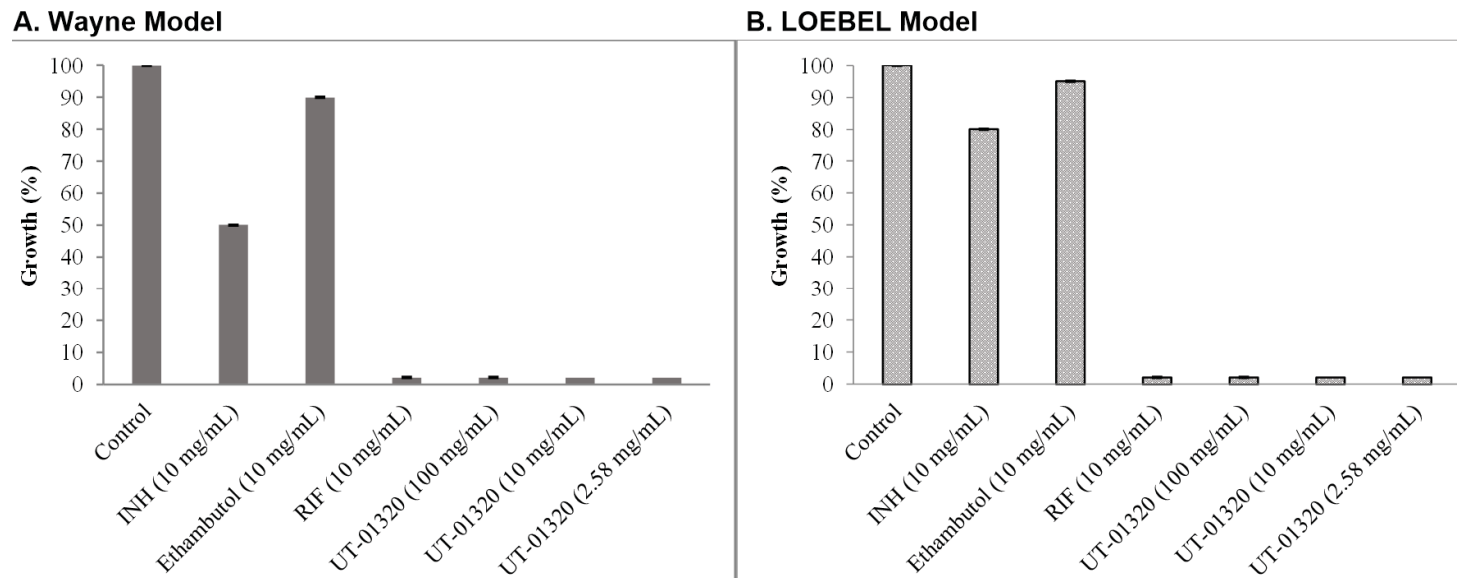

Figure 3-13. Bactericidal activity of UT-01320 against non-replicating Mtb evaluated in Wayne and nutrient deficient models.

UT-01320 (45) was tested at MIC, 4x MIC and 40x MIC in comparison with RIF, INH and ETB at 6x MIC. RIF - Rifampicin, INH - Isoniazid. 
$(\mathrm{MOI}=10)$ were treated with 45 (at $\mathrm{x} 2$ and $\left.\times 4 \mathrm{MIC}_{\text {LORA }}\right)$. After 24, 48, and $72 \mathrm{~h}$ of incubation, the relative intensity of the fluorescence was measured (emission wavelength $(581 \mathrm{~nm})$ ) via UV-vis spectroscopy (Figure 3-14A and B). Alternatively, the lysates were tenfold serially diluted in 7H10-S broth and inoculated on 7H11-S plates to determine the number of viable cell-associated $M t b$ to confirm the bactericidal effect of UT-01320 against intracellular $M t b$ in 72 h. 45 killed $M t b$ in infected macrophages at x2 $\mathrm{MIC}_{\mathrm{LORA}}$ concentrations within $72 \mathrm{~h}$. Figure 3-14A and B showed clearly that 45 kills intracellular $M t b$ as effectively as capreomycin (a 2 nd line TB drug). Bactericidal effect of 45 against the intracellular $M t b$ was distinguished from that of rifampicin in $72 \mathrm{~h}$. The intracellular activity of $\mathbf{4 5}$ was visualized via fluorescence confocal microscopy; significant reduction of intracellular $M t b$ was observed in 48-72 h compared to untreated control cultures (Figure 3-14C).

\section{Rapid Antimycobactericidal Activity of UT-01320}

Because of alteration of molecular target, we were interested in knowing whether a WecA inhibitor $\mathbf{4 5}$ remains rapidly bactericidal against replicating $M t b$ under aerobic conditions. The time-kill experiments were performed at two and fourfold the MIC of capuramycin, 45 and a few first-line TB drugs (RIF and INH)). Viable cell counting was performed at every $24 \mathrm{~h}$ for 14 days. CFUs were counted after 15 days of incubation at $37^{\circ} \mathrm{C}$. The rate of killing of $\mathbf{4 5}$ against $M t b$ was compared directly with the reference molecules and the time-kill assessments at 2x and 4x MIC concentrations are shown in Figure 3-15. The MurX inhibitor, capuramycin yielded $\log _{10} \mathrm{CFU} \mathrm{ml}{ }^{-1}$ reduction of 3.0 or greater for $M t b$ in 1 day and capuramycin killed $M t b$ completely in 2 days at $24.0 \mu \mathrm{g} \mathrm{ml}^{-1}$, albeit, RIF and INH required over 7 days to kill $50 \%$ of $M t b$ at $0.4 \mu \mathrm{g} \mathrm{ml}^{-1}$ and $1.0 \mu \mathrm{g} \mathrm{ml}^{-1}$, respectively (Figure 3-15A) $(91,188) .45 \mathrm{killed}>50 \%$ of $\mathrm{Mtb}$ within 3 days and $100 \%$ within 5 days at $3.0 \mu \mathrm{g} \mathrm{ml}^{-1}$ (2x MIC), and killed $100 \%$ of $M t b$ within 3 days at $6.0 \mu \mathrm{g} \mathrm{ml}^{-1}$ (4x MIC) (Figure 3-15B). Thus, the fast killing profile of 45 was similar to that of capuramycin; 45 killed Mtb faster than RIF and INH, but was less effective than capuramycin.

\section{Synergistic Effect of UT-01320 with MurX Inhibitors}

The synergistic or antagonistic activities of MurX inhibitors (capuramycin or SQ 641) and WecA inhibitor, 45 were assessed in vitro via micro dilution broth checkerboard technique $(189,190)$. In the checkerboard analyses of a combination of SQ 641 and $\mathbf{4 5}$, the FIC index range of 0.06 to 4 was observed for 47 combinations of two molecules out of 64 different concentrations. Table 3-5 summarizes the results of FIC index analyses for a combination of SQ 641 plus $\mathbf{4 5}$ and capuramycin plus $\mathbf{4 5}$ that showed synergistic combination $(\Sigma F I C<0.5)$. It was demonstrated that 45 exhibited synergy with SQ 641 and capuramycin that killed $M t b$ at much lower concentrations than the $\mathrm{MIC}_{\mathrm{A}}$ and $\mathrm{MIC}_{\mathrm{B}}$. The structure of $\mathbf{4 5}$ is closely related to the MurX inhibitor SQ 641. The PK/PD parameters of 45 may be correlated with those of SQ 641, and thus, a combination of two capuramycin 

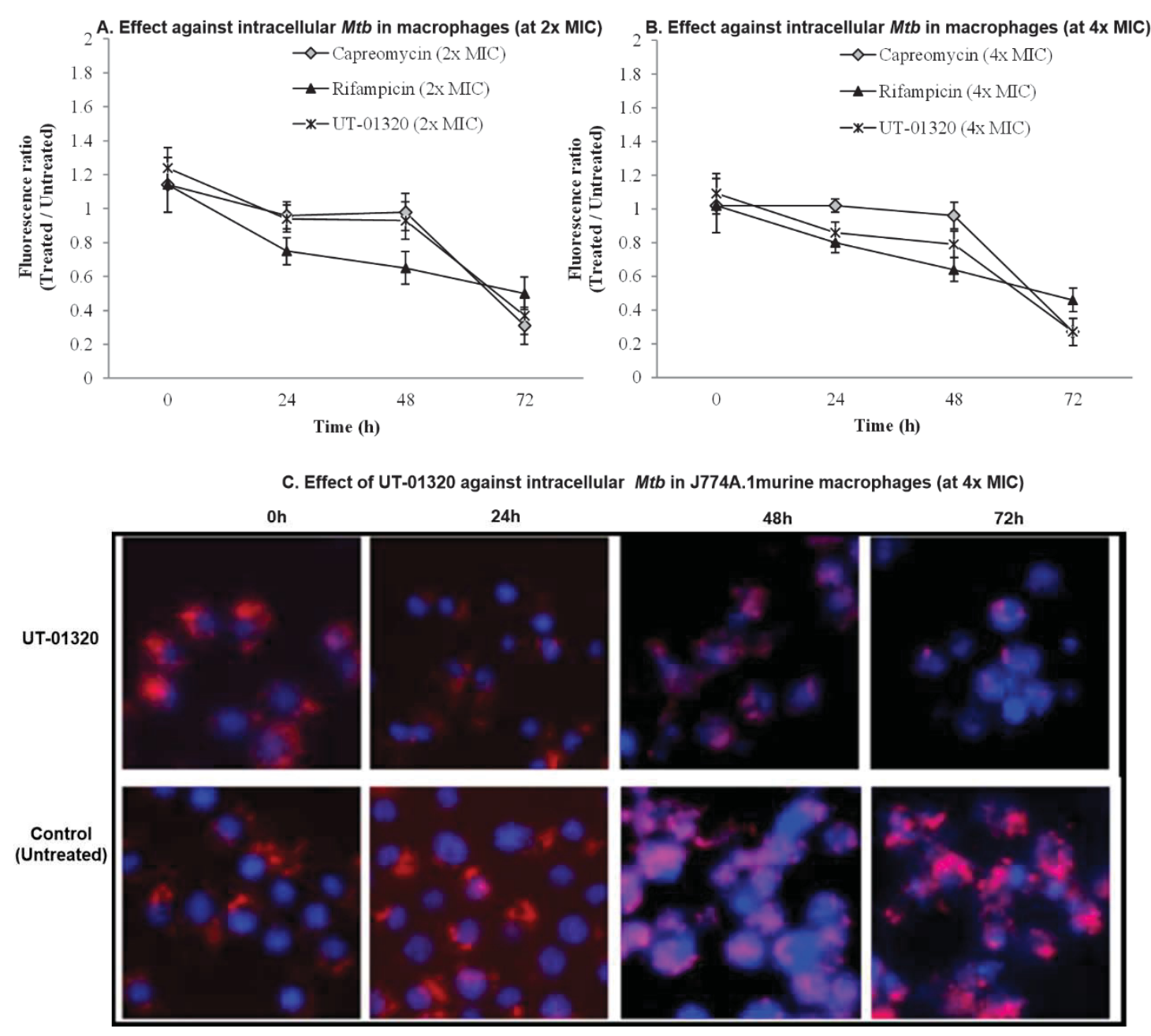

Figure 3-14. Effect of UT-01320 and representative TB drugs against intracellular Mtb CDC1551-tdTomato (a transformant Mtb CDC1551 containing tdTomato) in macrophages ( $\mathbf{J} 774 \mathrm{~A} .1$ cells).

A) Time-kill curve for intracellular $M t b$ at 2x MIC concentration. B) Time-kill curve for intracellular Mtb at 4x MIC concentration. C) Fluorescence confocal microscopy of J774A.1 murine macrophages infected with tdTomato (red) expressing Mtb after the treatment of $\mathbf{4 5}$ (at $4 \times \mathrm{XIC}$ ). Images were obtained for the treated and untreated cells for $72 \mathrm{~h}$. Fixed cells were stained with a blue fluorescent stain, DAPI (4',6-diamidino-2phenylindole) to visualize nuclei. 

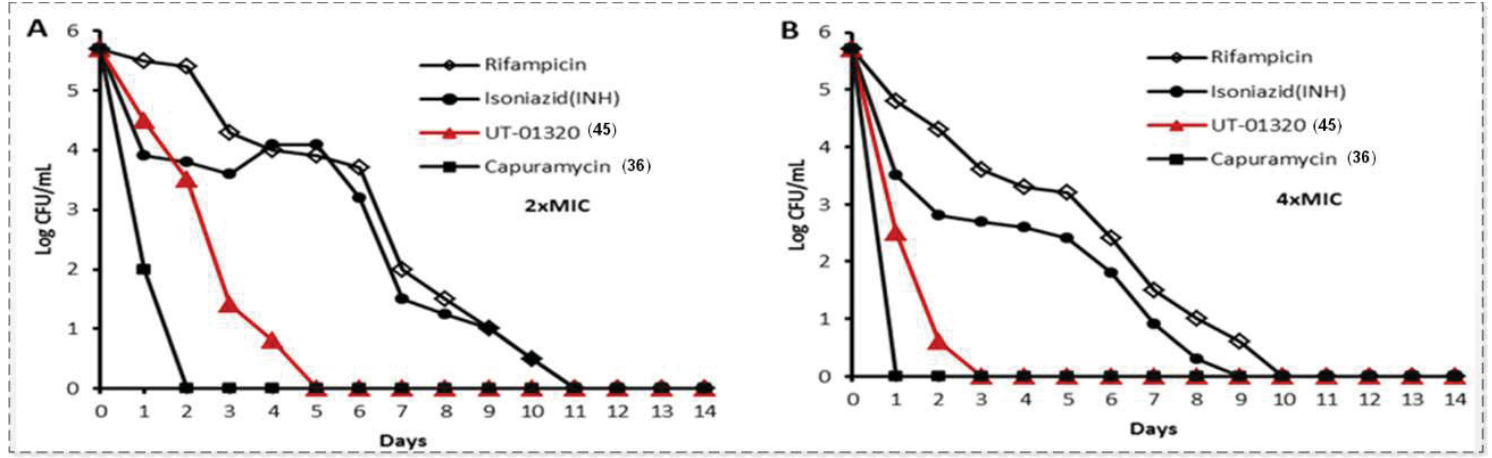

Figure 3-15. In vitro time-kill assessment of capuramycin, 45 and the first-line TB drugs (RIF and INH).

All the compounds were tested at 2x MIC and 4x MIC. RIF - Rifampicin, INH Isoniazid. 
Table 3-5. Fractional inhibitory concentration of a combination of two inhibitor molecules.

\begin{tabular}{lccc}
\hline Combination of A and B & $\begin{array}{c}\text { MIC }_{\mathbf{A}}(\mu \mathrm{g} / \mathbf{m L}) \\
\mathbf{M I C}_{\mathbf{B}}(\boldsymbol{\mu g} / \mathbf{m L})^{\mathbf{a}}\end{array}$ & $\begin{array}{c}\text { MIC }(\boldsymbol{\mu g} / \mathbf{m L}) \\
\mathbf{C}_{\mathbf{A}} \text { and } \mathbf{C B}_{\mathbf{B}} \mathbf{b}\end{array}$ & $\mathbf{\Sigma} \mathbf{F I C} \mathbf{C}^{\mathbf{c}, \mathbf{d}}$ \\
\hline A: SQ 641 (37) & 1.56 & 0.10 & 0.12 \\
B: UT-01320 (45) & 1.50 & 0.08 & \\
A: SQ 641 (37) & 1.56 & 0.10 & 0.17 \\
B: UT-01320 (45) & 1.50 & 0.16 & \\
A: SQ 641 (37) & 1.56 & 0.10 & 0.28 \\
B: UT-01320 (45) & 1.50 & 0.32 & \\
A: SQ 641 (37) & 1.56 & 0.10 & 0.49 \\
B: UT-01320 (45) & 1.50 & 0.645 & \\
A: SQ 641 (37) & 1.56 & 0.10 & 0.92 \\
B: UT-01320 (45) & 1.50 & 1.29 & \\
A: Capuramycin (36) & 12.50 & 1.563 & 0.18 \\
B: UT-01320 (45) & 1.50 & 0.08 & \\
A: Capuramycin (36) & 12.50 & 1.563 & 0.23 \\
B: UT-01320 (45) & 1.50 & 0.16 & \\
A: Capuramycin (36) & 12.50 & 0.78 & 0.28 \\
B: UT-01320 (45) & 1.50 & 0.32 & \\
A: Capuramycin (36) & 12.50 & 0.78 & 0.49 \\
B: UT-01320 (45) & 1.50 & 0.645 & \\
A: Capuramycin (36) & 12.50 & 0.78 & 0.92 \\
B: UT-01320 (45) & 1.50 & 1.29 & \\
\hline
\end{tabular}

NOTE: ${ }^{\mathrm{a}} \mathrm{MIC}_{\mathrm{A}}$ and $\mathrm{MIC}_{\mathrm{B}}$ are the MIC value of molecule $\mathrm{A}$ or $\mathrm{B}$ against $M t b,{ }^{\mathrm{b}} \mathrm{C}_{\mathrm{A}}$ and $\mathrm{C}_{\mathrm{B}}$ are concentrations of $\mathrm{A}$ and $\mathrm{B}$ in combination, ${ }^{\mathrm{c}} \Sigma \mathrm{FIC}$ index for the wells at growth-no growth interface, ${ }^{\mathrm{d}} \Sigma \mathrm{FIC}$ is the sum of fractional inhibitory concentration calculated by the equation $\Sigma \mathrm{FIC}=\mathrm{FIC}_{\mathrm{A}}+\mathrm{FIC}_{\mathrm{B}}=\mathrm{C}_{\mathrm{A}} / \mathrm{MIC}_{\mathrm{A}}+\mathrm{C}_{\mathrm{B}} / \mathrm{MIC}_{\mathrm{B}}$. 
analogs that have distinct drug targets is a great interest in evaluation of their synergistic interactions. These forward-looking biological characteristics for $\mathbf{4 5}$ promise that new TB drug leads will be discovered by extensive structure-activity relationship studies of 2'Omethylated capuramycin analogs.

\section{Spectrum of Activity of UT-01320}

As summarized in Table 3-6, 45 has a very narrow spectrum of activity; in growth inhibitory assays against Gram-positive and -negative bacteria including Mycobacterium spp., $\mathbf{4 5}$ killed $M t b$ strains selectively. It is interesting to note that unlike other capuramycin analogs, 45 did not kill a fast-growing M. smegmatis at $>200 \mu \mathrm{g} \mathrm{ml}^{-1}$ concentrations (for example, MICs $6.5 \mu \mathrm{g} \mathrm{ml}^{-1}$ and $25 \mu \mathrm{g} \mathrm{ml}^{-1}$ for capuramycin and UT01309 respectively).

\section{Conclusions}

We have discovered a novel capuramycin analog, UT-01320 (45); with WecA inhibitory activity that kills non-replicating $M t b$. This was achieved by screening a library of capuramycin analogs against MurX and WecA enzymes. Convenient high-throughput amenable screening methods for assay of WecA and MurX enzymes were developed. We have demonstrated MurX/MraY-catalyzed synthesis of neryl-lipid I-N ${ }^{\varepsilon}-\mathrm{C}_{6}$-fluorescent analogs (33 and 34) from Park's nucleotide- $\mathrm{N}^{\varepsilon}$-fluorescent analogs (29 and 32) with neryl phosphate. Biosynthesis of neryl-lipid I analogue was achieved, for the first time, in excellent yield with the MurX-containing membrane fraction (P-60). Similarly, neryllipid I-N ${ }^{\varepsilon}$-dansylthiourea could be biosynthesized via the different sources of MraY enzymes such as M. smegmatis, E. coli, and S. aureus. However, the purified MraY enzymes seem to be denaturing under the assay conditions developed for P-60 membrane fractions. A water-soluble lipid I generated in MurX assay media could be quantitated conveniently via reverse-phase HPLC without sophisticated extraction procedures. Signal-to-noise ratio of HPLC analyses of $\mathbf{2 9}$ and $\mathbf{3 3}$ is significantly high without using fluorescence detector. Furthermore, difference in the retention times between Park's nucleotide- $\mathrm{N}^{\varepsilon}$-dansylthiourea (29) and neryl-lipid $\mathrm{I}-\mathrm{N}^{\varepsilon}$-dansylthiourea (33) was more than $3.5 \mathrm{~min}$ and each assay analysis could be completed within $10 \mathrm{~min}$. We developed convenient methods for preparation of MurX/MraY enzymatic substrates, 29 and neryl phosphate (35), and thus, the substrates for the assays are available to screen a relatively large number of molecules in our laboratory. In order to determine usefulness of the MurX/MraY assay protocols developed here, we screened a 50-membered library including new uridyl peptides, and known positive- and negative-controls. MurX enzyme inhibitory activity of all positive-controls showed approximately equal to the $\mathrm{IC}_{50}$ values obtained with the previously reported methods. The negative-control molecules did not exhibit MurX inhibitory activity even at high concentrations. Because the assay method described here quantitates the MurX/MraY substrate and product simultaneously in each assay vial without extraction or separation, the errors in qualitative analyses of assay caused by quantitation of single-molecule (remaining molecule or converted molecule) 
Table 3-6. Spectrum of activity of UT-01320.

\begin{tabular}{lccc}
\hline \multirow{2}{*}{ Species and Strain } & \multicolumn{3}{c}{ MIC $(\boldsymbol{\mu g} / \mathbf{m L})^{\mathrm{a}}$} \\
\cline { 2 - 4 } & $\mathbf{U T - 0 1 3 2 0}(\mathbf{4 5})$ & $\mathbf{R I F}$ & $\mathbf{I N H}$ \\
\hline Mtb $\mathrm{H}_{37} \mathrm{Rv}$ & 2.50 & 0.01 & 0.57 \\
$M t b \mathrm{H}_{37} \mathrm{Rv} \mathrm{INHr}^{\mathrm{b}}$ & 2.45 & $0.10-0.20$ & $>8.0$ \\
$M t b \mathrm{H}_{37} \mathrm{Rv}$ RFPr & $>200$ & $>4.0$ & 0.05 \\
$M$. smegmatis ATCC607 & $>200$ & - & - \\
S. aureus ATCC6538D-5 & $>200$ & - & - \\
E. faecium ATCC 349 & $>200$ & - & - \\
E. coli ATCC 25019 & $>200$ & - & - \\
K. pneumonia ATCC 8047 & $>200$ & - & - \\
$P$. aeruginosa ATCC 27853 & & & \\
\hline
\end{tabular}

NOTE: ${ }^{\text {a }}$ The microplate alamar blue assay method was used, ${ }^{\mathrm{b}} \mathrm{INH}$-resistant $M t b$, ${ }^{\mathrm{c}} \mathrm{RFP}-$ resistant $M t b$, RIF - rifampicin, INH - isoniazid. 
and/or by complicated work-up procedures are diminished. In the screening of a small library of molecules using the described method, to date, a false positive or false negative result has not been identified.

We have been studying the molecular target for antimycobacterial nonMurX/MraY inhibitors identified in this program. High reliability for the MurX/MraY assay protocols described here will be a variable asset to identify selective MurX inhibitor molecules for development of new antibacterial agents.

We have developed convenient methods for preparation of WecA enzyme substrates and an assay amenable to high- and medium- throughput screens. We have validated the assay by screening a library of 50 capuramycin analogs and identifying $\mathbf{4 5}$ as a selective WecA inhibitor with bactericidal activity against replicating and nonreplicating $M t b$, as well as $M t b$ in macrophages. The efficacy of 45 against nonreplicating bacteria is especially significant, suggesting that the WecA enzyme is necessary to maintain viability of $M t b$ in a latent state $(102,191)$.

Methylation at the C2'-hydroxyl group of capuramycin ( $\mathrm{R}_{1}$ in Figure 3-12) alters the molecular target of capuramycin. 45 did not interfere with MurX, but exhibited bactericidal activity against nonreplicating (dormant) $M t b$ with the $\mathrm{MIC}_{\mathrm{LORA}} / \mathrm{MIC}_{\mathrm{MABA}}$ value of $1.72\left(\mathrm{MIC}_{\mathrm{LORA}} / \mathrm{MIC}_{\mathrm{MABA}}=7.35\right.$ for RIF). In preliminary assay screening of UT01320 against the potential drug targets for non-replicating $M t b$, it was observed that, UT-01320 did not inhibit electron transport systems, menaquinone biosynthesis, $\mathrm{Mtb}$ serine/threonine kinases (PKnA and B) and DNA gyrase. 


\section{CHAPTER 4. NOVEL PLEUROMUTILIN ANALOGS AS PROTEIN BIOSYNTHESIS INHIBITORS}

\section{Introduction}

Pleuromutilin (53), a strong binder of the 50S ribosomal subunit, was first isolated in 1952 from two Basidiomycete spp. Pleuromutilin analogs, tiamulin (54) and valnemulin (55) are FDA-approved drugs for use in veterinary medicine (192-194). Although azamulin (56) was developed for human use, due to its strong inhibition of cytochrome 450, it did not progress beyond phase I clinical trials. Retapamulin (57) (GSK) was approved by FDA in 2007 for topical (skin) use in humans $(117,195)$. An experimental drug, lefamulin (58) (Nabriva Therapeutics), entered Phase II clinical studies for systemic human use $(196,197)$. Strong antibacterial activity of pleuromutilins

against Gram-positive bacteria has been known since 1952; however, the effectiveness of pleuromutilin analogs against Mycobacterium spp. was not reported until 2003 (198).

Long et al. reported moderate activity of valnemulin against M. smegmatis (MIC 8-16 $\mu \mathrm{g} / \mathrm{mL}$ ) (133). To date, a large array of reported pleuromutilin derivatives are analogs whose structures are modified at the C14-ester side chain. Dong et al. reported $N$ benzylamine substituted pleuromutilin analogs with moderate anti-Mtb activity (60-100\% inhibition at $20 \mu \mathrm{M})(199)$. Lotesta et al. reported a tiamulin analog (59) which displayed moderate activity against $M t b(\mathrm{MIC} \sim 12.5 \mu \mathrm{g} / \mathrm{mL})(200)$. Ascher et al. filed a patent for several antimycobacterial pleuromutilin analogs whose structures are diversified the C2, C4, C6, C7, C12, or C14 positions (Figure 4-1) (201).

Pleuromutilins interfere with the peptidyl transferase center (PTC) of the 50s bacterial ribosomes. The co-crystal structure of Deinococcus radiodurans 50s ribosome with tiamulin (54) revealed that pleuromutilins bind two ribosomal key sites known as the 'A' site and ' $P$ ' site of the 50S subunit (118), resulting in the inhibition of peptide elongation, and thus, the cessation of bacterial growth. On the other hand, oxazolidinone antibacterial agents occupy solely the 'A' site of 50s ribosomes (112). Consequently, it is concluded that there is a lower probability of cross resistance between pleuromutilin and linezolid (198). Figure 4-2 illustrates the binding sites of pleuromutilin derivatives and linezolid on the bacterial 50S ribosomal subunit.

We have identified several pleuromutilin analogs that exhibit bactericidal acitivity against both replicating and non-replicating forms of Mtb. UT-800 (64) displayed the MICs of $<1.5 \mu \mathrm{g} / \mathrm{mL}$ in MABA and LORA assays. Unlike previously reported analogs, UT-800 is a narrow spectrum bactericidal molecule that showed focused activity against Mycobacterium spp. We have evaluated UT-800 in basic pharmacological in vitro experiments towards in vivo studies using the $M t b$ mouse infection models. 


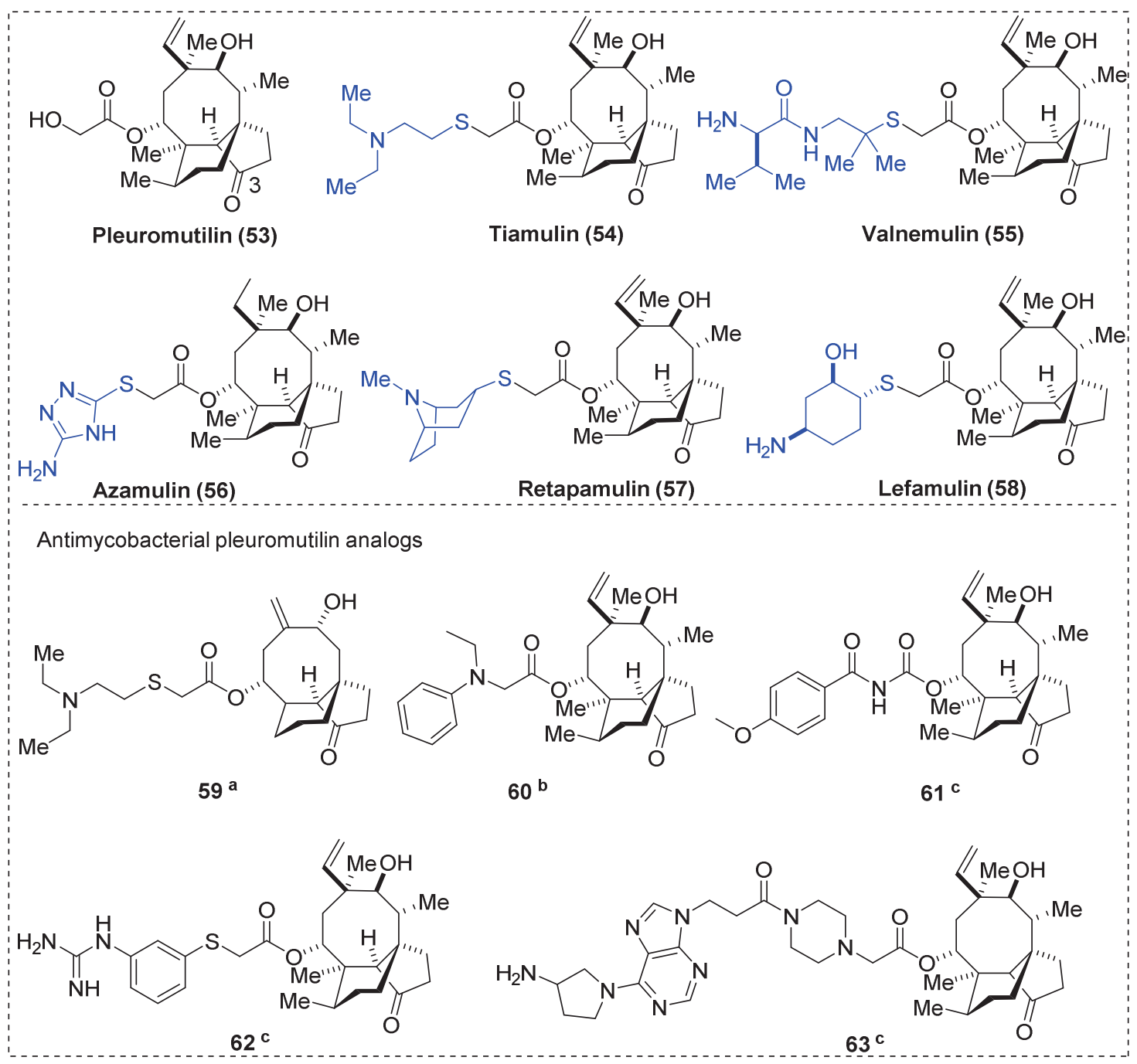

Figure 4-1. Representative structures of pleuromutilin analogs.

${ }^{\mathrm{a}}$ The anti-Mtb compound reported by Lotesta et al, ${ }^{\mathrm{b}}$ Dong et al, ${ }^{\mathrm{c}}$ Ascher et al. 


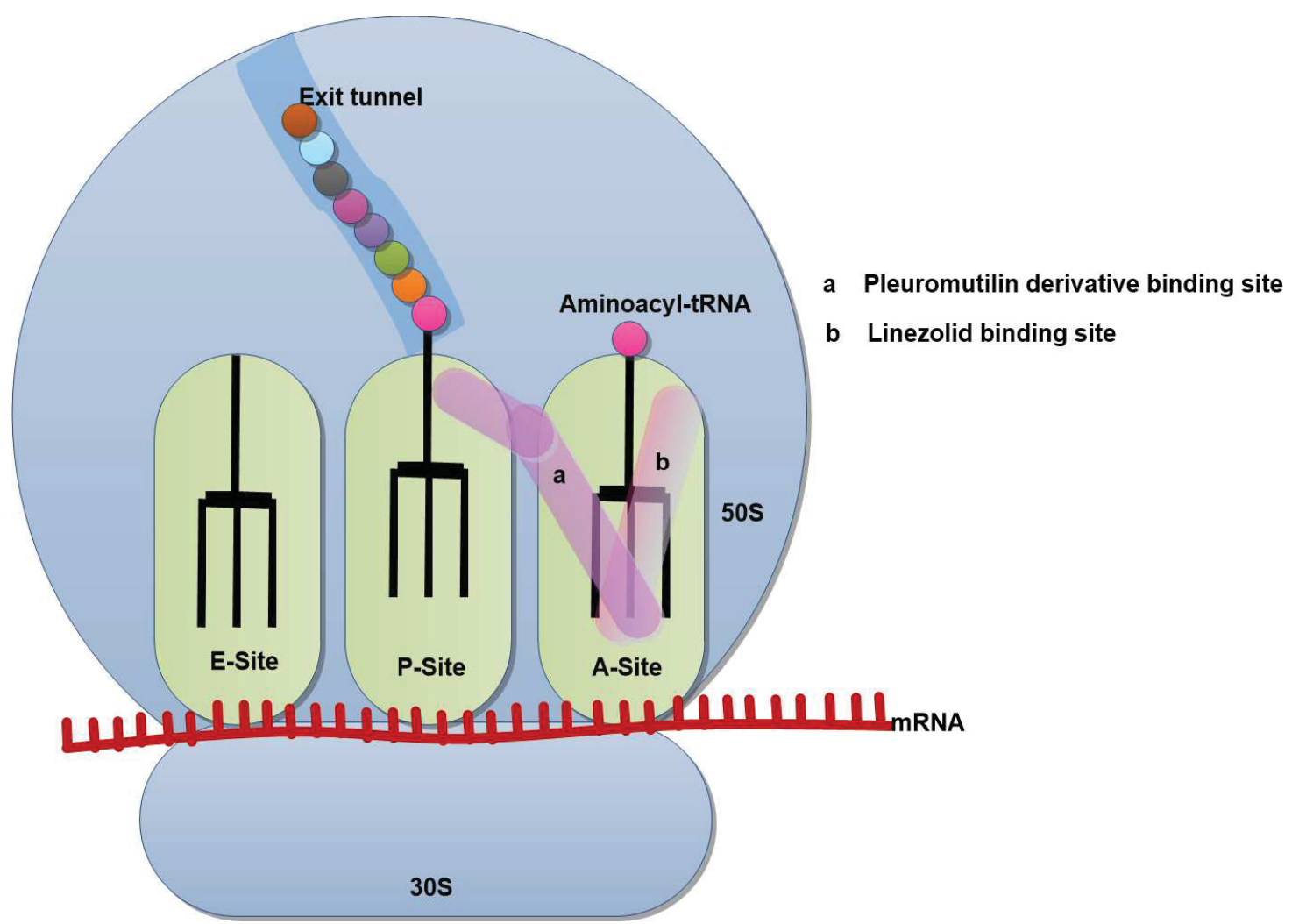

Figure 4-2. Binding sites of pleuromutilin derivatives and linezolid on 50s ribosome.

Pleuromutilin derivatives interfere with both the A- and P- sites of bacterial 50s ribosome; a - Pleuromutilin binding site, $\mathbf{b}$ - Linezolid binding site. 


\section{Results and Discussion}

\section{Screening of Pleuromutilin Analogs against $M t b$}

To identify new pleuromutilin analogs with activity towards $M t b$, we have generated over 50 analogs whose structures contained the methoxyamine-oxime (Figure 4-3A), hydroxylamine-oxime (Figure 4-3B), or 3-hydroxy (Figure 4-3C) core structure. These structures were further diversified by the reduction of the double bond (C16 position), amide formations or reductive aminations. The generated molecules were evaluated in the growth inhibitory assays against $M t b\left(\mathrm{H}_{37} \mathrm{Rv}\right)$. We identified eight pleuromutilin analogs which exhibited the MIC value $<12.5 \mu \mathrm{g} / \mathrm{mL}$ against $\mathrm{Mtb}$ (Figure 4-3D). Out of the eight molecules UT-800 (64), UT-810 (65) and UT-815 (66) exhibited the lowest growth inhibitory activities against Mtb (MIC 0.78-1.56 (MABA) and 1.041.98 (LORA).

\section{Synthesis of UT-800, UT-810 and UT-815}

In order to confirm anti-Mtb activity of three potent compounds from the screenings, these molecules were resynthesized in our group. Their syntheses are illustrated in Scheme 1 (Figure 4-4). The C3-carbonyl group of pleuromutilin (53) was reduced with $\mathrm{NaBH}_{4}$ to form the secondary alcohol with the $R$-configuration exclusively. The primary alcohol of the C14-side chain was tosylated with $\mathrm{TsCl}$ and DMAP to afford 72 in Figure 4-4 in 90\% overall yield. Thioether formation of 72 with 1-amino-2methylpropane-2-thiol was accomplished according to a reported condition, yielding the free amine 73 in Figure 4-4 in 90\% yield (202,203). Amide-forming reaction of $\mathbf{7 3}$ with Boc-D-Leu-OH under glyceroacetonide-Oxyma, EDCI, and $\mathrm{NaHCO}_{3}$ in $\mathrm{DMF}-\mathrm{H}_{2} \mathrm{O}$ followed by reduction with $\mathrm{H}_{2} / \mathrm{Pd}-\mathrm{C}$ in $\mathrm{MeOH}-\mathrm{EtOAc}$. Deprotection of the Boc group with $4 N \mathrm{HCl}$ afforded UT-800 (64) (204-206). Compounds 65 and 66 were synthesized by tosylation of the primary alcohol of the $\mathrm{C} 14$-side chain with $\mathrm{TsCl}$ and DMAP to afford 74 in Figure 4-4, and the corresponding thioether formation of 74 with 1-amino-2methylpropane-2-thiol was accomplished, yielding the free amine 75. Corresponding amide formation with Boc-D-Leu-OH and subsequent Boc-deprotection yielded UT-810 (65). The Boc protected derivative was reduced with $\mathrm{H}_{2} / \mathrm{Pd}-\mathrm{C}$ in $\mathrm{MeOH}-\mathrm{EtOAc}$ followed by Boc-deprotection yielded UT-815 (66). The analogs synthesized in Scheme 1 were purified via reverse-phase HPLC.

\section{Effectiveness of UT-800, 810 and 815 against Replicating and Non-replicating $M t b$ via MABA and LORA Assays}

The MIC values of the analogs were determined against $M t b \mathrm{H}_{37} \mathrm{Rv}$ in both aerobic and anaerobic conditions via MABA and LORA assays, respectively. The analogs 64, 65 and 66 exhibited MIC $\mathrm{MABA}_{\text {of }} 0.78-1.46 \mu \mathrm{g} / \mathrm{mL}$ and $\mathrm{MIC}_{\text {LORA }}$ of 1.04-1.98 $\mu \mathrm{g} / \mathrm{mL}$ (Table 4-1). The ratio of $\mathrm{MIC}_{\mathrm{LORA}} / \mathrm{MIC}_{\mathrm{MABA}}$ for the three compounds was 


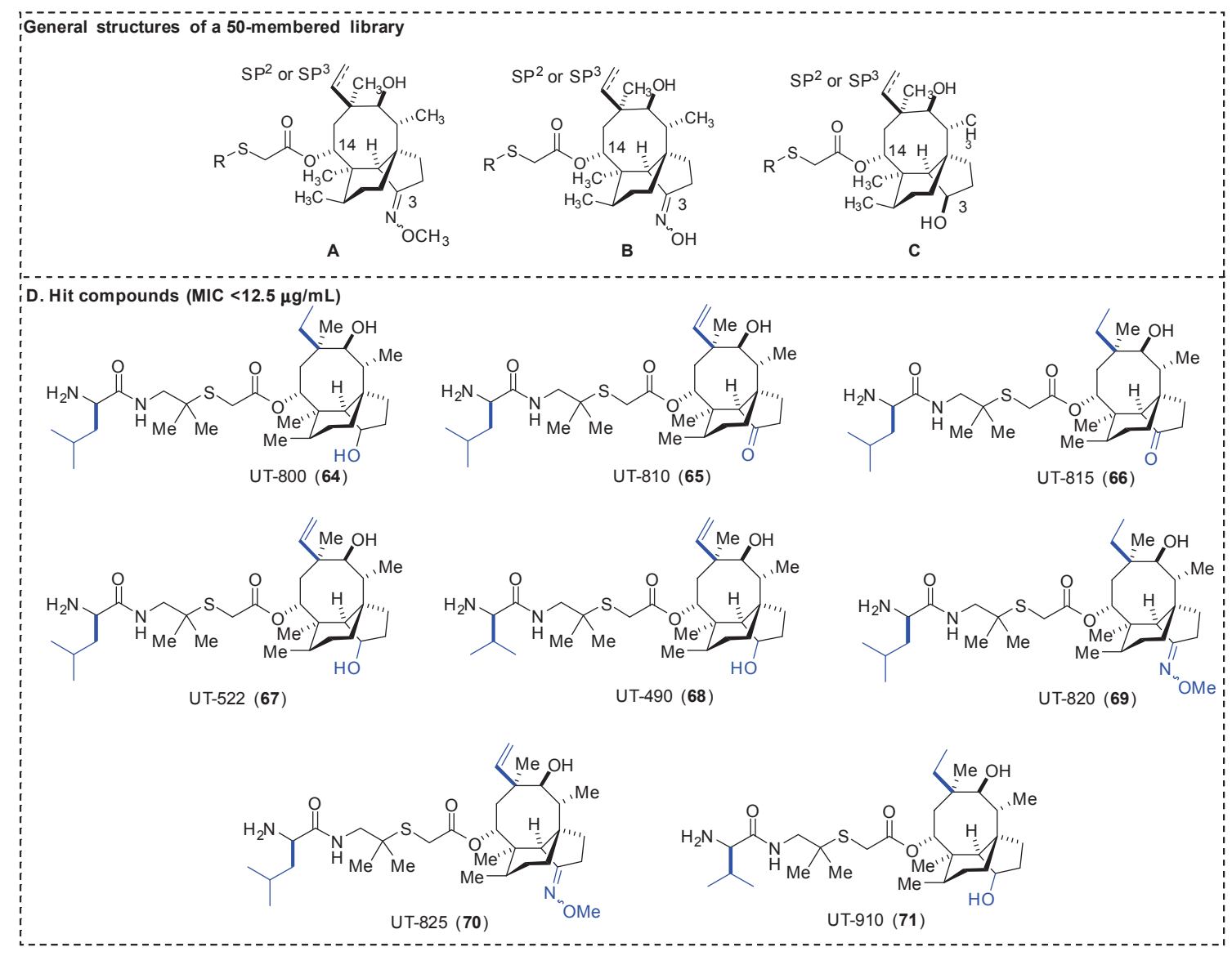

Figure 4-3. General structures of pleuromutilin derivatives and Anti-Mtb molecules.

The structures were diversified by the reduction of the double bond (C16 position), amide formations and reductive aminations at $\mathrm{C} 14$-side chain and reduction at $\mathrm{C} 3$ position. 


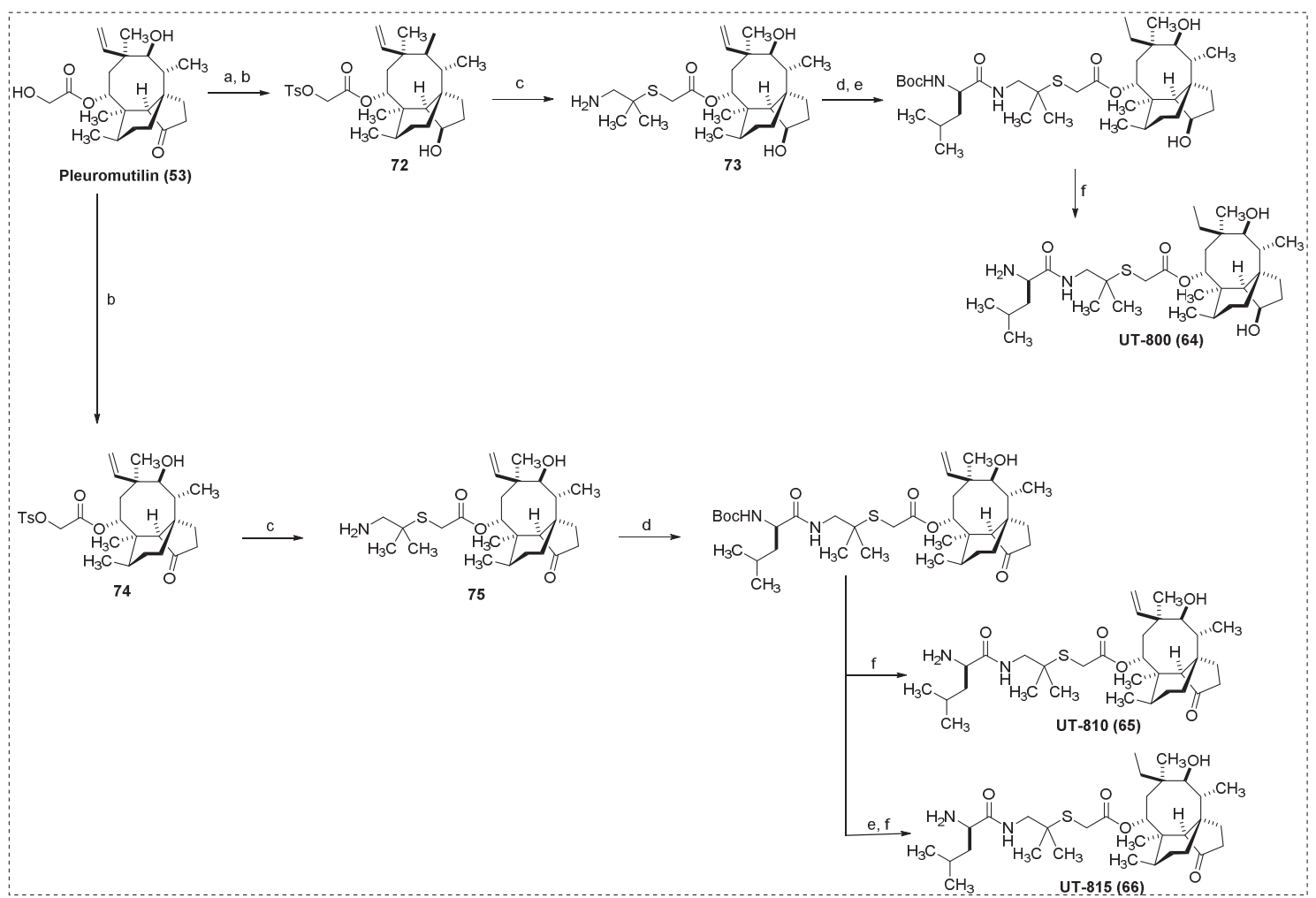

Figure 4-4. Scheme 1: syntheses of pleuromutilin analogs UT-800, UT-810 and UT-815.

Conditions - a) $\mathrm{NaBH}_{4}, \mathrm{MeOH}, 0{ }^{\circ} \mathrm{C}$. b) TsCl, DMAP, $\mathrm{CH}_{2} \mathrm{Cl}_{2}, 0{ }^{\circ} \mathrm{C}$. c) 1-amino-2methylpropane-2-thiol, $1 \mathrm{M} \mathrm{NaOH}, \mathrm{nBu}_{4} \mathrm{NBr}, \mathrm{THF} / \mathrm{H}_{2} \mathrm{O}(1: 1), 50{ }^{\circ} \mathrm{C}$. d) Boc-D-Leu-OH, Glyceroacetonide-Oxyma, EDCI, $\mathrm{NaHCO}_{3}$, DMF-H $\mathrm{H}_{2} \mathrm{O}$ (9/1). e) $\mathrm{H}_{2}, \mathrm{Pd} / \mathrm{C}, \mathrm{MeOH}-\mathrm{EtOAc}$. f) $\mathrm{HCl}$, Dioxane. 
Table 4-1. Anti-Mtb activity and cytotoxicity of pleuromutilin analogs.

\begin{tabular}{|c|c|c|c|c|}
\hline \multirow{2}{*}{ Entry } & \multirow{2}{*}{ Compounds } & \multicolumn{2}{|c|}{ MIC $(\mu \mathrm{g} / \mathrm{mL})$ against $M t b$} & \multirow{2}{*}{$\begin{array}{c}\mathrm{IC}_{50}(\mu \mathrm{g} / \mathrm{mL}) \\
\text { against Vero Cells }\end{array}$} \\
\hline & & MABA $^{\mathrm{a}}$ & LORA $^{b}$ & \\
\hline 1 & UT-800 (64) & 0.83 & 1.20 & 10.45 \\
\hline 2 & UT-810 (65) & 1.56 & 1.98 & 13.96 \\
\hline 3 & UT-815 (66) & 0.78 & 1.04 & 5.26 \\
\hline 4 & UT-522 (67) & 3.06 & 3.46 & 25 \\
\hline 5 & UT-490 (68) & 6.25 & - & 45.3 \\
\hline 6 & UT-820 (69) & 5.81 & 2.76 & 5 \\
\hline 7 & UT-825 (70) & 8.18 & - & 3.13 \\
\hline 8 & UT-910 (71) & 12.5 & - & 5.26 \\
\hline 9 & Valnemulin (55) & 3.13 & - & 15.59 \\
\hline 10 & RIF & $0.01-0.2$ & 0.05 & 102.3 \\
\hline 11 & $\mathrm{INH}$ & 0.57 & 50.24 & $>200$ \\
\hline 12 & Tunicamycin (38) & 12.5 & - & 0.18 \\
\hline
\end{tabular}

NOTE: ${ }^{\mathrm{a}}$ The microplate alamar blue assay method was used, ${ }^{\mathrm{b}}$ Low oxygen recovery assay. 
between $1.27-1.45$, which is closer to the ideal value of 1 or $<1$. The identified molecules are analogs of valnemulin and they exhibited a superior inhibitory profile against $M t b \mathrm{H}_{37} \mathrm{Rv}$ compared to valnemulin.

In order to obtain insights into potential toxicity of identified inhibitor molecules, all antimycobacterial pleuromutilin analogs were evaluated in in vitro cytotoxicity assays against Vero monkey kidney cells. The toxicity profile of 64, 65 and 66 was similar to that of valnemulin (55). We are currently evaluating the acute toxicity ( $\mathrm{LD}_{50}$ value) of 64 via ICR female mice (4 weeks old).

\section{Effectiveness of UT- 800, 810 and 815 against Intracellular Mtb}

The activity of the three analogs against intracellular $M t b$ was evaluated. Murine macrophage cell J774A.1 infected by a transformant Mtb CDC1551 containing tdTomato $(\mathrm{MOI}=10)$ were treated with UT-800, -810 and -815 (at x2 and x4 MIC LORA). After 24, 48 , and $72 \mathrm{~h}$ of incubation, the relative intensity of the fluorescence was measured (emission wavelength $(581 \mathrm{~nm})$ ) via $\mathrm{UV}$-vis spectroscopy (Figure 4-5A and B). Alternatively, the lysates were ten-fold serially diluted in 7H10-S broth and inoculated on 7H11-S plates to determine the number of viable cell-associated $M t b$ to confirm the bactericidal effect of the analogs against intracellular $M t b$ in $72 \mathrm{~h}$. The analogs killed $M t b$ in infected macrophages at $x 2 \mathrm{MIC}_{\text {LORA }}$ concentrations within $72 \mathrm{~h}$. Figure 4-5A and $\mathbf{B}$ shows that 64, 65 and 66 kill intracellular $M t b$ better than rifampicin. INH did not kill intracellular Mtb at x2 and x4 MIC.

\section{Rapid Antimycobactericidal Activity of UT-800, 810 and 815}

The time-kill experiments were performed at two and four-fold the MIC of $\mathbf{6 4 , 6 5}$, 66 and two first-line TB drugs (RIF and INH). Viable cell counting was performed at every $24 \mathrm{~h}$ for 16 days. CFUs were counted after 15 days of incubation at $37^{\circ} \mathrm{C}$. The rate of killing of analogs against $M t b$ was compared with the reference molecules, and the time-kill assessments at x2 MIC concentrations are shown in Figure 4-6. The killing profile of the new analogs was similar to that of RIF and INH. RIF and INH required 7 days to kill $50 \%$ of Mtb at $0.4 \mu \mathrm{g} / \mathrm{ml}$ (x2 MIC) and $1.0 \mu \mathrm{g} / \mathrm{ml}$ (x2 MIC), respectively.

\section{Spectrum of Activity of UT-800, 810 and 815}

As summarized in Table 4-2, the spectrum of activity of the three pleuromutilin analogs $(64,65$ and 66) was determined by assaying against 15 bacterial strains including Gram-positive, -negative bacteria and Mycobacterium spp. We concluded that 64, 65 and $\mathbf{6 6}$ kills Mycobacterium species selectively and are especially effective in killing Mtb at low concentrations. 

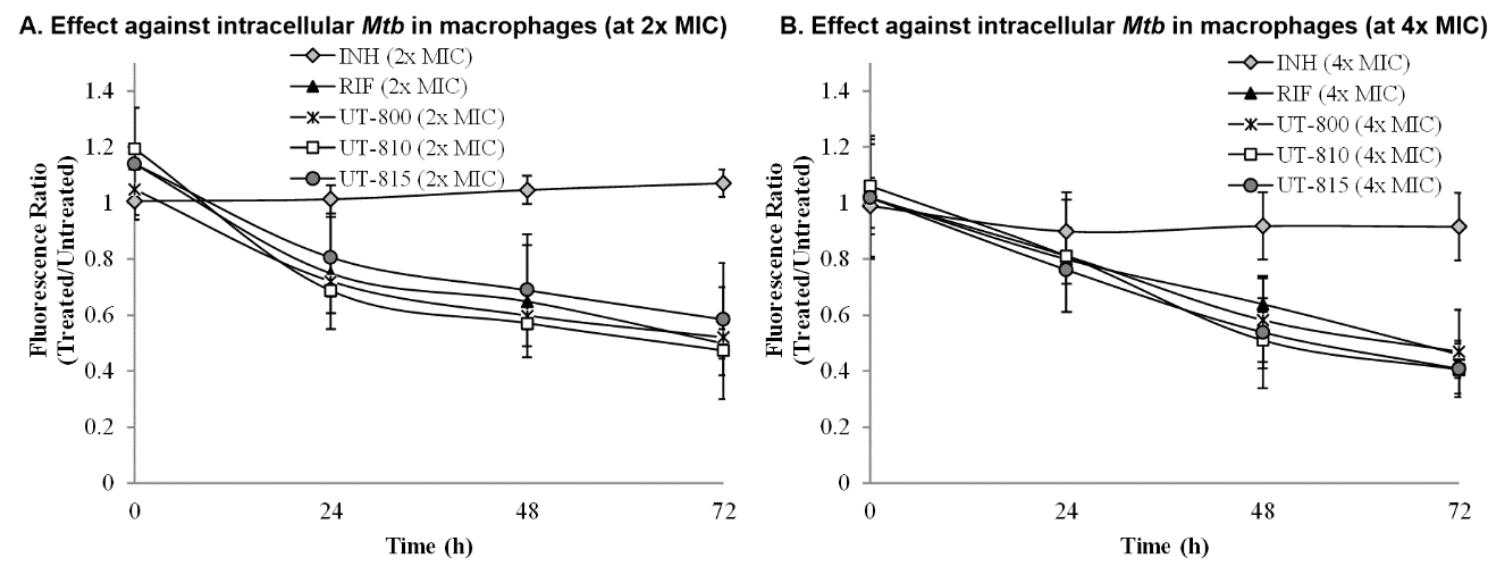

Figure 4-5. Effect of pleuromutilin analogs and representative TB drugs against intracellular Mtb CDC1551-tdTomato (a transformant Mtb CDC1551 containing tdTomato) in macrophages ( $\mathbf{J 7 7 4 A . 1}$ cells).

A) Time-kill curve for intracellular $M t b$ at 2x MIC concentration. B) Time-kill curve for intracellular Mtb at 4x MIC concentration.

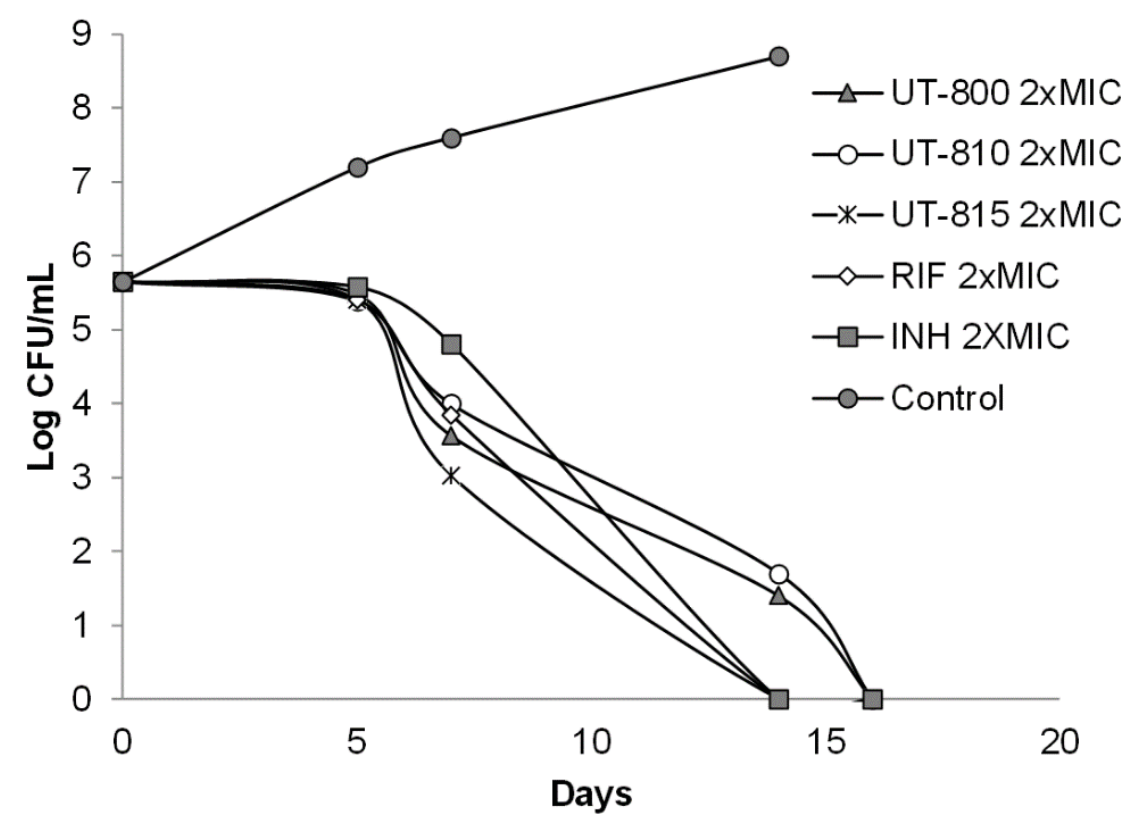

Figure 4-6. In vitro time-kill assessment of valnemulin (55), 64, 65, 66 and the first-line TB drugs (RIF and INH). 
Table 4-2. Spectrum of activity of UT-800, UT-810 and UT-815.

\begin{tabular}{|c|c|c|c|c|c|c|c|c|}
\hline \multirow{2}{*}{ Species and Strain } & \multicolumn{8}{|c|}{$\operatorname{MICs}(\mu \mathrm{g} / \mathrm{mL})^{\mathrm{a}}$} \\
\hline & UT-800 (64) & UT-810 (65) & UT-815 (66) & VAL (55) & RIF & INH & CPR & LIN \\
\hline$M t b \mathrm{H}_{37} \mathrm{Rv}$ & 0.83 & 1.46 & 0.77 & 3.13 & 0.2 & 0.57 & 3.13 & 1.61 \\
\hline M. smegmatis ATCC 607 & 25 & $>25$ & 25 & 25 & $>25$ & - & 25 & 0.8 \\
\hline S. aureus ATCC 25923 & $>25$ & $>25$ & $>25$ & $>25$ & $>25$ & - & $>25$ & $>25$ \\
\hline S. aureus BAA 2094 & $>25$ & $>25$ & $>25$ & $>25$ & 0.2 & - & $>25$ & $>25$ \\
\hline S. aureus BAA 1683 & $>25$ & $>25$ & $>25$ & $>25$ & 0.2 & - & $>25$ & 25 \\
\hline S. aureus BAA 44 & $>25$ & $>25$ & $>25$ & $>25$ & 25 & - & $>25$ & $>25$ \\
\hline E. faecium ATCC 349 & $>25$ & $>25$ & $>25$ & $>25$ & $>25$ & - & $>25$ & $>25$ \\
\hline E. faecalis ATCC 19433 & 12.5 & 25 & 25 & 12.5 & 1.56 & - & $>25$ & 25 \\
\hline S. pneumoniae ATCC 6301 & $>25$ & $>25$ & $>25$ & $>25$ & $>25$ & - & $>25$ & $>25$ \\
\hline S. salivarius ATCC 7073 & $>25$ & $>25$ & $>25$ & $>25$ & $>25$ & - & $>25$ & $>25$ \\
\hline B. subtilis ATCC 6051 & $>25$ & 25 & 25 & 25 & 0.4 & - & $>25$ & 6.25 \\
\hline E. coli ATCC 10798 & $>25$ & $>25$ & $>25$ & $>25$ & 25 & - & $>25$ & $>25$ \\
\hline K. pneumonia ATCC 8047 & 25 & $>25$ & $>25$ & 25 & 3.13 & - & $>25$ & $>25$ \\
\hline P. aeruginosa ATCC 27853 & $>25$ & $>25$ & $>25$ & $>25$ & - & - & - & - \\
\hline A.baumannii ATCC 19606 & 3.13 & 25 & 12.5 & 6.25 & 3.13 & - & $>25$ & $>25$ \\
\hline
\end{tabular}

NOTE: ${ }^{a}$ The microplate alamar blue assay method was done, VAL - Valnemulin, RIF - Rifampicin, INH - Isoniazid, CPR Capreomycin, LIN - Linezolid. 


\section{In vitro Metabolic Stability of UT-800, UT-810, and UT-815}

In our study of in vitro metabolism of the three potent pleuromutilin analogs in rat liver microsomes, we observed a striking difference in half-life $\left(\mathrm{t}^{1} / 2\right)$ between $\mathrm{C} 3$ hydroxyl compound 64 and C3-carbonyl compounds 65 and 66. The half-life of 64 was determined to be $>60 \mathrm{~min}$, on the other hand, $t_{1 / 2}$ of Ut-810 and -815 were $1.29 \mathrm{~min}$. As such, in vitro half-life was significantly extended by reduction of the C3-carbonyl group of valnemulin (Figure 4-7). We have also observed the same trend of increased in vitro half-life by reducing the C3-carbonyl group of valnemulin to hydroxyl group in our antiAcinetobacter baumannii analog, UT-490 (68) (207).

\section{Prediction of Intestinal Permeability of UT-800 via Caco-2 Permeability Assay}

The suitability of $\mathbf{6 4}$ for oral dosing was evaluated by using a bidirectional Caco-2 permeability assay by Cyprotex to predict in vivo intestinal permeability and to investigate drug efflux. This established in vitro model predicts the in vivo absorption of drugs across the gut wall by measuring the rate of transport across the Caco- 2 cell line that resembles the intestinal epithelial cells (208). 64 was added to the apical side of the membrane and the transport of the compound across the monolayer was monitored over a $2 \mathrm{~h}$ time period. The transport of $\mathbf{6 4}$ in the apical to basolateral direction (A-B) as well as the basolateral to apical direction (B-A) was assessed to determine an efflux ratio which provides an indicator as to whether $\mathbf{6 4}$ undergoes active efflux. The permeability coefficient $\left(\mathrm{P}_{\mathrm{app}}\right)$ was determined to be $3.39 \times 10^{-6} \mathrm{~cm} / \mathrm{s}$ from A-B and $18.3 \times 10^{-6} \mathrm{~cm} / \mathrm{s}$ from B-A (Figure 4-8). Efflux ratio $\left(\mathrm{P}_{\mathrm{app}}(\mathrm{B}-\mathrm{A}) / \mathrm{P}_{\mathrm{app}}(\mathrm{A}-\mathrm{B})\right)$ was determined to be 5.44 . Ranitidine was used as a low permeability control $\left(\mathrm{P}_{\text {app }}<1\right)$ and warfarin was used as a high permeability control $\left(\mathrm{P}_{\mathrm{app}}>1\right)$. Talinolol, a known $\mathrm{P}$-gp substrate, was screened as a control compound to confirm that the cells are expressing functional efflux proteins $\left(\mathrm{P}_{\text {app }}(\mathrm{B}-\mathrm{A}) / \mathrm{P}_{\text {app }}(\mathrm{A}-\mathrm{B})=76.8\right)$. $\mathrm{A} \mathrm{P}_{\text {app }}$ rate coefficient $>1$ and efflux ratio apprx 5 indicates that 64 has permeability across the membrane with moderate levels of efflux (an efflux ratio greater than two indicates that drug efflux is occurring).

\section{Genetic Analysis of UT-490 to Study the Resistance Mechanism}

It is well established that the pleuromutilin derivatives target the peptidyl transferase center of the 50S ribosomal protein L3 (rplC), inhibiting protein biosynthesis (118,119,198,209-213). Previously, other groups reported multiple mutations in $\mathrm{rplC}$ of $S$. aureus that can define a region of $r p l C$ capable of causing decreased susceptibility of the pleuromutilin derivative in $S$. aureus $(118,210,211)$. We have evaluated an antiAcinetobacter baumannii analog, UT-490 (68) for its potential mechanism of resistance. UT- 490 has also displayed moderate activity against Mtb (Entry 5 in (Table 4-1) (207). In order to identify a potential mechanism of resistance to UT-490 (68), we isolated the chromosomal DNA from the resistant mutant (UT-490 R $16 x \mathrm{xIC}$ ) and its parental wildtype control $A$. baumannii (ATCC19606). The $r p l C$ gene fragment was amplified using 


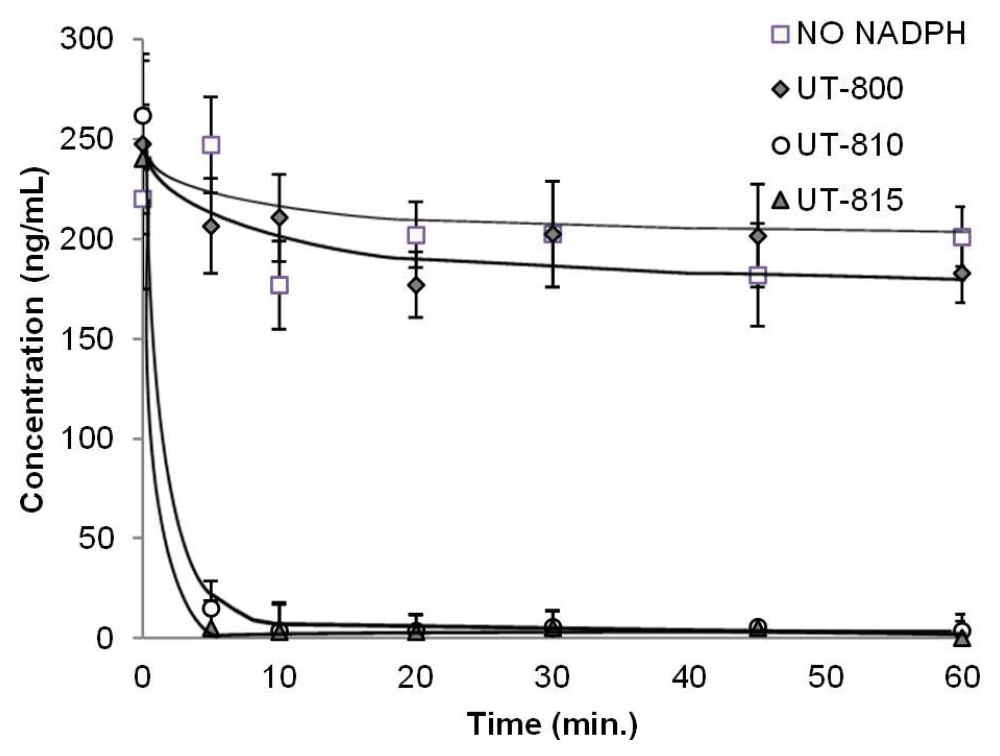

Figure 4-7. In vitro metabolic stability of pleuromutilin analogs $(64,65$, and 66). Verapamil was utilized as a control compound, and the half-life $\left(\mathrm{t}_{1 / 2}\right)$ of verapamil was determined to be $10 \mathrm{~min}$. 
A

\begin{tabular}{|c|c|c|c|c|c|}
\hline Compound & $\begin{array}{c}\text { Test } \\
\text { Concentration } \\
(\boldsymbol{\mu} \mathbf{M})\end{array}$ & $\begin{array}{c}\text { Assay Duration } \\
\mathbf{( h )}\end{array}$ & $\begin{array}{c}\text { Mean } \mathbf{A} \rightarrow \mathbf{B} \\
\mathbf{P}_{\text {app }}(\mathbf{1 0} \\
\mathbf{c m} / \mathbf{6})\end{array}$ & $\begin{array}{c}\text { Mean } \mathbf{B} \rightarrow \mathbf{A} \\
\mathbf{P}_{\text {app }}(\mathbf{1 0}-\mathbf{6} \\
\mathbf{c m} / \mathbf{s})\end{array}$ & Efflux Ratio \\
\hline Ranitidine & 10.0 & 2.00 & 0.237 & 1.14 & 5.00 \\
\hline Talinolol & 10.0 & 2.00 & 0.0517 & 3.92 & 76.8 \\
\hline UT-800 & 10.0 & 2.00 & 3.39 & 18.3 & 5.44 \\
\hline Warfarin & 10.0 & 2.00 & 35.2 & 28.0 & 0.794 \\
\hline
\end{tabular}

B

$\square \mathrm{A} \rightarrow \mathrm{B} \quad \mathrm{BB} \rightarrow \mathrm{A}$

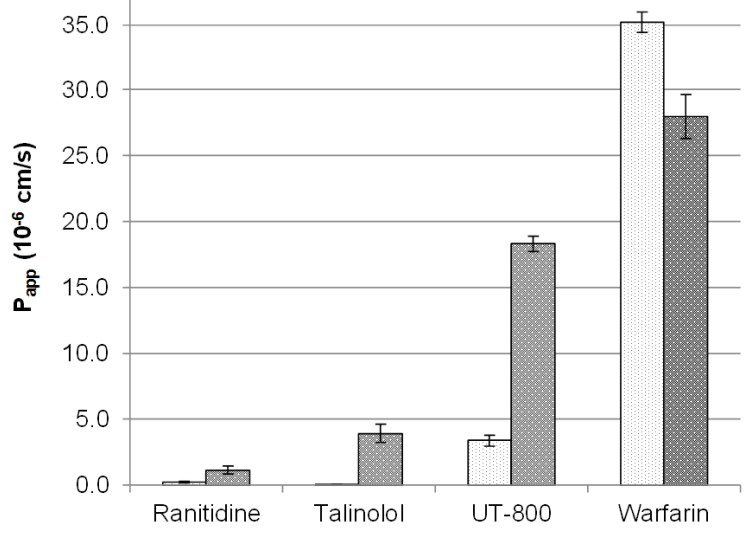

Figure 4-8. In vitro Caco-2 permeability assay to predict intestinal permeability of UT-800 (64).

A) Experimental data for Caco-2 assay. B) 64 and control compounds were plotted against the permeability rate coefficient $\left(\mathrm{P}_{\text {app }}\left(10^{-6} \mathrm{~cm} / \mathrm{s}\right)\right.$. 
A. baumannii rplC specific primers and sequenced (214,215). The DNA sequencing results were blasted against $r p l C$ DNA sequence of $A$. baumannii in the NIH genetic sequence database. The DNA sequence alignment revealed a C456A single nucleotide mutation, which corresponded to $\mathrm{N} 152 \mathrm{~K}$ mutation in the protein sequence of RplC

(Figure 4-9). UT- 800 (64) is structurally similar to 68; the D-valine moiety at the C14 side chain is replaced by D-leucine and the C16 vinyl moiety is saturated (Figure 4-3). The mode of action of $\mathbf{6 4}$ is believed to be the same as that of $\mathbf{6 8}$; the transcription/translation coupled luciferase reporter assays showed that the both moleuces inhibited protein biosynthesis by $55-67 \%$ at $0.1 \mu \mathrm{g} / \mathrm{mL}$ concentration.

\section{Conclusions}

We identified a new pleuromutilin derivative UT-800 (64) that displays a strong antimycobacterial activity against $M t b$. UT-800 kills $M t b$ in both replicating and nonreplicating forms with 0.83 and $1.20 \mu \mathrm{g} / \mathrm{mL}$ concentration, respectively, and it effectively kills the intracellular $M t b$; the time-killing profile of $\mathbf{6 4}$ is similar to that of RIF. Interestingly, spectrum of activity of UT-800 is focused against Mycobacterium spp. that is an attractive characteristic to progress anti-TB drugs. UT-800 is a C3-reduced analog of pleuromutilin that significantly extends the in vitro half-life $\left(t_{1 / 2}>60 \mathrm{~min}\right)$ with rat liver microsomes. The gene analyses of $A$. baumannii strain that is resistant to $\mathbf{6 8}$ revealed a single mutation in the $r p l C$ gene, suggesting that an $M t b$ mutant against 64 may exhibit a similar resistant mechanism.

The pleuromutilin analog, 64 exhibited permeability across Caco-2 epithelial monolayers ( $\mathrm{P}_{\text {app }}$ rate coefficient $>1$ ) with moderate levels of efflux (an efflux ratio of approximately 5). These Caco-2 permeability data predict an oral bioavailability for $\mathbf{6 4}$ in vivo. Pharmacokinetics, oral bioavailability and in vivo toxicity of 64 are currently being studied in our group using preclinical animal models.

\begin{tabular}{lll} 
Ouery & 367 & FOGGVKRWNERTODATHGNSVSHRVGGSTOKOTPGRVEKGKKMAGHLGDERVTVOGLEI \\
\hline Sbjct & 121 & FOGGGGRVINRRTODATHGNSVSHRVLGSTGO OTPGRVEKGKKMAGHLGDERVTVOGLEI \\
\hline
\end{tabular}

Figure 4-9. The amino acid alignment of the $50 \mathrm{~S}$ ribosomal protein $\mathrm{L} 3(\mathrm{RplC})$ from a UT-490 (68)-Resistant $A$. baumannii strain (68R).

$\mathbf{6 8}^{\mathrm{R}}$ (Query) and wild-type control (Sbjct): the red color represents the site mutation in RplC. (Reprinted with permission. Siricilla, S., Mitachi, K., Yang, J., Eslamimehr, S., Lemieux, M. R., Meibohm, B., Ji, Y., and Kurosu, M. (2017). A new combination of a pleuromutilin derivative and doxycycline for treatment of multidrug-resistant Acinetobacter baumannii. J. Med. Chem. American Chemical Society. Article ASAP) 


\section{CHAPTER 5. CONCLUSIONS AND FUTURE DIRECTIONS}

\section{Conclusions}

The efforts to control and prevent TB are seriously threatened by the emergence of MDR, XDR and TDR strains of $M t b$. The current regimen to treat MDR- and XDR-TB takes several years. Treatment of TDR-TB requires completely novel therapeutic strategy. Drugs that shorten the treatment time of TB chemotherapy is extremely important. $M t b$ is known to survive by entering a non-replicating (dormant) state in the host. $M t b$ can exist in the dormant state for many years in the host, causing the latent infection. To discover drugs that kill $M t b$ in non-replicating state is believed to be a promising strategy to shorten the current TB chemotherapy. This dissertation discusses the development of novel antimycobacterial drug leads effective against both replicatingand non-replicating $M t b$ at low concentrations. These antimycobacterial agents summarized here have the potential to shorten the treatment for TB.

New antimycobacterial molecules that kill non-replicating $M t b$ were identified by screening libraries of synthetic natural products and their analogs (Figure 5-1). Screening of a 400-membered library of aurachin RE analogs resulted in the discovery of UT-317, $(R)$-20. UT-317 is a selective MenA inhibitor which ultimately inhibits biosynthesis of the electron carrier, vitamin $\mathrm{K}_{2}$. UT-317 exhibits an antimicrobial spectrum focused against $M t b$, and effectively kills both replicating and non-replicating $M t b$.

In our ongoing programs of discovery of new drug leads against bacterial membrane-associated proteins, novel fluorescent probes, Park's nucleotide- $N^{\varepsilon}-\mathrm{C}_{6}$-FITC (32, for MraY/MurX) and UDP-glucosamine- $\mathrm{C}_{6}$-FITC (46, for WecA) were developed. These probes enable us to perform medium- to high-throughput screening. Preliminary screening of a 50-membered library of capuramycin analogs against translocase I (MraY/MurX) and WecA identified a selective WecA inhibitor, UT-01320 (45) that kills both replicating and non-replicating $M t b$. WecA catalyzes the first step of mycolylarabinogalactan conjugate biosynthesis of Mtb cell wall. The efficacy of UT-01320 against non-replicating bacilli is especially important, suggesting that some cell wall biosynthesis is necessary to maintain viability of $M t b$ in a latent state.

Screening of a 50-membered library of pleuromutilin derivatives resulted in discovery of UT-800 (64) that displays a strong antimycobacterial activity. UT-800 is a first-in-class pleuromutilin analog that kills non-replicating $M t b$ under anaerobic conditions and exhibits efficacy against intracellular $M t b$.

\section{Future Directions}

It would be interesting to see the in vivo efficacy of the identified MenA inhibitors using the mouse infection models. A new MenA inhibitor UT-321 (structure not shown) has demonstrated effectiveness against Clostridium difficile in vivo. Thus, a 


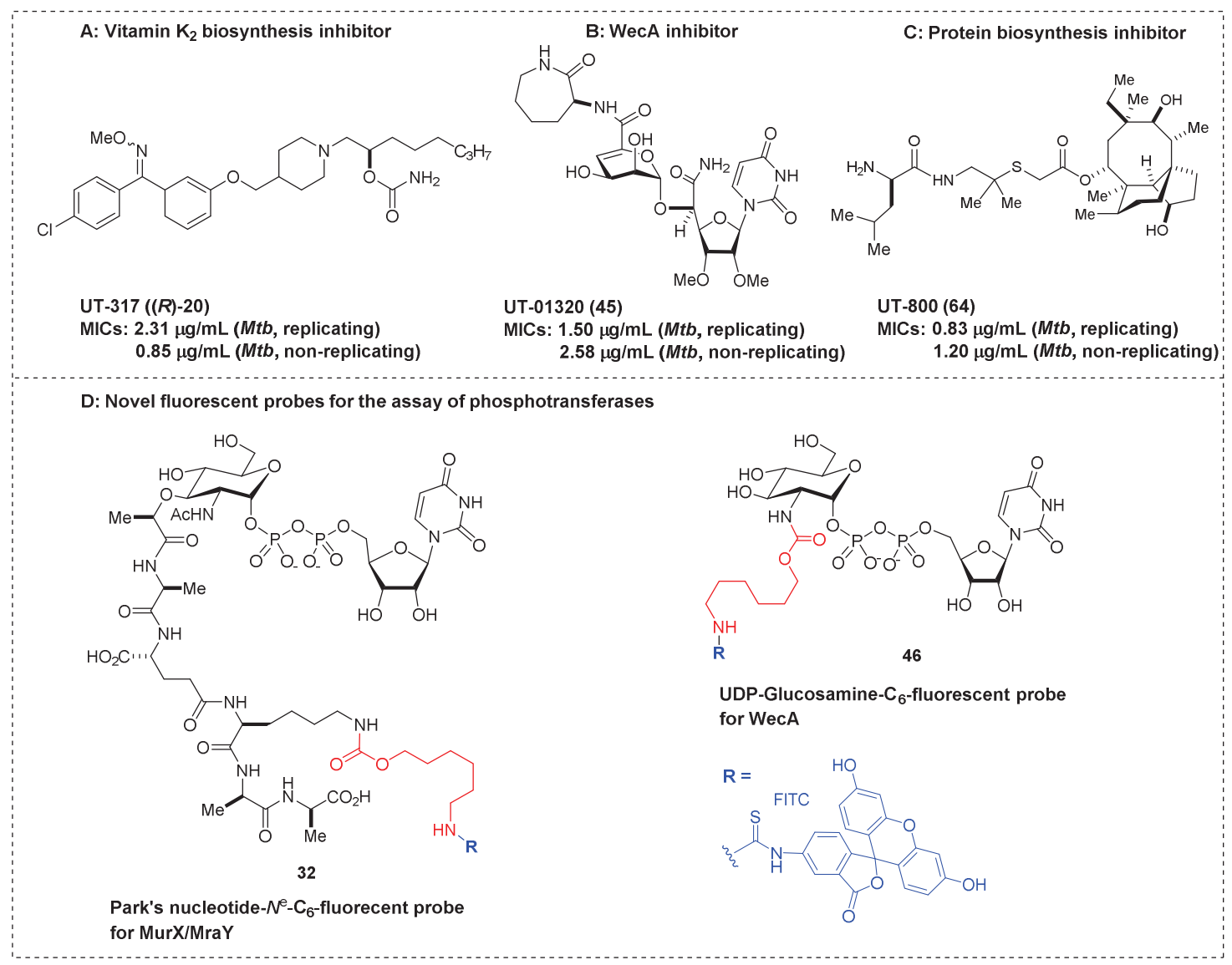

Figure 5-1. Drug leads identified against dormant $M t b$ and novel fluorescent probes for the assay of phosphotransferases. 
pharmacological proof-of-concept of a MenA inhibitor was demonstrated for the first time. Similarly, a MenA inhibitor having an appropriate ADMET profile is expected to demonstrate in vivo efficacy in the $M t b$ infection animal models.

UT-01320 (45) is the only selective WecA inhibitor identified to date. The in vitro activity, intracellular activity and narrow spectrum of activity towards $M t b$ demonstrated for 45 is excellent. UT-01320 did not exhibit toxicity in in vitro assays. In order to establish a pharmacological proof-of-concept, the PK/PD characteristics of $\mathbf{4 5}$ need to be studied. Our lab is currently optimizing the synthesis of $\mathbf{4 5}$ that enables us to generate over four gram-quantity as a pure form. WecA is required for the synthesis of mycolylarabinogalactan, an essential component of cell wall for $M t b$ to survive in macrophages. Genetic and microbiological studies of UT-01320 escape mutants will validate the unique mode of action of UT-01320 and WecA target for dormant Mtb.

UT-800 (64) has demonstrated its metabolic stability ( $\left.t_{1 / 2}>60 \mathrm{~min}\right)$. Our group has initiated the pharmacokinetics, oral bioavailability and in vivo toxicity studies of UT-800 using preclinical animal models. 


\section{CHAPTER 6. MATERIALS AND METHODS}

\section{General Information}

All antimycobacterial molecules were synthesized in our laboratory except for control compounds. The control compounds were obtained from sigma. All molecules were diluted with DMSO/ $\mathrm{H}_{2} \mathrm{O} / \mathrm{H}_{2} \mathrm{O}: \mathrm{PEG}(1: 2)$ mixture to be the concentration of 1 $\mathrm{mg} / 100 \mu \mathrm{L}$ (stock solution). Difco Middlebrook 7H10 agar, Middlebrook 7H9 broth, Tryptic soy agar, Tryptic soy broth, MOPS, tris(hydroxymethyl)aminomethane, $\beta$ mercaptoethanol, triton-X 100, CHAPS, DMSO, resazurin, methylene blue, tween 80, menaquinone $\left(\mathrm{VK}_{2}\right)$ were purchased from Sigma-Aldrich. ADC, OADC enrichment, MTT, EMEM and FBS were purchased from Fisher Scientific. Magnesium chloride and potassium chloride were obtained from VWR. E. coli circular DNA assay kit (L1020) for transcription / translation coupled assay was purchased from Promega Corporation.

\section{Bacterial Strains}

Mycobacterium tuberculosis $\left(\mathrm{H}_{37} \mathrm{Rv}, \mathrm{H}_{37} \mathrm{Rv} \mathrm{INHr}\right.$ and $\left.\mathrm{H}_{37} \mathrm{Rv} \mathrm{RFPr}\right)$ were obtained through BEI Resources, NIAID/NIH. Mycobacterium smegmatis (ATCC 607 and ATCC 14468), Mycobacterium bovis (BCG), Staphylococcus aureus (ATCC 25923, BAA 2094, BAA 44, and BAA 1683), Enterococcus faecium (ATCC 349), Enterococcus faecalis (ATCC 19433), Streptococcus pneumoniae (ATCC 6301), Streptococcus salivarius (ATCC 7073), Bacillus subtilis (ATCC 6051), Clostridium difficile (), Klebsielle pneumoniae (ATCC 8047), Pseudomonas aeruginosa (ATCC 27853), Acinetobacter baumannii (ATCC 19606), and Escherichia coli (ATCC 10798) were obtained from ATCC. E. coli B21 wecA strain was obtained from Dr. McNeil (Colorado State University).

\section{Bacterial Culture Conditions}

A single colony of each Mycobacterium strain $\left(\mathrm{H}_{37} \mathrm{Rv}, \mathrm{H}_{37} \mathrm{Rv} \mathrm{INHr}, \mathrm{H}_{37} \mathrm{Rv} \mathrm{RFPr}\right.$, M. smegmatis ATCC 607, ATCC 14468 and M. bovis (BCG)) was obtained on a Difco Middlebrook 7H10 nutrient agar enriched with 10\% oleic acid, albumin, dextrose and catalase (OADC for $M t b$ ), albumin, dextrose and catalase (ADC for M. smegmatis and $M$. bovis). Tryptic Soy agar was used for all other strains except $C$. difficile. A single colony of $C$. difficile was obtained on modified reinforced clostridial agar that was prepared anaerobically.

Seed cultures of each Mycobacterium strain were obtained in Middlebrook 7H9 broth enriched with OADC (for Mtb), ADC (for M. smegmatis and M. bovis). Tryptic Soy broth was used for all other strains except $C$. difficile. $C$. difficile was cultured in modified reinforced clostridial broth that was prepared anaerobically in an anerobic chamber under $10 \% \mathrm{H}_{2}, 5 \% \mathrm{CO}_{2}$, and $85 \% \mathrm{~N}_{2}$ conditions. Flasks containing each 
bacterial strain was incubated to mid-log phase in their respective culture media, in a shaking incubator at $37^{\circ} \mathrm{C}$ with a shaking speed of $200 \mathrm{rpm}$ and cultured to mid-log phase (Optical density - 0.5). The optical density was monitored at $600 \mathrm{~nm}$ using a microplate reader (Biotek Synergy XT). All experiments involving Mtb were performed in a BSL3 facility.

\section{Screening and Assay Development Procedures}

\section{Minimum Inhibitory Concentration Assays}

Microplate Alamar Blue Assay (MABA). These were performed according to the published protocol (134). Bacterial cultures at 0.5 optical density, was treated with serial dilutions of inhibitors in aerobic conditions and incubated at $37^{\circ} \mathrm{C}$ for 15 days for $M t b\left(\mathrm{H}_{37} \mathrm{Rv}, \mathrm{H}_{37} \mathrm{Rv} \mathrm{INHr}\right.$ and $\left.\mathrm{H}_{37} \mathrm{Rv} \mathrm{RFPr}\right)$ and $M$. bovis (BCG). Incubation time was 48h for M. smegmatis (ATCC 607and 14468). All other bacteria were incubated for $24 \mathrm{~h}$. After incubation, $20 \mu \mathrm{L}$ of resazurin (stock-0.02\%) was added and incubated on a shaking incubator at $37^{\circ} \mathrm{C}$ for $4 \mathrm{~h}$ for $\mathrm{Mtb}$ and $\mathrm{M}$. bovis. For all other bacteria $20 \mu \mathrm{L}$ was added from a $0.08 \%$ resazurin stock solution and left for $1 \mathrm{~h}$. The lowest concentration at which the color of resazurin was completely retained as blue was read as the $\mathrm{MIC}_{100}$ (Pink = Growth, Blue $=$ No growth). The absorbance measurements were also performed using a Biotek Synergy XT (Winooski, VT, USA), 96 well plate reader at $570 \mathrm{~nm}$ and $600 \mathrm{~nm}$. Assay plates for $C$. difficile was incubated for $24 \mathrm{~h}$ in the anaerobic conditions and the optical density was measured at the end of incubation and wells with no visible growth was considered MIC.

Luminescence-based Low-oxygen-recovery Assay (LORA). These assays were performed according to the reported procedures in the facility of Illinois TB Research Institute (47). In brief, $M t b \mathrm{H}_{37} \mathrm{Rv}$ cells were transformed by mixing at least $1 \mu \mathrm{g}$ of the purified plasmid, pFCA-luxAB and incubating at room temperature for $30 \mathrm{~min}$, followed by electroporation (216). Mtb pFCA-luxAB strain cultured was diluted in Middlebrook $7 \mathrm{H} 12$ broth, and sonicated for $15 \mathrm{~s}$. The cultures were diluted to obtain an $\mathrm{A}_{570}$ of 0.03 to 0.05 and 3,000 to 7,000 RLUs per $100 \mu \mathrm{l}$. Twofold serial dilutions of antimicrobial agents were prepared in black 96-well microtiter plates $(100 \mu \mathrm{l})$, and $100 \mu \mathrm{l}$ of the cell suspension was added. The microplate was placed under anaerobic conditions (oxygen concentration, less than $0.16 \%$ ) by using an Anoxomat model WS-8080 (MART Microbiology) and three cycles of evacuation and filling with a mixture of $10 \% \mathrm{H}_{2}, 5 \%$ $\mathrm{CO}_{2}$, and $85 \% \mathrm{~N}_{2}$. Incubation was continued for 10 days, and transferred to an ambient gaseous condition ( $5 \%$ CO2-enriched air) incubator for a $28 \mathrm{~h}$ "recovery." $100 \mu \mathrm{l}$ culture was transferred to white 96-well microtiter plates for determination of luminescence.

MIC on Nutrient Starved Mtb (Loebel Model Assay). $M t b \mathrm{H}_{37} \mathrm{Rv}$ was grown in conical flasks with air filter, containing Middlebrook 7H9 medium supplemented with 
OADC at $37^{\circ} \mathrm{C}$ according to the procedure established by Loebel. Exponentially growing bacilli at an $\mathrm{OD}_{600}$ of 0.2 were transferred to PBS supplemented with $0.025 \%$, Tween 80 and diluted to a final $\mathrm{OD}_{600}$ of $0.1 .50 \mathrm{ml}$ of this suspension was transferred into a conical flask and starved for 14 days at $37^{\circ} \mathrm{C}(137,187,217) .100 \mu \mathrm{L}$ of the culture was transferred to a 96 well plate and MIC experiment was performed according to the procedure described (vide supra).

\section{E. coli Growth Inhibitory Assays under Anaerobic Conditions}

E. coli was grown with inhibitor molecule $(5 \mu \mathrm{g} / \mathrm{mL})$ under anaerobic conditions in the modified $E$. coli growth media and the growth curve was monitored photometrically by reading the optical density at $600 \mathrm{~nm}(218,219)$ (Figure 6-1). E. coli growth rescue studies were performed by supplementing $\mathrm{VK}_{2}(50 \mu \mathrm{M})$.

\section{Oxygen Consumption Assays}

In sterile glass vial $1.5 \mathrm{~mL}$ of $M t b$ or $M$. smegmatis (grown to 0.5 OD value) was placed. Inhibitor molecule $(80 \mu \mathrm{L}$, total concentration ranged from $5-100 \mu \mathrm{g} / \mathrm{mL})$ and $0.01 \%$ methylene blue $(100 \mu \mathrm{L})$ was added into the culture solution. The culture vials were kept at $37^{\circ} \mathrm{C}$ for $22 \mathrm{~h}$. The effective concentration of the molecule to inhibit the electron transport systems was determined by comparing the color intensity with the control vial (no inhibitor) (Figure 6-2).

\section{Preparation of Membrane Fraction}

Mtb $\mathrm{H}_{37} \mathrm{Rv}$, M. smegmatis (ATCC 607), S. aureus (ATCC 25923) and E. coli (wild type; ATCC 10798 and BL21WecA) were grown to mid-log phase in their respective culture conditions described (vide supra) and the cells were harvested by centrifugation at $4{ }^{\circ} \mathrm{C}$ followed by washing with $0.9 \%$ saline solution (thrice) through centrifugation. $\sim 5 \mathrm{~g}$ of pellet (wet weight) was collected. The washed cell pellets were suspended in homogenization buffer (containing $50 \mathrm{mM}$ MOPS of $\mathrm{pH}=8,10 \mathrm{mM} \mathrm{MgCl}_{2}$ and $5 \mathrm{mM} 2$-mercaptoethanol) and disrupted by probe sonication on ice (10 cycles of $60 \mathrm{~s}$ on and $90 \mathrm{~s}$ off). The resulting suspension was centrifuged at $1,000 \times \mathrm{g}$ for $10 \mathrm{~min}$ at $4{ }^{\circ} \mathrm{C}$ to remove unbroken cells. The supernatant was centrifuged at $25,000 \times \mathrm{g}$ for $40 \mathrm{~min}$ at 4 ${ }^{\circ} \mathrm{C}$ (3 to 4 times). All pellets in each tube were pooled and a second sonication was performed (10 cycles of 60 s on and 270 s off). The lysate was centrifuged once at 25,000 $\times \mathrm{g}$ for $1 \mathrm{~h}$ and the supernatant was subjected to ultracentrifugation at $60,000 \times \mathrm{g}$ for $1 \mathrm{~h}$ at 4 ${ }^{\circ} \mathrm{C}$. The supernatant was discarded and the membrane fraction containing MenA, MurX, and WecA enzymes (P-60) was suspended in the TRIS-HCl buffer ( $\mathrm{pH} 7.5$, containing 2mercaptoethanol) (160,220). Total protein concentrations are about $8 \sim 10 \mathrm{mg} / \mathrm{mL}(221)$. Aliquots were stored in eppendorf tubes at $-80^{\circ} \mathrm{C}$. 
A

Growth Media:

Pancreatic Digest of Casein, $20.0 \mathrm{~g}$

Yeast Extract, $10.0 \mathrm{~g}$

Resazurin, $0.001 \mathrm{~g}$

Calcium Chloride, anhydrous, $0.008 \mathrm{~g}$

Magnesium Sulfate, anhydrous, $0.008 \mathrm{~g}$

Potassium Phosphate, monobasic, $0.04 \mathrm{~g}$

Potassium Phosphate, dibasic, $0.04 \mathrm{~g}$

Sodium Chloride, $0.08 \mathrm{~g}$

Sodium Bicarbonate, $0.4 \mathrm{~g}$

Hemin (0.1\% Soln), $5.0 \mathrm{~mL}$

L-Cysteine, $0.5 \mathrm{~g}$

Distilled Water, $1000.0 \mathrm{~mL}$
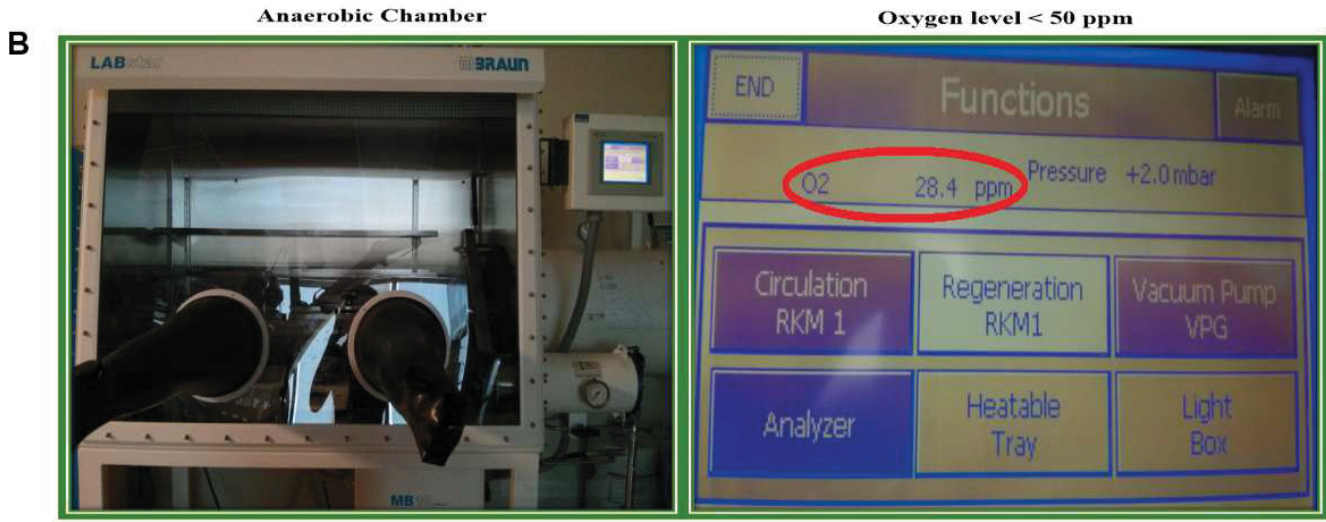

Figure 6-1. $\quad E$. coli growth inhibitory assays under anaerobic conditions.

A) E. coli was grown under anaerobic conditions using the growth media. B) Anaerobic conditions were maintained by using a mBraun LABstar anaerobe chamber and the $\mathrm{O}_{2}$ levels were maintained below 50 ppm.

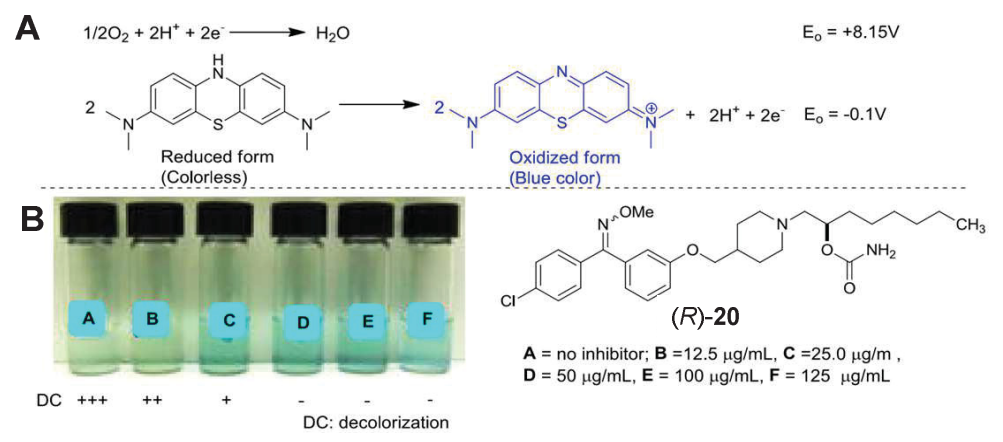

Figure 6-2. Oxygen consumption assays.

A) Oxidized form of methylene blue is blue in color while the reduced form is colorless.

B) Color intensity of methylene blue in vials with varying concentrations of inhibitor was monitored. 


\section{MenA Enzyme Inhibitory Assay (IC50)}

The substrate 1,4-dihydroxy-2-napthanoic acid (DHNA) (500 $\mu \mathrm{M} ; 20 \mu \mathrm{L}), \mathrm{MgCl}_{2}$ $(5 \mu \mathrm{M} ; 20 \mu \mathrm{L})$; CHAPS $(0.1 \% ; 20 \mu \mathrm{l})$, Tris-buffer $(\mathrm{pH}=8 ; 20 \mu \mathrm{L})$, membrane fraction containing Men A $(100 \mu \mathrm{l})$, inhibitors $(0-60 \mu \mathrm{g} / \mathrm{mL}$ of DMSO) and farnesyl pyro phosphate $(200 \mu \mathrm{M}, 40 \mu \mathrm{L})$ were mixed together and incubated for $2 \mathrm{~h}$ at $37^{\circ} \mathrm{C}$. After the incubation, the reaction mixture was quenched with $0.1 \mathrm{M} \mathrm{AcOH}$ in $\mathrm{MeOH}$ and the reaction mixture was extracted with hexanes (thrice). The organic portions were then concentrated and diluted with $\mathrm{MeOH}(300 \mu \mathrm{L})$. From each set of enzymatic reaction mixture $20 \mu \mathrm{l}$ was injected into the HPLC $\left(\mathrm{CH}_{3} \mathrm{CN}: 0.05 \%\right.$ TFA in $\mathrm{H} 2 \mathrm{O}=90: 10$, UV: 325 $\mathrm{nm}$, flow rate: $1.5 \mathrm{~mL} / \mathrm{min}$ ) and the area of the peak for DMMK was quantified to obtain the $\mathrm{IC}_{50}$ value for different inhibitor molecules (Figure 6-3).

\section{MurX Assay}

MurX assay substrates, Park's nucleotide- ${ }^{\varepsilon}{ }^{\varepsilon}$-dansylthiourea (29), Park's nucleotide- ${ }^{\varepsilon}$-FITC (32), and neryl phosphate (35), were chemically synthesized according to the reported procedures $(141,145,155,156,183) .29$ or 32 (2 mM stock solution; $3.75 \mu \mathrm{L}(75 \mu \mathrm{M})), \mathrm{MgCl}_{2}(0.5 \mathrm{M} ; 10 \mu \mathrm{L}(50 \mathrm{mM})), \mathrm{KCl}(2 \mathrm{M}, 10 \mu \mathrm{L}(200$ $\mathrm{mM})$ ), triton X100 $(0.5 \% ; 11.25 \mu \mathrm{L})$, tris-buffer $(\mathrm{pH}=8 ; 50 \mathrm{mM}, 2.5 \mu \mathrm{L})$, neryl phosphate $(10 \mathrm{mM}, 45 \mu \mathrm{L})$, and inhibitor $(0-100 \mu \mathrm{M}$, in DMSO $(2.5 \mu \mathrm{L}))$ were placed in a $500 \mu \mathrm{L}$ Eppendorf tube. To a stirred reaction mixture, P-60 (15 $\mu \mathrm{l})$ was added (the total volume of the reaction mixture: $100 \mu \mathrm{L}$ ). The reaction mixture was incubated for $1 \mathrm{~h}$ at room temperature $\left(26^{\circ} \mathrm{C}\right)$, and quenched with $\mathrm{CHCl}_{3}(200 \mu \mathrm{L})$. Two phases were mixed via vortex and centrifuged at $25,000 \times \mathrm{g}$ for $10 \mathrm{~min}$. The upper aqueous phase was assayed via reverse-phase HPLC. The water phase (10 $\mu \mathrm{l})$ was injected into HPLC (solvent: $\mathrm{CH}_{3} \mathrm{CN}$ : $0.05 \mathrm{M}$ aq. $\mathrm{NH}_{4} \mathrm{HCO}_{3}=25: 75$, UV: $350 \mathrm{~nm}$, flow rate: $0.5 \mathrm{~mL} / \mathrm{min}$, Column: Kinetex $5 \mathrm{u} \mathrm{C8} 100 \AA, 150 \times 4.60 \mathrm{~mm}$ ), and the area of the peak for lipid I-neryl derivative was quantified to obtain the $\mathrm{IC}_{50}$ value. The $\mathrm{IC}_{50}$ values were calculated from plots of the percent product inhibition versus the inhibitor concentration.

The MurX assays developed here were tolerated to $2.5 \%$ of DMSO concentrations in total volume of the reaction solution $(100 \mu \mathrm{L})$. The maximum tolerated concentration for DMSO in MurX-catalyzed neryl lipid I-N $\mathrm{N}^{\varepsilon}$-dansylthiourea has not been determined.

\section{WecA Assay}

UDP-Glucosamine-C 6 -FITC [2 mM stock solution; $0.56 \mu \mathrm{L}(22.5 \mu \mathrm{M})], \mathrm{MgCl}_{2}$ $[0.5 \mathrm{M} ; 4 \mu \mathrm{L}(40 \mathrm{mM})]$, CHAPS $(5 \% ; 10 \mu \mathrm{L}(1 \%)), \beta$-mercaptoethanol [50 mM; $5 \mu \mathrm{L}$ $(5 \mathrm{mM})]$, tris-buffer $(\mathrm{pH}=8.0 ; 50 \mathrm{mM}, 9.74 \mu \mathrm{L})$, decaprenyl phosphate dissolved in Tris$\mathrm{HCl}(50 \mathrm{mM}, \mathrm{pH}$ 8): PEG: DMSO (5: 1: 2) [4 mM, $1.7 \mu \mathrm{L}(140 \mu \mathrm{M})$ ], and inhibitor [0$100 \mu \mathrm{M}$, in DMSO $(1 \mu \mathrm{L})]$ were place in a $1.5 \mathrm{~mL}$ Eppendorf tube. P-60 $(18 \mu \mathrm{L})$ was added (the total volume of the reaction mixture: $50 \mu \mathrm{L}$ ) to the reaction mixture. The 

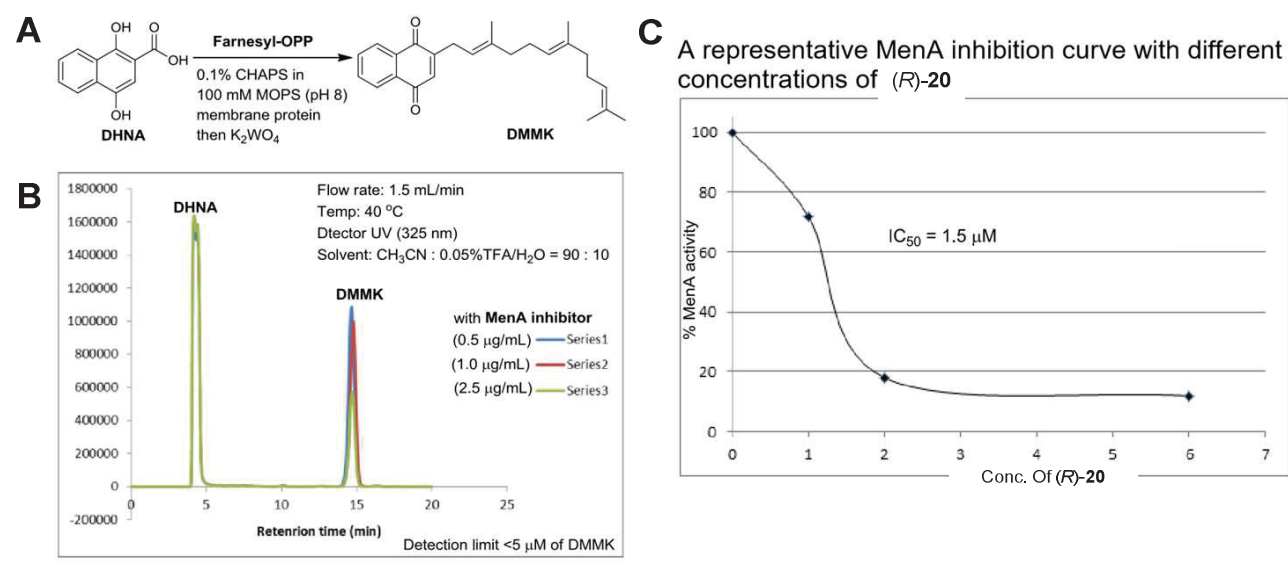
concentrations of $(R)-20$

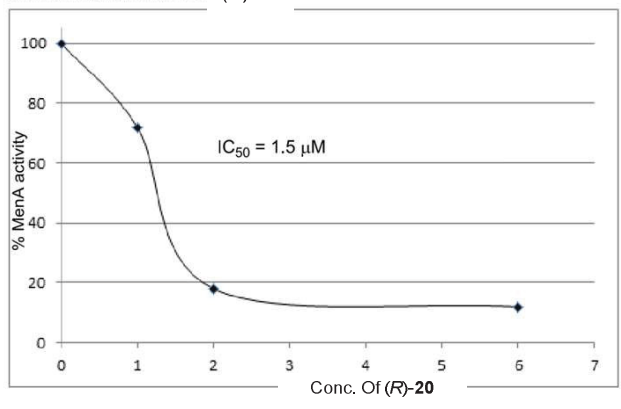

Figure 6-3. MenA assay.

A) MenA assay conditions. B) HPLC chromatogram of MenA assay depicting the retention times and concentration dependent change in peak area of substrate (DHNA) and MenA enzyme product (DMMK). C) A representative MenA inhibition curve with different concentrations of $(R)-\mathbf{2 0}$. 
reaction mixture was incubated for $2 \mathrm{~h}$ at $37^{\circ} \mathrm{C}$, and quenched with n-butanol $(150 \mu \mathrm{L})$. Two phases were mixed via vortex and centrifuged at $10,000 \times \mathrm{g}$ for $3 \mathrm{~min}$. The upper butanol phase was assayed via reverse-phase HPLC or UV-vis BioTek Synergy HT plate reader. For HPLC analyses, the butanol phase $(25 \mu \mathrm{L})$ was injected into HPLC (solvent: $\mathrm{CH}_{3} \mathrm{OH}: 0.05 \mathrm{M}$ aq. $\mathrm{NH}_{4} \mathrm{HCO}_{3}=85: 15$, UV: $485 \mathrm{~nm}$, flow rate: $0.5 \mathrm{~mL} / \mathrm{min}$, Column: Kinetex $5 \mu \mathrm{C} 8100 \AA \AA, 150 \times 4.60 \mathrm{~mm}$ ); the area of the peak for decaprenyl-P-PGlucosamine- $\mathrm{C}_{6}$-FITC was quantified to obtain the $\mathrm{IC}_{50}$ value. For the UV-visbased assay, the n-butanol phase $(20 \mu \mathrm{L})$ was transferred to a 384 well black plate and fluorescence was measured at an excitation of $485 \mathrm{~nm}$ and emission of $528 \mathrm{~nm}$. The $\mathrm{IC}_{50}$ values were calculated from plots of the percent product inhibition versus the inhibitor concentration.

\section{Kill-curve Graph: Determination of Colony Forming Units per Milliliter}

Mtb cultures at mid-log phase $(\mathrm{OD}=0.5)$ were diluted to $\mathrm{OD}=0.25$ and treated with inhibitor molecules at MIC, x2 MIC and x4 MIC. Each culture well was diluted 10, 100,1000 and 10,000 fold every $24 \mathrm{~h}$ and $20 \mu \mathrm{L}$ from each dilution was plated on $7 \mathrm{H} 10$ agar plates supplemented with OADC enrichment. Plates were incubated for 15 days in a static incubator at $37^{\circ} \mathrm{C}$ and colonies were counted.

\section{Determination of Cytotoxicity $\left(\mathrm{IC}_{50}\right)$ in Vero and $\mathrm{HepG}_{2}$ Cells}

Cytotoxicity assays were performed using Vero monkey kidney (ATCC CCL-81) and $\mathrm{HepG}_{2}$ human hepatoblastoma cell (ATCC HB-8065) lines. Vero or HepG 2 cells were subcultured in $75 \mathrm{~cm}^{2}$ cell culture flasks using ATCC-formulated eagle's minimum essential medium (EMEM) containing 10\% fetal bovine serum and penicillinstreptomycin at a subcultivation ratio of $1: 3$.

Cells were transferred to a 96 well cell culture plate and serially diluted aliquots of each test compound at concentrations ranging from 1-25 $\mathrm{x}$ of the MIC for $M t b \mathrm{H}_{37} \mathrm{Rv}$ were added to the cells. Control compounds with known toxicity such as rifampicin, $\mathrm{INH}$, tunicamycin or atovaquone were included on each plate. The plates were incubated for 72 $\mathrm{h}$ and cytopathic effects were determined via the MTT assay. Cell viability was assessed on the basis of cellular conversion of MTT into a purple formazan product (Figure 6-4). The absorbance of the colored formazan product was measured at $570 \mathrm{~nm}$ by BioTek Synergy HT Spectrophotometer. Selectivity index $\left(\mathrm{SI}=\mathrm{IC}_{50} / \mathrm{MIC}\right)$ of SI $>10$ was considered to be significant in this program.

\section{Kinetic Parameter Evaluation}

MurX/MraY Activity Assay. Evaluation of kinetic parameters were performed through M. smegmatis MurX/MraY-catalyzed lipid I synthesis. The MurX/MraY activity 

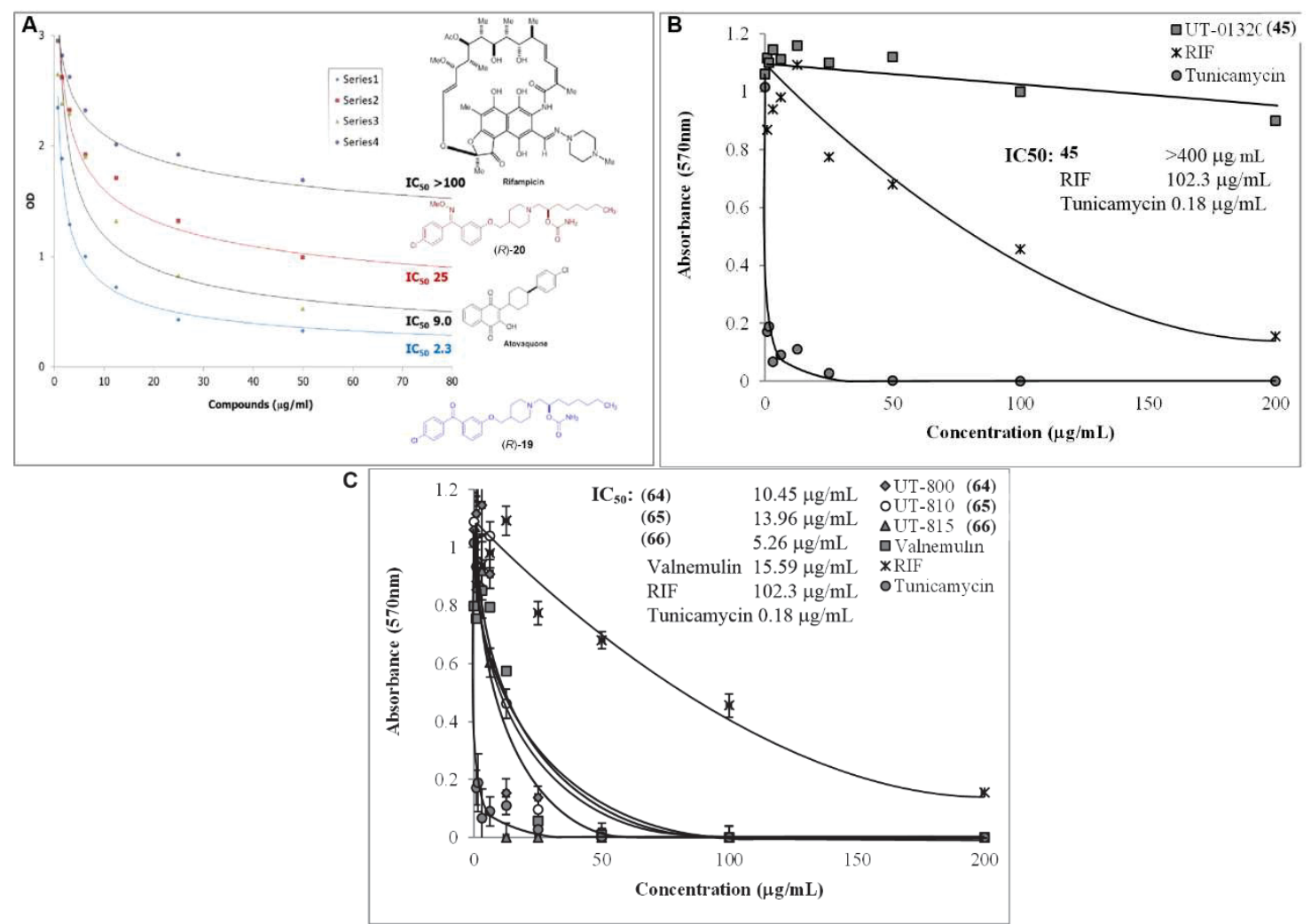

Figure 6-4. Cytotoxicities (IC 50 ) of selected compounds with activity against $M t b$ $\mathrm{H}_{37} \mathrm{Rv}$ in Vero monkey kidney cells and $\mathrm{HepG}_{2}$ cells.

A) Cytotoxicities $\left(\mathrm{IC}_{50}\right)$ of $(R)-\mathbf{1 9},(R)-\mathbf{2 0}$, atovaquone, and rifampin in $\mathrm{HepG}_{2}$ cells. B) Cytotoxicities ( $\left.\mathrm{IC}_{50}\right)$ of UT-01320 (45), tunicamycin and rifampicin in Vero cells. C) Cytotoxicities ( $\mathrm{IC}_{50}$ ) of UT-800 (64), UT-810 (65), UT-815 (66), valnemulin, tunicamycin and rifampin in Vero cells. All plotted data are an average of three experiments. 
was assayed with various concentrations of Park's nucleotide- $\mathrm{N}^{\varepsilon}$-dansylthiourea while keeping the concentration of Neryl $\mathrm{PO}_{4}$ constant $(4.5 \mathrm{mM}) . \mathrm{Km}$ and Vmax were determined by Michaelis-Menten enzyme kinetics. The correlation (Michaelis-Menten plot) between the concentrations of Park's nucleotide- $\mathrm{N}^{\varepsilon}$-dansylthiourea ( $\mathrm{x}$ axis) and rate (V) of lipid I formation (y axis) was obtained using GraphPad Prism Software $(174,222)$.

WecA Activity Assay. Evaluation of kinetic parameters was performed through E. coli (B21WecA) P-60-catalyzed WecA synthesis. The WecA activity was assayed with various concentrations of UDP-GlcNAc- $\mathrm{C}_{6}$-FITC while keeping the concentration of undecaprenyl $\mathrm{PO}_{4}$ constant $(140 \mu \mathrm{M})$. Km and Vmax were determined by MichaelisMenten enzyme kinetics. The correlation (Michaelis-Menten plot) between the concentrations of UDP-Glucosamine- $\mathrm{C}_{6}$-FITC ( $\mathrm{x}$ axis) and rate $(\mathrm{V})$ of decaprenylGlucosamine- $\mathrm{C}_{6}$-FITC (y axis) formation was obtained using GraphPad Prism Software.

\section{Preparation of Polyprenyl Phosphates for MurX and WecA Assays}

Decaprenyl phosphate $\left(\mathrm{C}_{50}-\mathrm{P}\right)$ and undecaprenyl phosphate $\left(\mathrm{C}_{55}-\mathrm{P}\right)$ were prepared as follows: Leaves of Magnolia kobus, (Rosaceae) were collected in the Botanical Garden of Polish Academy of Sciences, Powsin, dried in open air, pulped using mortar and pestle and extracted with acetone/hexane $(1: 1, \mathrm{v} / \mathrm{v})$ for $48 \mathrm{~h}$ at $\mathrm{r}$.t. After filtration and evaporation of the solvents, the crude mixture of lipids containing polyprenols was dissolved in hexane and purified by silica gel chromatography (hexanes to $1: 1$ hexanes/Et $2 \mathrm{O})(223,224)$. The crude polyprenol mixture was purified by $\mathrm{C}_{18}$-silica gel chromatography (LiChroprep RP-18, 40-63 $\mu \mathrm{m}$, Merck, solvent: MeOH to acetone: $\mathrm{MeOH}=10 / 1)$. Fractions containing pure decaprenol and undecaprenol were pooled, evaporated, dissolved in hexane and stored at $-20^{\circ} \mathrm{C}$. Polyprenyl phosphates were synthesized via the procedures reported by Danilov et al. (225). Stirred solution of decaprenol in $\mathrm{CHCl}_{3}$ was added $\mathrm{Cl}_{3} \mathrm{CN}$ and $\mathrm{nBu}_{4} \mathrm{NH}_{2} \mathrm{PO}_{4}$. After 30 min at room temperature, all volatiles were evaporated and the residue was dissolved in THF. Aqueous ammonia was added to the mixture and kept overnight at $4{ }^{\circ} \mathrm{C}$. The supernatant was collected, evaporated and dissolved in $\mathrm{CHCl}_{3} / \mathrm{MeOH}(2: 1, \mathrm{v} / \mathrm{v})$ containing 3\% of water and loaded onto DEAE-Sephadex A-25 column. Unreacted decaprenol was eluted with $\mathrm{CHCl}_{3} / \mathrm{MeOH}(2: 1)$ and the decaprenyl phosphate was eluted with ammonium acetate $(0-100 \mathrm{mM})$ in $\mathrm{CHCl}_{3} / \mathrm{MeOH}(2: 1)$. Purified decaprenyl phosphate was dissolved in $\mathrm{CHCl}_{3} / \mathrm{MeOH}(2: 1)$ containing $3 \%$ of $\mathrm{NH}_{3}$ and stored at $-20{ }^{\circ} \mathrm{C}$. Undecaprenyl phosphate was synthesized by the same method described for decaprenyl phosphate.

\section{Synergistic Effect of UT-01320 (45) with SQ 641 (37) or Capuramycin (36)}

The synergistic or antagonistic activities of MurX inhibitors (capuramycin or SQ 641) and UT-01320 were assessed in vitro via micro dilution broth checkerboard 
technique $(189,226)$ (Figure 6-5). The FIC index was calculated to according to the following equation. $\mathrm{FIC}=\mathrm{FIC}_{\mathrm{A}}+\mathrm{FIC}_{\mathrm{B}}=\mathrm{C}_{\mathrm{A}} / \mathrm{MIC}_{\mathrm{A}}+\mathrm{C}_{\mathrm{B}} / \mathrm{MIC}_{\mathrm{B}}$ where, $\mathrm{MIC}_{\mathrm{A}}$ and $\mathrm{MIC}_{\mathrm{B}}$ : $\mathrm{MIC}$ of drugs $\mathrm{A}$ and $\mathrm{B}, \mathrm{C}_{\mathrm{A}}$ and $\mathrm{C}_{\mathrm{B}}=$ concentrations of drugs $\mathrm{A}$ and $\mathrm{B}$ used in combination. In these interaction studies, $\Sigma F I C$ of less than 0.5 represents synergistic activity (Table 6-1).

\section{Killing Effect against Intracellular Mtb}

J774A. 1 cells were seeded at $2.5 \times 10^{5}$ cells/well in 24 -well dishes or $1 \times 10^{5}$ cells/well in 8-well chamber slides and incubated overnight at $37^{\circ} \mathrm{C}$ in DMEM. A transformant $M t b$ CDC1551 expressing tdTomato was grown in 7H9 Middlebrook medium supplemented with OADC. The $M t b$ cells were harvested at an optical density of 0.5 , washed and re-suspended in saline. J774A.1 cells were maintained in cell culture medium and were infected by $M t b\left(10^{6}\right.$ bacteria in $0.2 \mathrm{~mL}$ of media): a multiplicity of infection (167) of $\approx 10$ (bacteria/cell). The extracellular bacteria were removed by washing with PBS. The infected macrophages were treated with antibacterial agents at $x 2$ and $\mathrm{x} 4 \mathrm{MIC}$ concentrations and the relative intensity of the fluorescence was measured [emission wavelength $(581 \mathrm{~nm})$ ] via UV-vis spectroscopy in 24,48 , and $72 \mathrm{~h}$ for inhibition of intracellular bacterial growth. Surviving $M t b$ cells were confirmed by CFU method (227).

\section{Microsomal Stability}

Pooled Sprague-Dawley rat liver microsomes were purchased from Corning Life Sciences (Oneonta, NY, USA). Microsomes $((20 \mathrm{mg} / \mathrm{mL})$ were thawed on ice and diluted using phosphate buffer $(100 \mathrm{mM}, \mathrm{pH}: 7.4)$, resulting in a protein concentration of 1 $\mathrm{mg} / \mathrm{mL}$. Stock solutions $(10 \mathrm{mg} / \mathrm{L})$ of UT-800, UT-810, UT-815, valnemulin and verapamil (positive control) were prepared in DMSO (50\%). A final concentration of 500 $\mathrm{ng} / \mathrm{mL}$ was used for incubation with microsomes. NADPH (final concentration: $1 \mathrm{mM}$ ) was used as a co-factor. All the above solutions except NADPH were added to individual wells (12-well) in triplicate and were allowed to equilibrate for $5 \mathrm{~min}$ at $37^{\circ} \mathrm{C}$. $\mathrm{NADPH}$ was then added. $50 \mu \mathrm{L}$ aliquots in triplicate were drawn from the incubation mixture at $0,5,10,20,30,45$ and $60 \mathrm{~min}$ and immediately the reaction was quenched by addition of ice-cold methanol (4 volumes) (228). The samples containing methanol was lyophilized to remove all volatiles. The residue was dissolved in $1 \mathrm{~N} \mathrm{HCl}$ aq. $(10 \mu \mathrm{L})$ and $\mathrm{MeOH}(40 \mu \mathrm{L})$. The resulting solution $(20 \mu \mathrm{L})$ was injected to LC-MS. MS solvent 90:10 acetonitrile $/ 0.05 \%$ formic acid in water. Flow rate: $0.5 \mathrm{~mL} / \mathrm{min}$.

\section{Gene Analyses of Resistant Strain of A. baumannii against UT-490 (68)}

Spontaneous resistant mutants of A. baumannii ATCC 19606 with decreased sensitivity towards $\mathbf{6 8}$ were isolated by sub-culturing bacteria on agar plates with

concentrations of UT-490 at MIC and above. Resistant mutants were isolated at $16 \mathrm{x}$ 
A SQ $641(\mu \mathrm{g} / \mathrm{mL})$

\begin{tabular}{|c|c|c|c|c|c|c|c|c|}
\hline 6.24 & 4.05 & 4.11 & 4.21 & 4.43 & 4.86 & 5 & 7.44 & 10.88 \\
\hline 3.12 & 2.05 & 2.11 & 2.21 & 2.43 & 2.86 & 3 & 5.44 & 8.88 \\
\hline 1.56 (MIC) & 1.05 & 1.11 & 1.21 & 1.43 & 1.86 & 2 & 4.44 & 7.88 \\
\hline 0.78 & 0.55 & 0.61 & 0.71 & 0.93 & 1.36 & 1.50 & 3.94 & 7.38 \\
\hline 0.391 & 0.30 & 0.36 & 0.46 & 0.68 & 1.11 & 1.25 & 3.69 & 7.13 \\
\hline 0.2 & 0.18 & 0.23 & 0.34 & 0.56 & 0.99 & 1.13 & 3.57 & 7.01 \\
\hline 0.1 & 0.12 & 0.17 & 0.28 & 0.49 & 0.92 & 1.06 & 3.50 & 6.94 \\
\hline \multirow[t]{2}{*}{0.05} & 0.09 & 0.14 & 0.26 & 0.46 & 0.89 & 1.03 & 3.47 & 6.91 \\
\hline & 0.08 & 0.15 & 0.32 & 0.645 & 1.29 & 1.50 (MIC) & 5.16 & 10.32 \\
\hline
\end{tabular}

B Capuramycin $(\mu \mathrm{g} / \mathrm{mL})$

\begin{tabular}{|c|c|c|c|c|c|c|c|c|}
\hline 50 & 4.05 & 4.11 & 4.21 & 4.43 & 4.86 & 5 & 7.44 & 10.88 \\
\hline 25 & 2.05 & 2.11 & 2.21 & 2.43 & 2.86 & 3 & 5.44 & 8.88 \\
\hline 12.5 (MIC) & 1.05 & 1.11 & 1.21 & 1.43 & 1.86 & 2 & 4.44 & 7.88 \\
\hline 6.25 & 0.55 & 0.61 & 0.71 & 0.93 & 1.36 & 1.50 & 3.94 & 7.38 \\
\hline 3.125 & 0.30 & 0.36 & 0.46 & 0.68 & 0.11 & 1.25 & 3.69 & 7.13 \\
\hline 1.56 & 0.18 & 0.23 & 0.34 & 0.56 & 0.99 & 1.13 & 3.57 & 7.01 \\
\hline 0.78 & 0.12 & 0.17 & 0.28 & 0.49 & 0.92 & 1.06 & 3.50 & 6.94 \\
\hline \multirow[t]{2}{*}{0.391} & 0.08 & 0.14 & 0.24 & 0.46 & 0.89 & 1.03 & 3.47 & 6.91 \\
\hline & 0.08 & 0.16 & 0.32 & 0.645 & 1.29 & 1.50 (MIC) & 5.16 & 10.32 \\
\hline
\end{tabular}

Figure 6-5. Checkerboard combinations of UT-01320 with SQ641 or Capuramycin.

A) Checkerboard combinations of SQ641 (MIC $=1.56 \mu \mathrm{g} / \mathrm{mL})$ and UT-01320 (MIC = $1.50 \mu \mathrm{g} / \mathrm{mL})$. B) Checkerboard combinations of Capuramycin ( $\mathrm{MIC}=12.5 \mu \mathrm{g} / \mathrm{mL})$ and

UT $320(\mathrm{MIC}=1.50 \mu \mathrm{g} / \mathrm{mL})$. Shaded wells indicate growth and white wells indicate no growth. Each well shows calculated $\Sigma$ FIC values. 
Table 6-1. $\quad$ EFIC index for the wells at growth - no growth interface.

\begin{tabular}{|c|c|c|c|}
\hline Drug & MIC (Alone $-\mu \mathrm{g} / \mathrm{mL}$ ) & $C_{A}$ and $C_{B}(\mu g / m L)$ & EFIC \\
\hline SQ 641 (Drug A) & 1.56 & 0.1 & 0.12 \\
\hline UT-01320 (Drug B) & 1.50 & 0.08 & \\
\hline SQ 641 (Drug A) & 1.56 & 0.1 & 0.17 \\
\hline UT-01320 (Drug B) & 1.50 & 0.16 & \\
\hline SQ 641 (Drug A) & 1.56 & 0.1 & 0.28 \\
\hline UT-01320 (Drug B) & 1.50 & 0.32 & \\
\hline SQ 641 (Drug A) & 1.56 & 0.1 & 0.49 \\
\hline UT-01320 (Drug B) & 1.50 & 0.645 & \\
\hline SQ 641 (Drug A) & 1.56 & 0.1 & 0.92 \\
\hline UT-01320 (Drug B) & 1.50 & 1.29 & \\
\hline SQ 641 (Drug A) & 1.56 & 0.05 & 1.03 \\
\hline UT-01320 (Drug B) & 1.50 & 1.50 & \\
\hline Capuramycin (Drug A) & 12.5 & 1.563 & 0.18 \\
\hline UT-01320 (Drug B) & 1.50 & 0.08 & \\
\hline Capuramycin (Drug A) & 12.5 & 1.563 & 0.23 \\
\hline UT-01320 (Drug B) & 1.50 & 0.16 & \\
\hline Capuramycin (Drug A) & 12.5 & 0.78 & 0.28 \\
\hline UT-01320 (Drug B) & 1.50 & 0.32 & \\
\hline Capuramycin (Drug A) & 12.5 & 0.78 & 0.49 \\
\hline UT-01320 (Drug B) & 1.50 & 0.645 & \\
\hline Capuramycin (Drug A) & 12.5 & 0.78 & 0.92 \\
\hline UT-01320 (Drug B) & 1.50 & 1.29 & \\
\hline Capuramycin (Drug A) & 12.5 & 0.391 & 1.03 \\
\hline UT-01320 (Drug B) & 1.50 & 1.50 & \\
\hline
\end{tabular}

NOTE: $\mathrm{C}_{\mathrm{A}}$ and $\mathrm{C}_{\mathrm{B}}$ are concentrations of Drug $\mathrm{A}$ and Drug $\mathrm{B}$ in combination. 
MIC of 68. Spontaneous resistant mutants with decreased sensitivity against rifampicin were isolated at $32 \times$ MIC of rifampicin (control). The chromosomal DNAs from the resistant mutant $\left(\mathbf{6 8}^{\mathrm{R}}\right)$ and wild-type Acinetobacter baumannii ATCC19606 were

isolated. The $\mathrm{rplC}$ gene fragment was amplified using $A$. baumannii $r p l C$ specific primers (AbrplCupPrimer 5' CTTTGGGTTA AGGCTTTCGG 3' and Abrplcdn

5'CAACAGCAGAGCCG GAAACAG 3'), purified, and DNA sequenced $(214,215)$. The DNA sequencing result was used to blast against $r p l C$ DNA sequence of $A$. baumannii in NIH Genome database (Figure 6-6). 
A TTAACAAAGATGCGCGTGACCAGTACGAAATCCGCACCTACAAACGTTTGATCGACATCG TTCAACCTACAGATAAAACTGTTGATGCATTGATGAAGTTAGATCTTGCAGCTGGTGTTG ATGTTCAGATCGCTTTGGGTTAAGGCTTTCGGTTAATTAACGATTAGTTAATTAGGCCGC TTTTTTAGAGGTTTATGCACATGGCTATTGGTTTAGTCGGTCGCAAATGTGGTATGACTC GCATCTTTACAGATGCTGGTGTTTCTGTACCTGTTACAGTCATCGAAGTCGATCCAAACC GCATTACGCAАATCAАAАCACTTGAAACTGATGGTTATCAAGCTGTTCAAGTAACTACTG GCGAACGTCGCGAGTCTCGCGTAACTAACGCTCAAAAAGGTCACTTCGCTAAAGCGGGTG TTGCTGCTGGTCGTTTAGTTAAAGAGTTTCGTGTTACTGAAGCTGAGCTTGAAGGCCGTG AAGTTGGCGGTACTATTGGCGTTGATTTGTTCACAGTTGGTCAAATTGTTGACGTAACTG GTCAATCAAAAGGTAAAGGTTTCCAAGGTGGTGTTAAACGTTGGAATTTCCGTACCCAAG ATGCTACTCACGGTAACTCTGTTTCTCACCGTGTTTTAGGTTCTACAGGTCAAAACCAAA CTCCTGGACGCGTGTTCAAAGGCAAAAAAATGGCTGGTCACTTAGGTGATGAACGCGTAA CAGTACAAGGTCTTGAAATCGTATCTGTTGACACTGAACGTTCAGTTTTGGTTGTTAAGG GTGCAATTCCTGGTGCAACTGGCGGTGACGTTATCGTACGTCCTACCATCAAGGCCTGAG GGGAAATACCGTGAATTTAAAAACTGTTTCCGGCTCTGCTGTTGAATTGTCTGAAGTAGC TTTCGGACGTGAATTTAACGAAGCTCTTGTACACCAAGTTGTTACTGCTTACTTAGCAGG TGGTCGTCAAGGTACTCGTGCTCACAAATCACGTGCAGACGTTTCTGGCGGTGGTAAAAA ACCATTCCGTCAAAAAGGT

B AbrplCupPrimer

5' CTTTGGGTTAAGGCTTTCGG

AbrplCdn

5' CAACAGCAGAGCCGGAAACAG

c ATGGCTATTGGTTTAGTCGGTCGCAAATGTGGTATGACTCGCATCTTTACAGATGCTGG TGTTTCTGTACCTGTTACAGTCATCGAAGTCGATCCAAACCGCATTACGCAAACACTTG AAACTGATGGTTATCAAGCTGTTCAAGTAACTACTGGTGAACGTCGCGAGTCTCGCGTA ACTAACGCTCAAAAAGGTCACTTCGCTAAAGCGGGTGTTGCTGCTGGTCGTTTAGTTAA AGAGTTTCGTGTTACTGAAGCTGAGCTTGAAGGCCGTGAAGTTGGCGGTACTATTGGCG TTGATTTGTTCACAGTTGGTCAAATTGTTGACGTAACTGGTCAATCAAAAGGTAAAGGT TTCCAAGGTGGTGTTAAACGTTGGAATTTCCGTACCCAAGATGCTACTCACGGTAACTC TGTTTCTCACCGTGTTTTAGGTTCTACAGGTCAAAAACAAACTCCTGGACGCGTGTTCA AAGGCAAAAAAATGGCTGGTCACTTAGGTGATGAACGCGTAACAGTACAAGGTCTTGAA ATCGTATCTGTTGACACTGAACGTTCAGTTTTGGTTGTTAAGGGTGCAATTCCTGGTGC AACTGGCGGTGACGTTATCGTACGTCCTACCATCAAGGCCTGAGGGGAAATACCGTGAA TTTAA

\begin{tabular}{|c|c|c|c|}
\hline D query & & $\begin{array}{l}\text { MA IGLVGRKCGMTRIFTDAGVSVPVTVIEVDPNRITOIKTLETDGYQAVQVTTGERRE } \\
\text { MAIGLVGRKCGMTRI FTDAGVSVPVTVIEVDPNRITQIKTLETDGYQAVQVTTGERRE }\end{array}$ & 180 \\
\hline Sbjct & 1 & MAIGLVGRKCGMTRIFTDAGVSVPVTVIEVDPNRITQIKTLETDGYQAVQVTTGERRESR & 60 \\
\hline Query & 187 & VTNAOKGHFAKAGVAAGRLVKEFRVTEAELEGREVGGTIGVDLFTVGQIVDVTGQSKGKG & \\
\hline & & VTNAOKGHFAKAGVAAGRLVKEFRVTEAELEGREVGGT I GVDLFTVGOIVDVTGQSKGKG & \\
\hline Sbjct & 61 & VTNAOKGHFAKAGVAAGRLVKEFRVTEAELEGREVGGTI GVDLFTVGQIVDVTGQSKGKG & 120 \\
\hline Query & 367 & FOGGVKRWNFRTODATHGNSVSHRVLGSTGOKOTPGRVFKGKKMAGHLGDERVTVQGLEI & 546 \\
\hline & & FQGGVKRWNFRTQDATHGNSVSHRVLGSTGQ QTPGRVFKGKKMAGHLGDERVTVQGLEI & \\
\hline Sbjet & 121 & FQGGVKRWNFRTQDATHGNSVSHRVLGSTGQNQTPGRVFKGKKMAGHLGDERVTVQGLEI & 180 \\
\hline Query & 547 & VSVDTERSVLVVKGAIPGATGGDVIVRPTIKA & \\
\hline & & VLVVKGAIPGATGGDVIVRPTIKA & \\
\hline Sbjet & 181 & VSVDTERSVLVVKGAIPGATGGDVIVRPTIKA 212 & \\
\hline
\end{tabular}

Figure 6-6. Identification of resistant mechanism of $A$. baumannii to 68 . A) Sequence of A. baumannii 50S ribosomal subunit protein L3 (rplC). B) Primers. C) $r p l C$ Sequence of $\mathbf{6 8}$ Resistant Mutant $\left(\mathbf{6 8}^{\mathrm{R}}\right)$ of $A$. baumannii. D) Blast against Wild type and Mutant $A$. baumannii. (Reprinted with permission. Siricilla, S., Mitachi, K., Yang, J., Eslamimehr, S., Lemieux, M. R., Meibohm, B., Ji, Y., and Kurosu, M. (2017). A new combination of a pleuromutilin derivative and doxycycline for treatment of multidrugresistant Acinetobacter baumannii. J. Med. Chem. American Chemical Society. Article ASAP). 


\section{LIST OF REFERENCES}

1. WHO. (2016) Global Tuberculosis Report 2016. in World Health Organization http://www.who.int/tb/publications/global_report/en/

2. Chien, J. Y., Lai, C. C., Tan, C. K., Chien, S. T., Yu, C. J., and Hsueh, P. R. (2013) Decline in rates of acquired multidrug-resistant tuberculosis after implementation of the directly observed therapy, short course (DOTS) and DOTS-Plus programmes in Taiwan. J Antimicrob Chemother. 68, 1910-1916

3. Ranjbar, S., Boshoff, H. I., Mulder, A., Siddiqi, N., Rubin, E. J., and Goldfeld, A. E. (2009). HIV-1 replication is differentially regulated by distinct clinical strains of Mycobacterium tuberculosis. PLoS One 4, e6116

4. Portero, J. L., and Rubio, M. (2007) New anti-tuberculosis therapies. Expert Opin. Ther. Pat. 17, 617-637

5. Velayati, A. A., Masjedi, M. R., Farnia, P., Tabarsi, P., Ghanavi, J., Ziazarifi, A. H., and Hoffner, S. E. (2009) Emergence of new forms of totally drug-resistant tuberculosis bacilli: super extensively drug-resistant tuberculosis or totally drugresistant strains in iran. Chest 136, 420-425

6. Udwadia, Z. F., Amale, R. A., Ajbani, K. K., and Rodrigues, C. (2012) Totally drug-resistant tuberculosis in India. Clin Infect Dis 54, 579-581

7. Klopper, M., Warren, R. M., Hayes, C., Gey van Pittius, N. C., Streicher, E. M., Muller, B., Sirgel, F. A., Chabula-Nxiweni, M., Hoosain, E., Coetzee, G., David van Helden, P., Victor, T. C., and Trollip, A. P. (2013) Emergence and spread of extensively and totally drug-resistant tuberculosis, South Africa. Emerg Infect Dis 19, $449-455$

8. Connolly, L. E., Edelstein, P. H., and Ramakrishnan, L. (2007) Why is long-term therapy required to cure tuberculosis? PLoS Med 4, e120

9. Nguta, J. M., Appiah-Opong, R., Nyarko, A. K.,Yeboah-Manu, D., Addo, P. G. A. (2015) Current perspectives in drug discovery against tuberculosis from natural products. International J Mycobacteriol 4, 165-183

10. Brennan, P. J. (2003) Structure, function, and biogenesis of the cell wall of Mycobacterium tuberculosis. Tuberculosis (Edinb) 83, 91-97

11. Hett, E. C., and Rubin, E. J. (2008) Bacterial growth and cell division: a mycobacterial perspective. Microbiol Mol Biol Rev 72, 126-156 
12. Warner, D. F., and Mizrahi, V. (2006) Tuberculosis chemotherapy: the influence of bacillary stress and damage response pathways on drug efficacy. Clin Microbiol Rev 19, 558-570

13. Pieters, J. (2008) Mycobacterium tuberculosis and the macrophage: maintaining a balance. Cell Host Microbe 3, 399-407

14. Dutta, N. K., and Karakousis, P. C. (2014) Latent tuberculosis infection: myths, models, and molecular mechanisms. Microbiol Mol Biol Rev 78, 343-371

15. FDA. Sirturo (bedaquiline) product insert. Silver Spring, MD: Food and Drug Administration http://www.accessdata.fda.gov/drugsatfda_docs/label/2012/204384s000lbl.pdf

16. Agency, E. M. Deltyba: EPAR-Summary for the public. http://www.ema.europa.eu/docs/en_GB/document library/EPAR_Summary for the public/human/002552/WC500166235.pdf

17. NIH. Division of AIDS Anti-HIV/OI/TB Therapeutics Database. https://chemdb.niaid.nih.gov/DrugDevelopmentTB.aspx

18. StopTBPartnership. http://www.newtbdrugs.org/pipeline/trials/evaluation-sq109high-dose-rifampicin-and-moxifloxacin-adults-smear-positive-0

19. Singh, R., Manjunatha, U., Boshoff, H. I., Ha, Y. H., Niyomrattanakit, P., Ledwidge, R., Dowd, C. S., Lee, I. Y., Kim, P., Zhang, L., Kang, S., Keller, T. H., Jiricek, J., and Barry, C. E., 3rd. (2008) PA-824 kills nonreplicating Mycobacterium tuberculosis by intracellular NO release. Science 322, 1392-1395

20. Manjunatha, U., Boshoff, H. I., and Barry, C. E. (2009) The mechanism of action of PA-824: Novel insights from transcriptional profiling. Commun Integr Biol 2, $215-218$

21. Stover, C. K., Warrener, P., VanDevanter, D. R., Sherman, D. R., Arain, T. M., Langhorne, M. H., Anderson, S. W., Towell, J. A., Yuan, Y., McMurray, D. N., Kreiswirth, B. N., Barry, C. E., and Baker, W. R. (2000) A small-molecule nitroimidazopyran drug candidate for the treatment of tuberculosis. Nature 405, 962-966

22. Lessem, E. The tuberculosis treatment pipeline: Activity, but no answers. http://pipelinereport.org/2016/tb-treatment

23. TB Alliance. PaMZ. New York: TB Alliance. http://www.tballiance.org/portfolio/regimen/pamz 
24. ClinicalTrials.gov. https://clinicaltrials.gov/ct2/show/NCT00567840?term=pa$\underline{824 \& \mathrm{rank}=6}$

25. Infectex, M. B. V. F., Sequella Incorporated (Press Release). . (2012 December 19.) Maxwell Biotech Venture Fund's portfolio company, Infectex, enrolls first multi-drug resistant tuberculosis (MDR-TB) patients in pivotal clinical trial of SQ109, licensed from Sequella

26. Phipps, A. (2016; April 4-7) Sequella, Inc. Oral presentation at: TB drug codevelopment roundtable: brief industry updates

27. Boeree, M., and Hoelscher, M. (2015; February 23-26) High-dose rifampin, SQ109 and moxifloxacin for treating TB: the PanACEA MAMS-TB trial. in Paper presented at: 22nd Conference on Retroviruses and Opportunistic Infections, Seattle, WA

28. Astarie-Dequeker, C., N'Diaye, E. N., Le Cabec, V., Rittig, M. G., Prandi, J., and Maridonneau-Parini, I. (1999) The mannose receptor mediates uptake of pathogenic and nonpathogenic mycobacteria and bypasses bactericidal responses in human macrophages. Infect Immun 67, 469-477

29. Podinovskaia, M., Lee, W., Caldwell, S., and Russell, D. G. (2013) Infection of macrophages with Mycobacterium tuberculosis induces global modifications to phagosomal function. Cell Microbiol 15, 843-859

30. Rustad, T. R., Sherrid, A. M., Minch, K. J., and Sherman, D. R. (2009) Hypoxia: a window into Mycobacterium tuberculosis latency. Cell Microbiol 11, 11511159

31. Rodriguez, J. G., Hernandez, A. C., Helguera-Repetto, C., Aguilar Ayala, D., Guadarrama-Medina, R., Anzola, J. M., Bustos, J. R., Zambrano, M. M., Gonzalez, Y. M. J., Garcia, M. J., and Del Portillo, P. (2014) Global adaptation to a lipid environment triggers the dormancy-related phenotype of Mycobacterium tuberculosis. MBio 5, e01125-01114

32. Yoshida, A., Inagawa, H., Kohchi, C., Nishizawa, T., and Soma, G. (2009) The role of toll-like receptor 2 in survival strategies of Mycobacterium tuberculosis in macrophage phagosomes. Anticancer Res 29, 907-910

33. Achkar, J. M., and Jenny-Avital, E. R. (2011) Incipient and subclinical tuberculosis: defining early disease states in the context of host immune response. J Infect Dis 204 Suppl 4, S1179-1186

34. Silva Miranda, M., Breiman, A., Allain, S., Deknuydt, F., and Altare, F. (2012) The tuberculous granuloma: an unsuccessful host defence mechanism providing a safety shelter for the bacteria? Clin Dev Immunol 2012, 139127 
35. Kurosu, M., and William, D. (2016) New advances in tuberculosis drug discovery. Future Science 20, 49-70

36. Ehmann, D. E., and Lahiri, S. D. (2014) Novel compounds targeting bacterial DNA topoisomerase/DNA gyrase. Curr Opin Pharmacol 18, 76-83

37. Fernandez, P., Saint-Joanis, B., Barilone, N., Jackson, M., Gicquel, B., Cole, S. T., and Alzari, P. M. (2006) The Ser/Thr protein kinase PknB is essential for sustaining mycobacterial growth. J Bacteriol 188, 7778-7784

38. Converse, P. J., Karakousis, P. C., Klinkenberg, L. G., Kesavan, A. K., Ly, L. H., Allen, S. S., Grosset, J. H., Jain, S. K., Lamichhane, G., Manabe, Y. C., McMurray, D. N., Nuermberger, E. L., and Bishai, W. R. (2009) Role of the dosR-dosS two-component regulatory system in Mycobacterium tuberculosis virulence in three animal models. Infect Immun 77, 1230-1237

39. Sivaramakrishnan, S., and de Montellano, P. R. (2013) The DosS-DosT/DosR Mycobacterial Sensor System. Biosensors (Basel) 3, 259-282

40. McKinney, J. D., Honer zu Bentrup, K., Munoz-Elias, E. J., Miczak, A., Chen, B., Chan, W. T., Swenson, D., Sacchettini, J. C., Jacobs, W. R., Jr., and Russell, D. G. (2000) Persistence of Mycobacterium tuberculosis in macrophages and mice requires the glyoxylate shunt enzyme isocitrate lyase. Nature 406, 735-738

41. Mdluli, K., and Spigelman, M. (2006) Novel targets for tuberculosis drug discovery. Curr Opin Pharmacol 6, 459-467

42. Sherman, M. M., Petersen, L. A., and Poulter, C. D. (1989) Isolation and characterization of isoprene mutants of Escherichia coli. J Bacteriol 171, 36193628

43. Unden, G., and Bongaerts, J. (1997) Alternative respiratory pathways of Escherichia coli: energetics and transcriptional regulation in response to electron acceptors. Biochim Biophys Acta 1320, 217-234

44. Bishop, D. H., Pandya, K. P., and King, H. K. (1962) Ubiquinone and vitamin K in bacteria. Biochem $J$ 83, 606-614

45. Dhiman, R. K., Mahapatra, S., Slayden, R. A., Boyne, M. E., Lenaerts, A., Hinshaw, J. C., Angala, S. K., Chatterjee, D., Biswas, K., Narayanasamy, P., Kurosu, M., and Crick, D. C. (2009) Menaquinone synthesis is critical for maintaining mycobacterial viability during exponential growth and recovery from non-replicating persistence. Mol Microbiol 72, 85-97 
46. Rao, S. P. S., Alonso, S., Rand, L., Dick, T., and Pethe, K. (2008) The protonmotive force is required for maintaining ATP homeostasis and viability of hypoxic, nonreplicating Mycobacterium tuberculosis PNAS 105, 11945-11950

47. Cho, S. H., Warit, S., Wan, B., Hwang, C. H., Pauli, G. F., and Franzblau, S. G. (2007) Low-oxygen-recovery assay for high-throughput screening of compounds against nonreplicating Mycobacterium tuberculosis. Antimicrob Agents Chemother 51, 1380-1385

48. Zhang, M., Sala, C., Dhar, N., Vocat, A., Sambandamurthy, V. K., Sharma, S., Marriner, G., Balasubramanian, V., and Cole, S. T. (2014) In vitro and in vivo activities of three oxazolidinones against nonreplicating Mycobacterium tuberculosis. Antimicrob Agents Chemother 58, 3217-3223

49. Vera-Cabrera, L., Gonzalez, E., Rendon, A., Ocampo-Candiani, J., Welsh, O., Velazquez-Moreno, V. M., Choi, S. H., and Molina-Torres, C. (2006) In vitro activities of DA-7157 and DA-7218 against Mycobacterium tuberculosis and Nocardia brasiliensis. Antimicrob Agents Chemother 50, 3170-3172

50. Lee, R. E., Hurdle, J. G., Liu, J., Bruhn, D. F., Matt, T., Scherman, M. S., Vaddady, P. K., Zheng, Z., Qi, J., Akbergenov, R., Das, S., Madhura, D. B., Rathi, C., Trivedi, A., Villellas, C., Lee, R. B., Rakesh, Waidyarachchi, S. L., Sun, D., McNeil, M. R., Ainsa, J. A., Boshoff, H. I., Gonzalez-Juarrero, M., Meibohm, B., Bottger, E. C., and Lenaerts, A. J. (2014) Spectinamides: a new class of semisynthetic antituberculosis agents that overcome native drug efflux. Nat Med 20, 152-158

51. Huang, T. S., Liu, Y. C., Sy, C. L., Chen, Y. S., Tu, H. Z., and Chen, B. C. (2008) In vitro activities of linezolid against clinical isolates of Mycobacterium tuberculosis complex isolated in Taiwan over 10 years. Antimicrob Agents Chemother 52, 2226-2227

52. Lopez-Gavin, A., Tudo, G., Vergara, A., Hurtado, J. C., and Gonzalez-Martin, J. (2015) In vitro activity against Mycobacterium tuberculosis of levofloxacin, moxifloxacin and UB-8902 in combination with clofazimine and pretomanid. Int $J$ Antimicrob Agents 46, 582-585

53. Werngren, J., Wijkander, M., Perskvist, N., Balasubramanian, V., Sambandamurthy, V. K., Rodrigues, C., and Hoffner, S. (2014) In vitro activity of AZD5847 against geographically diverse clinical isolates of Mycobacterium tuberculosis. Antimicrob Agents Chemother 58, 4222-4223

54. Ji, B., Lounis, N., Maslo, C., Truffot-Pernot, C., Bonnafous, P., and Grosset, J. (1998) In vitro and in vivo activities of moxifloxacin and clinafloxacin against Mycobacterium tuberculosis. Antimicrob Agents Chemother 42, 2066-2069 
55. Alvirez-Freites, E. J., Carter, J. L., and Cynamon, M. H. (2002) In vitro and in vivo activities of gatifloxacin against Mycobacterium tuberculosis. Antimicrob. Agents Chemother. 46, 1022-1025

56. Demet, U. S., Barkin, B., Ece, G". U., Nihan, A., Tanıl, K. O., Berrak, C. A. G. L., I,sil, A. K., and Dilek, D. I. E. (2010) Mannich base derivatives of 3-hydroxy6-methyl-4H-pyran-4-one with antimicrobial activity. Turk. J. Chem. 34, 447-456

57. P, S. H., Solapure, S., Mukherjee, K., Nandi, V., Waterson, D., Shandil, R., Balganesh, M., Sambandamurthy, V. K., Raichurkar, A. K., Deshpande, A., Ghosh, A., Awasthy, D., Shanbhag, G., Sheikh, G., McMiken, H., Puttur, J., Reddy, J., Werngren, J., Read, J., Kumar, M., R, M., Chinnapattu, M., Madhavapeddi, P., Manjrekar, P., Basu, R., Gaonkar, S., Sharma, S., Hoffner, S., Humnabadkar, V., Subbulakshmi, V., and Panduga, V. (2014) Optimization of pyrrolamides as mycobacterial GyrB ATPase inhibitors: structure-activity relationship and in vivo efficacy in a mouse model of tuberculosis. Antimicrob Agents Chemother 58, 61-70

58. Chopra, S., Matsuyama, K., Tran, T., Malerich, J. P., Wan, B., Franzblau, S. G., Lun, S., Guo, H., Maiga, M. C., Bishai, W. R., and Madrid, P. B. (2012) Evaluation of gyrase B as a drug target in Mycobacterium tuberculosis. $J$ Antimicrob Chemother 67, 415-421

59. Kale, R. R., Kale, M. G., Waterson, D., Raichurkar, A., Hameed, S. P., Manjunatha, M. R., Kishore Reddy, B. K., Malolanarasimhan, K., Shinde, V., Koushik, K., Jena, L. K., Menasinakai, S., Humnabadkar, V., Madhavapeddi, P., Basavarajappa, H., Sharma, S., Nandishaiah, R., Mahesh Kumar, K. N., Ganguly, S., Ahuja, V., Gaonkar, S., Naveen Kumar, C. N., Ogg, D., Boriack-Sjodin, P. A., Sambandamurthy, V. K., de Sousa, S. M., and Ghorpade, S. R. (2014) Thiazolopyridone ureas as DNA gyrase B inhibitors: optimization of antitubercular activity and efficacy. Bioorg Med Chem Lett 24, 870-879

60. Gupta, R. K., Thakur, T. S., Desiraju, G. R., and Tyagi, J. S. (2009) Structurebased design of DevR inhibitor active against nonreplicating Mycobacterium tuberculosis. J Med Chem 52, 6324-6334

61. Kurosu, M., and Begari, E. (2010) Bacterial Protein Kinase Inhibitors. Drug Develop. Research 71, 168-187

62. Walburger, A., Koul, A., Ferrari, G., Nguyen, L., Prescianotto-Baschong, C., Huygen, K., Klebl, B., Thompson, C., Bacher, G., and Pieters, J. (2004) Protein kinase $\mathrm{G}$ from pathogenic mycobacteria promotes survival within macrophages. Science 304, 1800-1804

63. Hegymegi-Barakonyi, B., Szekely, R., Varga, Z., Kiss, R., Borbely, G., Nemeth, G., Banhegyi, P., Pato, J., Greff, Z., Horvath, Z., Meszaros, G., Marosfalvi, J., 
Eros, D., Szantai-Kis, C., Breza, N., Garavaglia, S., Perozzi, S., Rizzi, M., Hafenbradl, D., Ko, M., Av-Gay, Y., Klebl, B. M., Orfi, L., and Keri, G. (2008) Signalling inhibitors against Mycobacterium tuberculosis--early days of a new therapeutic concept in tuberculosis. Curr Med Chem 15, 2760-2770

64. Szekely, R., Waczek, F., Szabadkai, I., Nemeth, G., Hegymegi-Barakonyi, B., Eros, D., Szokol, B., Pato, J., Hafenbradl, D., Satchell, J., Saint-Joanis, B., Cole, S. T., Orfi, L., Klebl, B. M., and Keri, G. (2008) A novel drug discovery concept for tuberculosis: inhibition of bacterial and host cell signalling. Immunol Lett 116, 225-231

65. Lougheed, K. E., Osborne, S. A., Saxty, B., Whalley, D., Chapman, T., Bouloc, N., Chugh, J., Nott, T. J., Patel, D., Spivey, V. L., Kettleborough, C. A., Bryans, J. S., Taylor, D. L., Smerdon, S. J., and Buxton, R. S. (2011) Effective inhibitors of the essential kinase PknB and their potential as anti-mycobacterial agents. Tuberculosis (Edinb) 91, 277-286

66. Ji, L., Long, Q., Yang, D., and Xie, J. (2011) Identification of mannich base as a novel inhibitor of Mycobacterium tuberculosis isocitrate by high-throughput screening. Int J Biol Sci 7, 376-382

67. Sriram, D., Yogeeswari, P., Methuku, S., Vyas, D. R., Senthilkumar, P., Alvala, M., and Jeankumar, V. U. (2011) Synthesis of various 3-nitropropionamides as Mycobacterium tuberculosis isocitrate lyase inhibitor. Bioorg Med Chem Lett 21, 5149-5154

68. Porntep, C., Suthep, W., Nongluksna, S., Nattaya, N., Duangnate, I., and Prasat, K. (2005) 3-Nitropropionic acid (3-NPA), a potent antimycobacterial agent from endophytic fungi: Is 3-NPA in some plants produced by endophytes? J. Nat. Prod. 68, 1103-1105

69. Krieger, I. V., Freundlich, J. S., Gawandi, V. B., Roberts, J. P., Gawandi, V. B., Sun, Q., Owen, J. L., Fraile, M. T., Huss, S. I., Lavandera, J. L., Ioerger, T. R., and Sacchettini, J. C. (2012) Structure-guided discovery of phenyl-diketo acids as potent inhibitors of M. tuberculosis malate synthase. Chem Biol 19, 1556-1567

70. Kratky, M., Vinsova, J., Novotna, E., Mandikova, J., Wsol, V., Trejtnar, F., Ulmann, V., Stolarikova, J., Fernandes, S., Bhat, S., and Liu, J. O. (2012)

Salicylanilide derivatives block Mycobacterium tuberculosis through inhibition of isocitrate lyase and methionine aminopeptidase. Tuberculosis (Edinb) 92, 434-439

71. Liu, Y., Zhou, S., Deng, Q., Li, X., Meng, J., Guan, Y., Li, C., and Xiao, C. (2016) Identification of a novel inhibitor of isocitrate lyase as a potent antitubercular agent against both active and non-replicating Mycobacterium tuberculosis. Tuberculosis (Edinb) 97, 38-46 
72. Wayne, L. G., and Sramek, H. A. (1994) Metronidazole is bactericidal to dormant cells of Mycobacterium tuberculosis. Antimicrob Agents Chemother 38, 20542058

73. Iona, E., Giannoni, F., Pardini, M., Brunori, L., Orefici, G., and Fattorini, L. (2007) Metronidazole plus rifampin sterilizes long-term dormant Mycobacterium tuberculosis. Antimicrob Agents Chemother 51, 1537-1540

74. Lenaerts, A. J., Gruppo, V., Marietta, K. S., Johnson, C. M., Driscoll, D. K., Tompkins, N. M., Rose, J. D., Reynolds, R. C., and Orme, I. M. (2005) Preclinical testing of the nitroimidazopyran PA-824 for activity against Mycobacterium tuberculosis in a series of in vitro and in vivo models. Antimicrob Agents Chemother 49, 2294-2301

75. Stinson, K., Kurepina, N., Venter, A., Fujiwara, M., Kawasaki, M., Timm, J., Shashkina, E., Kreiswirth, B. N., Liu, Y., Matsumoto, M., and Geiter, L. (2016) MIC of delamanid (OPC-67683) against Mycobacterium tuberculosis clinical isolates and a proposed critical concentration. Antimicrob Agents Chemother 60, 3316-3322

76. Upton, A. M., Cho, S., Yang, T. J., Kim, Y., Wang, Y., Lu, Y., Wang, B., Xu, J., Mdluli, K., Ma, Z., and Franzblau, S. G. (2015) In vitro and in vivo activities of the nitroimidazole TBA-354 against Mycobacterium tuberculosis. Antimicrob Agents Chemother 59, 136-144

77. Weinstein, E. A., Yano, T., Li, L. S., Avarbock, D., Avarbock, A., Helm, D., McColm, A. A., Duncan, K., Lonsdale, J. T., and Rubin, H. (2005) Inhibitors of type II NADH:menaquinone oxidoreductase represent a class of antitubercular drugs. Proc Natl Acad Sci U S A 102, 4548-4553

78. Crowle, A. J., Douvas, G. S., and May, M. H. (1992) Chlorpromazine: a drug potentially useful for treating mycobacterial infections. Chemotherapy $\mathbf{3 8}, 410-$ 419

79. Advani, M. J., Siddiqui, I., Sharma, P., and Reddy, H. (2012) Activity of trifluoperazine against replicating, non-replicating and drug resistant $M$. tuberculosis. PLoS One 7, e44245

80. Amaral, L., Kristiansen, J. E., Viveiros, M., and Atouguia, J. (2001) Activity of phenothiazines against antibiotic-resistant Mycobacterium tuberculosis: a review supporting further studies that may elucidate the potential use of thioridazine as anti-tuberculosis therapy. J Antimicrob Chemother 47, 505-511

81. Sohaskey, C. (2011) Latent tuberculosis: is there a role for thioridazine? Recent Pat Antiinfect Drug Discov 6, 139-146 
82. Lakshminarayana, S. B., Huat, T. B., Ho, P. C., Manjunatha, U. H., Dartois, V., Dick, T., and Rao, S. P. (2015) Comprehensive physicochemical, pharmacokinetic and activity profiling of anti-TB agents. J Antimicrob Chemother 70, 857-867

83. Debnath, J., Siricilla, S., Wan, B., Crick, D. C., Lenaerts, A. J., Franzblau, S. G., and Kurosu, M. (2012) Discovery of selective menaquinone biosynthesis inhibitors against Mycobacterium tuberculosis. J Med Chem 55, 3739-3755

84. Choi, S. R., Larson, M. A., Hinrichs, S. H., Bartling, A. M., Frandsen, J., and Narayanasamy, P. (2016) Discovery of bicyclic inhibitors against menaquinone biosynthesis. Future Med Chem 8, 11-16

85. Li, X., Liu, N., Zhang, H., Knudson, S. E., Li, H. J., Lai, C. T., Simmerling, C., Slayden, R. A., and Tonge, P. J. (2011) CoA adducts of 4-oxo-4-phenylbut-2enoates: Inhibitors of MenB from the M. tuberculosis menaquinone biosynthesis pathway. ACS Med Chem Lett 2, 818-823

86. Matarlo, J. S., Lu, Y., Daryaee, F., Daryaee, T., Ruzsicska, B., Walker, S.G., and Tonge, P.J. (2016) A methyl 4 $\square$ oxo-4-phenylbut-2-enoate with in vivo activity against MRSA that inhibits MenB in the bacterial menaquinone biosynthesis pathway $J$ Infect Dis 2, 329-340

87. Lu, X., Zhou, R., Sharma, I., Li, X., Kumar, G., Swaminathan, S., Tonge, P.J., and Tan, D.S. (2012) Stable analogues of OSB-AMP: potent inhibitors of MenE, the o-succinylbenzoate-CoA synthetase from bacterial menaquinone biosynthesis. Chem Bio Chem 13, 129-136

88. Lu, X., Zhang, H., Tonge, P. J., and Tan, D. S. (2008) Mechanism-based inhibitors of MenE, an acyl-CoA synthetase involved in bacterial menaquinone biosynthesis. Bioorg Med Chem Lett 18, 5963-5966

89. Koul, A., Vranckx, L., Dendouga, N., Balemans, W., Van den Wyngaert, I., Vergauwen, K., Gohlmann, H. W., Willebrords, R., Poncelet, A., Guillemont, J., Bald, D., and Andries, K. (2008) Diarylquinolines are bactericidal for dormant mycobacteria as a result of disturbed ATP homeostasis. J Biol Chem 283, 2527325280

90. Ishizaki, Y., Hayashi, C., Inoue, K., Igarashi, M., Takahashi, Y., Pujari, V., Crick, D. C., Brennan, P. J., and Nomoto, A. (2013) Inhibition of the first step in synthesis of the mycobacterial cell wall core, catalyzed by the GlcNAc-1phosphate transferase WecA, by the novel caprazamycin derivative CPZEN-45. $J$ Biol Chem 288, 30309-30319

91. Siricilla, S., Mitachi, K., Wan, B., Franzblau, S. G., and Kurosu, M. (2015) Discovery of a capuramycin analog that kills nonreplicating Mycobacterium 
tuberculosis and its synergistic effects with translocase I inhibitors. $J$ Antibiot (Tokyo) 68, 271-278

92. Hugonnet, J. E., Tremblay, L. W., Boshoff, H. I., Barry, C. E., 3rd, and Blanchard, J. S. (2009) Meropenem-clavulanate is effective against extensively drug-resistant Mycobacterium tuberculosis. Science 323, 1215-1218

93. Gold, B., Smith, R., Nguyen, Q., Roberts, J., Ling, Y., Lopez Quezada, L., Somersan, S., Warrier, T., Little, D., Pingle, M., Zhang, D., Ballinger, E., Zimmerman, M., Dartois, V., Hanson, P., Mitscher, L. A., Porubsky, P., Rogers, S., Schoenen, F. J., Nathan, C., and Aube, J. (2016) Novel cephalosporins selectively active on nonreplicating Mycobacterium tuberculosis. J Med Chem 59, 6027-6044

94. Murphy, D. J., and Brown, J. R. (2007) Identification of gene targets against dormant phase Mycobacterium tuberculosis infections. BMC Infect Dis 7, 84

95. Hirokawa, T., Boon-Chieng, S., and Mitaku, S. (1998) SOSUI: classification and secondary structure prediction system for membrane proteins. Bioinformatics 14, $378-379$

96. Kurosu, M., and Begari, E. (2010) Vitamin $\mathrm{K}_{2}$ in electron transport system: are enzymes involved in vitamin $\mathrm{K}_{2}$ biosynthesis promising drug targets? Molecules 15, 1531-1553

97. Kurosu, M., Narayanasamy, P., Biswas, K., Dhiman, R., and Crick, D. C. (2007) Discovery of 1,4-dihydroxy-2-naphthoate [corrected] prenyltransferase inhibitors: new drug leads for multidrug-resistant gram-positive pathogens. J Med Chem 50, 3973-3975

98. Kurosu, M., and Crick, D. C. (2009) MenA is a promising drug target for developing novel lead molecules to combat Mycobacterium tuberculosis. Med Chem 5, 197-207

99. Xu, H., Graham, M., Karelis, J., Walker, S. G., Peter, J., and Tonge, P. J. (2009) Mechanistic studies of MenD, 2-succinyl-5-enoylpyruvyl-6-hydroxy-3cyclohexene-1-carboxylic acid synthase from Staphylococcus aureus. in In 237th ACS National Meeting, Salt Lake City, UT, USA

100. Barry, C. E., and Blanchard, J. S. (2010) The chemical biology of new drugs in the development for tuberculosis. Curr Opin Chem Biol 14, 456-466

101. Yue, J., Yi, X., Wenli, Z., and Yufang, M. (2010) Mycobacterium tuberculosis Rv1302 and Mycobacterium smegmatis MSMEG_4947 have WecA function and MSMEG_4947 is required for the growth of M. smegmatis. FEMS Microbiol Lett 310, $54-61$ 
102. Bacon, J., Alderwick, L. J., Allnutt, J. A., Gabasova, E., Watson, R., Hatch, K. A., Clark, S. O., Jeeves, R. E., Marriott, A., Rayner, E., Tolley, H., Pearson, G., Hall, G., Besra, G. S., Wernisch, L., Williams, A., and Marsh, P. D. (2014) Nonreplicating Mycobacterium tuberculosis elicits a reduced infectivity profile with corresponding modifications to the cell wall and extracellular matrix. PLoS One 9, e87329

103. Mitachi, K., Siricilla, S., Yang, D., Kong, Y., Skorupinska-Tudek, K., Swiezewska, E., Franzblau, S. G., and Kurosu, M. (2016) Fluorescence-based assay for polyprenyl phosphate-GlcNAc-1-phosphate transferase (WecA) and identification of novel antimycobacterial WecA inhibitors. Anal Biochem 512, 7890

104. Berg, S., Kaur, D., Jackson, M., and Brennan, P. J. (2007) The glycosyltransferases of Mycobacterium tuberculosis - roles in the synthesis of arabinogalactan, lipoarabinomannan, and other glycoconjugates. Glycobiology 17, $35-56 \mathrm{R}$

105. Price, N. P., and Momany, F. A. (2005) Modeling bacterial UDP-HexNAc: polyprenol-P HexNAc-1-P transferases. Glycobiology 15, 29R-42R

106. Lukose, V., Luo, L., Kozakov, D., Vajda, S., Allen, K. N., and Imperiali, B. (2015) Conservation and covariance in small bacterial phosphoglycosyltransferases identify the functional catalytic core. Biochemistry 54, 7326-7334

107. Schaffer, C., Wugeditsch, T., Messner, P., and Whitfield, C. (2002) Functional expression of enterobacterial O-polysaccharide biosynthesis enzymes in Bacillus subtilis. Appl Environ Microbiol 68, 4722-4730

108. Timothy, D. H., Lloyd, A.J., and Roper, D.I. (2006) Phospho-MurNAcpentapeptide translocase (MraY) as a target for antibacterial agents and antibacterial proteins. Infect. Disord. Drug Targets. 6, 85-106

109. Brandish, P. E., Kimura, K. I., Inukai, M., Southgate, R., Lonsdale, J. T., and Bugg, T. D. (1996) Modes of action of tunicamycin, liposidomycin B, and mureidomycin A: inhibition of phospho-N-acetylmuramyl-pentapeptide translocase from Escherichia coli. Antimicrob Agents Chemother 40, 1640-1644

110. Mitachi, K., Aleiwi, B., Schneider, C., Siricilla, S., and Kurosu, M. (2016) Stereocontrolled total synthesis of muraymycin D1 having a dual modeof action against Mycobacterium tuberculosis. J Amer Chem Soc 138, 12975-12980

111. Vicens, Q., and Westhof, E. (2002) Crystal structure of a complex between the aminoglycoside tobramycin and an oligonucleotide containing the ribosomal decoding a site. Chem Biol 9, 747-755 
112. Ippolito, J. A., Kanyo, Z. F., Wang, D., Franceschi, F. J., Moore, P. B., Steitz, T. A., and Duffy, E. M. (2008) Crystal structure of the oxazolidinone antibiotic linezolid bound to the 50S ribosomal subunit. J Med Chem 51, 3353-3356

113. Xie, J., Talaska, A. E., and Schacht, J. (2011) New developments in aminoglycoside therapy and ototoxicity. Hear Res 281, 28-37

114. Melchionda, V., Wyatt, H., Capocci, S., Garcia Medina, R., Solamalai, A., Katiri, S., Hopkins, S., Cropley, I., and Lipman, M. (2013) Amikacin treatment for multidrug resistant tuberculosis: how much monitoring is required? Eur Respir $J$ 42, $1148-1150$

115. Maartens, G., and Benson, C. A. (2015) Linezolid for treating tuberculosis: A delicate balancing act. EBioMedicine 2, 1568-1569

116. Zhang, X., Falagas, M. E., Vardakas, K. Z., Wang, R., Qin, R., Wang, J., and Liu, Y. (2015) Systematic review and meta-analysis of the efficacy and safety of therapy with linezolid containing regimens in the treatment of multidrug-resistant and extensively drug-resistant tuberculosis. J Thorac Dis 7, 603-615

117. Poulsen, S. M., Karlsson, M., Johansson, L. B., and Vester, B. (2001) The pleuromutilin drugs tiamulin and valnemulin bind to the RNA at the peptidyl transferase centre on the ribosome. Mol. Microbiol. 41, 1091-1099

118. Schlunzen, F., Pyetan, E., Fucini, P., Yonath, A., and Harms, J. M. (2004) Inhibition of peptide bond formation by pleuromutilins: the structure of the $50 \mathrm{~S}$ ribosomal subunit from Deinococcus radiodurans in complex with tiamulin. Mol Microbiol 54, 1287-1294

119. Miller, K., Dunsmore, C. J., Fishwick, C. W., and Chopra, I. (2008) Linezolid and tiamulin cross-resistance in Staphylococcus aureus mediated by point mutations in the peptidyl transferase center. Antimicrob Agents Chemother 52, 1737-1742

120. Kitagawa, W., and Tamura, T. (2008) A quinoline antibiotic from Rhodococcus erythropolis JCM 6824. J Antibiot (Tokyo) 61, 680-682

121. Mitchell, P. (1961) Coupling of phosphorylation to electron and hydrogen transfer by a chemi-osmotic type of mechanism. Nature 191, 144-148

122. Mitchell, P. (1966) Chemiosmotic coupling in oxidative and photosynthetic phosphorylation. Biol Rev Camb Philos Soc 41, 445-502

123. Mitchell, P. (1972) Chemiosmotic coupling in energy transduction: a logical development of biochemical knowledge. J Bioenerg 3, 5-24 
124. Haddock, B. A., and Jones, C. W. (1977) Bacterial respiration. Bacteriol Rev. 41, 47-99

125. Anraku, Y. (1988) Bacterial electron transport chains. Annu Rev Biochem 57, $101-132$

126. Andries, K., Verhasselt, P., Guillemont, J., Gohlmann, H. W., Neefs, J. M., Winkler, H., Van Gestel, J., Timmerman, P., Zhu, M., Lee, E., Williams, P., de Chaffoy, D., Huitric, E., Hoffner, S., Cambau, E., Truffot-Pernot, C., Lounis, N., and Jarlier, V. (2005) A diarylquinoline drug active on the ATP synthase of Mycobacterium tuberculosis. Science 307, 223-227

127. Zhang, H., and Tonge, P. J. (2007) The mechanism of the reactions catalyzed by 1,4-dihydroxynaphthoyl-CoA synthase (MenB) and 2-ketocyclohexanecarboxylCoA hydrolase (BadI). Abstract in 234th ACS National Meeting; Boston, MA, United States. Abstract in 234th 19-23

128. Li, X., Zhang, H., and Tonge, P. J. (2008) Inhibition of 1,4-dihydroxynaphthoylCoA synthase (MenB), an enzyme drug target bacterial menaquinone biosynthesis pathway. Abstract in 236th ACS National Meeting; Philadelphia, PA, United States 236, 17-21

129. Xu, H., Graham, M., Karelis, J., Walker, S. G., Peter, J., and Tonge, P. J. (2009) Mechanistic studies of MenD, 2-succinyl-5-enoylpyruvyl-6-hydroxy-3cyclohexene-1-carboxylic acid synthase from Staphylococcus aureus. Abstract in 237th ACS National Meeting; Salt Lake City, UT. 237, 22-26

130. Barry, P. J., and O'Connor, T. M. (2007) Novel agents in the management of Mycobacterium tuberculosis disease. Curr Med Chem 14, 2000-2008

131. Altschul, S. F., Gish, W., Miller, W., Myers, E. W., and Lipman, D. J. (1990) Basic local alignment search tool. J Mol Biol 215, 403-410

132. Boshoff, H. I., Myers, T. G., Copp, B. R., McNeil, M. R., Wilson, M. A., and Barry, C. E. (2004) The transcriptional responses of Mycobacterium tuberculosis to inhibitors of metabolism: novel insights into drug mechanisms of action. $\mathrm{J} \mathrm{Biol}$ Chem. 279, 40174-40184

133. Mathew, R., Kruthiventi, A. K., Prasad, J. V., Kumar, S. P., Srinu, G., and Chatterji, D. (2010) Inhibition of mycobacterial growth by plumbagin derivatives. Chem Biol Drug Des 76, 34-42

134. Collins, L., and Franzblau, S. G. (1997) Microplate alamar blue assay versus BACTEC 460 system for high-throughput screening of compounds against Mycobacterium tuberculosis and Mycobacterium avium. Antimicrob Agents Chemother 41, 1004-1009 
135. Wayne, L. G., and Sramek, H. A. (1979) Antigenic differences between extracts of actively replicating and synchronized resting cells of Mycobacterium tuberculosis. Infect Immun. 24, 363-370

136. Wayne, L. G., and Hayes, L. G. (1996) An in vitro model for sequential study of shiftdown of Mycobacterium tuberculosis through two stages of nonreplicating persistence. Infect Immun. 64, 2062-2069

137. Loebel, R. O., Shorr, E., and Richardson, H. B. (1933) The influence of adverse conditions upon the respiratory metabolism and growth of human tubercle bacilli. J Bacteriol 26, 167-200

138. Honaker, R. W., Leistikow, R. L., Bartek, I. L., and Voskuil, M. I. (2009) Unique roles of DosT and DosS in DosR regulon induction and Mycobacterium tuberculosis dormancy. Infect Immun 77, 3258-3263

139. Grzegorzewicz, A. E., Ma, Y., Jones, V., Crick, D., Liav, A., and McNeil, M. R. (2008) Development of a microtitre plate-based assay for lipid-linked glycosyltransferase products using the mycobacterial cell wall rhamnosyltransferase WbbL. Microbiology 154, 3724-3730

140. Dhar, N., Dubee, V., Ballell, L., Cuinet, G., Hugonnet, J. E., Signorino-Gelo, F., Barros, D., Arthur, M., and McKinney, J. D. (2015) Rapid cytolysis of Mycobacterium tuberculosis by faropenem, an orally bioavailable beta-lactam antibiotic. Antimicrob Agents Chemother 59, 1308-1319

141. Siricilla, S., Mitachi, K., Skorupinska-Tudek, K., Swiezewska, E., and Kurosu, M. (2014) Biosynthesis of a water-soluble lipid I analogue and a convenient assay for translocase I. Anal Biochem 461, 36-45

142. Bogatcheva, E., Dubuisson, T., Protopopova, M., Einck, L., Nacy, C. A., and Reddy, V. M. (2011) Chemical modification of capuramycins to enhance antibacterial activity. $J$ Antimicrob Chemother 66, 578-587

143. Koga, T., Fukuoka, T., Doi, N., Harasaki, T., Inoue, H., Hotoda, H., Kakuta, M., Muramatsu, Y., Yamamura, N., Hoshi, M., and Hirota, T. (2004) Activity of capuramycin analogues against Mycobacterium tuberculosis, Mycobacterium avium and Mycobacterium intracellulare in vitro and in vivo. J Antimicrob Chemother 54, 755-760

144. Mitachi, K., Aleiwi, B. A., Schneider, C. M., Siricilla, S., and Kurosu, M. (2016) Stereocontrolled total synthesis of muraymycin D1 having a dual mode of action against Mycobacterium tuberculosis. J Am Chem Soc 138, 12975-12980 
145. Kurosu, M., Mahapatra, S., Narayanasamy, P., and Crick, D. C. (2007)

Chemoenzymatic synthesis of park's nucleotide: toward the development of highthroughput screening for MraY inhibitors. Tetrahedron Lett. 48, 799-803

146. Bugg, T. D., Lloyd, A. J., and Roper, D. I. (2006) Phospho-MurNAc-pentapeptide translocase (MraY) as a target for antibacterial agents and antibacterial proteins. Infect Disord Drug Targets 6, 85-106

147. Stachyra, T., Dini, C., Ferrari, P., Bouhss, A., van Heijenoort, J., MenginLecreulx, D., Blanot, D., Biton, J., and Le Beller, D. (2004) Fluorescence detection-based functional assay for high-throughput screening for MraY. Antimicrob Agents Chemother 48, 897-902

148. Weppner, W. A., and Neuhaus, F. C. (1977) Fluorescent substrate for nascent peptidoglycan synthesis. Uridine diphosphate-N-acetylmuramyl-( $\mathrm{N}^{\varepsilon}-5$ dimethylaminonaphthalene-1-sulfonyl)penta peptide. J Biol Chem 252, 22962303

149. Geis, A., and Plapp, R. (1978) Phospho-N-acetylmuramoyl-pentapeptidetransferase of Escherichia coli K12. Properties of the membrane-bound and the extracted and partially purified enzyme. Biochim Biophys Acta 527, 414-424

150. Branstrom, A. A., Midha, S., Longley, C. B., Han, K., Baizman, E. R., and Axelrod, H. R. (2000) Assay for identification of inhibitors for bacterial MraY translocase or MurG transferase. Anal Biochem 280, 315-319

151. Hyland, S. A., and Anderson, M. S. (2003) A high-throughput solid-phase extraction assay capable of measuring diverse polyprenyl phosphate: sugar-1phosphate transferases as exemplified by the WecA, MraY, and MurG protein. Anal Biochem. 317, 156-165

152. Solapure, S. M., Raphael, P., Gayathri, C. N., Barde, S. P., Chandrakala, B., Das, K. S., and De Sousa, S. M. (2005) Development of a microplate-based scintillation proximity assay for MraY using a modified substrate. $J$ Biomol Screen. 10, 149-156

153. Ravishankar, S., Kumar, V. P., Chandrakala, B., Jha, R. K., Solapure, S. M., and de Sousa, S. M. (2005) Scintillation proximity assay for inhibitors of Escherichia coli MurG and, optionally, MraY. Antimicrob Agents Chemother 49, 1410-1418

154. Shapiro, A. B., Jahic, H., Gao, N., Hajec, L., and Rivin, O. (2012) A highthroughput, homogeneous, fluorescence resonance energy transfer-based assay for phospho-N-acetylmuramoyl-pentapeptide translocase (MraY). J Biomol Screen 17, 662-672 
155. Li, K., and Kurosu, M. (2008) Synthetic studies on Mycobacterium tuberculosis specific fluorescent park's nucleotide probe. Heterocycles 76, 455-469

156. Mitachi, K., Mohan, P., Siricilla, S., and Kurosu, M. (2014) One-pot protectionglycosylation reactions for synthesis of lipid II analogues. Chemistry 20, 45544558

157. Kurosu, M., and Li, K. (2009) Synthetic studies towards the identification of novel capuramycin analogs with antimycobacterial activity. Heterocycles. 2008; 76:455-469 77, 217-225

158. Raymond, J. B., Mahapatra, S., Crick, D. C., and Pavelka, M. S. (2005) Identification of the namH gene, encoding the hydroxylase responsible for the $\mathrm{N}$ glycolylation of the mycobacterial peptidoglycan. J Biol Chem 280, 326-333

159. Chen, K. T., Kuan, Y. C., Fu, W. C., Liang, P. H., Cheng, T. J., Wong, C. H., and Cheng, W. C. (2013) Rapid preparation of mycobacterium N-glycolyl lipid I and lipid II derivatives: a biocatalytic approach. Chemistry 19, 834-838

160. Wolfe, L. M., Mahaffey, S. B., Kruh, N. A., and Dobos, K. M. (2010) Proteomic definition of the cell wall of Mycobacterium tuberculosis. J Proteome Res $\mathbf{9}$, 5816-5826

161. Mahapatra, S., Crick, D. C., and Brennan, P. J. (2000) Comparison of the UDP-Nacetylmuramate:L-alanine ligase enzymes from Mycobacterium tuberculosis and Mycobacterium leprae. J Bacteriol 182, 6827-6830

162. Brandish, P. E., Burnham, M. K., Lonsdale, J. T., Southgate, R., Inukai, M., and Bugg, T. D. (1996) Slow binding inhibition of phospho-N-acetylmuramylpentapeptide-translocase (Escherichia coli) by mureidomycin A.J Biol Chem 271, 7609-7614

163. Nuhaus, F. C. (1971) Initial translocation reaction in biosynthesis of peptidoglycan by bacterial membranes. Acc. Chem. Res. 4, 297-303

164. Wang, Y., Siricilla, S., Aleiwi, B. A., and Kurosu, M. (2013) Improved synthesis of capuramycin and its analogues. Chemistry 19, 13847-13858

165. Kurosu, M., Li, K., and Crick, D. C. (2009) Concise synthesis of capuramycin. Org Lett 11, 2393-2396

166. Aleiwi, B. A., Schneider, C. M., and Kurosu, M. (2012) Synthesis of ureidomuraymycidine derivatives for structure activity relationship studies of muraymycins. J. Org. Chem. 77, 3859-3867 
167. Boojamra, C. G., Lemoine, R. C., Blais, J., Vernier, N. G., Stein, K. A., Magon, A., Chamberland, S., Hecker, S. J., and Lee, V. J. (2003) Synthetic dihydropacidamycin antibiotics: a modified spectrum of activity for the pacidamycin class. Bioorg Med Chem Lett 13, 3305-3309

168. Lin, Y. I., Li, Z., Francisco, G. D., McDonald, L. A., Davis, R. A., Singh, G., Yang, Y., and Mansour, T. S. (2002) Muraymycins, novel peptidoglycan biosynthesis inhibitors: semisynthesis and SAR of their derivatives. Bioorg Med Chem Lett. 12, 2341-2344

169. Zhang, J. H., Chung, T. D., and Oldenburg, K. R. (1999) A simple statistical parameter for use in evaluation and validation of high throughput screening assays. J Biomol Screen. 4, 67-73

170. Al-Dabbagh, B., Mengin-Lecreulx, D., and Bouhss, A. (2008) Purification and characterization of the bacterial UDP-GlcNAc:undecaprenyl-phosphate GlcNAc1-phosphate transferase WecA. J Bacteriol 190, 7141-7146

171. Barr, K., and Rick, P. D. (1987) Biosynthesis of enterobacterial common antigen in Escherichia coli. In vitro synthesis of lipid-linked intermediates. J Biol Chem 262, 7142-7150

172. Alexander, D. C., and Valvano, M. A. (1994) Role of the rfe gene in the biosynthesis of the Escherichia coli O7-specific lipopolysaccharide and other Ospecific polysaccharides containing N-acetylglucosamine. J Bacteriol 176, 70797084

173. Pasquina, L. W., Santa maria, J. P. S., and Walker, S. (2013) Teichoic acid biosynthesis as an antibiotic target. Curr. Opin. Microbiol. 16, 531-537

174. Ha, S., Chang, E., Lo, M. C., Men, H., Park, P., Ge, M., and Walker, S. (1999) The kinetic characterization of Escherichia coli MurG using synthetic substrate analogues. J. Am. Chem. Soc. 121, 8415-8426

175. Al-Dabbagh, B., Blanot, D., Mengin-Lecreulx, D., and Bouhss, A. (2009) Preparative enzymatic synthesis of polyprenyl-pyrophosphoryl-Nacetylglucosamine, an essential lipid intermediate for the biosynthesis of various bacterial cell envelope polymers. Anal. Biochem. 391, 163-165

176. Amer, A. O., and Valvano, M. A. (2001) Conserved amino acid residues found in a predicted cytosolic domain of the lipopolysaccharide biosynthetic protein WecA are implicated in the recognition of UDP-N-acetylglucosamine. Microbiology $147,3015-3025$ 
177. Anderson, M. S., Eveland, S. S., and Price, N. P. (2000) Conserved cytoplasmic motifs that distinguish sub-groups of the polyprenol phosphate:Nacetylhexosamine-1-phosphate transferase family. FEMS Microbiol. Lett. 191, $169-175$

178. Merino, S., Jimenez, N., Molero, R., Bouamama, L., Regue, M., and Tomas, J. M. (2011) A UDP-HexNAc:polyprenol-P GalNAc-1-P transferase (WecP) representing a new subgroup of the enzyme family. J Bacteriol 193, 1943-1952

179. Patel, K. B., Toh, E., Fernandez, X. B., Hanuszkiewicz, A., Hardy, G. G., Brun, Y. V., Bernards, M. A., and Valvano, M. A. (2012) Functional characterization of UDP-glucose:undecaprenyl-phosphate glucose-1-phosphate transferases of Escherichia coli and Caulobacter crescentus. J Bacteriol 194, 2646-2657

180. Lehrman, M. A. (1994) A family of UDP-GlcNAc/MurNAc: polyisoprenol-P GlcNAc/MurNAc-1-P transferases. Glycobiology 4, 768-771

181. Rush, J. S., Rick, P. D., and Waechter, C. J. (1997) Polyisoprenyl phosphate specificity of UDP-GlcNAc:undecaprenyl phosphate N-acetylglucosaminyl 1-P transferase from E.coli. Glycobiology 7, 315-322

182. Al-Dabbagh, B., Olatunji, S., Crouvoisier, M., El Ghachi, M., Blanot, D., Mengin-Lecreulx, D., and Bouhss, A. (2016) Catalytic mechanism of MraY and WecA, two paralogues of the polyprenyl-phosphate N-acetylhexosamine 1phosphate transferase superfamily. Biochimie 127, 249-257

183. Mitachi, K., Siricilla, S., Klaic, L., Clemons, W. M., Jr., and Kurosu, M. (2015) Chemoenzymatic syntheses of water-soluble lipid I fluorescent probes. Tetrahedron Lett 56, 3441-3446

184. Nikonenko, B. V., Reddy, V. M., Protopopova, M., Bogatcheva, E., Einck, L., and Nacy, C. A. (2009) Activity of SQ641, a capuramycin analog, in a murine model of tuberculosis. Antimicrob Agents Chemother 53, 3138-3139

185. Dubuisson, T., Bogatcheva, E., Krishnan, M. Y., Collins, M. T., Einck, L., Nacy, C. A., and Reddy, V. M. (2010) In vitro antimicrobial activities of capuramycin analogues against non-tuberculous mycobacteria. J Antimicrob Chemother 65, 2590-2597

186. Wayne, L. G., and Sramek, H. A. (1979) Antigenic differences between extracts of actively replicating and synchronized resting cells of Mycobacterium tuberculosis. Infect Immun. 24, 363-370

187. Gengenbacher, M., Rao, S. P., Pethe, K., and Dick, T. (2010) Nutrient-starved, non-replicating Mycobacterium tuberculosis requires respiration, ATP synthase 
and isocitrate lyase for maintenance of ATP homeostasis and viability. Microbiology 156, 81-87

188. Reddy, V. M., Einck, L., and Nacy, C. A. (2008) In vitro antimycobacterial activities of capuramycin analogues. Antimicrob Agents Chemother 52, 719-721

189. Hsieh, M. H., Yu, C. M., Yu, V. L., and Chow, J. W. (1993) Synergy assessed by checkerboard. A critical analysis. Diagn Microbiol Infect Dis 16, 343-349

190. Meletiadis, J., Pournaras, S., Roilides, E., and Walsh, T. J. (2010) Defining fractional inhibitory concentration index cutoffs for additive interactions based on self-drug additive combinations, Monte Carlo simulation analysis, and in vitro-in vivo correlation data for antifungal drug combinations against Aspergillus fumigatus. Antimicrob Agents Chemother 54, 602-609

191. Griffin, J. E., Gawronski, J. D., Dejesus, M. A., Ioerger, T. R., Akerley, B. J., and Sassetti, C. M. (2011) High-resolution phenotypic profiling defines genes essential for mycobacterial growth and cholesterol catabolism. PLoS Pathog 7, e1002251

192. Birch, A. J., Holzapfel, C. W., Richards, R. W. (1963) Diterpenoid nature of pleuromutilin. Chem. Ind. 14, 374-375

193. Stipkovits, L., Ripley, P. H., Tenk, M., Glavits, R., Molnar, T., and Fodor, L. (2005) The efficacy of valnemulin (Econor) in the control of disease caused by experimental infection of calves with Mycoplasma bovis. Res Vet Sci 78, 207-215

194. Jones, R. N., Fritsche, T. R., Sader, H. S., and Ross, J. E. (2006) Activity of retapamulin (SB-275833), a novel pleuromutilin, against selected resistant grampositive cocci. Antimicrob Agents Chemother 50, 2583-2586

195. Novak, R., and Shlaes, D. M. (2010) The pleuromutilin antibiotics: a new class for human use. Curr. Opini. Invest. Drugs 11, 182-911

196. Sader, H. S., Biedenbach, D. J., Paukner, S., Ivezic-Schoenfeld, Z., and Jones, R. N. (2012) Antimicrobial activity of the investigational pleuromutilin compound BC-3781 tested against Gram-positive organisms commonly associated with acute bacterial skin and skin structure infections. Antimicrob Agents Chemother 56, $1619-1623$

197. Paukner, S., Sader, H. S., Ivezic-Schoenfeld, Z., and Jones, R. N. (2013) Antimicrobial activity of the pleuromutilin antibiotic BC-3781 against bacterial pathogens isolated in the SENTRY antimicrobial surveillance program in 2010. Antimicrob Agents Chemother 57, 4489-4495 
198. Long, K. S., Poehlsgaard, L. S., Hansen, L.H., Hobbie, S. N., Böttger, E. C., and Vester, B. (2009) Single 23S rRNA mutations at the ribosomal peptidyl transferase centre confer resistance to valnemulin and other antibiotics in Mycobacterium smegmatis by perturbation of the drug binding pocket. Molecular Microbiology 71, 1218-1222

199. Dong, Y. J., Meng, Z. H., Mi, Y. Q., Zhang, C., Cui, Z. H., Wang, P., and Xu, Z. B. (2015) Synthesis of novel pleuromutilin derivatives. Part 1: preliminary studies of antituberculosis activity. Bioorg Med Chem Lett 25, 1799-1803

200. Lotesta, S. D., Liu, J., Yates, E. V., Krieger, I., Sacchettini, J. C., Freundlich, J. S., and Sorensen, E. J. (2011) Expanding the pleuromutilin class of antibiotics by de novo chemical synthesis. Chem Sci 2, 1258-1261

201. Ascher, G., Stauffer, F., Berner, H., and Mang, R. (2003) Tuberculosis treatment using pleuromutilin derivatives. Google Patents

202. Wang, X., Ling, Y., Wang, H., Yu, J., Tang, J., Zheng, H., Zhao, X., Wang, D., Chen, G., Qui, W., and Tao, J. (2012) Novel Pleuromutilin derivatives as antibacterial agents: Synthesis, biological evaluation and molecular docking studies. Bioorg. Med. Chem. Lett. 22, 6166-6172

203. Shang, R., Pu, X., Xu, X., Xin, Z., Zhang, C., Guo, W., Liu, Y., and Liang, J. (2014) Synthesis and biological activities of novel pleuromutilin derivatives with a substituted thiadiazole moiety as potent drug-resistant bacteria inhibitors. $J$. Med. Chem. 57, 5664-5678

204. Wang, Q., Wang, Y., and Kurosu, M. (2012) A new oxyma derivative for nonracemizable amide-forming reactions in water. Org Lett 14, 3372-3375

205. Wang, Y., Aleiwi, B. A., Wang, Q., and Kurosu, M. (2012) Selective esterifications of primary alcohols in a water-containing solvent. Org Lett 14, 4910-4913

206. Aleiwi, B. A., Mitachi, K., and Kurosu, M. (2013) Mild and convenient Nformylation protocol in water-containing solvents. Tetrahedron Lett 54, 20772081

207. Siricilla, S., Mitachi, K., Yang, J., Eslamimehr, S., Lemieux, M. R., Meibohm, B., Ji, Y., and Kurosu, M. A New combination of a pleuromutilin derivative and doxycycline for treatment of MDR Acinetobacter baumannii. In Press

208. Wang, Z., Hop, C. E., Leung, K. H., and Pang, J. (2000) Determination of in vitro permeability of drug candidates through a caco-2 cell monolayer by liquid chromatography/tandem mass spectrometry. J Mass Spectrom 35, 71-76 
209. Bøsling, J., Poulsen, S. M., Vester, B., and Long, K. S. . (2003) Resistance to the peptidyl transferase inhibitor tiamulin caused by mutation of ribosomal protein L3. Antimicrob. Agents Chemother. 47, 2892-2896

210. Pringle, M., Poehlsgaard, J., Vester, B., and Long, K. S. (2004) Mutations in ribosomal protein $\mathrm{L} 3$ and $23 \mathrm{~S}$ ribosomal RNA at the peptidyl transferase center are associated with reduced susceptibility to tiamulin in Brachyspira spp. isolates. Mol. Microbiol. 54, 1295-1306

211. Gentry, D. R., Rittenhouse, S. F., McCloskey, L., and Holmes, D. J. (2007) Stepwise exposure of Staphylococcus aureus to pleuromutilins is associated with stepwise acquisition of mutations in $\mathrm{rplC}$ and minimally affects susceptibility to retapamulin Antimicrob. Agents and Chemother. 51, 2048-2052

212. Long, K. S., Hansen, L. H., Jakobsen, L., and Vester, B. (2006) Interaction of pleuromutilin derivatives with the ribosomal peptidyl transferase center. Antimicrob Agents Chemother 50, 1458-1462

213. Long, K. S., Poehlsgaard, J., Kehrenberg, C., Schwarz, S., and Vester, B. (2006) The Cfr rRNA methyltransferase confers resistance to Phenicols, Lincosamides, Oxazolidinones, Pleuromutilins, and Streptogramin A antibiotics. Antimicrob Agents Chemother 50, 2500-2505

214. Peleg, A. Y., de Breij, A., Adams, M. D., Cerqueira, G. M., Mocali, S., Galardini, M., Nibbering, P. H., Earl, A. M., Ward, D. V., Paterson, D. L., Seifert, H., and Dijkshoorn, L. (2012) The success of acinetobacter species; genetic, metabolic and virulence attributes. PLoS One 7, e46984

215. Liu, Y., Zhang, J., and Ji, Y. (2016) PCR-based approaches for the detection of clinical methicillin-resistant Staphylococcus aureus. Open Microbiol J 10, 45-56

216. Snewin, V. A., Gares, M. P., Gaora, P. O., Hasan, Z., Brown, I. N., and Young, D. B. (1999) Assessment of immunity to mycobacterial infection with luciferase reporter constructs. Infect Immun 67, 4586-4593

217. Betts, J. C., Lukey, P. T., Robb, L. C., McAdam, R. A., and Duncan, K. (2002) Evaluation of a nutrient starvation model of Mycobacterium tuberculosis persistence by gene and protein expression profiling. Mol Microbiol 43, 717-731

218. Futatsugi, L., Saito, H., Kakegawa, T., and Kobayashi, H. (1997) Growth of an Escherichia coli mutant deficient in respiration. FEMS Microbiol Lett 156, 141145

219. Bagramyan, K., Mnatsakanyan, N., Poladian, A., Vassilian, A., and Trchounian, A. (2002) The roles of hydrogenases 3 and 4 , and the $\mathrm{F}_{0} \mathrm{~F}_{1}$-ATPase, in $\mathrm{H}_{2}$ production by Escherichia coli at alkaline and acidic pH. FEBS Lett 516, 172-178 
220. Rezwan, M., Laneelle, M. A., Sander, P., and Daffe, M. (2007) Breaking down the wall: fractionation of mycobacteria. J Microbiol Methods 68, 32-39

221. Bouhss, A., Crouvoisier, M., Blanot, D., and Mengin-Lecreulx, D. (2004) Purification and characterization of the bacterial MraY translocase catalyzing the first membrane step of peptidoglycan biosynthesis. J Biol Chem 279, 2997429980

222. Ma, Y., Munch, D., Schneider, T., Sahl, H. G., Bouhss, A., Ghoshdastider, U., Wang, J., Dotsch, V., Wang, X., and Bernhard, F. (2011) Preparative scale cellfree production and quality optimization of MraY homologues in different expression modes. $J$ Biol Chem 286, 38844-38853

223. Chojnacki, T., Jankowski, W., Mankowski, T., and Sasak, W. (1975) Preparative separation of naturally occurring mixtures of polyprenols on hydroxyalkoxypropyl-Sephadex. Anal Biochem 69, 114-119

224. Skorupińska-Tudek, K., Bienkowski, T., Olszowska, O., Furmanowa, M., Chojnacki, T., Danikiewicz, W., and E. Swiezewska. (2003) Divergent pattern of polyisoprenoid alcohols in the tissues of Coluria geoides: a new electrospray lonization MS approach. Lipids 38, 981-990

225. Danilov, L. L., Druzhinina, T. N., Kalinchuk, N. A., Maltsev, S. D., and Shibaev, V. N. (1989) Polyprenyl phosphates: synthesis and structure-activity relationship for a biosynthetic system of Salmonella anatum O-specific polysaccharide. Chem Phys Lipids 51, 191-203

226. Ohrt, C., Willingmyre, G. D., Lee, P., Knirsch, C., and Milhous, W. (2002) Assessment of azithromycin in combination with other antimalarial drugs against Plasmodium falciparum in vitro. Antimicrob Agents Chemother 46, 2518-2524

227. Kong, Y., Yao, H., Ren, H., Subbian, S., Cirillo, S. L., Sacchettini, J. C., Rao, J., and Cirillo, J. D. (2010) Imaging tuberculosis with endogenous beta-lactamase reporter enzyme fluorescence in live mice. Proc Natl Acad Sci U S A 107, 1223912244

228. McGinnity, D. F., Soars, M. G., Urbanowicz, R. A., and Riley, R. J. (2004) Evaluation of fresh and cryopreserved hepatocytes as in vitro drug metabolism tools for the prediction of metabolic clearance. Drug Metab Dispos 32, 1247-1253 


\section{VITA}

Shajila Siricilla was born in 1981 in Kerala, India. She completed her Bachelor's degree (BPharm) in Pharmacy in 2003 from JSS College of Pharmacy, Dr. MGR Medical University of Chennai, India. In 2007, She earned her Post Graduate Diploma (Master's degree) in Business Administration from the Symbiosis Center of Distance Learning, Pune, India. From 2004 to 2008 she worked for Juggat Pharmaceuticals, India (QC Chemist), Kaya Skin Clinic, India (Client Cordinator) and Knight Frank, India (Management Cordinator). In August 2011, she joined Pharmaceutical Sciences PhD Program at University of Tennessee Health Science Center, Memphis under the supervision of Dr. Michio Kurosu.

She has been elected for membership of the American Association of Pharmaceutical Scientists (AAPS), the American Chemical Society (ACS) and the American Association for the Advancement of Science (AAAS). During her graduate school, she obtained a patent related to her dissertation research, published ten peerreviewed articles in scientific journals and was invited to share her scientific findings at many scientific conferences in the United States. Shajila was a recipient of AAPS Drug Discovery and Development Interface Travel Award (2016) and UTHSC CGHS John Autian Student Enrichment Travel Award (2016). During her time at UTHSC she enjoyed her research assistant and teaching assistant jobs for the College of Pharmacy at UTHSC. She has mentored many PharmD, summer students and junior PhD students on research projects. During her $\mathrm{PhD}$, she studied development of new tuberculosis drug leads that are effective in killing dormant Mycobacterium tuberculosis and development of novel in vitro assays for screening and validation of selective inhibitors of molecular targets. 